\title{
SIGNAL QUALITY ENHANCEMENT OF ESOPHAGEAL PRESSURE SIGNALS IN MECHANICALLY VENTILATED PATIENTS
}

by

Michael Zara

B.Eng Biomedical Engineering, Ryerson University, 2016

\author{
A thesis \\ presented to Ryerson University \\ in partial fulfillment of the \\ requirements for the degree of \\ Master of Applied Science \\ in the program of \\ Biomedical Engineering
}

Toronto, Ontario, Canada, 2019

CMichael Zara, 2019 


\section{AUTHOR'S DECLARATION FOR ELECTRONIC SUBMISSION OF A THESIS}

I hereby declare that I am the sole author of this thesis. This is a true copy of the thesis, including any required final revisions, as accepted by my examiners.

I authorize Ryerson University to lend this thesis to other institutions or individuals for the purpose of scholarly research.

I further authorize Ryerson University to reproduce this thesis by photocopying or by other means, in total or in part, at the request of other institutions or individuals for the purpose of scholarly research.

I understand that my dissertation may be made electronically available to the public. 
Signal Quality Enhancement of Esophageal Pressure Signals in Mechanically Ventilated Patients Master of Applied Science 2019

Michael Zara

Biomedical Engineering

Ryerson University

\begin{abstract}
The Esophageal Pressure (Peso) signal can be used to monitor the respiratory mechanics of critically ill patients in the Intensive Care Unit (ICU), and has been successfully used in guiding mechanical ventilation strategies to improve patient outcomes. However, cardiogenic oscillations (CGOs) are a major source of interference, which not only makes it challenging in interpreting the patient's respiratory mechanics, but can also cause false triggers in the mechanical ventilator resulting in a patient-ventilator asynchrony.

In this thesis, we present a Peso enhancement scheme using Ensemble Empirical Mode Decomposition (EEMD) to suppress CGO interference. The proposed method was applied to synthetically generated Peso signals as well as real-world Peso signals from mechanically ventilated ICU patients. The proposed technique has been shown to significantly reduce the amplitude fluctuations caused by CGOs. The technique's performance has been assessed through Face Validation by our collaborating clinicians, and is found to be suitable in not only suppressing CGO, but also extracting CGO from clinically acquired Peso signals.
\end{abstract}




\section{Acknowledgements}

First and foremost, I would like to thank my supervisor, Dr. Sri Krishnan, for his continued support and guidance over the years. It has been a long road with many twists and turns, but your role as my GPS (Graduate Program Supervisor) has finally brought us to the end of this journey. I appreciate all that you have done for me - from opening up opportunities and connecting me with different organizations/people, to the pep-talks that made me feel as though I could accomplish everything I could ever dream of. Thank you very much for this wild ride; I have done a lot of growing not only as an aspiring researcher, but as a person as well under your wing. I will definitely look back fondly on my years as your student.

To our clinical collaborators - Dr. Laurent Brochard, Dr. Irene Telias, Dr. Lu Chen, and Dr. Remi Coudroy - thank you very much for taking time out of your busy schedules to collaborate on the work that ultimately resulted in this thesis. Your ability to communicate the clinical and physiological significance of these signals made this research all the more enjoyable to pursue.

To my friends from the Signal Analysis Research (SAR) Lab - Sammy Abdou, Yash Athavale, Dharmendra Gurve, Syem Ishaque, Binh Nguyen, Michael Nigro, Jeevan Pant, Fayez Qureshi, and Garima Sharma - thank you very much for such a supportive atmosphere that made it enjoyable to work in EPH439. I am sure we have spent many hours chatting (and not getting research work done), but I will truly miss hearing all your stories, experiences, and thoughts. To my friends from abroad who only spent a few months with us - Mariana Cardoso, Denis Delisle, Alan Floriano, Hamilton Rivera, Flavia Loterio, Mario Jimenez, Nicolas Valencia, Yves Luduvico, Pedro Cunha, Deepak Prasad, and Swostik Acharya - I certainly haven't forgotten about all of you and the experiences we've shared! 
To Sourav Mukhopadhyay, I want to thank you for your guidance throughout this research work. I truly enjoyed working alongside you; I learned a lot from you this past year. You are also a fantastic teacher, and I hope to one day develop the ability to explain concepts the way you do.

To Alice Rueda, you are a fantastic buddy. I loved all the time spent in the lab doing work and chatting about "nothing." Despite spending a lot of our time being unproductive, and instead opting to chat and eat, those are the moments that I will remember and treasure. I've learned so much from you and have grown to become a better researcher and person because of those lessons. Thank you.

To Sharadha Kolappan, Matthew Hotradat, and Nhu Nguyen - we did it!! I'm glad that we went through this journey together. It was always comforting to see your faces on the rare occasions we bumped into each other. We can finally treat ourselves to ice cream (and all sorts of junk food)!

To Fionn Ly, you've watched me grow over the years and I want you to know that you played a massive role in that. You have been a constant source of support, and for that I want to thank you from the bottom of my heart.

Last but certainly not least, I want to thank my parents, Miriam and Ruben Zara, as well as my sister, Rochelle Zara, for their love as well as their support of my education. I love you. 


\section{Dedication}

To my friends, family, colleagues, mentors, teachers, students, and acquaintances - I've spent a

lot of time growing over the last few year-all because of you. Each of you have impacted my life, and I have learned something new from every single one of you. You have acted as a catalyst for my growth, and I am much happier because of it.

Thank you. 


\section{Contents}

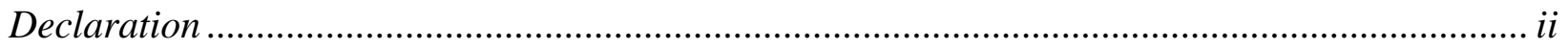

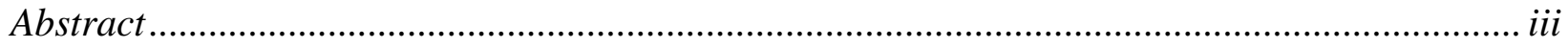

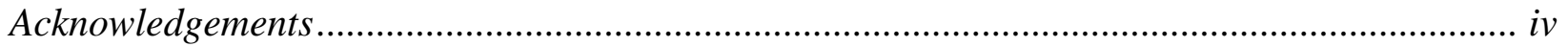

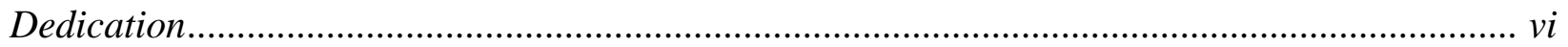

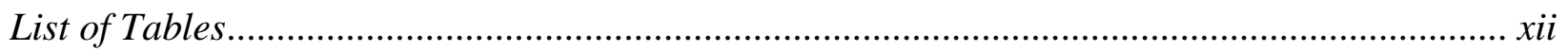

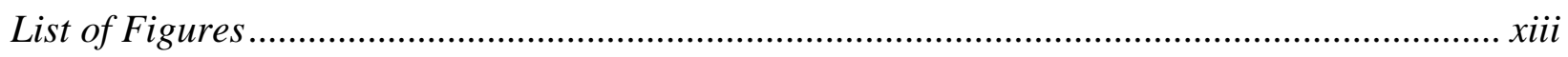

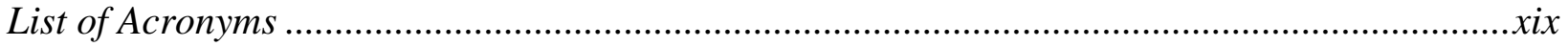

\section{Chapter 1 Introduction}

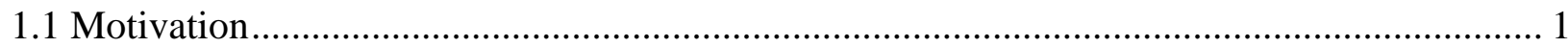

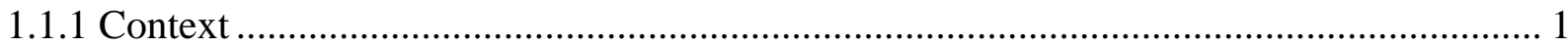

ICU Treatments: Mechanical Ventilation.................................................................... 2

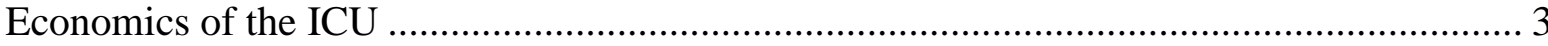

1.1.2 Towards the future of Mechanical Ventilation ............................................................. 5

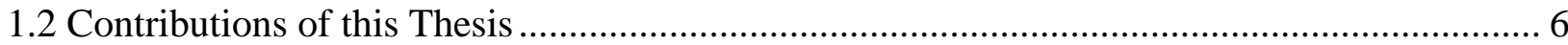

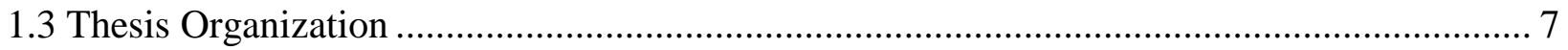

\section{Chapter 2 Background}

2.1 The Respiratory System \& Physiology of Breathing ...................................................... 9

2.1.1 Anatomical Structures Involved in Breathing ...................................................... 11

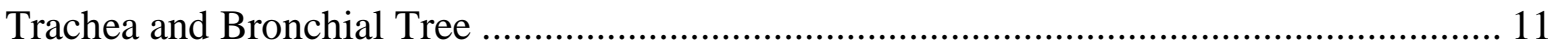

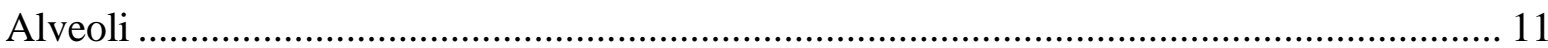

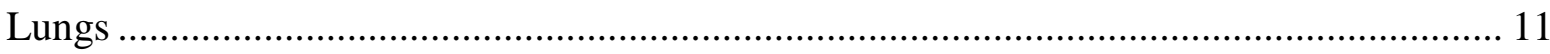

Thoracic Cavity and Muscles of Respiration .............................................................. 12

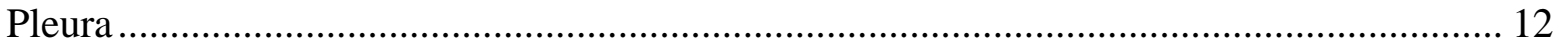

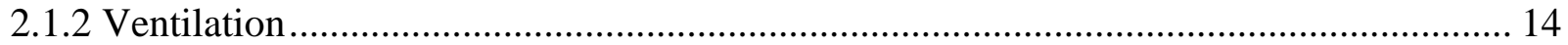

Relationship between Pressure, Airflow, and Volume.................................................. 14

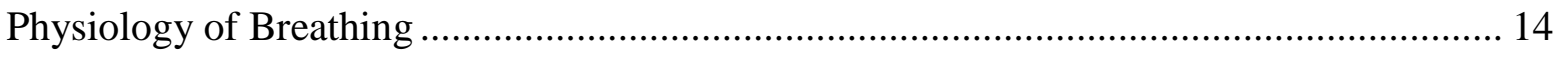

Factors Affecting Alveolar Volume .................................................................. 15 
2.2 Respiratory Monitoring \& Guiding Mechanical Ventilation................................................. 15

2.2.1 Respiratory Monitoring in Mechanical Ventilation ...................................................... 16

Significance of Transpulmonary Pressure in Guiding Mechanical Ventilation ..................... 17

Esophageal Pressure as Surrogate for Pleural Pressure .................................................... 18

Significance of Esophageal Pressure in Guiding Ventilation............................................... 18

Acquisition of Esophageal Pressure ............................................................................ 19

2.3 Characteristics of the Esophageal Pressure (Peso) Signal ...................................................... 21

2.3.1 Time-Domain Characteristics of Peso.......................................................................... 21

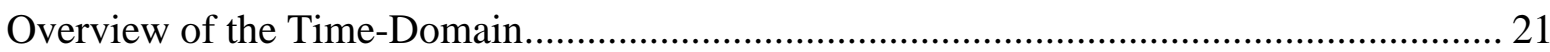

Time-Domain Characteristics of the Raw Peso Signal ...................................................... 22

2.3.2 Frequency-Domain Characteristics of Peso ……………........................................... 29

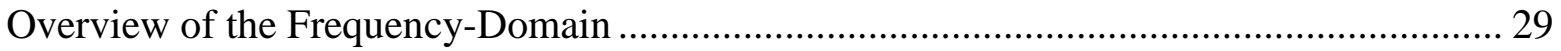

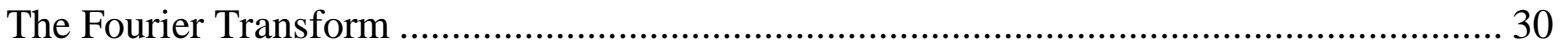

Frequency Characteristics of the Raw Peso Signal ............................................................. 31

2.3.3 Sources of Interference in the Peso Signal................................................................. 32

Sources of Interference in the Measured Peso Signal ......................................................... 32

2.3.4 Relevant work related to CGO Suppression in Peso Signals ........................................... 34

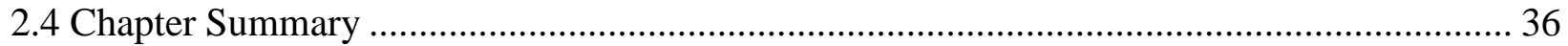

Chapter 3 The Proposed Enhancement Scheme: Characterizing the Peso and CGO Signals, Preliminary Experimentation \& Proof-of-Concept

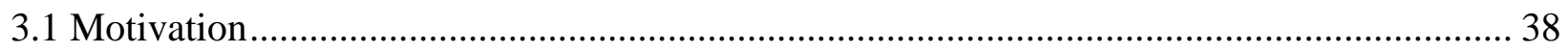

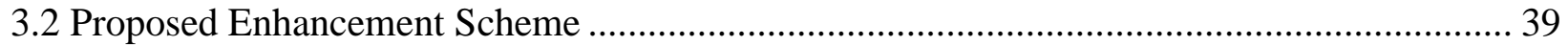

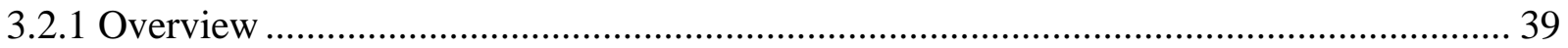

Empirical Mode Decomposition (EMD) …………….................................................. 39

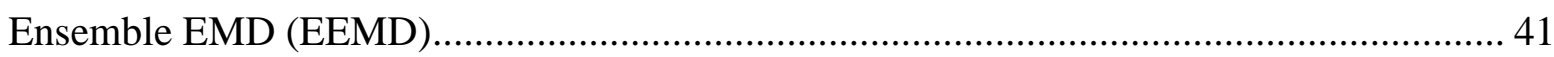

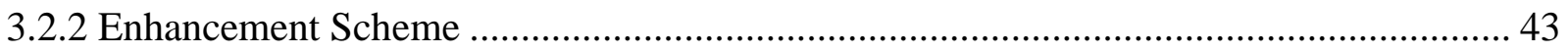

3.3 Characterizing the CGO Interference and Proof-of-Concept of Proposed Enhancement Scheme 46

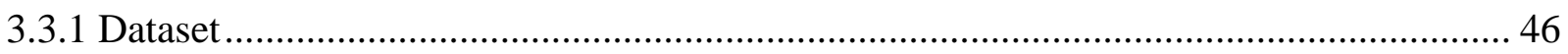

3.3.2 Experimental Setup and Validation - Characterizing CGOs \& Proof-of-Concept Results 47

3.3.3 Results and Discussions 48 


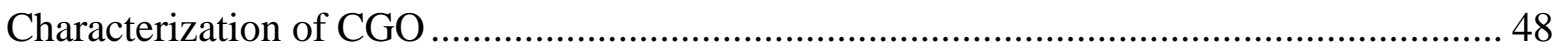

Application of Proposed Enhancement Scheme - A Proof-of-Concept................................. 50

3.4 Refining the Proposed Enhancement Scheme ...................................................................... 57

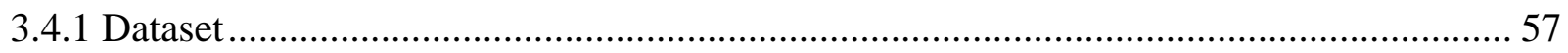

3.4.2 Experimental Setup - Refining the Proposed Enhancement Scheme ……………......... 57

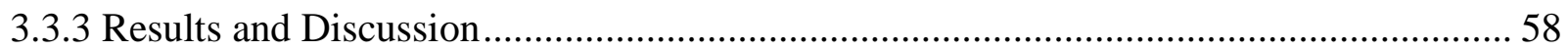

3.5 Application of the (Refined) Proposed Enhancement Scheme to Spontaneously Breathing Patients - Another Proof-of-Concept......................................................................................... 63

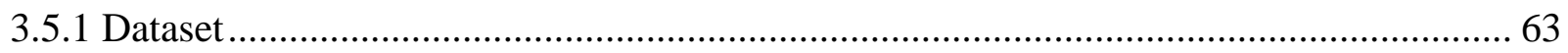

3.5.2 Experimental Setup and Validation - Proof-of-Concept Results for Spontaneously

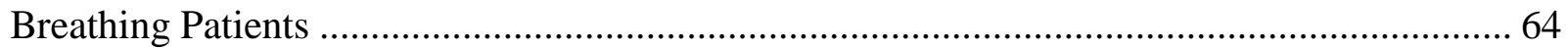

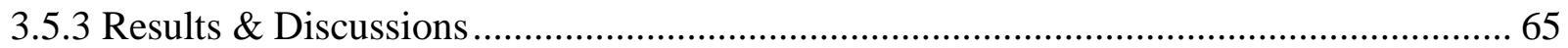

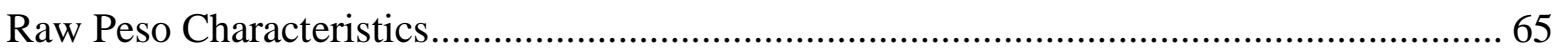

Application of the (Refined) Proposed Enhancement Scheme - A Proof-of-Concept ......... 66

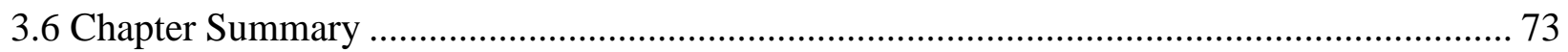

\section{Chapter 4 FrankEinstein's Peso Signals}

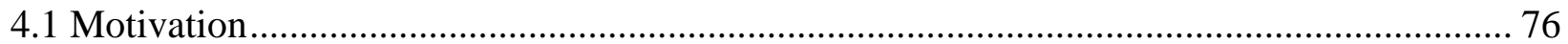

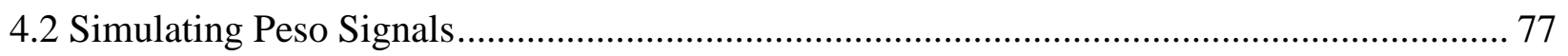

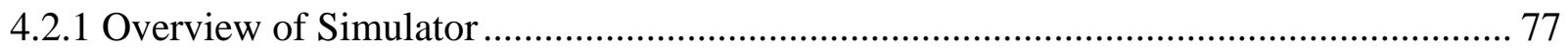

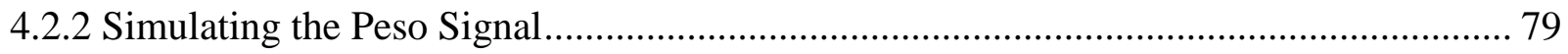

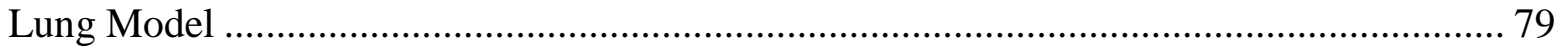

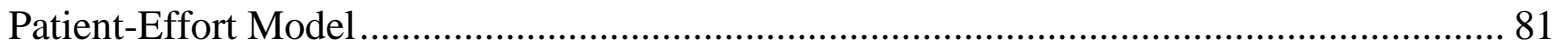

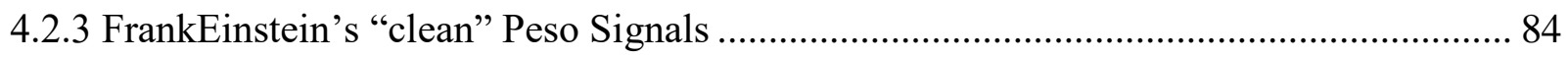

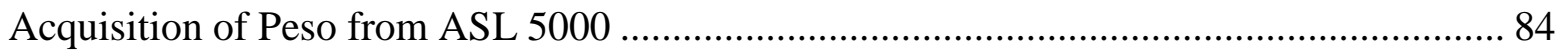

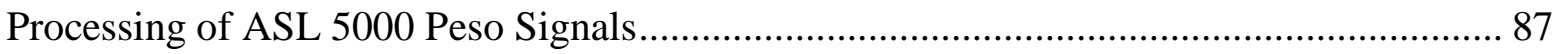

4.3 Extracting CGO Interference .................................................................................... 90

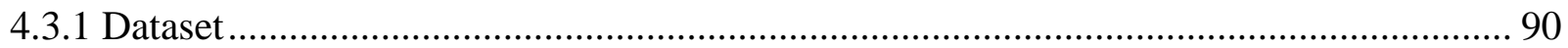

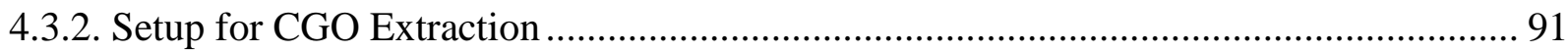

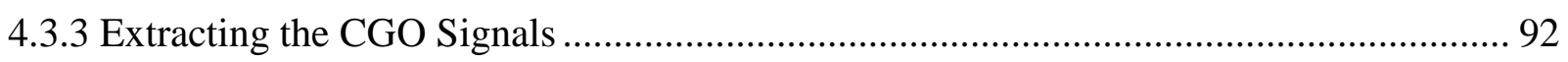

4.4 “Reanimating” FrankEinstein’s "Noisy” Peso Signal .............................................................. 96

4.5 Applying the Proposed Enhancement Scheme to FrankEinstein's "Noisy" Peso Signal....... 99

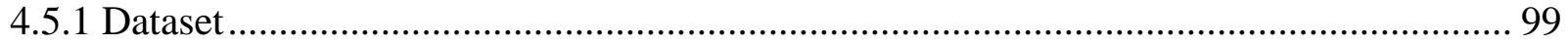


4.5.2 Experimental Setup …………………………….............................................. 100

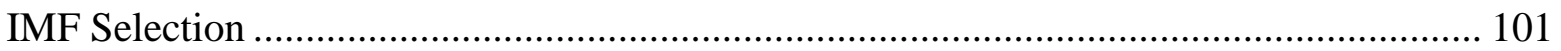

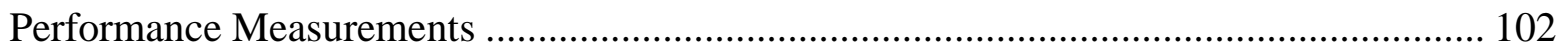

Testing the Performance of the Proposed Enhancement Scheme …….............................. 104

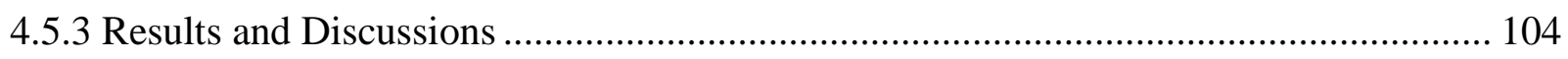

Effects of SIR on CGO Suppression and PRD using the Proposed Enhancement Scheme 104

Relationship between SIR and PRD .............................................................................. 109

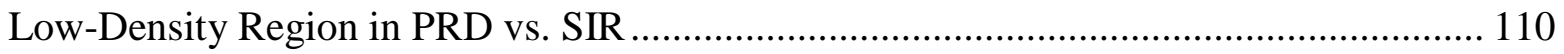

Operating Limits of the Proposed Enhancement Scheme ………………........................... 111

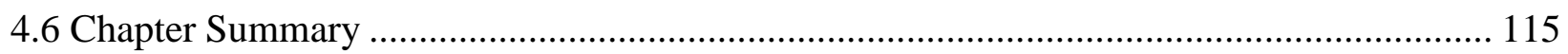

\section{Chapter 5 CGO Suppression in Real ICU Patients}

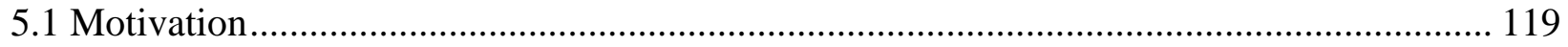

5.2 Application of the Proposed Enhancement Scheme on Real ICU Patients .......................... 119

5.2.1 Dataset................................................................................................................. 119

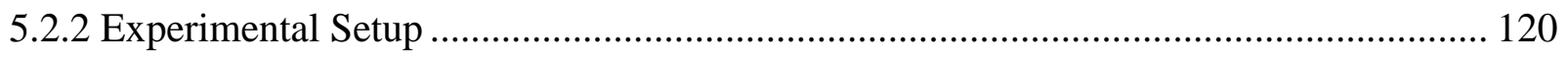

Method for Suppressing CGO in Peso ..................................................................... 120

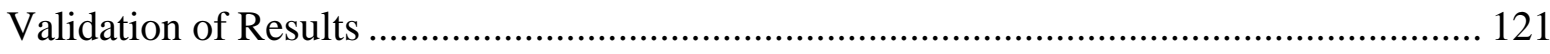

5.2.3 Results and Discussions ....................................................................................... 121

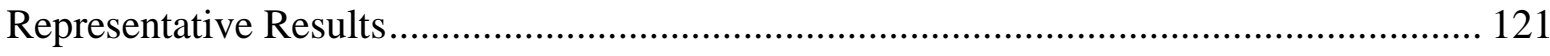

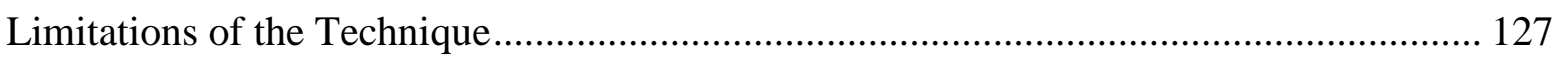

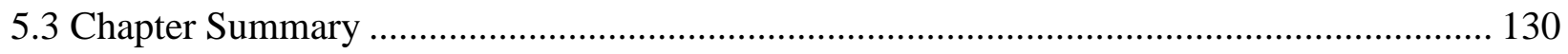

\section{Chapter 6 Conclusions and Future Work}

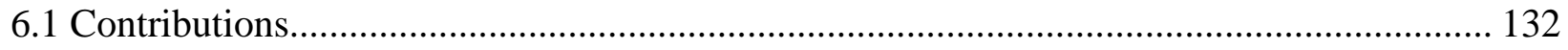

6.1.1 Characterization of Peso and CGO ...................................................................... 133

6.1.2 Objective Performance Measures........................................................................... 133

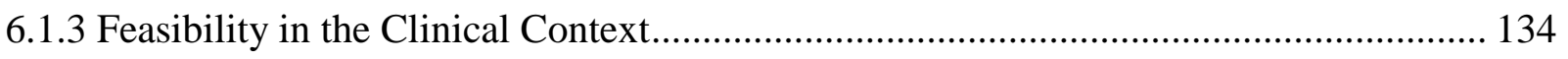

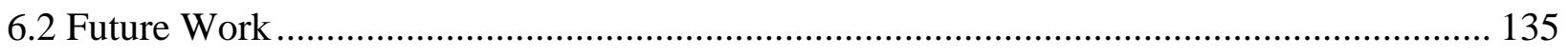

Overcoming Technical Limitations …………………………………………………..... 135

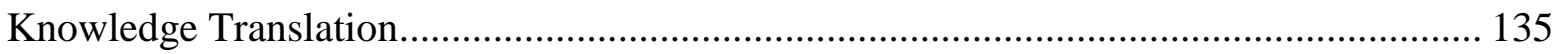

Adapting to other Mechanical Ventilation and Respiratory Monitoring Applications ....... 135 


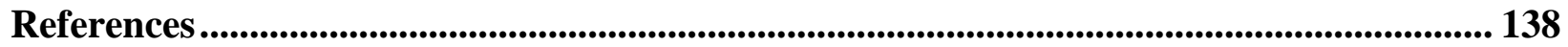




\section{List of Tables}

Table 1: Summary of the dataset used for getting a baseline understanding of CGOs. .... 47

Table 2: Summary of the dataset used for refining the proposed enhancement scheme... 57

Table 3: The IMFs used for CGO extraction and Peso enhancement for passively breathing

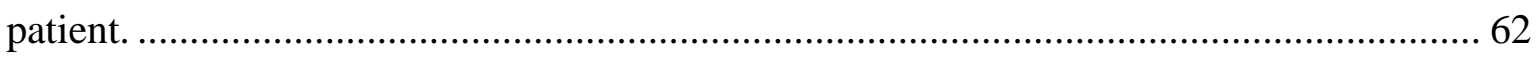

Table 4: Summary of the dataset used for refining the proposed enhancement scheme... 64

Table 5: Patient-Effort Model parameters using sinusoidal breath profile for FrankEinstein's

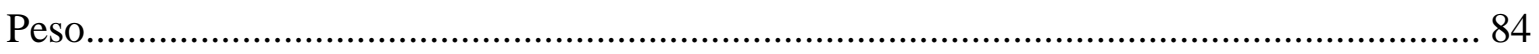

Table 6: Parameters used to simulate "clean" Peso on the ASL 5000 .......................... 85

Table 7: Summary of the dataset used for extracting CGOs for FrankEinstein's Peso Signal.

Table 8: A summary of FrankEinstein's "Noisy” Peso signals dataset............................ 97

Table 9: A summary of FrankEinstein's dataset. ..................................................... 100

Table 10: Descriptive statistic measures of PRD for each of the normalized CGO amplitude

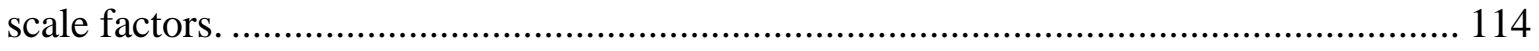

Table 11: Summary of the dataset used for testing the final proposed enhancement scheme. 


\section{List of Figures}

1.1 Top 10 medical conditions among adult ICU patients in Canada during 2007-2008 and 2013-2014... 2

1.2 A breakdown of Ontario's projected expenditure for the 2019-2020 year .................. 4

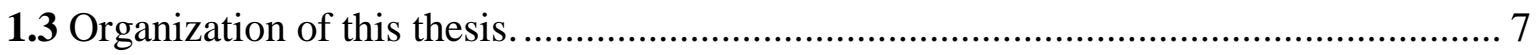

2.1 The anatomical structures of the respiratory system, which includes the Conducting Zone (nose to bronchial tree) and the Respiratory Zone (lungs and alveoli). The Diaphragm lies

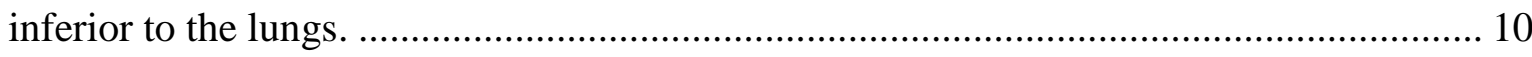

2.2 The anatomical structures of the chest wall and muscles of respiration, and a visual representation of the pleural membranes and the pleural cavity ................................... 13

2.3 Clinical setup used to acquire data from mechanically ventilated patients................ 20

2.4 Nasal insertion of esophageal catheter (left) and the corresponding Peso signal (right)

2.5 The time-domain representation of the Flow signal (top) and Esophageal Pressure/Peso signal (bottom). The horizontal dashed red line in the Flow signal indicates the threshold between mechanical insufflation and mechanical exsufflation. Clinical parameters in the Peso signal are illustrated in the figure above. In the breath designated by (1), the vertical red dashed lines indicate the total inspiration time $\left(\mathrm{t}_{\text {inspiration }}\right)$ and the green vertical dashed line represents total expiration time ( $\left.\mathrm{t}_{\text {expiration}}\right)$. In (2), the blue horizontal dotted lines and arrow show the Peso Swing $(\Delta \mathrm{P})$. In (3), the esophageal pressure-time product (PTPes) is highlighted in orange. The dark blue curve represents the chest wall pressure during a relaxed state $\left(\mathrm{P}_{\mathrm{cw}, \mathrm{rel}}\right)$, which is obtained during passive breathing. The small pressure fluctuations caused by the beating heart known as cardiogenic oscillations (CGOs), which are not labelled, are present throughout the Peso signal. 23

2.6 A Peso signal from a passively breathing patient. The red vertical dashed lines represent the duration of one inspiratory cycle. At the onset of inspiration, the mechanical ventilator provides positive pressure to allow airflow into the lungs resulting in the positive deflection

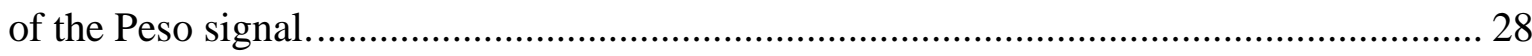


2.7 A Peso signal with significant CGO interference. A period of one CGO cycle is highlighted in red.

2.8 A Peso signal with an esophageal spasm occurring between 75-80 seconds. The spasm is caused by a swallowing ..... 33

3.1 A flowchart representing how EEMD generates the IMFs of a given signal.... 42

3.2. The workflow of the proposed enhancement scheme. The raw ("noisy") signal will first be decomposed using a decomposition algorithm (i.e. EMD or EEMD) to generate the IMFs. The IMFs relevant to the physiological process being measured are then selected. The IMFs are summed together to reconstruct a "clean" signal that is free from noisy/interference seen in the original raw signal.

3.3. The complete methodology of this research work. This is a collaborative project between Ryerson University and St. Michael's Hospital under the Institute of Biomedical Engineering, Science, and Technology (iBEST). The raw Peso signal is acquired using an esophageal catheter by clinicians at St. Michael's Hospital, as shown in the blue box. The raw Peso signal is then provided to the Signal Analysis Research (SAR) Lab at Ryerson University, where the proposed enhancement technique (shown in the pink box) is used to remove/suppress the CGO interference from the Peso signal. 45 3.4. The Peso signal in time-domain on the left. The low-flow inflation maneuver manifests itself as a linear increase in pressure during inspiration and a steep decline in pressure during expiration, while the CGOs are represented by small oscillatory fluctuations throughout the time-series. The magnitude spectrum of the Peso signal is shown on the right. The fundamental frequency of the CGO is indicated by the blue arrow, and the harmonics of the CGO are labelled with green arrows.

3.5. The IMFs of the Peso signal using EMD. 51

3.6. (A) Comparison between the original Peso (black), the reconstructed CGO using IMFs 1 to 2 (red), and the reconstructed CGO using IMFs 1 to 3 (blue). The DC value of the original Peso was removed, an artificial value of $-3 \mathrm{cmH}_{2} \mathrm{O}$ was added to the $\mathrm{CGO}$ using IMFs 1 to 2 (red), and a value of $-5 \mathrm{cmH}_{2} \mathrm{O}$ was added to the CGO using IMFs 1 to 3 (blue) for a clear comparison of the waveforms. (B) A portion of the original Peso signal containing only CGO. The section of the signal portrayed in this plot is encapsulated by the yellow box in (A). (C) A portion of the original Peso signal containing the low-flow maneuver, which 
is extracted from the green box in (A). The expiration process of the maneuver is shown in the purple box 53

3.7. (A) The reconstructed Peso signal using IMFs 3 to 9 shown in red. The blue arrow indicates that a CGO cycle still persists after the application of the proposed signal enhancement scheme. (B) The reconstructed Peso signal using IMFs 4 to 9 shown in blue.

(C) The reconstructed Peso signal using IMFs 5 to 9 shown in green. 55

3.8. Mode-mixing problem observed in IMFs 1 and 2. The oscillatory components in IMF 1 that are shown in red should have appeared in IMF2. 56

3.9. The IMFs generated using EMD for the passively breathing patient (recreation of Figure 3.5). 59

3.10. The IMFs generated using EEMD for the same passively breathing patient seen in Figure 3.9. 60

3.11. (A) The original raw Peso (black) with the DC value removed, the extracted CGOs using and EEMD's IMF 2 to IMF 3 (red) with an added value of $-3 \mathrm{cmH}_{2} \mathrm{O}$, and EMD's IMF 1 to IMF 3 (blue) with an added $-5 \mathrm{cmH}_{2} \mathrm{O}$. (B) The enhanced Peso using EEMD's IMF 4 to IMF 9 (red) and EMD's IMF 4 to IMF 9 (blue) superimposed on the original raw Peso (grey).

3.12. The time-domain representation of a Peso from a spontaneously breathing patient (left) and the magnitude spectrum (right). The peaks corresponding to CGO and its harmonics are indicated by red arrows. The red dotted vertical line marks the threshold between Peso activity, which are represented by peaks to the left of the threshold, and the CGO activity. The magnitude spectrum has been zoomed in to get a clear representation of the CGO and its harmonics 66

3.13. The IMFs using EEMD of the Peso for a spontaneously breathing patient 67

3.14. A comparison of the raw Peso (black) and the reconstructed Peso using the following IMF combinations: (A) IMFs 4 to 11 (red) (B) IMFs 5 to 11 (blue) (C) IMFs 6 to 11 (green) 71

3.15. (A) The time-domain representations of the Peso signals (left) and the corresponding magnitude spectra (right). The raw Peso is shown in black while the reconstructed Peso using IMFs 4 to 11 are plotted in red. The peak indicated by the green arrow shows that the activity from the CGO has not been suppressed. (B) Similarly, the time-domain (left) and frequency- 
domain (right) representations of the Peso signals are shown. The raw Peso is plotted in black and the reconstructed Peso using IMFs 5 to 11 are shown in blue. The green arrow in (B) points to the CGO peak described previously in (A). The reconstructed Peso (blue) has significantly reduced the magnitude of the peak 72

3.16. The block diagram of the refined enhancement scheme used to suppress CGO. The EEMD algorithm is chosen to decompose the raw Peso signal into its IMFs. Through mode selection, it is possible to reconstruct both the CGO and the Peso separately . 75

4.1. The IngMar Medical ASL 5000 (Active Servo Lung) ........................................... 78

4.2. A functional overview of the ASL 5000 78

4.3. The single compartment model of the lungs used in generating FrankEinstein's Peso signals (left) and the equivalent analog circuit equivalent (right). The single compartment model consists of a one linear or parabolic resistor $(\mathrm{R})$, and one linear compliance $(\mathrm{C}) \ldots 80$ 4.4. An illustration of eight of the nine parameters used to define the $\mathrm{P}_{\text {mus }} /$ Peso trace (red) for the sinusoidal breath model for one full breath cycle. Not pictured in this figure is the breathing rate. 83

4.5. An illustration of the FrankEinstein's Peso trace (red) using the sinusoidal breath model for one full breath cycle. Note that the illustration is not to scale. 84 4.6. The "clean" Peso signal simulated from the ASL 5000. The max pressure amplitude of $\Delta \mathrm{P}$ is $-10 \mathrm{cmH}_{2} \mathrm{O}$. The blue arrows indicate a sharp inflection at the transition between i) the end of expiratory hold and the beginning of inspiratory increase, and ii) the end of expiration release and the beginning of expiration hold.... 86 4.7. The "clean" Peso signal simulated from the ASL 5000 to illustrate Peso Signal \#9 from Table 6. The max pressure amplitude of $\Delta \mathrm{P}$ was varied between -5 to $-15 \mathrm{cmH}_{2} \mathrm{O}$......... 86 4.8. The workflow used to generate the final "clean" Peso for FrankEinstein's Peso signal

4.9. The workflow used to extract the CGO interference from 20 randomly selected patients in Dataset \#3. 92

4.10. The first eight IMFs of a passively breathing patient's Peso. The IMFs related to CGO are indicated by a red box. 93

4.11. The time-domain representation of the Raw Peso (black) and the reconstructed CGO using IMFs 4 to 5 (red) is shown on the right. ... 95 
4.12. The normalized CGO that was extracted from a passively breathing patient 95

4.13. The process of creating FrankEinstein's "noisy" Peso signal 96

4.14. A set of FrankEinstein Peso signals using one CGO that was scaled 10 different times 98

4.15. An example of "noisy" FrankEinstein Peso signals with different SIR levels. On the left is a Peso with SIR of $22.8671 \mathrm{~dB}$ and the Peso on the right has an SIR of $2.8671 \mathrm{db}$. The CGO interference is common between both signals albeit with different scaling factors. The CGO for the left Peso has a scale factor of 0.1 and the CGO for the right Peso has a scale factor of 1 105

4.16. Three signals are shown in each plot: i) the reference Peso in black, which was obtained from the ASL 5000, ii) the FrankEinstein "noisy" Peso shown is in green, and iii) the reconstructed Peso resulting from the proposed enhancement scheme is in red. (A) The SIR of the FrankEinstein Peso is $22.8617 \mathrm{~dB}$. The reconstructed Peso has a PRD of 4.3\%. (B) The SIR is $2.8617 \mathrm{~dB}$, and the reconstructed Peso's PRD is $11.2 \%$ 107

4.17. The reference Peso (black), the FrankEinstein "noisy" Peso using a CGO scaling factor of $1 \mathrm{cmH}_{2} \mathrm{O}$ (green), and the reconstructed Peso (red). It is evident that the reconstructed Peso is unable to preserve the reference Peso's morphology. 108

4.18. A scatterplot of PRD (\%) with respect to SIR (dB) for all 1800 "noisy" FrankEinstein Peso signals. A first-order polynomial model was fit to the data and is plotted in red. A region containing a low density of datapoints above PRD $=\sim 40 \%$ is outlined in a green circle. The datapoints represent significant outliers, most of which are associated with one specific CGO

4.19. A boxplot showcasing the distribution of PRD (\%) for each of the $10 \mathrm{CGO}$ scale factors 114

5.1. A Peso signal obtained from a spontaneously breathing patient receiving PSV. This Peso signal is representative of a typical breathing pattern and morphology. 122

5.2. A Peso signal obtained from a spontaneously breathing patient receiving PSV. This Peso signal is representative of a similar breath profile/morphology shown in Figure 5.1, but with a different CGO morphology.

5.3. A Peso signal obtained from a spontaneously breathing patient receiving PSV. This Peso signal is representative of another commonly observed breath profile/morphology....... 124 
5.4. A Peso signal obtained from a passively breathing patient receiving mechanical ventilation support. 124

5.5. A Peso signal obtained from a passively breathing patient. An occlusion occurs at $t=$ 41 seconds to $t=46$ seconds. 126 5.6. A Peso signal obtained from a spontaneously breathing patient using a t-piece. An occlusion occurs from $\mathrm{t}=154$ seconds to $\mathrm{t}=172$ seconds 126

5.7. A Peso signal obtained from a spontaneously breathing patient receiving PSV. The large transients represent esophageal spasms caused by the patient swallowing their saliva. The segment of the signal highlighted in the green box is used in Figure 5.9 to illustrate the effects of the esophageal spasm on signal reconstruction using the proposed enhancement scheme 127

5.8. The Peso segment highlighted in green from Figure 5.8. The raw Peso is shown in black in both figures. (A) The reconstructed Peso signal is shown in red. The EEMD algorithm was applied to the raw signal with the esophageal spasms still in tact. (B) The reconstructed Peso signal shown in blue. The esophageal spasm from the raw signal was clipped manually before applying the EEMD algorithm

5.9. The relation between the amount of time required to decompose a Peso signal using EEMD with respect to the duration of the Peso signal... 130

6.1. Experimental setup and data acquisition of EAdi signals 137 


\section{List of Acronyms}

ARDS Acute Respiratory Distress Syndrome

CGO Cardiogenic Oscillation

CMV Conventional Mechanical Ventilation

COPD Chronic Obstructive Pulmonary Disorder

ECG Electrocardiogram

EEG Electroencephalogram

EMD Empirical Mode Decomposition

EEMD Ensemble Empirical Mode Decomposition

EMG Electromyogram

FT Fourier Transform

ICU Intensive Care Unit

IMF Intrinsic Mode Function

NMBA Neuromuscular Blocking Agent

Peso Esophageal Pressure

Palv Alveolar Pressure

$\mathbf{P}_{\text {aw }}$ Airway Pressure

$\mathbf{P}_{\mathbf{B}}$ Barometric Pressure 
$\mathbf{P}_{\mathbf{c w}, \text { rel }}$ Pressure of the Chest-wall under a relaxed condition

PL Transpulmonary Pressure

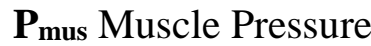

$\mathbf{P}_{\mathbf{p l}}$ Pleural Pressure

$\Delta \mathbf{P}$ Peso Swing (amplitude of Peso)

PRD Percent Root-Mean Square Difference

PSV Pressure Support Ventilation

PTPes Pressure-Time Product of Esophageal Pressure

SIR Signal-to-Interference Ratio

VILI Ventilator-Induced Lung Injury 


\section{Chapter 1}

\section{Introduction}

\subsection{Motivation}

\subsubsection{Context}

With the hustle and bustle of daily life, we often take our bodies for granted. Many of us will occasionally feel aches and pains, and perhaps even experience sniffles in the nose and tickles in the throat. It would not be a rare thought to brush these off as minor annoyances that we simply have to cope with. However, worries start to set in when these minor annoyances escalate and become a hindrance. Instances like these warrant a trip to the doctor to ask the quintessential question, "What is wrong with me?" It is easy to forget that our bodies consist of several different organ systems working together to ensure that proper physiological processes are maintained. Oftentimes, the visit to the doctor's office will reveal that the organ systems are working relatively well, and the problems that prompted the visit in the first place can be resolved quite simply. Unfortunately, there are times when a person's organ systems start to fail as a result of a severe or life-threatening illness or injury. In cases like these, the person enters the health care system through the Intensive Care Unit (ICU), where they are monitored closely by a team of highly trained critical care specialists in order to maintain normal bodily functions.

In the Canadian context, $11 \%$ of adult hospital stays around the country, excluding Quebec, required time in the ICU [1]. The top 10 medical conditions among Canadian ICU patients, most of which are related to the cardiovascular and respiratory systems, are shown in Figure 1.1. Many 
people associate these conditions with mortality - and rightfully so, because some of the leading causes of death in Canada are in fact related to diseases affecting the cardiovascular and respiratory systems [2].

\begin{tabular}{|c|c|c|c|c|}
\hline & Top medical conditions & $\begin{array}{l}\text { Volume } \\
\text { (percentage of } \\
\text { all medical } \\
\text { ICU patients) } \\
\text { 2007-2008 }\end{array}$ & $\begin{array}{l}\text { Volume } \\
\text { (percentage of } \\
\text { all medical } \\
\text { ICU patients) } \\
\text { 2013-2014 }\end{array}$ & $\begin{array}{l}\text { Change }(p<0.05) \text { in } \\
\text { proportion, 2007- } \\
2008 \text { to } 2013-2014\end{array}$ \\
\hline 1 & $\begin{array}{l}\text { Myocardial infarction/ } \\
\text { shock/arrest }\end{array}$ & $19,773(19 \%)$ & $14,911(13 \%)$ & \\
\hline 2 & Arrhythmia & $7,412(7 \%)$ & $6,874(6 \%)$ & \\
\hline 3 & Heart failure & $5,853(6 \%)$ & $5,821(5 \%)$ & \\
\hline 4 & $\begin{array}{l}\text { Chronic obstructive pulmonary } \\
\text { disease (COPD) }\end{array}$ & $5,051(5 \%)$ & $5,691(5 \%)$ & \\
\hline 5 & Other/unspecified sepsis & $3,044(3 \%)$ & $5,137(5 \%)$ & \\
\hline 6 & Poisoning/toxic effect of drug & $3,947(4 \%)$ & $4,263(4 \%)$ & \\
\hline 7 & Respiratory failure & $2,378(2 \%)$ & $4,046(4 \%)$ & \\
\hline 8 & $\begin{array}{l}\text { Unstable angina/arteriosclerotic } \\
\text { heart disease (ASHD) }\end{array}$ & $6,816(6 \%)$ & $3,685(3 \%)$ & \\
\hline 9 & $\begin{array}{l}\text { Other/miscellaneous } \\
\text { cardiac disorder }\end{array}$ & $2,691(3 \%)$ & $3,389(3 \%)$ & \\
\hline 10 & Diabetes & $2,413(2 \%)$ & $2,962(3 \%)$ & \\
\hline
\end{tabular}

Figure 1.1. Top 10 medical conditions among adult ICU patients in Canada during 2007-2008 and 2013-2014. Source: [1].

\section{ICU Treatments: Mechanical Ventilation}

In Canadian ICUs, 1 in 3 patients are prescribed a life-supporting treatment known as mechanical ventilation [1]. The aim of mechanical ventilation is to: get oxygen $\left(\mathrm{O}_{2}\right)$ into the patient's lungs, remove carbon dioxide $\left(\mathrm{CO}_{2}\right)$, reduce the amount of effort required to breathe, and perform the function of breathing for patients who have lost all ability to breathe due to injury or medication [3]. From Figure 1.1, the volume of ICU patients suffering from Respiratory Failure 
has increased from $2 \%$ to $4 \%$ over a span of six years, which suggests that mechanical ventilation will continue to play a significant role in treating ICU patients.

Mechanical ventilation in its most basic form is known as Conventional Mechanical Ventilation (CMV). CMV aims to improve gas exchange and ease respiratory distress by delivering a positive pressure to the patient's airways using a pre-set tidal volume at a fixed respiratory rate [4]. While CMV has been used to save lives and improve the quality of care in critically ill patients, it has also been associated with significant drawbacks. Because CMV delivers a fixed volume at a fixed rate, it does not consider the patient's respiratory drive. If a mismatch between the patient's respiratory drive and the operation of the mechanical ventilator occurs, a phenomenon known as Asynchrony is observed [4]. Poor patient-ventilator interactions (i.e. asynchrony) can result in the deterioration of gas exchange, discomfort, cardiovascular impairment, and even increased mortality rates, thereby putting the patient at greater risk [5]-[6]. To avoid the potential risks of asynchrony, the patient's respiratory drives are suppressed using sedatives. Unfortunately, the use of paralysis-inducing drugs on the respiratory muscles opens up a new set of challenges including muscle atrophy of the respiratory muscles (e.g. diaphragm), adverse drug-related effects, and excessive ventilation, and Ventilator-Induced Lung Injuries (VILI) [4]-[7]. In both cases, CMV has the potential to inflict harm as well as make it more difficult to wean patients off the mechanical ventilator, resulting in longer ICU stays.

\section{Economics of the ICU}

The ICU is an integral part of the Canadian health care system, but it comes with a hefty cost. On average, the cost to run an ICU is three times more than a general hospital ward due to high staff-to-patient ratios and the need for specialized equipment for complex treatments [1], [8]. Despite only a fraction of patients requiring ICU interventions, the ICU's resource-intensive nature 
takes up a substantial amount of a hospital's resources [1]. The economic aspect of running an ICU is important to consider, because of the Canadian health care system model; our health care system is publicly funded, which gives Canadian residents reasonable access to necessary medical health care services without having to pay directly out-of-pocket [9]. Each of the Provincial Governments in Canada are responsible for the funding of their respective provincial health care systems. Figure 1.2 breaks down the projected expenditure for the Ontario Government during the 2019-2020 year. The pie-chart indicates that $38.9 \%$ of Ontario's funding is provided to the health sector, which is by far the greatest resource allocation [10]. It is expected that the needs for ICU beds and other hospital resources are to increase significantly in the future due to the aging population and the increasing severity of illnesses [1], [8]. As a result, more burden will be placed on an alreadyailing health care system, and provincial resources will need to be reallocated to account for changes in the population's health.

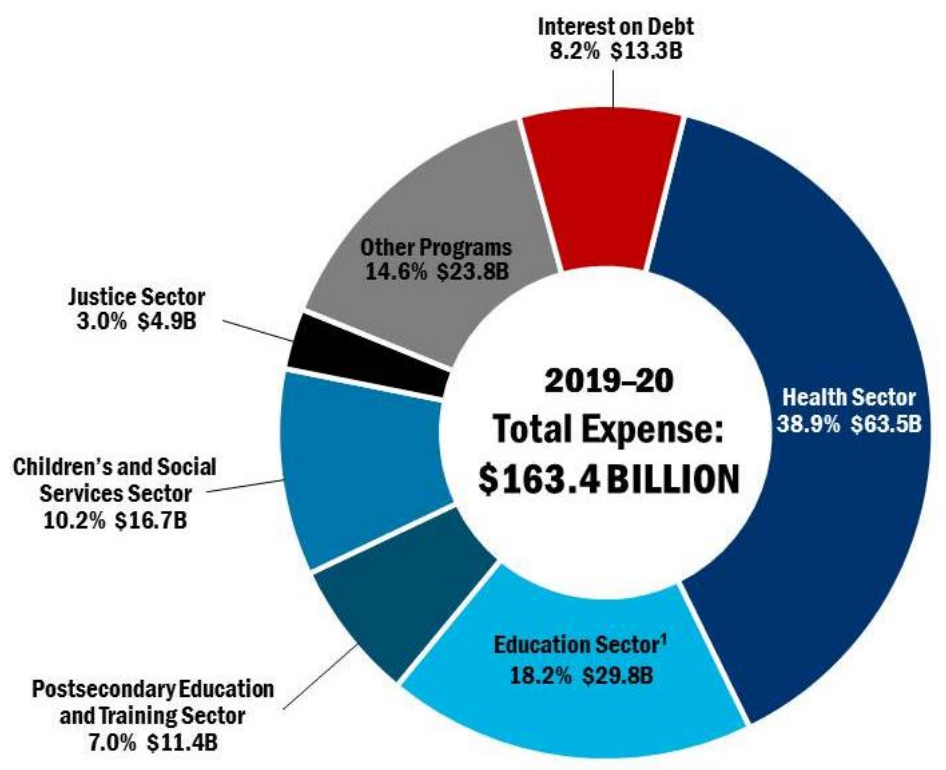

1 Excludes Teachers' Pension Plan. Teachers' Pension Plan expense is included in Other Programs. Note: Numbers may not add due to rounding.

Figure 1.2. A breakdown of Ontario's projected expenditure for the 2019-2020 year. Source: [10] 


\subsubsection{Towards the future of Mechanical Ventilation}

It is clear that CMV has a huge cost not only in terms of financial burdens on the government funding agencies, but also in terms of the quality of care for ICU patients. From the perspective of the health care actors involved in treating ICU patients, new modes of mechanical ventilation are being developed to overcome the issues associated with CMV. There has been a shift in paradigm towards having patients take on more of the ventilatory effort; the mechanical ventilator should be assisting the patient instead of taking over the breathing process entirely. Through assisted modes of mechanical ventilation, the patient's respiratory drive is able to trigger the operation of the mechanical ventilator [5]. In spite of new developments, issues such as asynchrony and VILI still persists as major problems for mechanical ventilation management [6][7], [11]-[12].

Given the current problems still associated with mechanical ventilation, new "lungprotective" mechanical ventilation strategies are being developed. The goal of these strategies is not only aimed at improving oxygenation and reducing the work of breathing, but also protecting the lungs from further injuries in an effort to decrease mortality rates and shorten ICU stays [13]. Measurements and derived parameters from Esophageal Pressure (Peso) and Transpulmonary Pressure $\left(\mathrm{P}_{\mathrm{L}}\right)$ have sparked interest in recent years towards guiding mechanical ventilation treatments, and have proven to be helpful in monitoring a patient's respiratory mechanics [14]. While there is potential for Peso, clinicians run into problems involving cardiogenic oscillation (CGO) interference caused by the beating heart, which makes interpreting the Peso signal difficult, and can even result in asynchrony through ventilator auto-triggering [15]-[17]. Other technical limitations associated with Peso prevent it from being widely used in clinical practice. As a result, there is ongoing research towards validating the use of Peso and $\mathrm{P}_{\mathrm{L}}$ in the clinical context [18]. 


\subsection{Contributions of this Thesis}

The aim of this thesis is to develop a signal quality enhancement scheme to suppress the CGO contamination from the Peso signal to help clinicians extract meaningful insights into the physiology of their mechanically ventilated patients.

The first contribution presented in this thesis is a proposed enhancement scheme for CGO suppression in Peso signals using adaptive, data-driven time-series decomposition techniques, namely Empirical Mode Decomposition (EMD) and its variant, Ensemble Empirical Mode Decomposition (EEMD). To our knowledge, this is the first time that the EMD and EEMD algorithms have been applied to enhance the signal quality of the Peso signal. Additionally, while the main aim of the proposed enhancement scheme is to suppress CGO, it can also be used to extract the CGO. The ability to extract CGO independently may be useful in gaining an understanding of the patient's heart physiology.

The second contribution of this thesis is the creation of synthetic "noisy" Peso signals, which have been termed, "FrankEinstein's Peso signals." The "noisy" Peso signals were created by superimposing CGO signals that were extracted from real ICU patients onto simulated Peso signals from the IngMar Medical ASL 5000 breathing simulator. The FrankEinstein Peso signals provide a means of objectively quantifying the performance of the proposed enhancement scheme. Furthermore, the level of CGO interference present in the signal can be adjusted, which is helpful in determining the operating limits of the proposed technique.

The final contribution of this thesis is the application of the proposed enhancement scheme, which was refined through the previous two contributions, to real ICU patients. Results of the enhanced Peso signals are shown, and technical limitations of the technique are described. 


\subsection{Thesis Organization}

The organization of this thesis is shown in Figure 1.3.

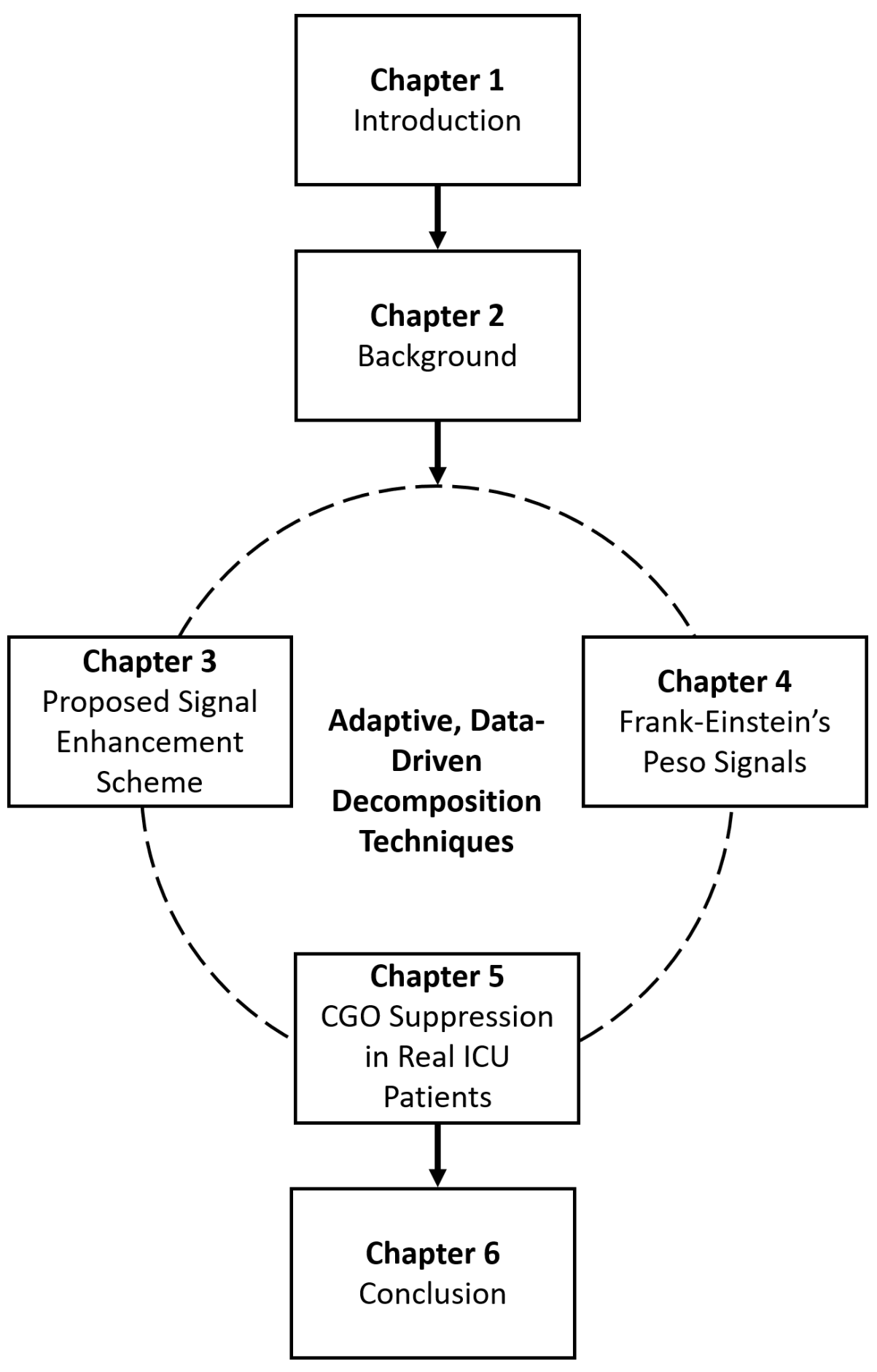

Figure 1.3. Organization of this thesis. 
The remainder of this thesis is organized as follows:

Chapter 2 - Background, provides a description of the anatomy of the respiratory system and the physiology of breathing. The significance of $\mathrm{P}_{\mathrm{L}}$ and its relation to the Peso signal in managing mechanical ventilation will be discussed. Time-domain and frequency-domain characteristics of the Peso signal and the CGO interference will be explained from a biomedical signal processing perspective.

Chapter 3 - The Proposed Enhancement Scheme: Characterizing the Peso and CGO Signals, Preliminary Experimentation \& Proof-of-Concept, introduces the proposed enhancement scheme for the purposes of suppressing CGO from Peso signals. Several experiments are carried out in this chapter to characterize the Peso and CGO signals to develop and refine the enhancement technique.

Chapter 4 - FrankEinstein's Peso Signals, describes how the FrankEinstein Peso dataset was created. The proposed enhancement scheme was applied to varying levels of CGO to determine the relationship between Signal-to-Interference Ratio (SIR) and an error measurement known as Percent Root Mean Square Difference (PRD). Furthermore, a suggested operating limit for the enhancement technique is proposed.

Chapter 5 - CGO Suppression in Real ICU Patients, applies the proposed enhancement scheme to real ICU patients. Representative results are shown, and limitations of the technique are described.

Finally, Chapter 6 - Conclusions and Future Works, presents the concluding remarks about the proposed technique, and future directions such as the potential to adapt the proposed technique for other signal processing applications in the ICU. 


\section{Chapter 2}

\section{Background}

\subsection{The Respiratory System \& Physiology of Breathing}

Mechanical ventilation primarily acts on a patient's respiratory system to improve pulmonary gas exchange and relieve respiratory distress in patients. The main role of the respiratory system is to help in breathing, which is otherwise known as respiration. Respiration includes processes such as ventilation, which describes the movement of air into and out of the lungs, and gas exchange, which ensures that oxygen $\left(\mathrm{O}_{2}\right)$ is transported to our tissues and carbon dioxide $\left(\mathrm{CO}_{2}\right)$ is removed from the body [19]. From a functional perspective, the respiratory system is divided into two regions: i) the Conducting Zone, and ii) the Respiratory Zone [19]. The role of the Conducting Zone is to move air, and consists of respiratory structures whose main purposes are for cleaning, warming, and humidification [19]. The Respiratory Zone on the other hand comprises of respiratory structures in the lungs that ensure proper gas exchange occurs [19]. An illustration of the respiratory system is shown in Figure 2.1. In the context of mechanical ventilation as well as this thesis, the physiology related to breathing occurring in the lower respiratory tract (trachea to alveoli) is of primary interest. 


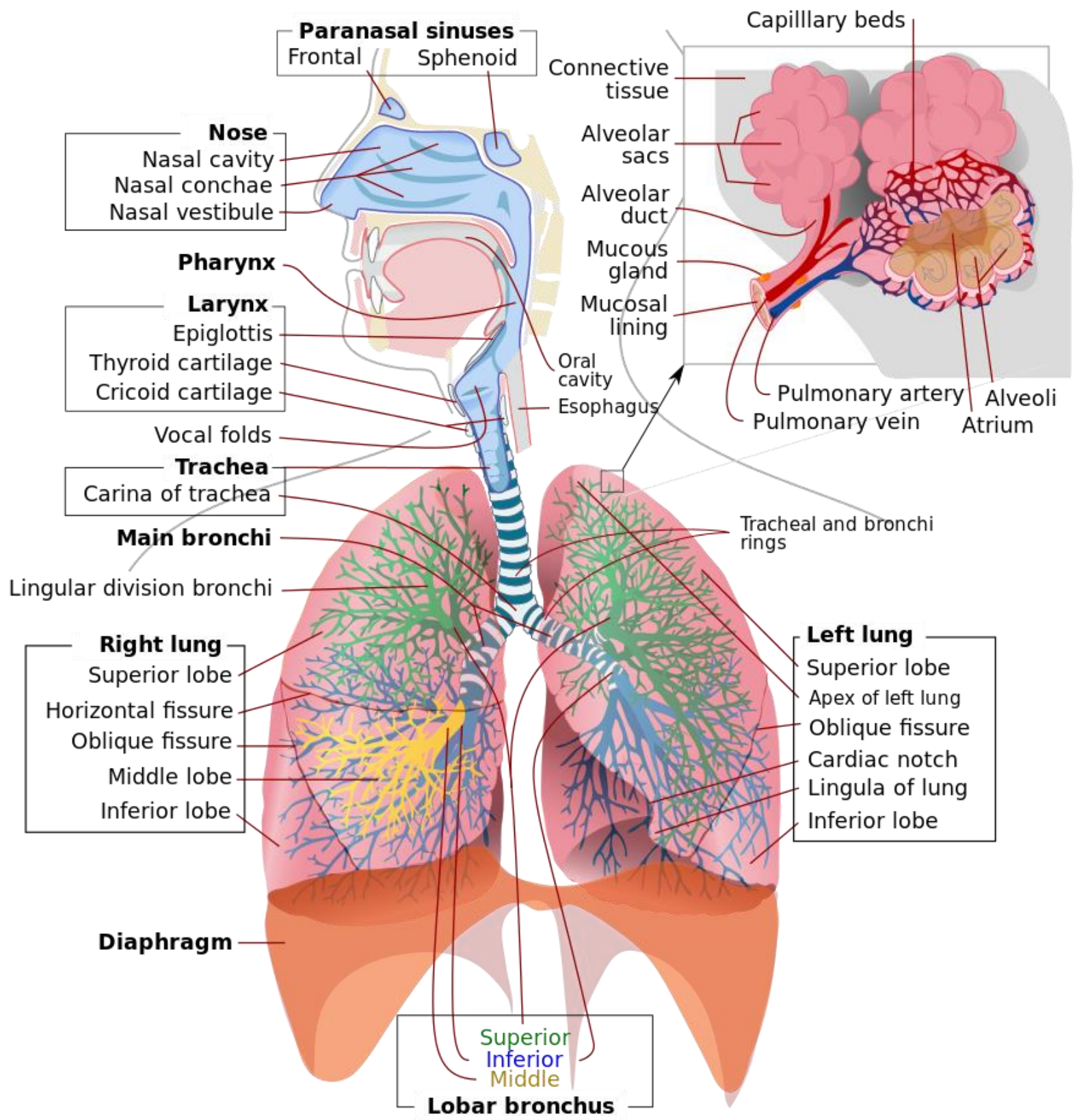

Figure 2.1. The anatomical structures of the respiratory system, which includes the Conducting Zone (nose to bronchial tree) and the Respiratory Zone (lungs and alveoli). The Diaphragm lies inferior to the lungs. Source: [20] 


\subsubsection{Anatomical Structures Involved in Breathing}

\section{Trachea and Bronchial Tree}

The trachea, which is also known as the windpipe, and the bronchial tree are parts of the Conducting Zone. The trachea is a large membranous tube that consists of dense regular connective tissue and C-shaped hyaline cartilage [19]. The trachea splits into two smaller tubes known as the main bronchi. The two main bronchi branch off into several generations of smaller bronchi, resulting in a tree-like structure. The respiratory passageways after the trachea are known as the bronchial tree, which ultimately gives rise to the alveoli [19].

\section{Alveoli}

The alveoli are part of the Respiratory Zone. Alveoli are small, air-filled chambers, which are the site of gas exchange between the air and the blood. The alveoli walls are lined with elastic fibres that allow for the expansion and recoil of the alveoli structure during ventilation.

\section{Lungs}

The lungs are the primary organs of respiration and reside in the thoracic cavity. The lungs house the structures of the Respiratory Zone, such as the alveoli, as well as parts of the Conducting Zone like the bronchial tree. In humans, the lungs come as a pair, and are divided into several lobes; the right lung consists of three lobes while the left lung has two lobes as well as an indentation known as the cardiac notch. The cardiac notch allows for the heart to lie in between the two lungs. The blood supply of the lungs consists of two major routes: i) the pulmonary circulation, which converts deoxygenated blood into oxygenated blood through gas exchange, and ii) bronchial circulation, which provides a blood supply to the airways of the lungs (i.e. bronchial tree) [19]. 


\section{Thoracic Cavity and Muscles of Respiration}

The thoracic cavity encloses the space between the thoracic walls and the diaphragm. The diaphragm and muscles of the thoracic walls are responsible for ventilation. During ventilation, the shape of the thoracic cavity must change to facilitate the expansion and recoil of the lungs.

\section{$\underline{\text { Muscles of Inspiration }}$}

The muscles of inspiration include the diaphragm, external intercostals, pectroalis minor, and scalenes [19]. The diaphragm, which is a dome-shaped muscle located inferior to the lungs as shown in Figures 2.1 and 2.2, is considered to be the main muscle of inspiration. During inspiration, the diaphragm contracts and pushes down in the inferior direction, which results in an increase in the thoracic volume. The other muscles listed in this section are responsible for elevating the ribs [19].

\section{Muscles of Expiration}

The role of the muscles of expiration are to lower the ribs and sternum [19]. Muscles of expiration include the internal intercostals and the transverse thoracis [19].

\section{Pleura}

Pleural serous membranes surround the lungs to form the pleural cavity. The visceral pleura is attached to the surface of the lungs while the parietal pleura connects to the inner thoracic wall and the superior surface of the diaphragm. The pleural cavity contains pleural fluid that holds the visceral and parietal pleura together, which allows the lungs to adhere to the thoracic walls. Additionally, the pleural fluid also ensures that the visceral and parietal pleura are able to move with each other when the shape of the lungs and the thoracic cavity change during a breathing cycle. The pleural membranes and their fluids provide lubrication to the lungs to minimize friction 
during expansion and contraction, and ensure that the structures of the lungs are protected from other thoracic structures. An illustration of the pleural membranes and the pleural cavity is shown in Figure 2.2.

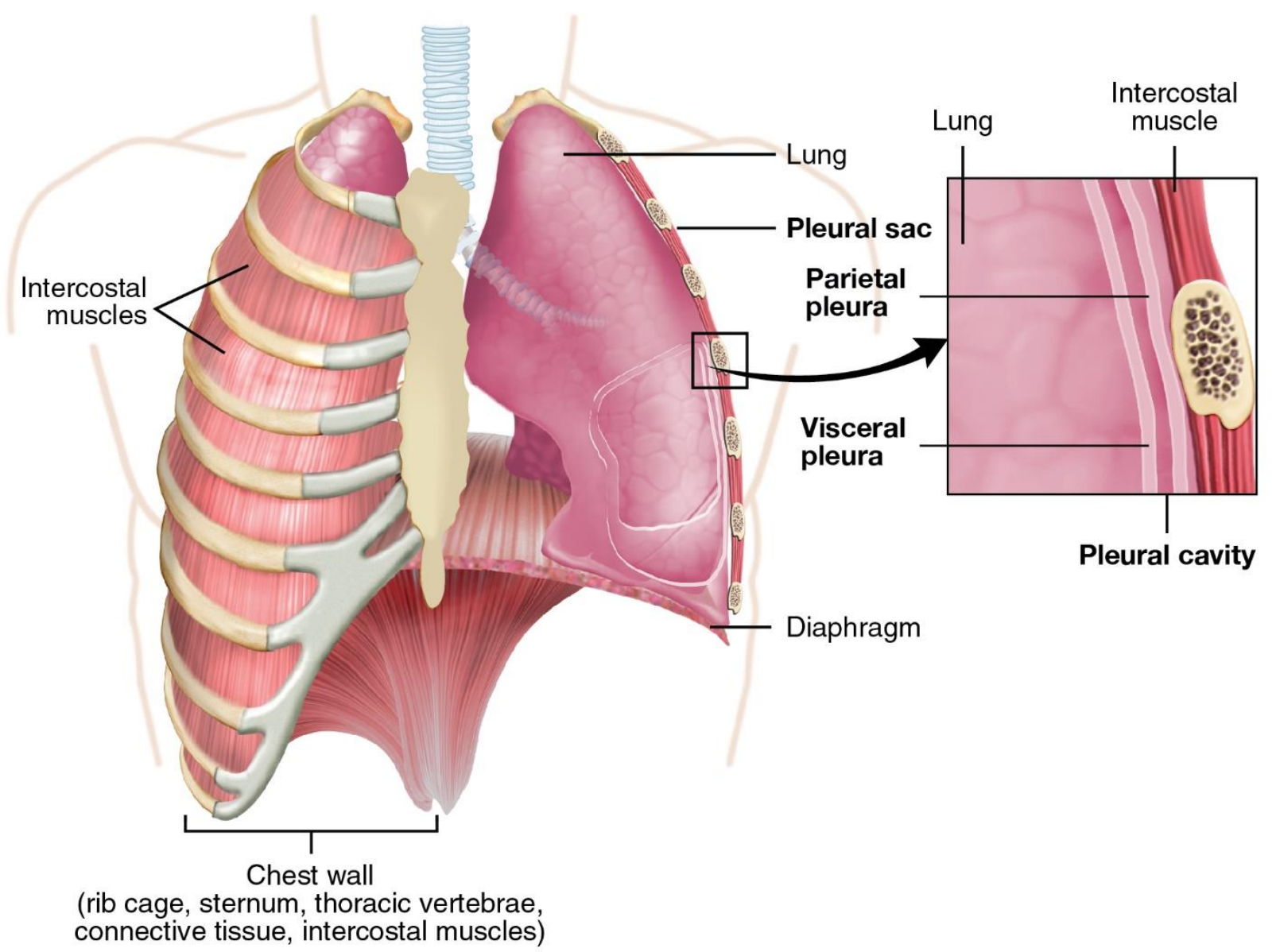

Figure 2.2. The anatomical structures of the chest wall and muscles of respiration, and a visual representation of the pleural membranes and the pleural cavity. Source: [21] 


\subsubsection{Ventilation}

\section{Relationship between Pressure, Airflow, and Volume}

Recall that ventilation refers to the movement of air into and out of the lungs. In order for airflow to occur, a pressure gradient must exist between the atmosphere outside of the body (i.e. barometric pressure, $\left.\mathrm{P}_{\mathrm{B}}\right)$ and the alveoli $\left(\mathrm{P}_{\mathrm{alv}}\right)$. In the case of inspiration, the pressure within the alveoli must be less than the atmospheric pressure to facilitate air movement into the lungs. The reverse is true for expiration; the pressure within the alveoli must be greater than the atmospheric pressure, which results in airflow out of the lungs. The pressure gradient is generated by changes in thoracic volume according to Boyle's Law:

$$
P \propto \frac{1}{V}
$$

\section{Physiology of Breathing}

During inspiration, the volume of the thorax increases due to the respiratory muscles contracting, resulting in the inflation of the lungs. The volume of the alveoli increases, which leads to a decrease in $\mathrm{P}_{\mathrm{alv}}$ due to the inverse relationship between volume and pressure as shown in EQ2.1. Thus, $P_{\text {alv }}<P_{B}$, which results in air flowing from outside the body and into the lungs. During expiration, the respiratory muscles relax and deflate the lungs. A decrease in lung volume therefore results in a decrease in alveolar volume and an increase in $\mathrm{P}_{\mathrm{alv}}$. The relationship between alveolar pressure and barometric pressure is therefore $P_{a l v}>P_{B}$, indicating that the pressure gradient has been reversed. The flow of air changes direction and moves from the alveoli to the outside of the body. 


\section{Factors Affecting Alveolar Volume}

Alveolar volume plays a significant role in breathing, because the direction of the pressure gradient is dictated by changes in the alveolar volume. According to [19], there are two key factors that affect how alveolar volume changes:

\section{$\underline{\text { Lung Recoil }}$}

The lungs exhibit a property known as elastic recoil, which is the tendency for the lungs to decrease in size as they are stretched. When alveoli are expanded, their tendency to recoil is due to the elastic fibres lining their walls, as well as surface tension caused by the fluid film surrounding the them.

\section{$\underline{\text { Pleural Pressure }}$}

The parietal and visceral pleura encapsulates the lungs to form the pleural cavity. The pressure within the pleural cavity is known as pleural pressure $\left(\mathrm{P}_{\mathrm{pl}}\right)$. Under normal circumstances, pleural pressure is less than alveolar pressure (i.e. $P_{p l}<P_{a l v}$ ), which allows for the alveoli to remain expanded. The relationship between $\mathrm{P}_{\mathrm{pl}}$ and $\mathrm{P}_{\mathrm{alv}}$ can be explained by the suctioning effect within the pleural cavity; due to lung recoil, the visceral pleura pulls away from the parietal pleura, which results in a negative pressure in $\mathrm{P}_{\mathrm{pl}}$.

\subsection{Respiratory Monitoring \& Guiding Mechanical Ventilation}

As mentioned in Chapter 1, there has been interest in the critical care community to develop new "lung-protective" mechanical ventilation strategies to overcome challenges such as Ventilator-Induced Lung Injuries (VILI) resulting from the following mechanisms [22]-[23]:

- Barotrauma: excessive inflation pressure, 
- Volutrauma: overdistension of lung,

- Atelectrauma: injury due to shear forces caused by cyclic opening and collapse of lung units, and

- Biotrauma: injuries due to inflammatory response to mechanical lung injury

To guide mechanical ventilation interventions and avoid the risks of the abovementioned mechanisms, several clinical parameters are monitored during treatment.

\subsubsection{Respiratory Monitoring in Mechanical Ventilation}

In the clinical context, the Airway Pressure $\left(\mathrm{P}_{\mathrm{aw}}\right)$ is universally acquired from mechanically ventilated patients by the mechanical ventilator to monitor the patient's respiratory mechanics [24]. Airway Pressure is representative of the distending pressure of the respiratory system, which is the amount of force required to inflate the respiratory system. Airway Pressure consists of two main components: i) Transpulmonary Pressure $\left(\mathrm{P}_{\mathrm{L}}\right)$, which describes the insufflation of the lungs, and ii) Pleural Pressure $\left(\mathrm{P}_{\mathrm{pl}}\right)$, which moves the chest wall and can be mathematically expressed as a sum of $\mathrm{P}_{\mathrm{L}}$ and $\mathrm{P}_{\mathrm{pl}}$ according to EQ2.2 [18], [25]:

$$
P_{a w}=P_{L}+P_{p l} \quad \text { (EQ2.2) }
$$

While $\mathrm{P}_{\mathrm{aw}}$-based interpretations were originally used to guide mechanical ventilation in patients, it has been found that $\mathrm{P}_{\mathrm{aw}}$ can be significantly influenced by factors such as breathing patterns, alterations in lung volume, and varying characteristics of chest wall elastance [26]. In fact, the chest wall elastance of mechanically ventilated patients suffering from pathological conditions such as Acute Respiratory Distress Syndrome (ARDS) can vary greatly among individuals [26][29]. Thus, differences in chest wall elastance makes interpretation of $\mathrm{P}_{\mathrm{aw}}$ (and by extension, the respiratory mechanics) more difficult. Additionally, the lungs and chest wall are both elastic 
structures that are set up in a series connection [26]. This means that a portion of the pressure delivered by the ventilator to the respiratory system is distributed between the component distending the lung $\left(\mathrm{P}_{\mathrm{L}}\right)$ and the component distending the chest-wall $\left(\mathrm{P}_{\mathrm{pl}}\right)$, which also contributes to the difficulty of guiding mechanical ventilation solely through $\mathrm{P}_{\mathrm{aw}}[26]$, [30]. Because varying chest wall elastance in critically ill patients has clinical implications, as well as the fact that ventilator-delivered pressure is dissipated between the chest-wall and the lungs, it has been suggested that lung-protective mechanical ventilator strategies incorporate Transpulmonary Pressure parameters in order to monitor the patient's lung mechanics [26], [31].

\section{Significance of Transpulmonary Pressure in Guiding Mechanical Ventilation}

The Transpulmonary Pressure has been used to describe the pressure distending the lung [18],[25], [31]-[32]. In other words, $\mathrm{P}_{\mathrm{L}}$ is the amount of pressure required to drive ventilation in the lungs. Rearranging EQ2.2, $\mathrm{P}_{\mathrm{L}}$ can be calculated as the difference between the distending pressure of the respiratory system, $\mathrm{P}_{\mathrm{aw}}$, and the pressure required to distend the chest-wall, $\mathrm{P}_{\mathrm{pl}}$, as shown in EQ2.3:

$$
P_{L}=P_{a w}-P_{p l}
$$

The use of Transpulmonary Pressure to guide lung-protective mechanical ventilation strategies has gained attention in recent years, because it is able to determine the amount of pressure required to keep the lungs open during mechanical ventilation, can be used to estimate inspiratory effort, and measures the mechanical stress of the lungs [18], [33]. The aforementioned factors are important to consider in tailoring and managing mechanical ventilation settings for individual patients in order to optimize gas exchange while avoiding or mitigating the mechanisms leading up to VILI [26], [29]. 


\section{Esophageal Pressure as Surrogate for Pleural Pressure}

In the definition of Transpulmonary Pressure shown in EQ2.3, the Pleural Pressure is used to subtract the pressure component of the chest-wall. The Pleural Pressure, as noted in Section 2.1.2, is the pressure within the pleural cavity, which suggests that a pressure sensor must be inserted directly into the pleural space. However, insertion of a device into the pleural cavity to measure $\mathrm{P}_{\mathrm{pl}}$ is virtually impossible as it comes with the risk of inducing a pneumothorax (i.e. collapsed lung) in the patient due to puncturing the pleural membranes [19], [34]. As a result, Esophageal Manometry has been used as a non-invasive approach to acquire the Esophageal Pressure (Peso), which acts as a suitable surrogate for $\mathrm{P}_{\mathrm{pl}}$ [18], [30]-[31], [35].

\section{Significance of Esophageal Pressure in Guiding Ventilation}

The direct measurement of Esophageal Pressure as a surrogate for $\mathrm{P}_{\mathrm{pl}}$ is one approach to estimating $\mathrm{P}_{\mathrm{pl}}$ and plays a significant role due to its ability to distinguish between the distending pressures of the lungs and the chest wall [29], [30]. While measuring the Esophageal Pressure in mechanically ventilated patients is not a new approach, it is seldomly used in the clinical context and is instead used more as a research tool [18]. The reason for its lack of use in the clinic is due to questions surrounding the validity of Esophageal Manometry [30]. Concerns such as insertion and placement of the pressure sensor via an esophageal catheter, the interpretation of the Peso trace, and the accuracy of Peso measurements arise [26], [30]-[31]. Despite the Peso's limitation, a landmark study by Talmor et al. [14] used the Peso as a surrogate for $\mathrm{P}_{\mathrm{pl}}$ to estimate $\mathrm{P}_{\mathrm{L}}$. It was found that it was possible to make repeated measurements of Peso with a high degree of fidelity and quality to help guide mechanical ventilation. Furthermore, using Peso to adjust $\mathrm{P}_{\mathrm{L}}$ also resulted in significant improvements in both blood oxygenation and pulmonary compliance (i.e. the ability for the lungs to stretch) in patients suffering from ARDS [14]. 
In addition to guiding mechanical ventilator strategies, the Peso signal can also be used for other clinical applications including [18], [31]:

- Gaining insights into the pathophysiology of respiratory failure,

- Titrating the level of respiratory and pharmacological support,

- Detecting asynchrony leading to poor patient-ventilation interactions,

- Assessing patient inspiratory efforts, and

- Aiding in ventilator weaning.

The abovementioned applications of the Peso show the importance of its use and the knowledge derived from it in managing treatment options for critically-ill patients.

\section{Acquisition of Esophageal Pressure}

According to [24], [31], and [35], the Peso signal is acquired through a pressure transducer via an air-filled balloon connected to the distal end of a catheter during mechanical ventilation. The balloon catheter is inserted either through the nostril or the mouth and positioned in the lower third of the esophagus. In addition to Peso, the $\mathrm{P}_{\mathrm{aw}}$ and the Flow are also acquired during invasive mechanical ventilation. The analog data from Peso, $\mathrm{P}_{\mathrm{aw}}$, and Flow are converted into digital data using a BIOPAC system and stored as raw data in a workspace. The procedure for catheter insertion and signal validation is described in detail in [24], [31], and [35]. The clinical setup for signal acquisition in mechanically ventilated patients is shown in Figure 2.3 and a sample of a Peso as a function of time is shown in Figure 2.4. 


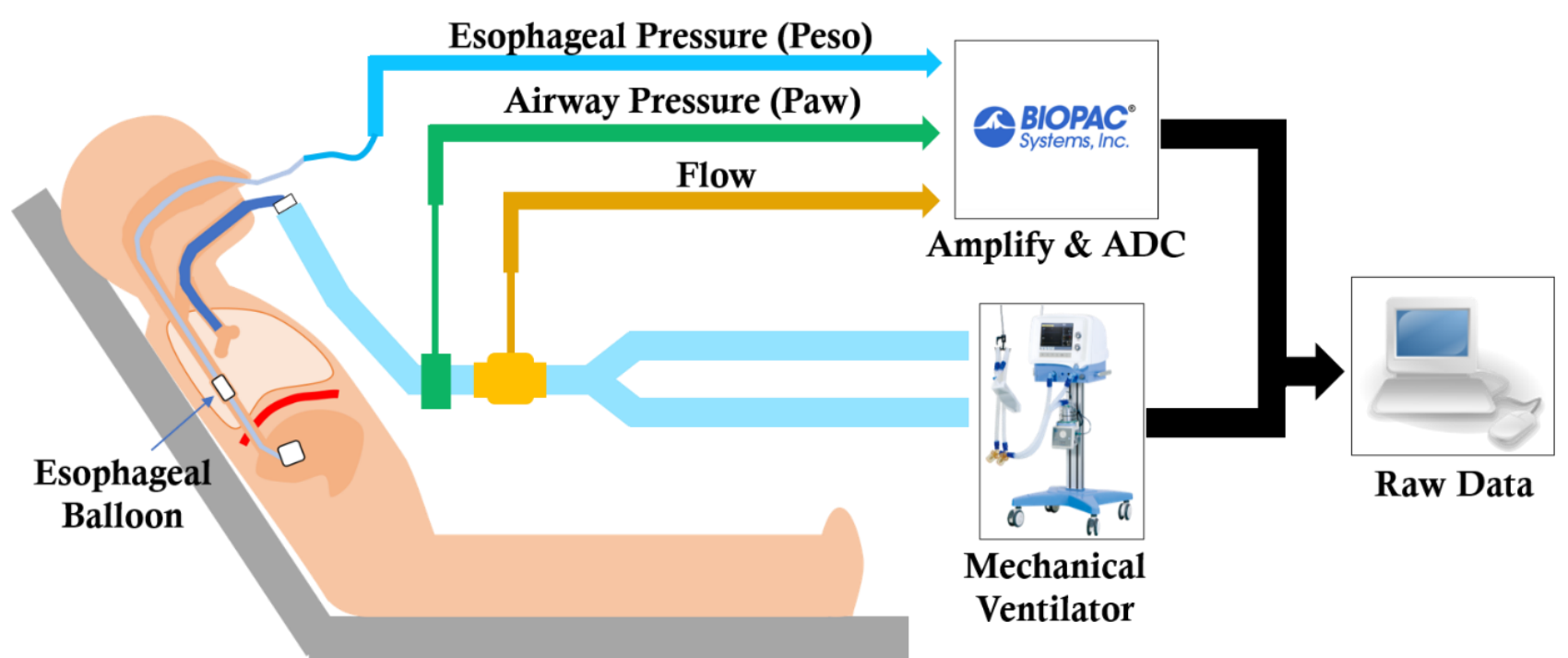

Figure 2.3. Clinical setup used to acquire data from mechanically ventilated patients. Source: Adapted from Dr. Laurent Brochard's iBEST 2017 poster.

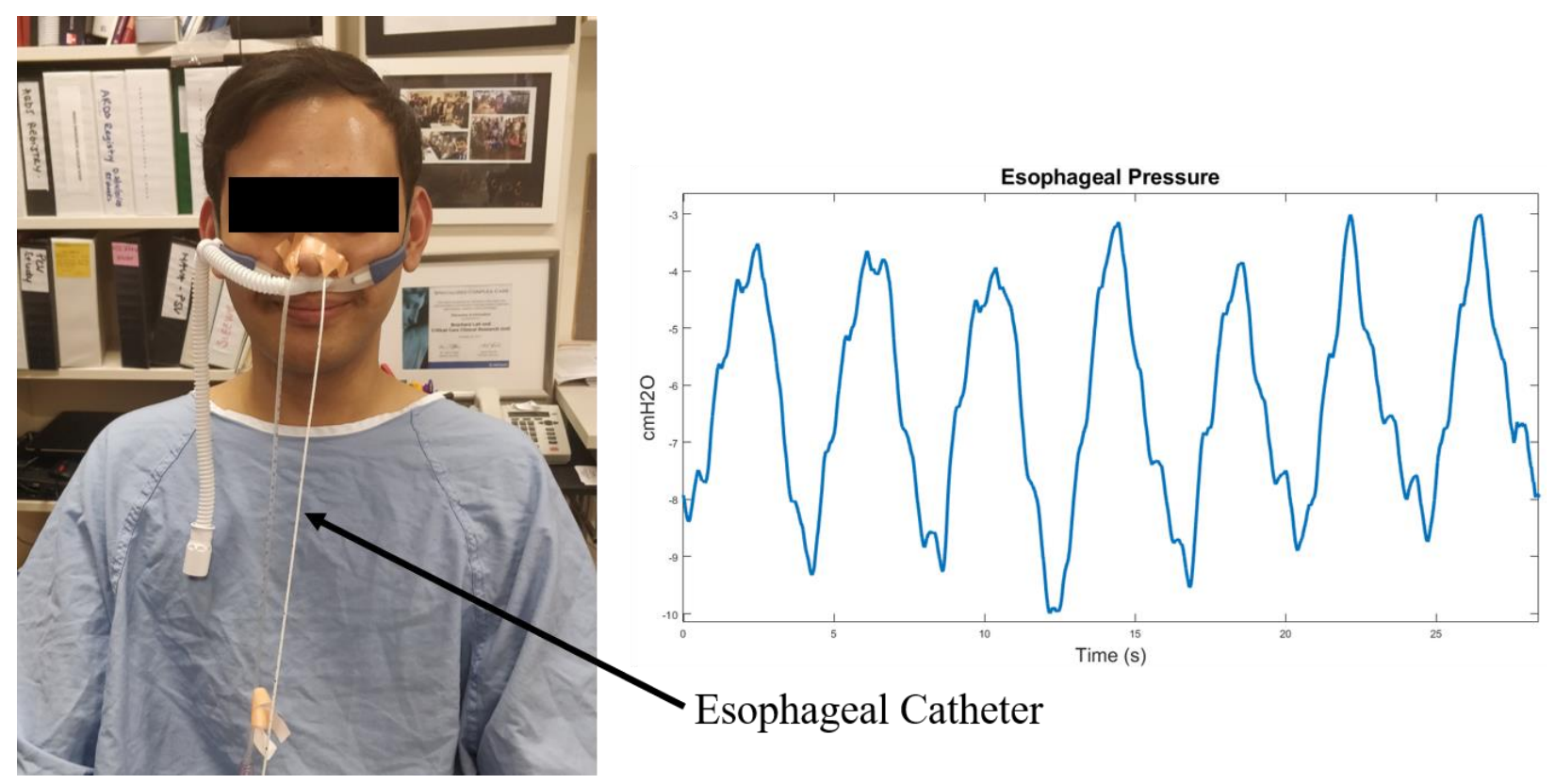

Figure 2.4. Nasal insertion of esophageal catheter (left) and the corresponding Peso signal (right). 


\subsection{Characteristics of the Esophageal Pressure (Peso) Signal}

\subsubsection{Time-Domain Characteristics of Peso}

\section{Overview of the Time-Domain}

The time-domain representation of a signal describes the variation in amplitude of the data or measurement as a function of time. The changes in amplitude are an important aspect of the time-domain, because the variations carry information of the processes being measured or observed. In the context of biomedical signals, the amplitude variations are a direct result of the underlying physiological processes.

Many of the physiological processes in the human body, such as breathing or the heart beating, are rhythmic in nature. The rhythmic nature of these processes manifests themselves as quasi-periodic (i.e. irregularly periodic) waveforms or traces, which have distinguishing shapes/morphology. The resulting morphology generated by the amplitude changes in the biomedical signal can then be mapped to specific physiological occurrences or events. Two popular examples that can be used to visualize the idea of connecting a physiological event to a corresponding morphology are: i) the positive and negative inflections of a respiratory signal, which represents inhalation and exhalation respectively, and ii) ventricular depolarization represented by the QRS complex of an electrocardiogram (ECG), which results in the contraction of the ventricular chambers of the heart. From these two examples, visualizing a physiological process in the form of a biomedical signal can be helpful, particularly for clinicians who are monitoring and treating their patients. 


\section{Time-Domain Characteristics of the Raw Peso Signal}

\section{"Active Breathing" Conditions}

As described in Section 2.1.2, the process of inspiration and expiration depends on the pressure gradient generated between the lungs and the body surface/environment by the respiratory muscles, which occurs during an "active breathing" condition. Under "active breathing" conditions, a person is able to recruit their respiratory muscles to initiate an effort to take a breath. Since the Peso is used as a surrogate for $\mathrm{P}_{\mathrm{pl}}$, the amplitude of the Peso signal should follow Boyle's Law according to the relation shown in EQ2.4:

$$
P(t) \propto \frac{1}{V(t)}
$$

It is important to remember that the pressure $(\mathrm{P})$ and volume $(\mathrm{V})$ of the pleural cavity are both functions of time. To visualize the time-series represented by the Peso signal, a time-domain representation of the Flow and corresponding Peso signal from a mechanically ventilated, spontaneously breathing (i.e. some degree of active inspiratory effort - a form of "active breathing") patient who is receiving pressure support is shown in Figure 2.5. Some significant clinical time-domain parameters related to pulmonary physiology are also included in the figure. Note that the parameters illustrated on the figure is not an exhaustive list. To draw the connection between Boyle's Law, the flow provided by the mechanical ventilator, the Peso for a spontaneously breathing patient, the clinical parameters, and the physiological meaning of the clinical parameters shown in Figure 2.5, it is best to describe the parameters chronologically from left to right (1) to (3) for each of the three breaths/epochs as depicted in the illustration. 


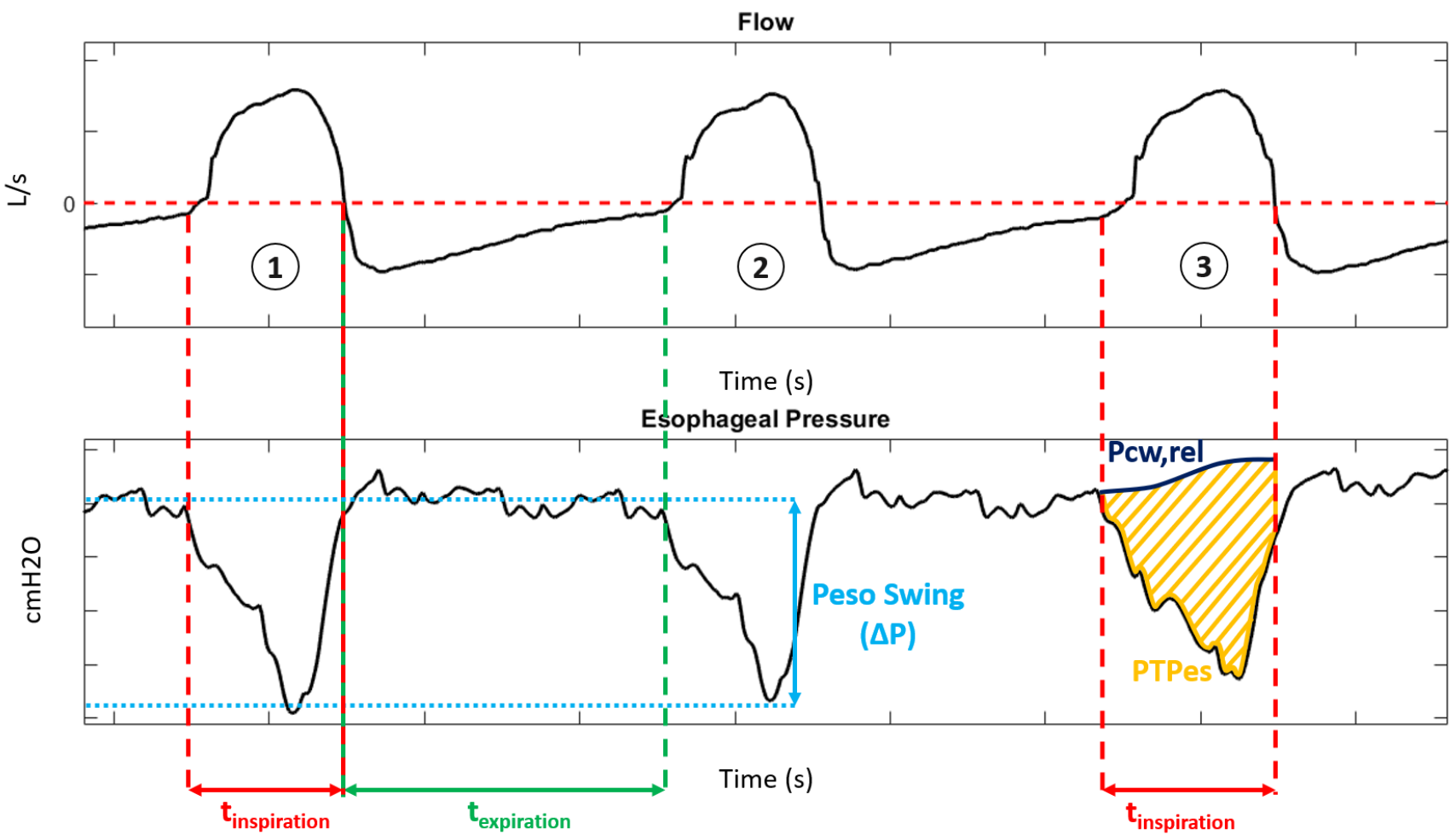

Figure 2.5. The time-domain representation of the Flow signal (top) and Esophageal Pressure/Peso signal (bottom). The horizontal dashed red line in the Flow signal indicates the threshold between mechanical insufflation and mechanical exsufflation. Clinical parameters in the Peso signal are illustrated in the figure above. In the breath designated by (1), the vertical red dashed lines indicate the total inspiration time $\left(\mathrm{t}_{\text {inspiration }}\right)$ and the green vertical dashed line represents total expiration time (texpiration). In (2), the blue horizontal dotted lines and arrow show the Peso Swing $(\Delta \mathrm{P})$. In (3), the esophageal pressure-time product (PTPes) is highlighted in orange. The dark blue curve represents the chest wall pressure during a relaxed state (Pcw,rel), which is obtained during passive breathing. The small pressure fluctuations caused by the beating heart known as cardiogenic oscillations (CGOs), which are not labelled, are present throughout the Peso signal. 
Before describing each of the three epochs in Figure 2.5, there are two notable characteristics of the plots to note:

1) The red horizontal dashed lined in the Flow plot: The dashed line lies on the zero-value, which is the threshold used to distinguish between mechanical insufflation and mechanical exsufflation. When the plot of flow is positive (i.e. greater than zero), mechanical insufflation is occurring, which means that the mechanical ventilator is pushing air into the patient for the inspiration process. When the plot of flow is negative (i.e. less than zero), the mechanical ventilator switches to mechanical exsufflation to perform expiration.

2) The starting point of inspiration time $\left(t_{\text {inspiration }}\right)$ : Although the horizontal dashed red line in the Flow plot determines when mechanical insufflation and exsufflation occurs, the onset of inspiration does not begin when the Flow signal is positive. The reason for this is because Figure 2.5 is an illustration of a spontaneously breathing patient, which means that the patient can recruit their respiratory muscles to make some degree of inspiratory effort. Thus, the start of inspiration begins before the flow is positive as shown by the vertical lines, which represents

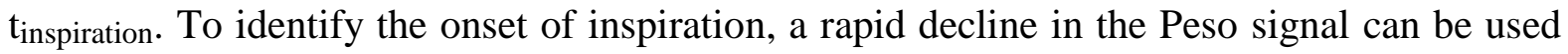
[36], which indicates that the patient has made an effort to take a breath.

During the inspiration phase of (1), the respiratory muscles are contracting over a period of time, which is illustrated by red vertical dashed lines and denoted as $t_{\text {inspiration. }}$ As the respiratory muscles contract, the volume of the chest cavity increases to allow for the expansion of the lungs. An increase in the chest cavity volume and pleural cavity generates a pressure gradient between the body surface and the alveoli, which allows for air to flow into the lungs. A negative deflection in the Peso signal is therefore observed due to two factors: i) the pressure is inversely proportional 
to volume according to Boyle's Law (EQ2.4), and ii) the suctioning effect of the pleura caused by pleural fluid. During expiration, which is characterized by green vertical dashed lines and denoted by ( $\left.t_{\text {expiration}}\right)$, the respiratory muscles are relaxing over time. The relaxation of the muscles decreases the volume of the chest cavity in order to deflate the lungs, which results in a positive deflection in the Peso as well as the exhalation of $\mathrm{CO}_{2}$.

In (2), the Peso swing $(\Delta \mathrm{P})$ is shown as the difference between the two horizontal dotted light blue lines. To put the Peso swing into a clinical context, $\Delta \mathrm{P}$ describes the amplitude of the esophageal pressure during a breathing cycle. The significance of $\Delta \mathrm{P}$ is that it can be used to give clinicians an idea of their patient's breathing effort [18], [31], [37]. Although using $\Delta \mathrm{P}$ is a relatively simple and convenient way of quantifying a patient's breathing effort, there are several limitations that the Peso swing does not consider, such as duration of the respiratory muscle contractions as well as frequency of breaths [37]. It has also been suggested that $\Delta \mathrm{P}$ by itself has a poor correlation with energy expenditure of the respiratory muscles and can therefore not be used as a parameter to estimate the amount of energy required to drive respiration [37]. Nevertheless, since $\Delta \mathrm{P}$ can be monitored at the bedside in real-time, it can still be used to get a general trend of the patient's breathing effort [37].

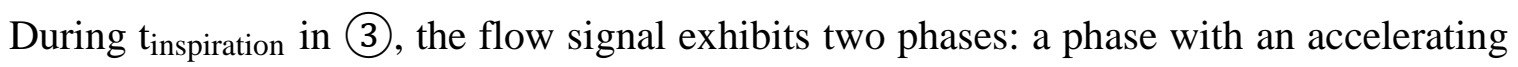
flow at the beginning and a second phase which describes the decelerating flow, both of which are provided by the mechanical ventilator. In ventilation modes such as pressure-support ventilation (PSV), a rapid increase in flow during $t_{\text {inspiration }}$ is necessary to achieve the pre-defined level of pressure [38]. Once the target pressure is achieved, less flow of air into the lungs is needed to maintain the pressure, which results in the decelerating flow provided by the ventilator. 
The portion in the Peso swing highlighted in orange represents a parameter known as the esophageal pressure-time product (PTPes), which can be used to estimate the energy expenditure and oxygen consumption of the respiratory muscles, hence the reason it occurs only in the duration of tinspiration [18], [31]. The PTPes can also be used as a means to quantify a patient's breathing effort, which can be useful in monitoring patients in the ICU with poor patient-ventilation interactions as well as patients who are difficult to wean [37]. In order to estimate the PTPes, it is necessary to first determine the muscle pressure $\left(\mathrm{P}_{\text {mus }}\right)$, which is the pressure produced by the respiratory muscles during active inspiration. For a spontaneously breathing patient, $\mathrm{P}_{\text {mus }}$ is defined according to EQ2.5 as the difference between the static recoil pressure of the chest wall (shown in dark blue in Figure 2.5) and the Peso under active conditions [18], [31]:

$$
P_{m u s}=P_{c w, r e l}-P_{e s o} \quad(\text { EQ2.5) }
$$

The static recoil pressure of the chest wall $\left(\mathrm{P}_{\mathrm{cw}, \mathrm{rel}}\right)$ can be measured by recording the Peso signal under passive conditions (i.e. no patient effort during breathing) [18]. With knowledge of $\mathrm{P}_{\text {mus, }}$, the PTPes can then be calculated as the integral of $\mathrm{P}_{\text {mus }}$ over the duration of $\mathrm{t}_{\text {inspiration }}$ as shown in EQ2.6 [18], [31]:

$$
\text { PTPes }=\int P m u s \cdot d t \quad(\mathbf{E Q 2 . 6 )}
$$




\section{"Passive Breathing" Conditions}

In the clinical context, another breathing condition or mode is "passive breathing." During "passive breathing" conditions, patients do not provide any inspiratory effort during inspiration; respiration is achieved solely by the mechanical ventilator. To achieve the "passive breathing" condition, patients are sedated and a neuromuscular blocking agent (NMBA) (i.e. a reversible paralysis-inducing drug) is administered to their respiratory muscles [39]. The use of sedatives in patients receiving mechanical ventilation support in ICUs is intended to relieve pain and discomfort caused by respiratory treatments or interventions [40]. Treatment plans for patients with mild to severe acute respiratory distress syndrome (ARDS) often include the use of NMBAs in conjunction with sedatives during the early phases of treatment to improve oxygenation, and ultimately, overall survival rates [39], [41].

The distinction between "passive breathing" and "active breathing" conditions is important to make, because the morphology of the Peso changes depending on the condition. While the Peso signal deflects in the negative direction for "active breathing" conditions during inspiration as seen in the previous section, the opposite is true for "passive breathing" conditions; the Peso will deflect in the positive direction as shown in Figure 2.6. Since the NMBAs prohibit the contraction of the respiratory muscles, the mechanical ventilator performs inspiration by providing a positive pressure to the airways. The positive pressure allows air to flow into the lungs, thereby resulting in a positive deflection in the Peso signal. 


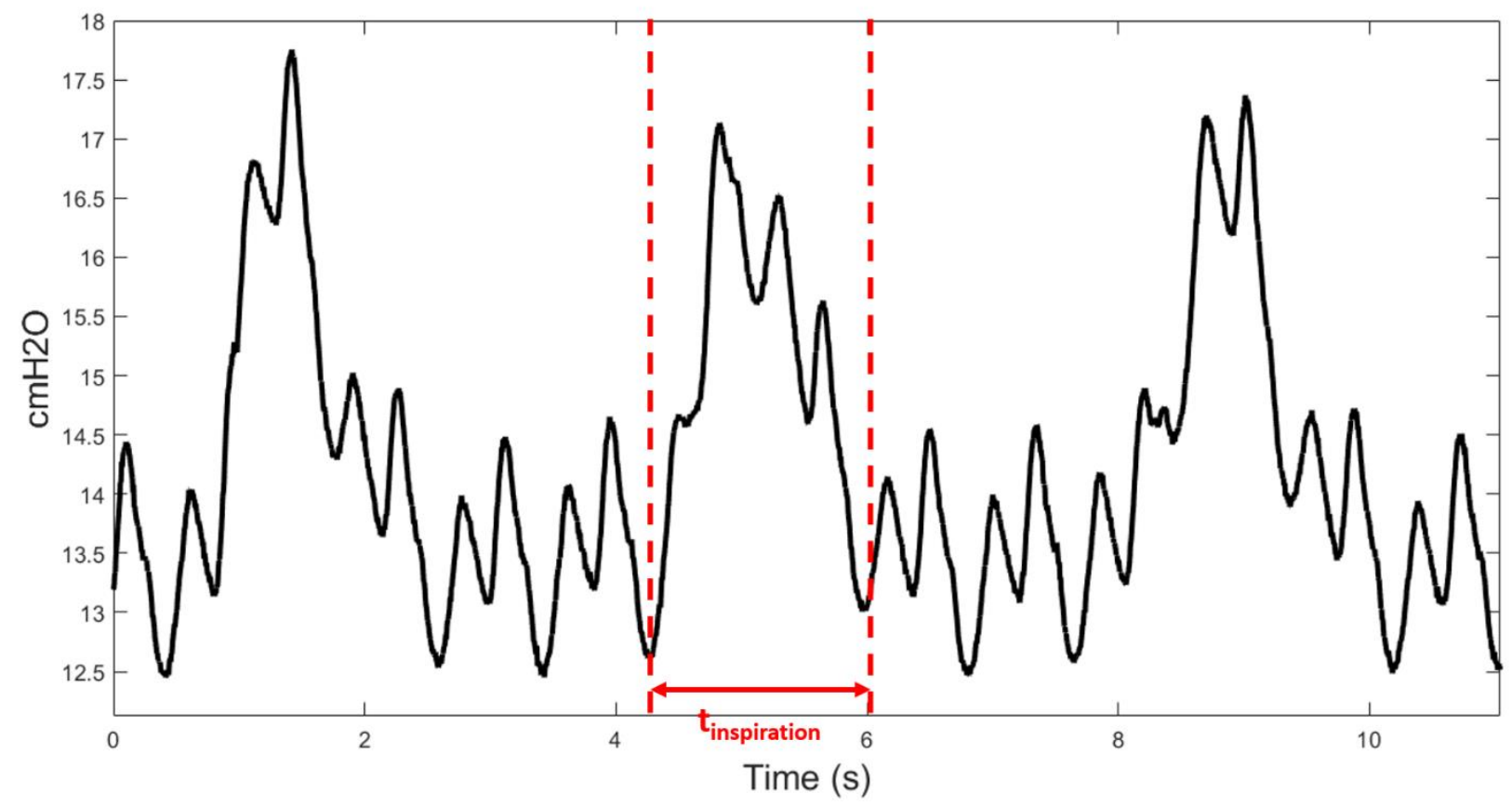

Figure 2.6. A Peso signal from a passively breathing patient. The red vertical dashed lines represent the duration of one inspiratory cycle. At the onset of inspiration, the mechanical ventilator provides positive pressure to allow airflow into the lungs resulting in the positive deflection of the Peso signal. 


\subsubsection{Frequency-Domain Characteristics of Peso}

\section{Overview of the Frequency-Domain}

The frequency-domain is another common representation of a signal, which describes the signal's content as a function of frequency. In other words, the frequency-domain can express how much of the signal appears in a specific frequency band. The frequency-domain representation of a signal is often referred to as either the frequency spectrum or the power spectrum. While the plots generated in time-domain can be intuitive and easier to understand due to its relation to the physical processes being observed over time, the frequency/power spectrum plots generated in the frequency-domain can provide additional insights to the signal from a different perspective. Furthermore, there are instances where the time-domain signal can be complex and difficult to interpret; exploiting the frequency-domain representation may be far more helpful in extracting useful information in these cases.

In the context of biomedical signal processing, the frequency-domain can be helpful in accomplishing two goals:

1. Characterizing biomedical signals, and

2. Enhancing biomedical signals

Characterizing biomedical signals in the frequency-domain is an important aspect of biomedical signal processing and analysis, because information about the behaviour of the biomedical system

can be derived. As mentioned previously, the frequency-domain is used to get information about the frequency content of a signal, and for biomedical signals specifically, the frequency content typically corresponds to the physiological processes being measured from a specific organ system. Since many of the biomedical signals acquired from the human organ systems are quasi-periodic 
in nature, a finite bandwidth can then be defined for the processes measured from the corresponding organ. By defining a range of frequency bands, it is possible to not only characterize the usable bandwidth of the biomedical signal (i.e. where most of the power of the signal lies in the power spectrum), but to also detect different physiology-related events present in the signal. Knowledge of the frequency characteristics and properties of a specific biomedical signal can provide a baseline understanding of how the corresponding physiological system normally works and can therefore be used to detect or get an idea of whether an organ system may be in an abnormal/diseased state.

Signal quality enhancement is another crucial aspect of biomedical signal processing that benefits greatly from the use of the frequency/power spectrum. Biomedical signals that are acquired in the clinic are often contaminated with various types of noise and interference, which present themselves as amplitude fluctuations in the time-domain. The amplitude fluctuations caused by the noise or interference can make it difficult to interpret the physiological signals, especially if an important clinical parameter relies on the morphology of the signal. Since each biomedical signal can be characterized by a usable bandwidth, it is then possible to distinguish between signal and noise/interference through the power spectrum. With the knowledge of which bandwidths are relevant for analysis, commonly used filters such as low-pass, high-pass, and bandpass filters can be designed to remove frequency components that correspond to noise or interference.

\section{The Fourier Transform}

In order to bring an aperiodic signal from the time-domain to the frequency-domain, the Fourier Transform can be applied. According to Fourier, any time-domain signal can be represented as a superposition of sines and cosines of different frequencies. For biomedical signals, 
which are aperiodic in nature, the Fourier Transform is used to decompose the signal, $x(t)$, into its constituent frequencies, $X(\omega)$, using the sinusoids as basis functions in the form of complex exponentials. The frequency components can be calculated via the Fourier Transform according to equation EQ2.7:

$$
\begin{gathered}
X(\omega)=\int_{-\infty}^{\infty} x(t) e^{-j \omega t} d t \quad \text { (EQ2.7) } \\
\text { where } \omega=2 \pi f
\end{gathered}
$$

Based on EQ2.7, $X(\omega)$ may be complex in nature. To visualize and analyze the frequency-domain representation of the signal, it is helpful to plot the frequency spectrum, which consists of two components:

1) The magnitude spectrum, $|X(\omega)|$, which describes how much of a sinusoid with frequency $\omega$ is present in $x(t)$

2) The phase spectrum, $\angle X(\omega)$, which represents how the different sinusoids align with each other to form $x(t)$

Through the frequency spectrum, it is then possible to accomplish the two goals outlined in the previous section, namely to characterize and enhance biomedical signals.

\section{Frequency Characteristics of the Raw Peso Signal}

The raw Peso signal that is acquired from the body is mixture of the "true" Peso signal as well as other physiological processes such as the heart-beat. The beating heart manifests itself as cardiogenic oscillations (CGOs), which is the main source of interference in the raw Peso signal 
that the work in this this thesis aims to suppress. Under normal circumstances, the frequency range for the Peso signal is $0.17-0.67 \mathrm{~Hz}$ while the CGO has a frequency range of $0.8-2.5 \mathrm{~Hz}$ [42].

\subsubsection{Sources of Interference in the Peso Signal}

\section{Sources of Interference in the Measured Peso Signal}

\section{Cardiogenic Oscillations (CGOs)}

In Section 2.2.1, the Peso acquisition setup was described. Although the location of the esophageal catheter allows for a usable Peso signal to be acquired, the anatomical positioning also puts the catheter right behind the heart. Thus, small oscillatory pressure fluctuations caused by the beating heart known as CGOs are captured in addition to the Peso [43]. In the Peso signal shown in Figure 2.7 it is evident that significant CGO interference is present. In the same figure, one CGO period is highlighted in red.

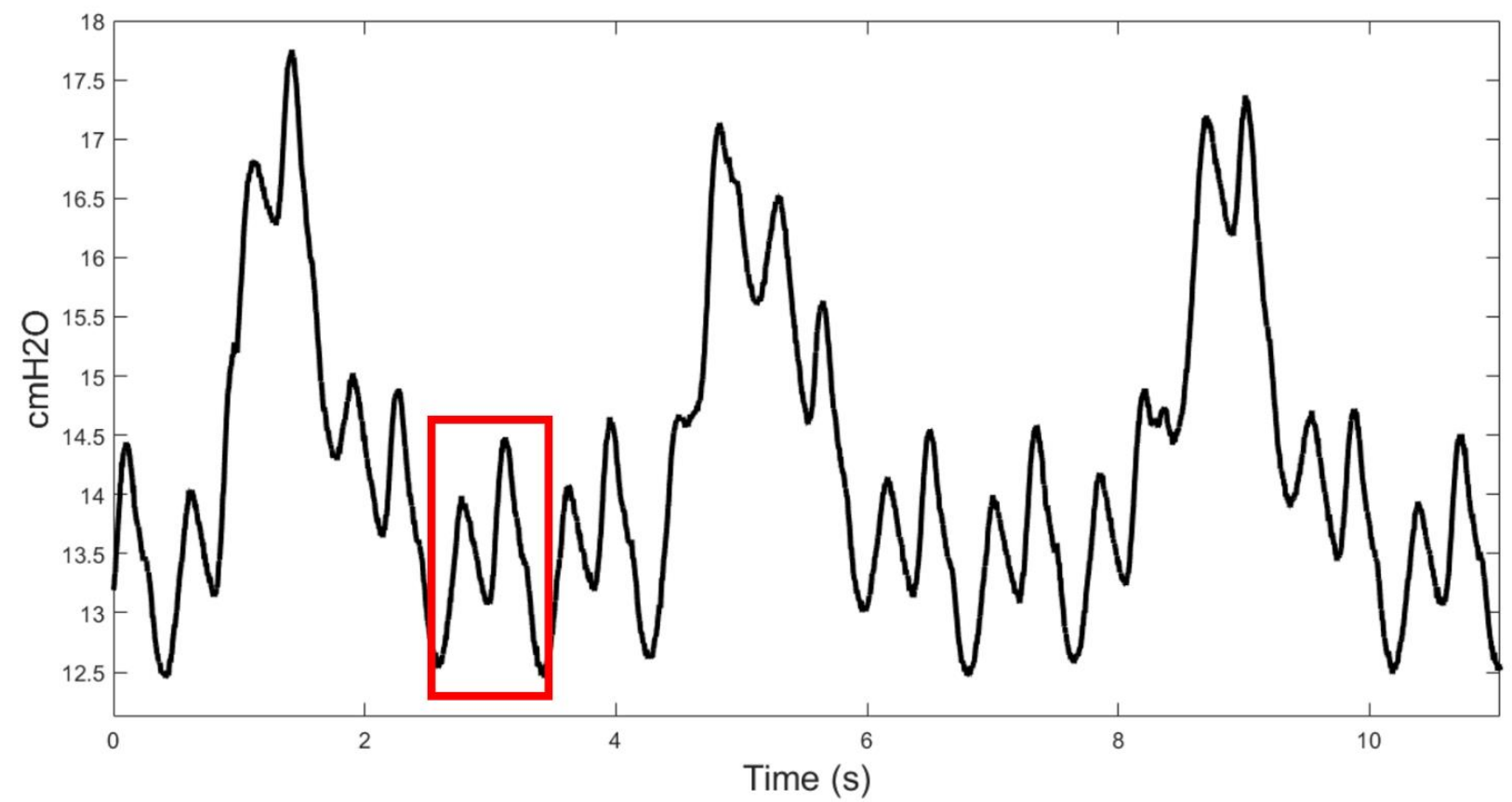

Figure 2.7. A Peso signal with significant CGO interference. A period of one CGO cycle is highlighted in red. 


\section{Esophageal Spasms}

Another major form of interference seen in the Peso signal are esophageal spasms caused by the act of swallowing and/or peristalsis. Esophageal spasms manifest themselves in the Peso signal as large spikes in pressure that are not related to the respiratory cycle as shown in Figure 2.8. According to [31], the presence of an esophageal spasm during a Peso measurement should be stopped and resumed only once the Peso returns to a stable range.

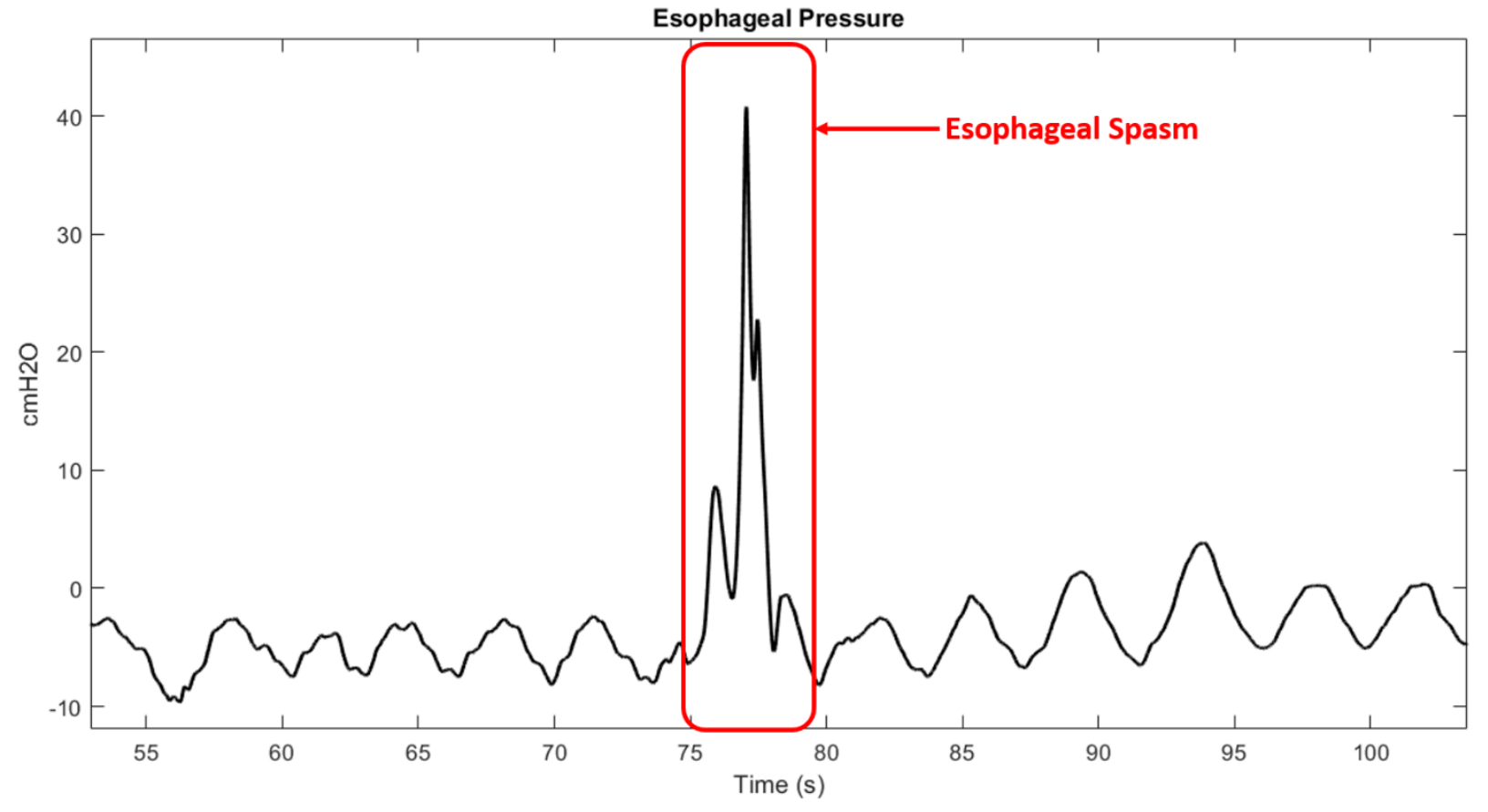

Figure 2.8. A Peso signal with an esophageal spasm occurring between $75-80$ seconds. The spasm is caused by a swallowing. 


\subsubsection{Relevant work related to CGO Suppression in Peso Signals}

Interference of CGOs in the Peso poses a problem during respiratory health monitoring, because the amplitude fluctuations may result in inaccurate estimates of clinical parameters [43][44]. Based on the frequency bandwidths of Peso and CGO provided in Section 2.3.2, it has been suggested that the frequency spectra for both signals may overlap [42], [44]. Because of the possible overlapping frequency spectra, conventional fixed filtering methods may not be appropriate for CGO removal/suppression.

Several research groups have developed approaches to suppress CGO from Peso signals. In [44] and their subsequent work in [45], the Peso was modelled as a sum of pressure swings caused by respiration and the CGO. To remove the CGO interference, an adaptive filtering technique using an adaptive noise-cancelling structure was proposed. The inputs to the proposed adaptive filter were the raw Peso signal and a corresponding ECG signal. An estimate of the CGO interference was created by obtaining the R-peaks in the ECG signal and applying a convolution with a linear dynamic system modelled by an impulse response. The estimated CGO was then subtracted from the Peso signal to obtain a CGO-free Peso signal. This technique was tested and validated on eight simulated patients as well as real-world data from four ICU patients. Although the results of their study showed that the CGO could be suppressed, several limitations of their proposed technique arise. First, the impulse responses used to estimate the CGO assume linearity and stationarity of the beating heart, which are not consistent with our understanding of physiological processes. And secondly, the technique requires at least one-minute worth of ECG data and 10 stable breathing efforts in order to "learn" and adapt to the patient's heart rate, which suggests that the technique is not suitable for short-term duration signals. 
In [42], a CGO suppression scheme using a modified adaptive noise cancellation (MANC) technique was proposed. The technique makes use of the conventional adaptive noise cancelling (ANC) structure using the least-mean squares (LMS) algorithm to adjust the filter coefficients. In ANC structures, two inputs are required: i) the input signal with undesirable characteristics, and ii) the reference signal. The output of the ANC structure is the error signal (i.e. the difference) between the input signal and the reference signal. In the case of [42], the input signal was the CGOcontaminated Peso signal, and the reference signal was selected as the airflow, which had similar temporal and spectral properties of Peso. The aforementioned selection of input and reference signals resulted in an error signal representative of the CGO. The proposed MANC structure subtracted the error signal of ANC (i.e. the CGO) from the original CGO-contaminated Peso to provide a "clean" version of the Peso. The performance of the MANC technique was tested on two Brown-Norway rats and shown to significantly improve the calculation of airway resistance, which is a respiratory system parameter. A major limitation of this study is the fact that the technique was tested solely on two Brown-Norway rats. Because the animal model exhibits different cardiopulmonary mechanics compared to humans in the ICU, it is difficult to infer the performance of the proposed MANC technique.

In [46], the method proposed in [45] was modified and applied in suppressing CGO from impedance pneumography (IP) signals that are used to assess lung function. Like in [45], an estimate of the CGO was generated using the R-waves from a corresponding ECG signal and subtracted from the IP signal. In this approach, the CGO estimation was modified to take into account lung volume induced changes. The method was found to attenuate CGO with minimal distortions to the respiratory portion of the IP signal. 
More recently, a template subtraction technique has been proposed by [43]. Drawing on work based on removing ECG contamination from EMG signals, the proposed technique aims to remove CGO from Peso using three steps. In the first step, the R-peak artifact in an EMG signal obtained at the costal margin (lower edge of the thorax located between the $7^{\text {th }}$ and $10^{\text {th }}$ ribs) is

detected. The fundamental idea behind this concept is that the locations of the R-peaks correspond to the location of the CGO in the Peso. The second step in the proposed technique is the creation of the artifact template, which is generated by taking an ensemble average of successive Peso segments separated by the R-peaks and normalized in time to unit length. In the final step, the length of the artifact template is scaled back to the length of each artifact and subtracted from the raw Peso signal. The proposed template-subtraction technique was tested on six mechanically ventilated patients receiving Pressure Support Ventilation (PSV), and has shown to be able to suppress CGO quite well. Furthermore, the template generated through this method resembles the filling pressures of the left atrium of the heart, which may potentially be useful in assessing the structure and function of the heart.

\subsection{Chapter Summary}

This chapter has described the anatomical and physiological background of breathing. Parameters such as $\mathrm{P}_{\mathrm{L}}$ and Peso were then introduced and their importance in guiding lungprotective mechanical ventilation strategies as well as managing treatments in critically-ill patients was summarized. Despite the technical limitations of using the Peso signal, its potential for clinical use has garnered a lot of interest in recent years. The rest of this chapter was devoted to describing how the Peso signal is acquired in the clinical context. From a biomedical signal processing perspective, the Peso signal's time-domain and frequency-domain representations were described in detail. This chapter concluded by introducing the CGO as a major source of interference 
encountered in the Peso signal, and a summary of the significant signal processing contributions developed for suppressing CGO was provided. The context provided in this chapter serves as motivation for the work presented in this thesis to develop new signal quality enhancement techniques to suppress CGO from Peso. The next chapter introduces the proposed enhancement scheme and its development based on proof-of-concept results. 


\section{Chapter 3}

\section{The Proposed Enhancement Scheme: Characterizing the Peso and CGO Signals, Preliminary Experimentation \& Proof-of- Concept}

\subsection{Motivation}

Suppressing cardiogenic oscillations (CGOs) in the Peso signal is undoubtedly an important task, because CGO interference can pose problems for respiratory monitoring in ICUs. Although some potential solutions have been developed, which was described in the previous chapter, many of those techniques required a reference signal such as an ECG or EMG. Although multimodal approaches may provide additional context to how one organ system affects another, simultaneous acquisition of those biomedical signals requires multiple systems and devices. Introducing additional systems and devices may lead to other challenges, which include ensuring patient comfort as well as data synchronization. To avoid these issues altogether, an adaptive, datadriven approach using solely the information from the Peso signal is proposed. The Peso signal and the CGO will be characterized using the decomposition algorithms, and the enhancement scheme will be developed and refined in this Chapter. 


\subsection{Proposed Enhancement Scheme}

\subsubsection{Overview}

\section{Empirical Mode Decomposition (EMD)}

The EMD algorithm is a popular data analysis method proposed by Huang in the late 1990s [47]. Over the past two decades, the algorithm has been applied in various research areas to adaptively decompose time series data into a finite set of zero-mean AM-FM components [48]. What makes the algorithm popular among researchers is the fact that EMD performs the decomposition without assuming linearity and stationarity of the data [47], [48]. Furthermore, the algorithm is entirely data driven; unlike tools such as the Fourier and Wavelet transforms that rely on basis functions (i.e. sinusoids and mother wavelets, respectively), EMD decomposes the time series based on intrinsic properties of the data [47]. The extracted AM-FM components described previously are known as intrinsic mode functions (IMFs), which represent the oscillatory modes embedded in the data [47], [48]. While the IMFs allow for the calculation of instantaneous frequency via the Hilbert Transform [47], [49], it has been suggested that the IMFs are also related to specific physical phenomena present in the measured data [49]-[50]. Since EMD is capable of decomposing nonlinear and non-stationary signals into components that can represent physical phenomena, the algorithm has been used successfully in many applications related to biomedical signal processing such as ECG enhancement and QRS detection [51], EEG artifact removal [52][53], tissue artifact removal from respiration signals [54], feature extraction for epilepsy detection [55] and more recently, to identify a child's autism severity level [56]. According to [47], the extracted components must satisfy two conditions to be considered an IMF:

1. The number of extrema and the number of zero crossings may only differ by one. 
2. The mean value between the envelopes generated by the local maxima and local minima must be zero at all times.

The IMFs are obtained by performing EMD on a given signal, $x(t)$, through the sifting process [47]. The EMD algorithm and the sifting process are described as follows [47], [48]:

1. Identify all local extrema in $x(t)$.

2. Generate the upper envelope, $e_{\text {upper }}(t)$, and lower envelope, $e_{\text {lower }}(t)$, using cubic spline interpolation from the maxima and minima, respectively.

3. Calculate the point-by-point mean between the upper and lower envelopes through EQ3.1:

$$
m(t)=\frac{e_{\text {upper }}(t)-e_{\text {lower }}(t)}{2}
$$

4. Subtract $m(t)$ from $x(t)$ to obtain a potential IMF, $h(t)$ :

$$
h(t)=x(t)-m(t)
$$

5. Check the properties of $h(t)$ :

a. If $h(t)$ satisfies the conditions to be considered an IMF, calculate the residual via EQ3.3:

$$
r(t)=x(t)-h(t)
$$

b. Otherwise, replace $x(t)$ with $h(t)$ and iterate through Steps 1 to 5 until $h(t)$ satisfies 5.a.

6. Repeat Steps 1 to 6 until the residual, $r(t)$, satisfies a predefined stopping criterion. The original time series signal, $x(t)$, can then be represented as a sum of the IMFs, $h_{i}(t)$ for $i=1 . . N$, and the residual, $r(t) \cdot x(t)$ can be represented mathematically through EQ3.4:

$$
x(t)=\sum_{i=1}^{N} h_{i}(t)+r(t)
$$


Based on the steps of the EMD algorithm, the highest frequency components are sifted first. Thus, the first IMF contains the highest frequency component of $x(t)$. The IMFs that follow progressively contains lower frequency components. The last IMF has the lowest frequency component present in the signal and represents the residual.

\section{Ensemble EMD (EEMD)}

Although EMD has been quite successful in many fields, a major obstacle associated with the algorithm is the concept of mode-mixing [57]. Mode-mixing occurs when a single IMF contains more than one oscillatory mode, which results in a loss of physical meaning for the IMF [50], [57]. To overcome the mode-mixing problem, Wu and Huang [57] developed a noise-assisted data analysis method known as Ensemble EMD (EEMD). The EEMD method decomposes a signal into its IMFs via EMD over several trials [57]-[58]. White noise with finite amplitude is added to the original signal at the start of each trial before proceeding with EMD, which results in an ensemble for each IMF [50], [57]. The ensemble average for each of the corresponding IMFs are taken, which are then treated as the true IMFs [19]. The addition of white noise during the process ensures that no scales are missing, thereby overcoming the problem of mode mixing [57]-[58]. Theoretically, the true IMFs do not contain the added white noise as they are cancelled out during the ensemble averaging [50], [57]. A flowchart describing how the IMFs are generated using the EEMD algorithm is shown in Figure 3.1. 
Initialize:

- Number of trials, $M$

- Amplitude of white noise

- Trial counter, $m=1$

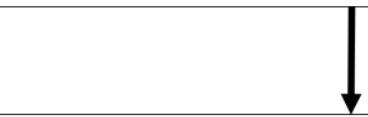

For the $m^{\text {th }}$ trial, generate $\mathrm{x}_{\mathrm{m}}(\mathrm{t})$ by adding white noise, $n_{m}(t)$, to the original signal, $x(t)$ :

$$
x_{m}(t)=x(t)+n_{m}(t)
$$
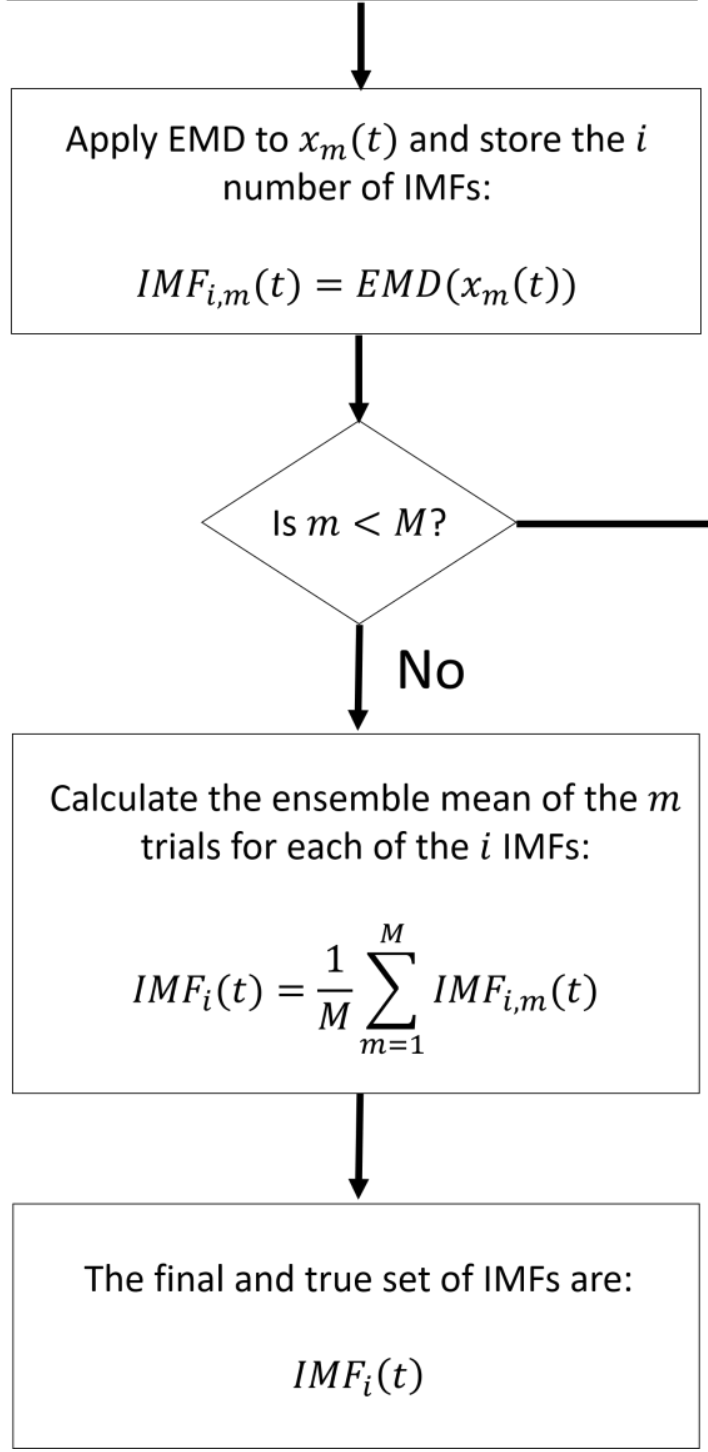

Figure 3.1. A flowchart representing how EEMD generates the IMFs of a given signal. 


\subsubsection{Enhancement Scheme}

As mentioned previously, the main form of interference observed in Peso signals are cardiogenic oscillations (CGOs) caused by the beating heart. While various pressures acting on the esophageal balloon may influence the measured signal, $\mathrm{P}_{\text {meas }}$, the work presented in this thesis assumes that $\mathrm{P}_{\text {meas }}$ contains only two components superimposed on each other: i) the esophageal pressure (Peso) and ii) the CGO. The assumption of the measured signal is described by EQ3.5:

$$
P_{\text {meas }}=P_{\text {eso }}+C G O \quad(\text { EQ3. 5) }
$$

To suppress the cardiogenic oscillations from the measured signal, the decomposition algorithms described in the previous section were used to decompose $\mathrm{P}_{\text {meas }}$ into its IMFs. The IMFs relevant only to Peso would be selected and used to reconstruct $\mathrm{P}_{\text {meas }}$ such that CGO is no longer a component in the signal. Thus, the goal of this work is to apply the decomposition algorithms to $\mathrm{P}_{\text {meas }}$ and achieve the following relation:

$$
P_{\text {meas,enhanced }}=P_{\text {eso }} \quad(\mathbf{E Q 3 . 6 )}
$$

Based on the frequency bandwidths described in Section 3.2.2, the components related to CGO are expected to appear in the first few IMFs while the Peso components should appear in higher valued IMFs since the EMD algorithm sifts the signal in the order of highest frequency to lowest frequency. The reconstruction of the enhanced version of $\mathrm{P}_{\text {meas }}\left(\mathrm{P}_{\text {meas,enhanced }}\right)$ is described in EQ3.7 in which the IMFs related to Peso (IMFPeso) are summed together.

$$
P_{\text {meas,enhanced }}=\sum I M F_{\text {Peso }}
$$


A general visual representation of the proposed enhancement scheme is shown in Figure 3.2. The complete methodology of this collaborative work between Ryerson University and St. Michael's Hospital, from signal acquisition to signal processing, is shown in Figure 3.3.

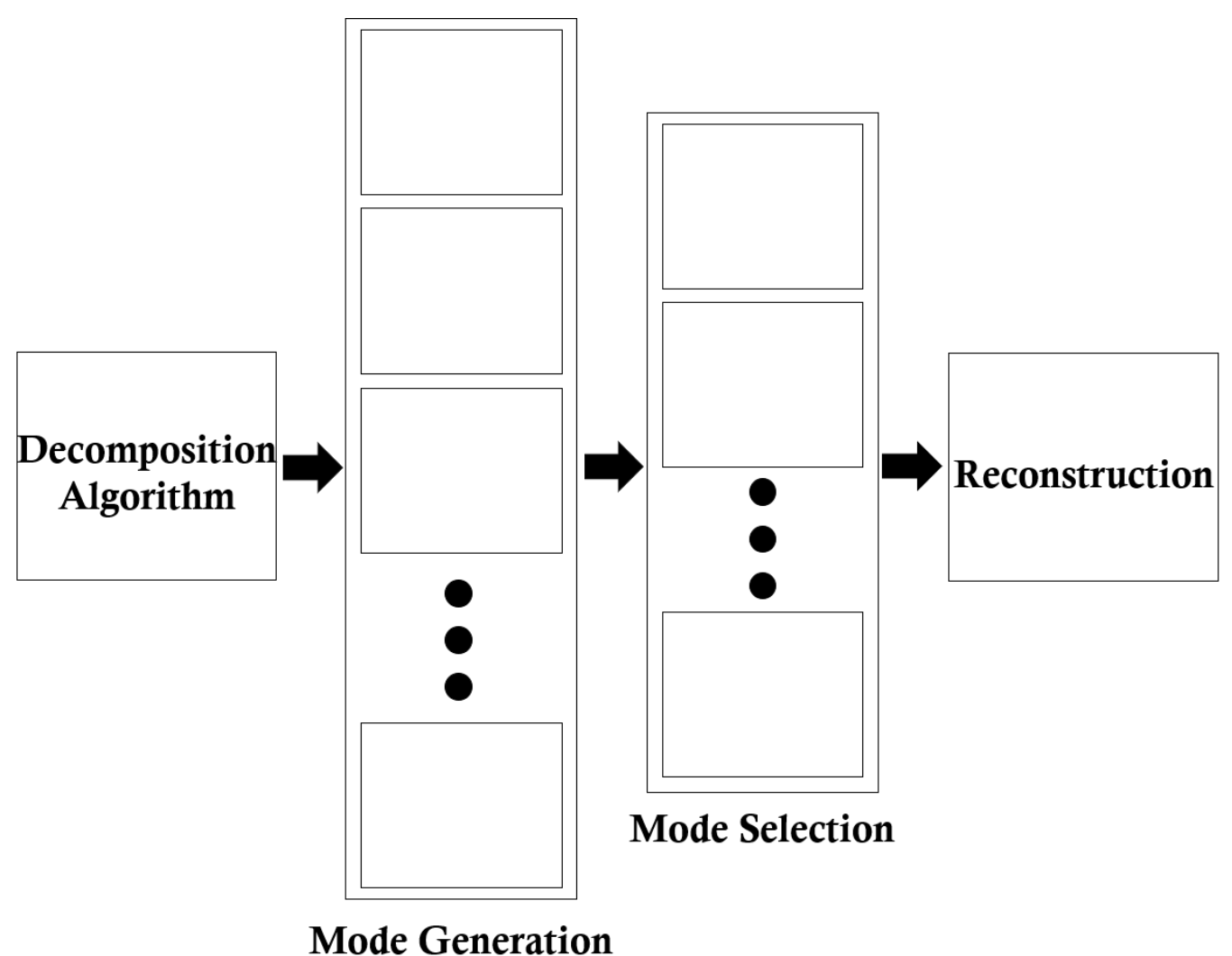

Figure 3.2. The workflow of the proposed enhancement scheme. The raw ("noisy") signal will first be decomposed using a decomposition algorithm (i.e. EMD or EEMD) to generate the IMFs. The IMFs relevant to the physiological process being measured are then selected. The IMFs are summed together to reconstruct a "clean" signal that is free from noisy/interference seen in the original raw signal. 

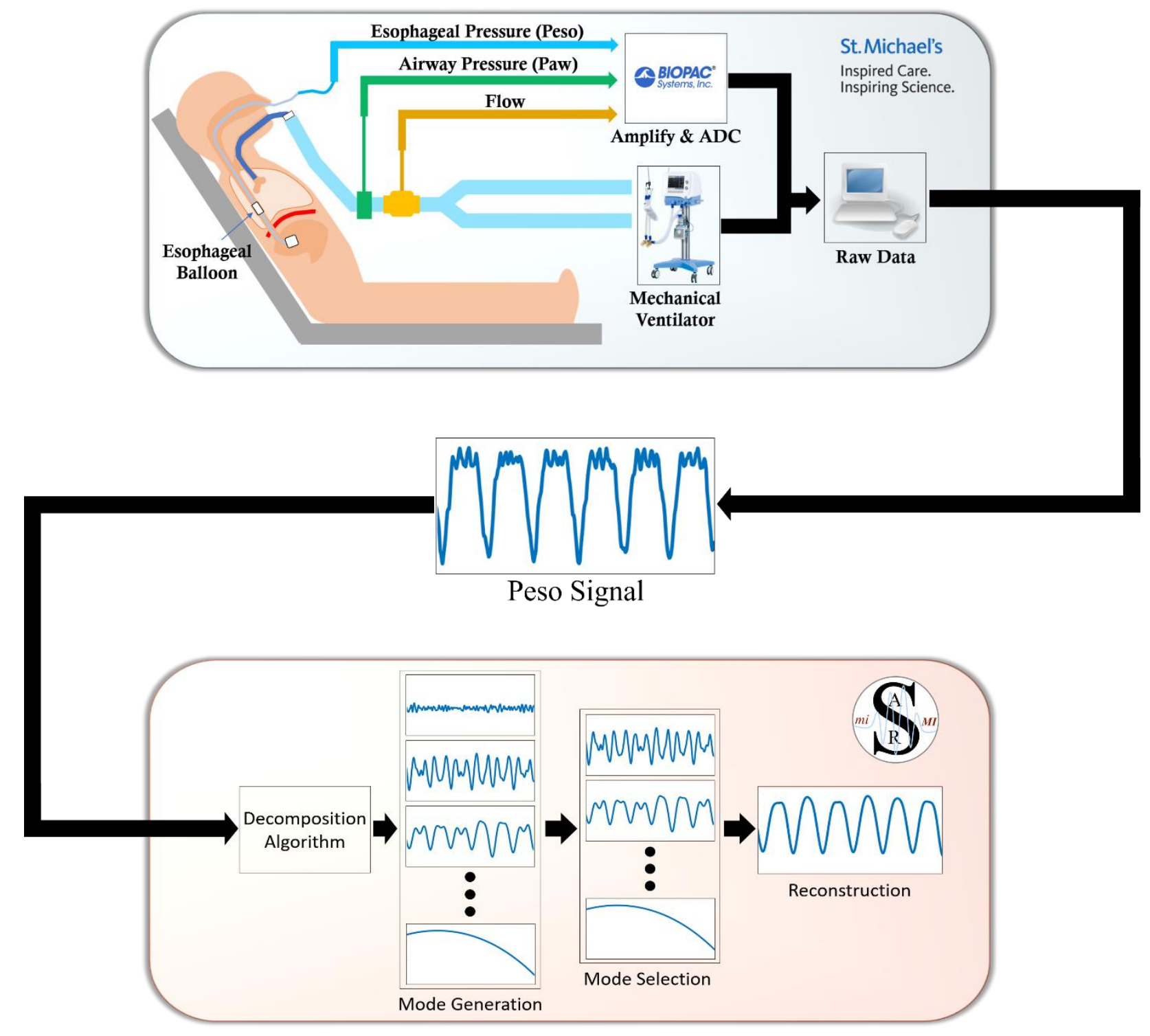

Figure 3.3. The complete methodology of this research work. This is a collaborative project between Ryerson University and St. Michael's Hospital under the Institute of Biomedical Engineering, Science, and Technology (iBEST). The raw Peso signal is acquired using an esophageal catheter by clinicians at St. Michael's Hospital, as shown in the blue box. The raw Peso signal is then provided to the Signal Analysis Research (SAR) Lab at Ryerson University, where the proposed enhancement technique (shown in the pink box) is used to remove/suppress the CGO interference from the Peso signal. 


\subsection{Characterizing the CGO Interference and Proof-of-Concept of Proposed}

\section{Enhancement Scheme}

The assumption made about the raw Peso signal ( $\left.\mathrm{P}_{\text {meas }}\right)$, as described in EQ3.5, is that it is comprised of two major components: i) the esophageal pressure (Peso) and ii) the cardiogenic oscillations (CGO). To get an understanding of the two components of $\mathrm{P}_{\text {meas, }}$, data from patients in "passive breathing" conditions were used. This Section is focused on gaining insights into the characteristics of CGOs. Additionally, the enhancement scheme proposed in the previous section will be applied to generate a proof-of-concept to assess the feasibility of the EMD algorithm to suppress interference from Peso signals.

\subsubsection{Dataset}

The first dataset was used to characterize the CGO, which represents the major source of interference that the work in this thesis is trying to remove/suppress. This dataset will also be used to generate proof-of-concept results of the proposed enhancement scheme. There are five types of data in this dataset: i) Time [seconds], ii) Flow [L/s], iii) Airway Pressure [ $\left.\mathrm{cmH}_{2} \mathrm{O}\right]$, iv) Esophageal Pressure $\left[\mathrm{cmH}_{2} \mathrm{O}\right]$, and v) Transpulmonary Pressure $\left[\mathrm{cmH}_{2} \mathrm{O}\right]$. The data was sampled at $25 \mathrm{~Hz}$ and was collected from two passively breathing patients receiving mechanical ventilation support. In the context of critical care medicine, the "passive breathing" condition means that the patient does not provide any effort in taking a breath; it is solely the mechanical ventilator that performs the role of respiration for the patient. To achieve the passive breathing condition in this dataset, the patients were sedated, and a paralysis-inducing drug was administered to their respiratory muscles. A low-flow inflation maneuver was performed on the patients where the ventilator inflated their lungs at a constant flow rate of $5 \mathrm{~L} / \mathrm{min}$. The duration of the two signals are approximately 20 
seconds and 25 seconds. This dataset was approved by the St. Michael's Hospital's Research Ethics Board under REB\# 15-074. The dataset used in this Section is summarized in Table 1.

TABLE 1: SUMMARY OF THE DATASET USED FOR GETTING A BASELINE UNDERSTANDING OF CGOS.

\begin{tabular}{|c|c|c|c|c|c|c|}
\hline $\begin{array}{c}\text { Dataset } \\
\#\end{array}$ & REB \# & $\begin{array}{c}\text { Size of } \\
\text { Dataset } \\
\text { (\# of } \\
\text { patients) }\end{array}$ & of Patients & Types of data & $\begin{array}{c}\text { Sampling } \\
\text { Frequency } \\
\text { (Hz) }\end{array}$ & $\begin{array}{c}\text { Duration } \\
\text { of Signals } \\
\text { (sec) }\end{array}$ \\
\hline 1 & $15-074$ & 2 & Passive & $\begin{array}{l}\text { 1. Time } \\
\text { 2. } \begin{array}{l}\text { Flow } \\
\text { 3. Airway Pressure } \\
\text { 4. Esophageal } \\
\text { Pressure } \\
\text { Transpulmonary } \\
\text { Pressure }\end{array}\end{array}$ & 25 & $20-\sim 25$ \\
\hline
\end{tabular}

3.3.2 Experimental Setup and Validation - Characterizing CGOs \& Proof-of-Concept Results

The specific conditions behind data acquisition, as described in the previous section, are important to note for the experimental setup covered in this section. Since paralysis-inducing drugs were administered to the patients' respiratory muscles, the patients were unable to provide any form of respiratory effort. As a result, only the activity of the heart in the form of CGOs, as well as the low-flow inflation maneuver are observed in the Peso signal.

To gain insights on the proof-of-concept results and determine the feasibility of the proposed enhancement scheme, our clinical collaborators were asked to provide their opinions as domain-experts. This form of validation is known as "Face Validity," which relies on the subjective assessment of an expert about the appearance of a specific measurement or variable. In this work, the specific measurements used for face validity are the IMFs, the quality of the extracted CGO in terms of its morphology, and the quality of the enhanced Peso signal in terms of its morphology and the slope of the Peso deflection $\left(\frac{d \text { Peso }}{d t}\right)$. 


\subsubsection{Results and Discussions}

\section{Characterization of CGO}

As mentioned in the previous Chapter, a basic understanding of a signal's characteristics can be derived from the time-domain representation of the signal. Figure 3.4 (left) illustrates the time-domain representation of the Peso signal. The low-flow inflation maneuver is represented by a linear increase in pressure starting at $\sim 7$ seconds and ending at 12 seconds. The duration of the low-flow inflation maneuver is when inspiration occurs. Upon reaching the maximum value, the pressure declines at a steep rate from 12 seconds until it reaches a baseline value starting at $\sim 13.5$ seconds. The declining pressure represents the expiration process. In addition to the low-flow inflation maneuver, another strikingly clear characteristic of the Peso signal for this patient are the low amplitude pressure variations exhibiting quasi-periodic behaviour. The oscillatory pressure fluctuations are the main form of interference in the Peso signal: the CGOs. It is important to note that the DC value of the Peso in Figure 3.4 has not been removed; this is by recommendation of the collaborating clinicians as there is no clinical reason to remove it.

Because the CGOs are evidently quasi-periodic in nature, the frequency-domain may also be helpful in providing further insights into the signal. The frequency-domain representation of the Peso signal described previously is shown in the magnitude spectrum of Figure 3.4 (right). The large peak indicated by the blue arrow is the fundamental frequency of the CGO for the specific patient at the time of data acquisition. The smaller magnitude peaks indicated by green arrows represent the harmonics of the CGO. It is important to note that there is one more peak that occurs at a lower frequency than the CGO fundamental frequency; this peak represents the DC value as well as the magnitude of the frequency generated by the low-flow maneuver. 

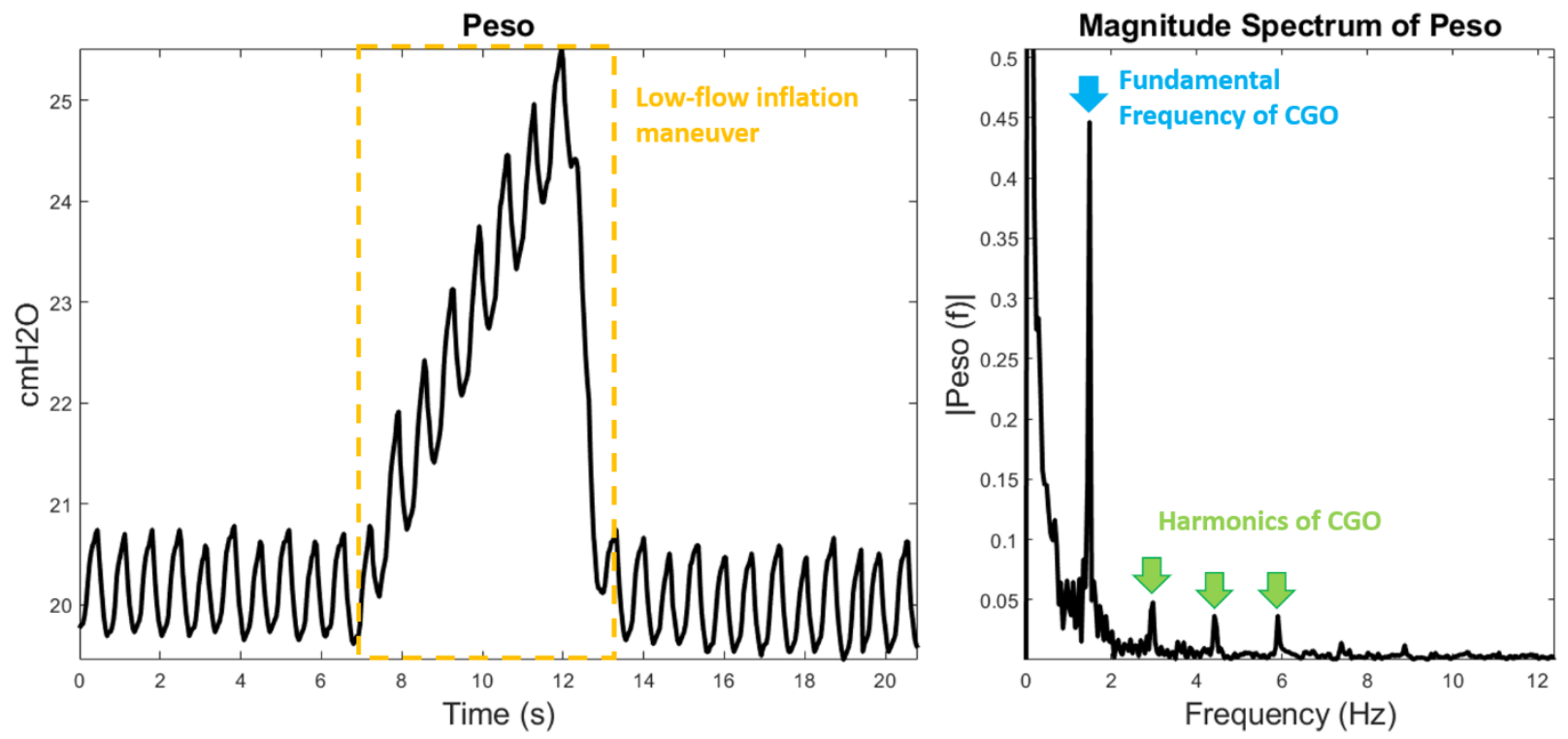

Figure 3.4. The Peso signal in time-domain on the left. The low-flow inflation maneuver manifests itself as a linear increase in pressure during inspiration and a steep decline in pressure during expiration, while the CGOs are represented by small oscillatory fluctuations throughout the timeseries. The magnitude spectrum of the Peso signal is shown on the right. The fundamental frequency of the CGO is indicated by the blue arrow, and the harmonics of the CGO are labelled with green arrows. 
It is important to note that although the characteristics of the CGO shown in this Section are consistent with the physiological understanding of the heart (i.e. the oscillatory fluctuations caused by the depolarization and repolarization of the heart chambers, as well as the harmonics of the heart beat seen in the magnitude spectrum), there is a small-dataset problem. Only two signals from two passively breathing patients were provided, and therefore not representative of the entire population. Nevertheless, gaining some insight into the CGO signal characteristics will be helpful in distinguishing between Peso and CGO.

\section{Application of Proposed Enhancement Scheme - A Proof-of-Concept}

It is evident from Figure 3.4 that the CGO is a major source of interference, which poses an issue for clinicians when trying to interpret their patients' respiratory mechanics. The EMD algorithm was applied to the Peso time-series signal shown in Figure 3.4 as a proof-of-concept to assess the feasibility of using the enhanced Peso for clinical use.

Applying EMD yielded nine IMFs, which are shown in Figure 3.5. Based on the bandwidths of the relevant physiological processes found in literature and the preliminary characterization of CGO in the previous section, it is expected that the CGO would appear in IMFs containing higher frequency bands (i.e. first few IMFs). On the other hand, the Peso, or in this case the low-flow inflation maneuver, would be expected to appear in the IMFs representing lower frequency bands (i.e. IMFs after CGO). 


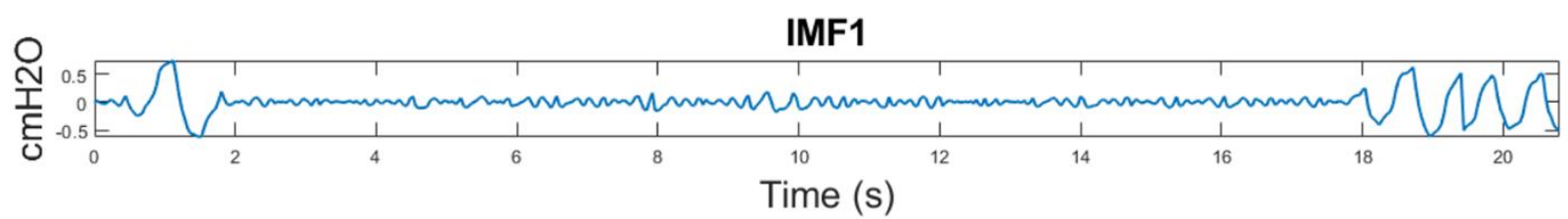

IMF2

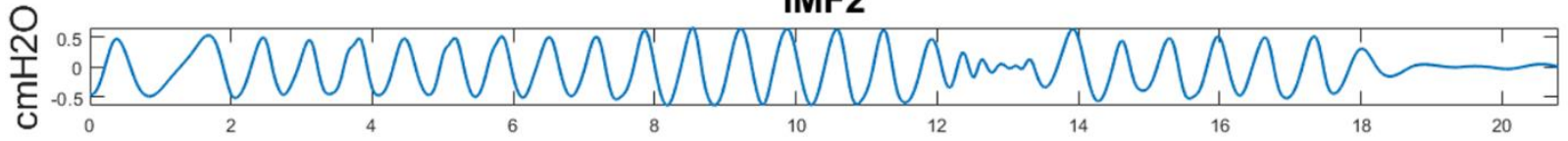

Time (s)

IMF3

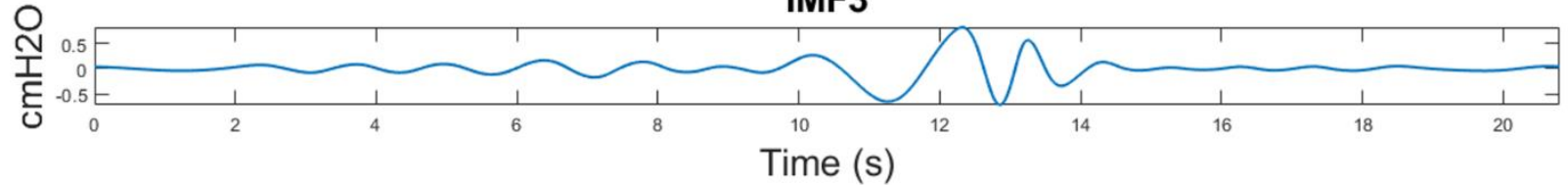

IMF4

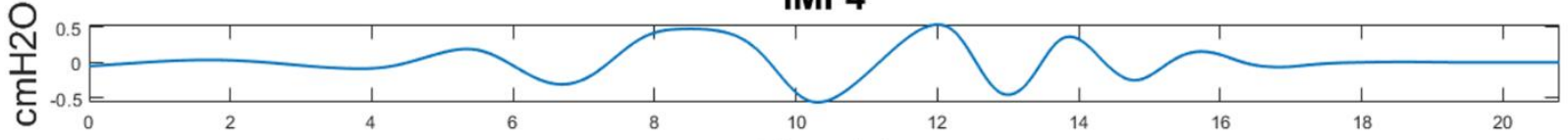

Time (s)

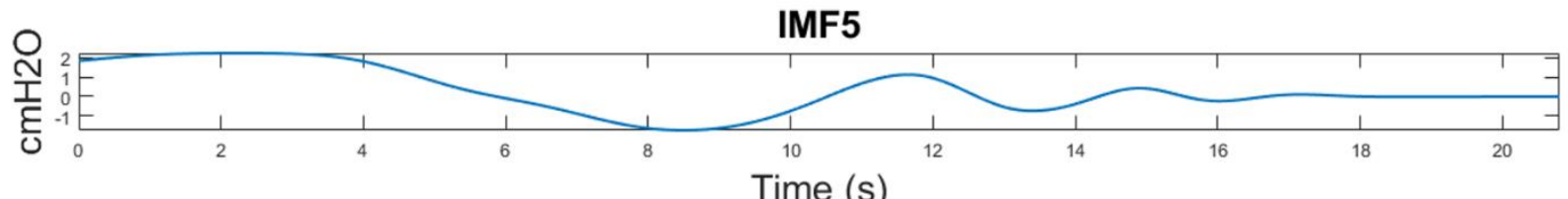

Time (s)

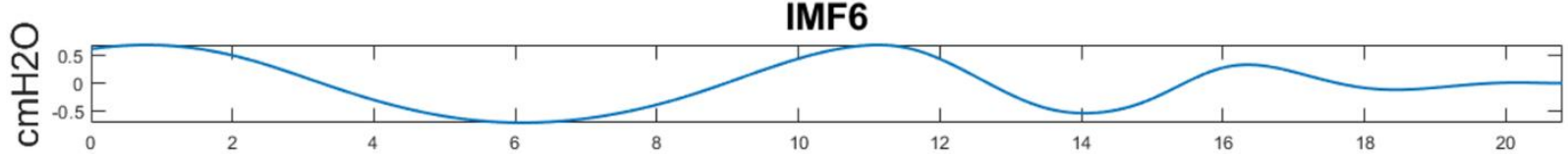

Time (s)

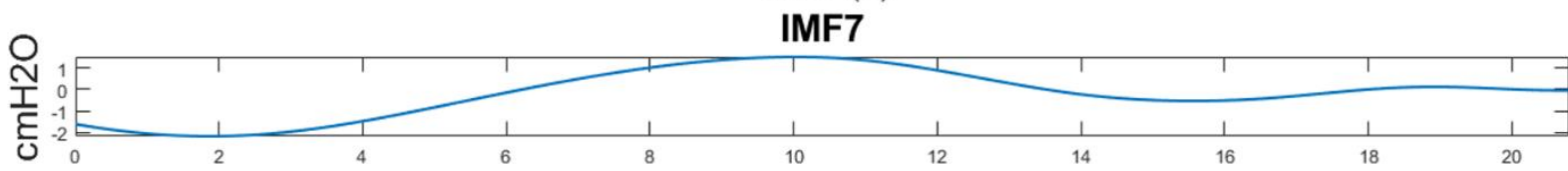

Time (s)

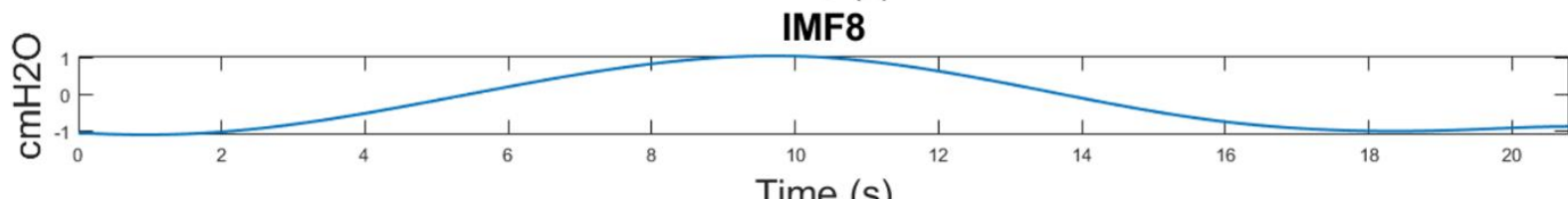

Time (s)

IMF9

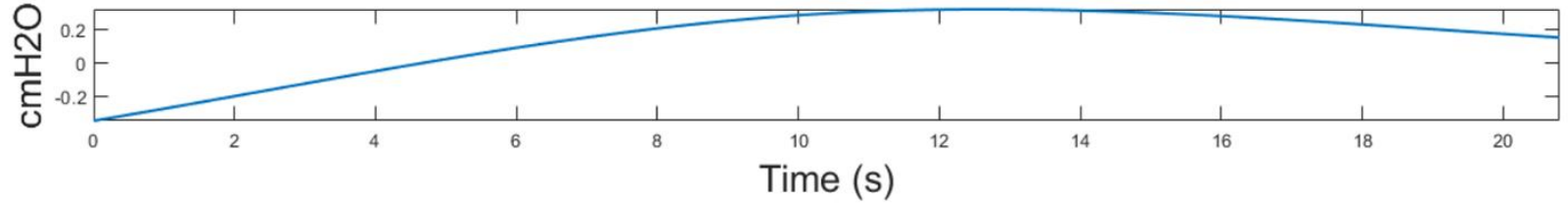

Figure 3.5. The IMFs of the Peso signal using EMD. 


\section{$\underline{\text { Extraction of CGOs }}$}

Through visual inspection of Figure 3.5 in conjunction with clinical collaborators, it appears that IMF1 and IMF2 certainly contain information about the CGO. Additionally, IMF3 may also contain CGO information. To test the claim, two CGO signals were reconstructed by selecting the following IMF combinations:

i) IMF1 and IMF2

ii) IMF1, IMF2, and IMF3.

The two CGO signals were plotted together along with the original Peso signal for comparison as seen in Figure 3.6 (A). There were two notable sections in Figure 3.6 (A) that provided information about the reconstruction using the above-mentioned claim. The first section, which is encapsulated by a yellow box, is a portion of the Peso signal that contains only the CGO. The second section is the portion of the Peso signal during the low-flow inflation maneuver.

The first section shown in the yellow box in Figure 3.6 (A) is shown more clearly in Figure 3.6 (B). Through a superficial assessment of the CGO signals in Figure 3.6 (B) by the clinical collaborators, the morphologies of the reconstructed CGOs (red and blue) were found to be quite similar to the morphology of the original CGO (black). Not only were the peak-to-peak amplitude values of the signals very similar, but the "landmarks" of the waveforms, such as the notches seen throughout the CGO signal, were also reconstructed. The IMFs used to reconstruct the CGOs in Figure 3.6 (B) suggest that there is very little difference between the IMF combinations described in (i) and (ii).

Based on the observations made from Figure 3.6 (B), the portion of the Peso signal representing the low-flow inflation maneuver shown in Figure 3.6 (C) may provide further insight 


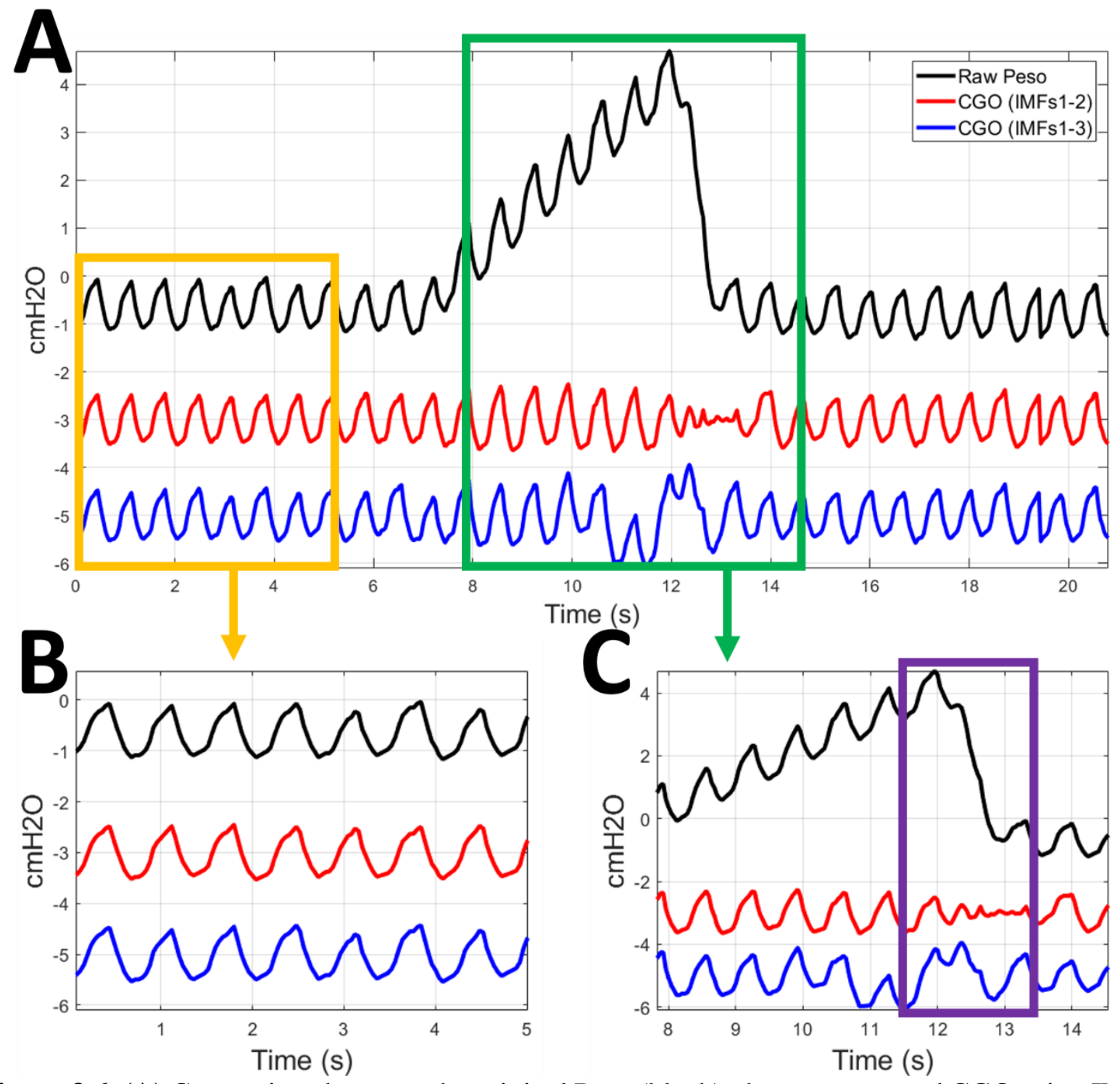

Figure 3.6. (A) Comparison between the original Peso (black), the reconstructed CGO using IMFs 1 to 2 (red), and the reconstructed CGO using IMFs 1 to 3 (blue). The DC value of the original Peso was removed, an artificial value of $-3 \mathrm{cmH}_{2} \mathrm{O}$ was added to the $\mathrm{CGO}$ using IMFs 1 to 2 (red), and a value of $-5 \mathrm{cmH}_{2} \mathrm{O}$ was added to the CGO using IMFs 1 to 3 (blue) for a clear comparison of the waveforms. (B) A portion of the original Peso signal containing only CGO. The section of the signal portrayed in this plot is encapsulated by the yellow box in (A). (C) A portion of the original Peso signal containing the low-flow maneuver, which is extracted from the green box in (A). The expiration process of the maneuver is shown in the purple box. 
into determining which IMFs truly represent the CGOs. During the inspiration phase of the maneuver (i.e. the portion of the Peso with a linearly increasing pressure over time), the CGO reconstructions are still quite similar to the original $\mathrm{CGO}$, albeit without the linear increase caused by inspiration. However, the morphologies of the CGOs appear to differ during the expiration phase (i.e. the steep, negative slope) indicated by the purple box of Figure 3.6 (C). The expiration phase appears to last approximately one second. Based on the frequency of the CGOs prior to expiration, it is suspected that at least one CGO cycle is present in the negative deflection but is hidden within the larger amplitude of the Peso signal itself. The red signal (reconstructed using IMFs 1 to 2) shows that a small component of the CGO is present during the expiration phase, while a larger CGO component is seen in the blue signal (IMFs 1 to 3). Through face validation, it was agreed that IMFs 1 to 3 most resembled the activity of the beating heart for this specific person.

\section{Extraction of Peso}

To extract the Peso signal represented by the low-flow inflation maneuver, the IMFs from Figure 3.5 were again shown to the clinical collaborators. It was suggested that the following IMF combinations may be used to reconstruct the Peso signal:
i) IMF3 to IMF9
ii) IMF4 to IMF9
iii) IMF5 to IMF9

The three combinations listed above were used to reconstruct three Peso signals. The three Peso signals were plotted separately alongside the original Peso signal and are illustrated in Figure 3.7. 

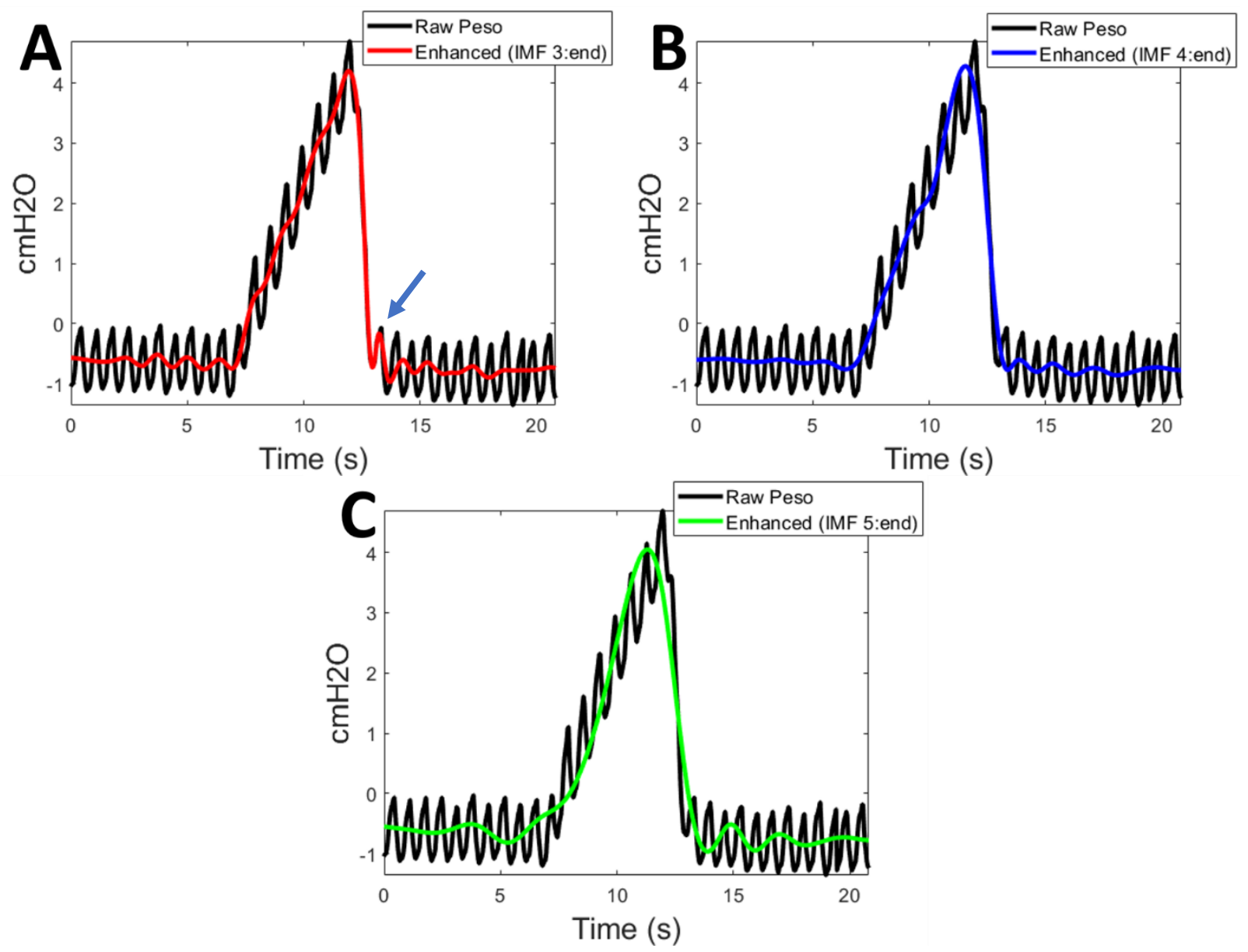

Figure 3.7. (A) The reconstructed Peso signal using IMFs 3 to 9 shown in red. The blue arrow indicates that a CGO cycle still persists after the application of the proposed signal enhancement scheme. (B) The reconstructed Peso signal using IMFs 4 to 9 shown in blue. (C) The reconstructed Peso signal using IMFs 5 to 9 shown in green.

In Figure 3.7 (A), IMF3 was selected as the starting IMF. The reconstructed Peso in red appears to follow the low-flow inflation maneuver quite closely. Although a significant portion of the fluctuations caused by the CGO have been removed, it is still apparent in the morphology that the CGO interference is still present. Furthermore, as indicated by the blue arrow, a CGO pulse persists at the end of the expiratory phase. Thus, the reconstruction of (i) IMF3 to IMF9 was deemed unsuitable for interference removal for this patient as it still contains significant CGO information. 
The next two combinations shown in Figure 3.7 (B) and Figure 3.7 (C) represent the Peso reconstructions using IMFs 4 to 9 and IMFs 5 to 9, respectively. For both signals, it was found that the CGO interference was suppressed relatively well while maintaining the shape of the low-flow inflation maneuver. However, Figure 3.7 (C) differs from Figure 3.7 (B) in that the reconstruction using IMFs 5 to 9 contains a larger delay in the pressure drop during expiration compared to the reconstruction using IMFs 4 to 9. According to the clinical collaborators, the slope of the Peso drop (i.e. $\frac{d P e s o}{d t}$ ) contains crucial clinical information. As a result, the reconstructed Peso using IMFs 5 to 9 was deemed unacceptable. Thus, the combination of IMFs 4 to 9 was selected as the acceptable IMFs for Peso extraction for this specific patient.

\section{Mode-Mixing of the Proposed Enhancement Scheme using EMD}

The proof-of-concept results presented in this section show that the proposed enhancement scheme using EMD is capable of not only removing the CGO interference from the Peso signal but is also able to extract the CGO signal itself. Despite the promising results, there is one significant issue that was observed in Figure 3.5: mode-mixing between IMF 1 and IMF 2. Figure 3.8 illustrates the mode-mixing problem seen in IMFs 1 and 2. The components in IMF 1 within the red squares should appear in IMF 2, which suggests that EMD may not be appropriate.

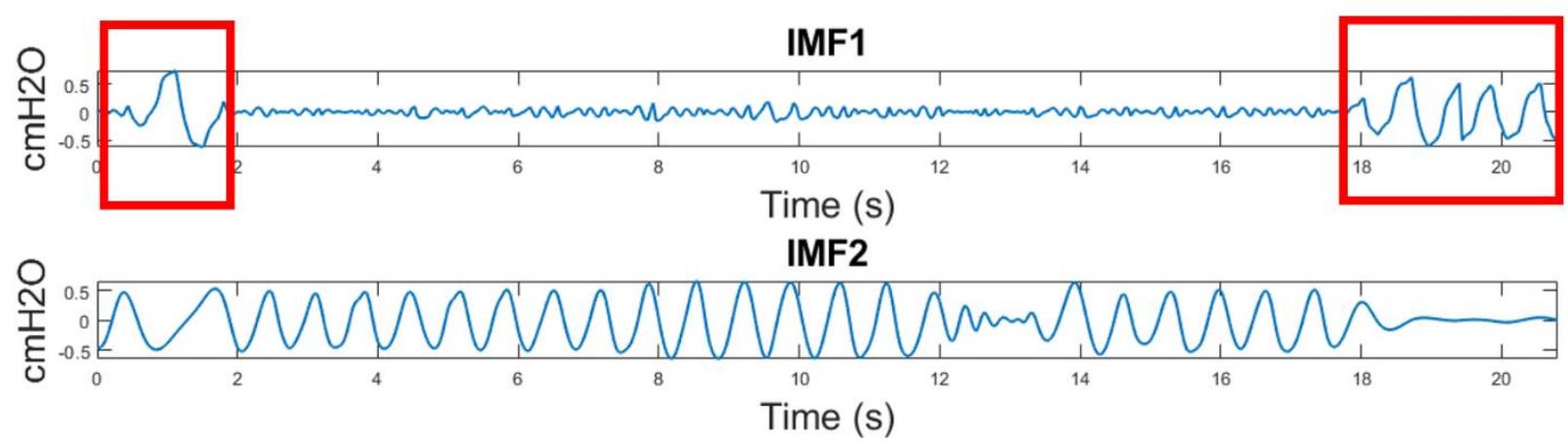

Figure 3.8. Mode-mixing problem observed in IMFs 1 and 2. The oscillatory components in IMF 1 that are shown in red should have appeared in IMF2. 


\subsection{Refining the Proposed Enhancement Scheme}

Section 3.3 showed that the proposed enhancement scheme is capable of not only suppressing the CGO interference, but also extracting the CGO signal itself. However, it was also found that the mode-mixing problem was present when the EMD algorithm was used. Because the mode-mixing problem renders the interpretation of IMFs physically meaningless, another decomposition algorithm must be used. The aim of this section is to refine the proposed enhancement scheme by applying Ensemble Empirical Mode Decomposition (EEMD) to determine if the mode-mixing problem would be solved.

\subsubsection{Dataset}

The dataset used in this section is the same data described in Section 3.3. A summary of the data used in this section is shown in Table 2.

TABLE 2: SUMMARY OF THE DATASET USED FOR REFINING THE PROPOSED ENHANCEMENT SCHEME.

\begin{tabular}{|c|c|c|c|c|c|c|}
\hline $\begin{array}{c}\text { Dataset } \\
\#\end{array}$ & REB \# & $\begin{array}{c}\text { Size of } \\
\text { Dataset } \\
\text { (\# of } \\
\text { patients) }\end{array}$ & $\begin{array}{c}\text { Condition } \\
\text { of Patients }\end{array}$ & Types of data & $\begin{array}{c}\text { Sampling } \\
\text { Frequency } \\
\text { (Hz) }\end{array}$ & $\begin{array}{c}\text { Duration } \\
\text { of Signals } \\
\text { (sec) }\end{array}$ \\
\hline 1 & $15-074$ & 2 & Passive & $\begin{array}{l}\text { 1. Time } \\
\text { 2. Flow } \\
\text { 3. Airway Pressure } \\
\text { 4. Esophageal } \\
\text { Pressure } \\
\text { Transpulmonary } \\
\text { Pressure }\end{array}$ & 25 & $20-\sim 25$ \\
\hline
\end{tabular}

3.4.2 Experimental Setup - Refining the Proposed Enhancement Scheme

The EMD and EEMD algorithms will be applied to the two sets of data described in Section

3.4.1 to make a comparison between the IMFs generated by both algorithms. For the EEMD 
algorithm, the number of ensemble trials used in the decomposition was fixed at 100 to maintain a sufficiently good SNR while reducing computational complexity [58]. The amplitude of white noise added into each trial was computed using EQ3.8 according to [57]:

$$
n=0.2 \times \operatorname{std}(\text { Peso }) \quad(\text { EQ3.8) }
$$

The methods used in Section 3.3 for the extraction of the CGO and enhancement of Peso were repeated.

\subsubsection{Results and Discussion}

A comparison of the IMFs generated using EMD and EEMD for the passively breathing patient described in Section 3.3 are shown in Figure 3.9 and Figure 3.10, respectively. Application of both decomposition algorithms yielded nine different IMFs. However, as noted previously in Section 3.3, the mode-mixing problem caused by EMD is clearly shown in IMFs 1 and 2 of Figure 3.9. By applying EEMD, it appears that the mode-mixing problem has largely been overcome as seen in Figure 3.10.

Because the IMFs generated using EEMD were different, it was expected that the enhanced Peso signal as well as the extracted CGO signals obtained using EMD's IMFs would also change. The same methods in Section 3.3 were applied for CGO extraction and Peso enhancement, but this time using EEMD's IMFs. Table 3 summarizes the relevant IMFs used to reconstruct the CGO and Peso signals for both EMD and EEMD. A visual comparison of the reconstructed CGO using EMD and EEMD is shown in Figure 3.11(A), and likewise, the reconstructed Peso is shown in Figure 3.11(B). 


\section{IMF1}

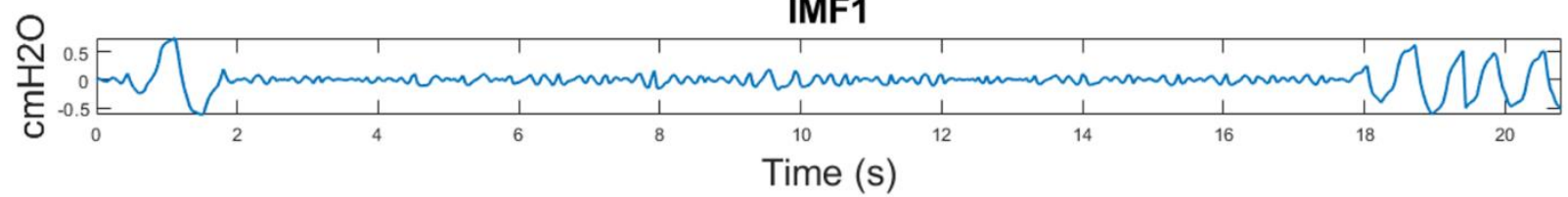

IMF2

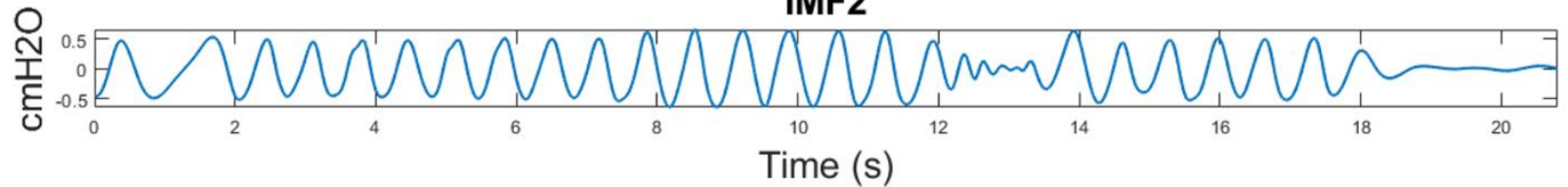

IMF3

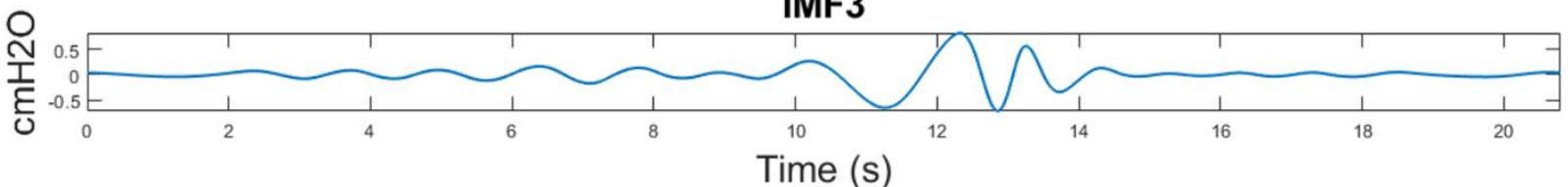

IMF4

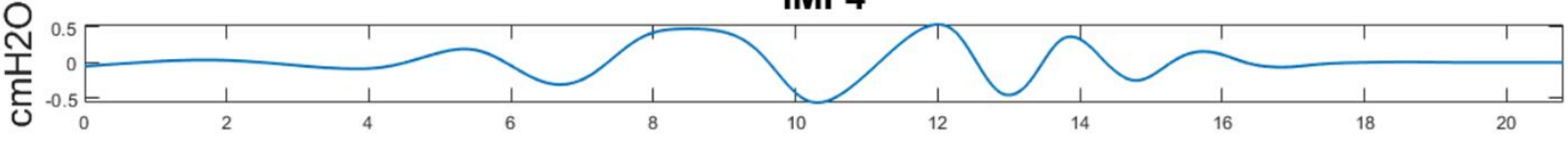

Time (s)

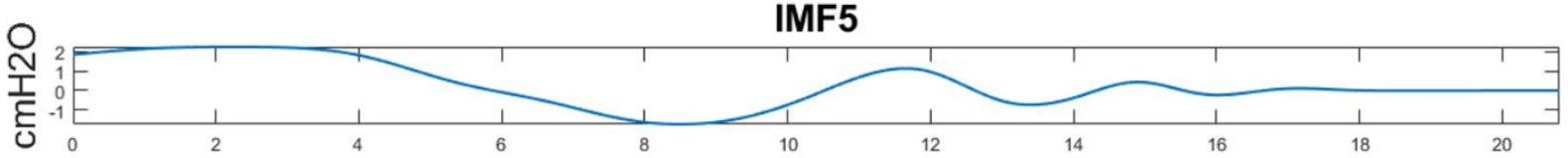

Time (s)

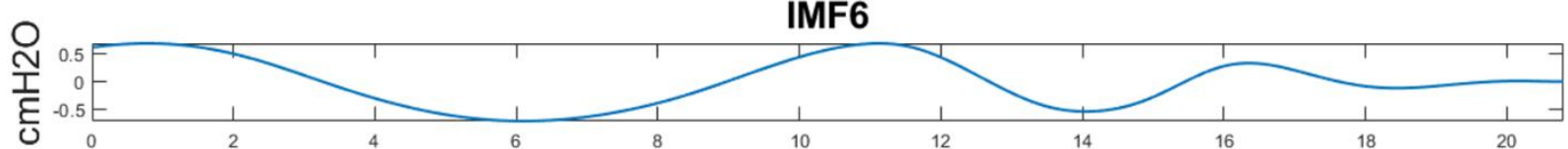

Time (s)

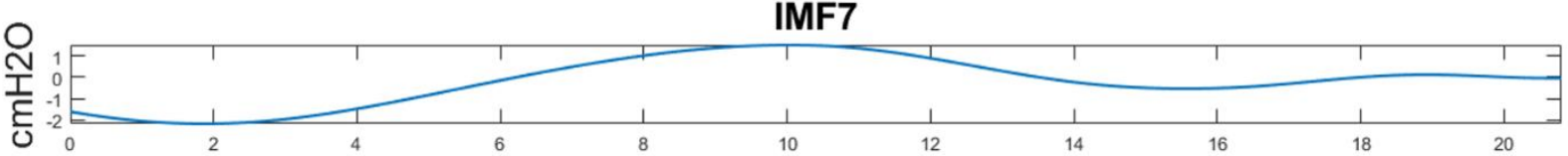

Time (s)

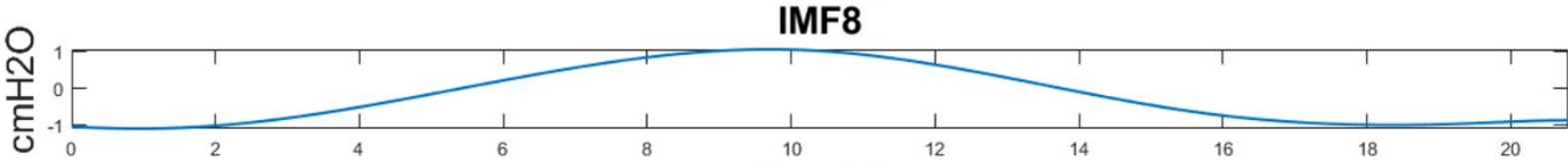

Time (s)

IMF9

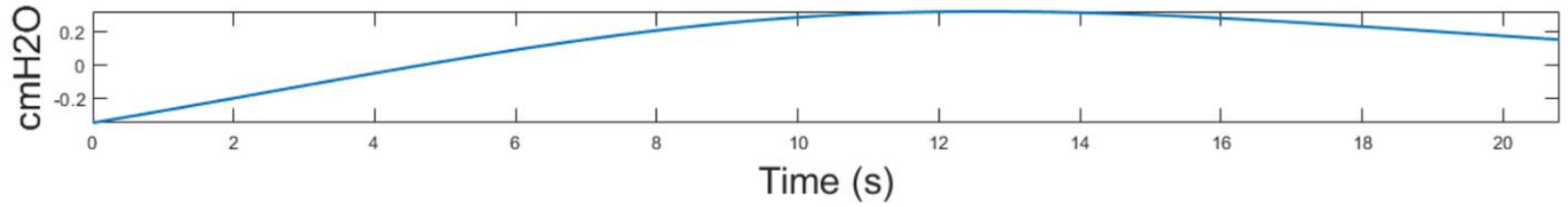

Figure 3.9. The IMFs generated using EMD for the passively breathing patient (recreation of Figure 3.5). 

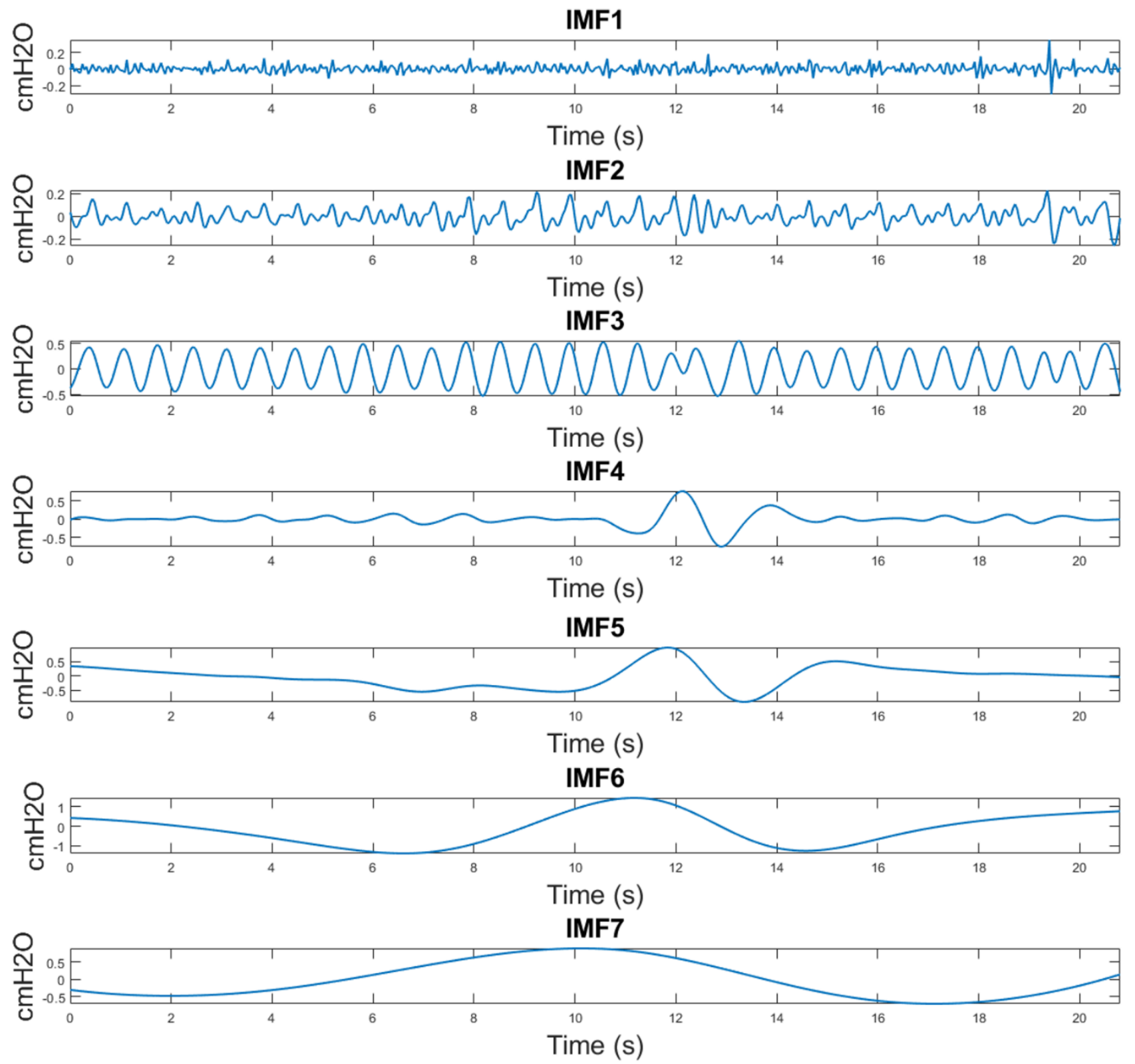

IMF8

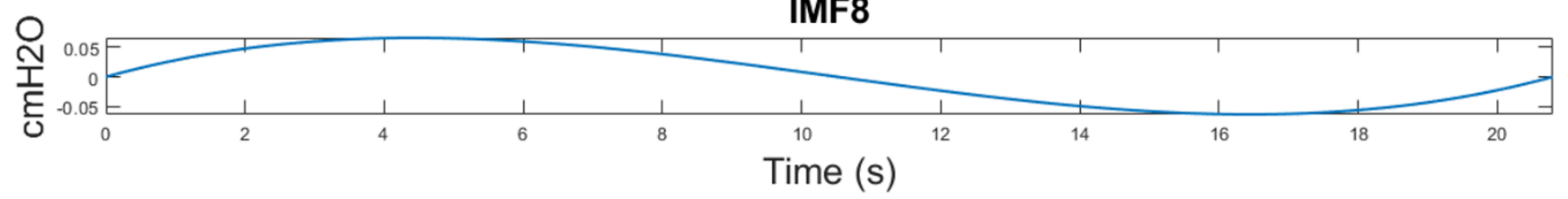

IMF9

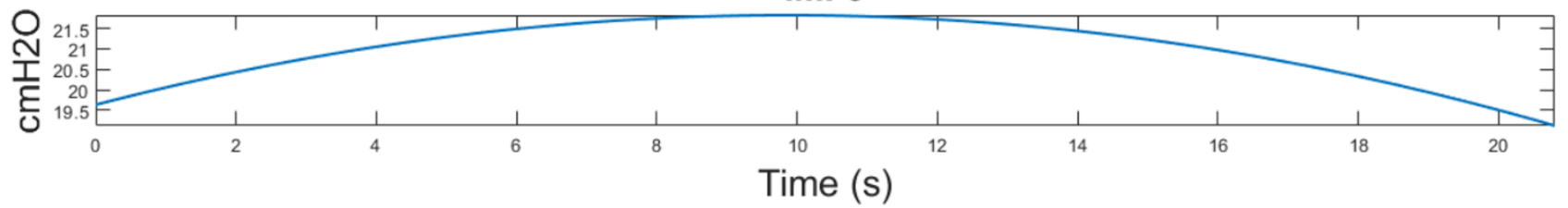

Figure 3.10. The IMFs generated using EEMD for the same passively breathing patient seen in Figure 3.9. 

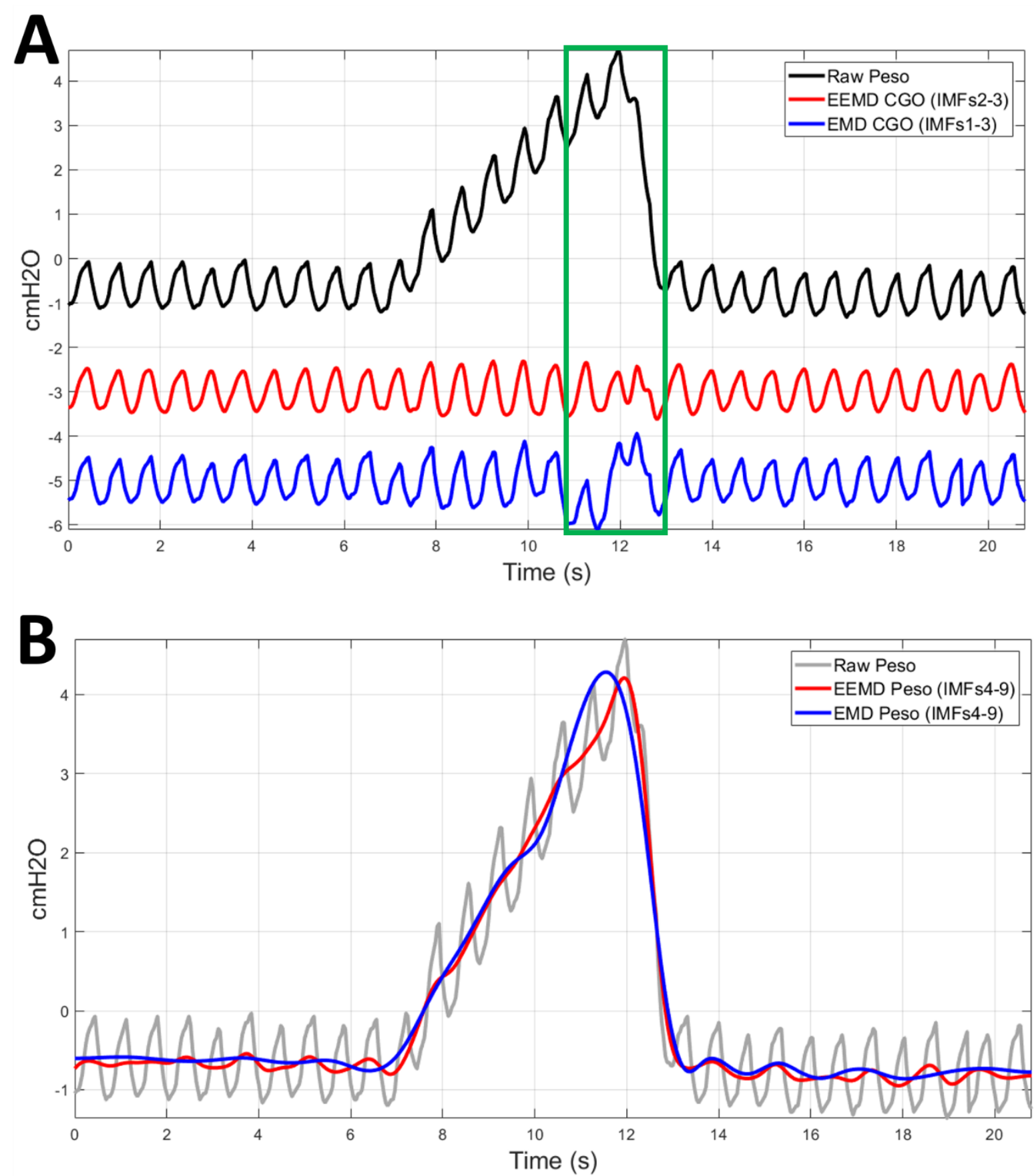

Figure 3.11. (A) The original raw Peso (black) with the DC value removed, the extracted CGOs using and EEMD's IMF 2 to IMF 3 (red) with an added value of $-3 \mathrm{cmH}_{2} \mathrm{O}$, and EMD's IMF 1 to IMF 3 (blue) with an added $-5 \mathrm{cmH}_{2} \mathrm{O}$. (B) The enhanced Peso using EEMD's IMF 4 to IMF 9 (red) and EMD's IMF 4 to IMF 9 (blue) superimposed on the original raw Peso (grey). 
TABle 3: The IMFs used FOR CGO EXTRACTION AND Peso ENHANCEMENT FOR PASSIVEly BREATHING PATIENT.

\begin{tabular}{|c|c|c|}
\hline & $\begin{array}{c}\text { IMF Combinations for } \\
\text { CGO }\end{array}$ & $\begin{array}{c}\text { IMF Combinations for } \\
\text { Peso }\end{array}$ \\
\hline EMD & 1 to 3 & 4 to 9 \\
\hline EEMD & 2 to 3 & 4 to 9 \\
\hline
\end{tabular}

From Figure 3.11(A), there is not much difference in terms of the CGO reconstruction morphology between EMD and EEMD during the first 12 seconds. However, like in Section 3.3.3, the main difference is observed during the expiration phase of the low-flow inflation maneuver as indicated by the green box. The red CGO, which was reconstructed using the IMFs from EEMD shows a more consistent CGO morphology compared to the blue CGO, which was reconstructed using EMD's IMFs. The consistent and more defined CGO morphology during the expiration phase seen in EEMD's reconstruction suggests a better separation of the oscillatory modes than the EMD reconstruction. In fact, the frequency smearing seen in the CGO created through EMD's IMFs during expiration is a product of the mode-mixing problem; since the mode-mixing occurred in IMF 1, the subsequent IMFs were modified and did not contain the true information about the physiological process (i.e. the heart beat).

In addition to seeing an improvement in CGO extraction, a noticeable performance improvement of CGO suppression using EEMD in the proposed enhancement scheme was also observed as shown in Figure 3.11(B). At first glance, the EMD reconstruction (blue) appears to suppress the CGO marginally better. While the EEMD reconstruction (red) fluctuates a bit more than its EMD counterpart, the EEMD reconstruction can follow the slope $\left(\frac{d P e s o}{d t}\right)$ of the low-flow inflation significantly better during inspiration and contains less delay during expiration. Ultimately, the EEMD reconstruction's ability to suppress the CGO and reconstruct a more 
accurate representation of the low-flow inflation maneuver outweighs the "better" CGO suppression of EMD.

\subsection{Application of the (Refined) Proposed Enhancement Scheme to Spontaneously Breathing Patients - Another Proof-of-Concept}

Up until now, this Chapter has focused mainly on characterizing the CGO interference and refining the proposed enhancement scheme - both of which were accomplished using data from passively breathing patients. However, there is a push in the critical care community towards developing and adapting modes of ventilation that encourage patients to take on a greater portion of ventilatory effort. As a result, the proposed enhancement scheme must also be robust to conditions where patients are able to recruit their respiratory muscles to provide some degree of ventilation effort (i.e. "active breathing").

The aim of this section is to get a better understanding of the Peso signal in patients who are capable of active breathing, which can be achieved by suppressing their CGO interference. Because an "active breathing" condition is used in this section, which differs from the "passive breathing" condition used in Section 3.3, proof-of-concept results must also be generated to determine the feasibility of the proposed enhancement scheme in conditions other than "passive breathing."

\subsubsection{Dataset}

The dataset used in this section is from spontaneously breathing patients, which are a subset of the "active breathing" condition. Unlike the "passive breathing" condition, the "spontaneously breathing" condition means that the patients' respiratory muscles were not paralyzed; they were able to recruit some of their respiratory muscles to provide some degree of respiratory effort. This 
dataset was obtained from six spontaneously breathing patients receiving pressure support ventilation (PSV). Under the "spontaneously breathing" condition during PSV, the patients' inspiratory efforts triggered the ventilator to provide a pre-set positive pressure to supplement the patients' breath. The dataset contains five signals: i) Airway Pressure $\left[\mathrm{cmH}_{2} \mathrm{O}\right]$, ii) Esophageal Pressure $\left[\mathrm{cmH}_{2} \mathrm{O}\right]$, iii) Gastric Pressure $\left[\mathrm{cmH}_{2} \mathrm{O}\right]$, iv) Flow [L/s], and v) Transpulmonary Pressure $\left[\mathrm{cmH}_{2} \mathrm{O}\right]$. The signals were sampled at $200 \mathrm{~Hz}$, and the duration of the signals range between 5.17 minutes to 8.74 minutes. The collection of this data was approved by St. Michael's Hospital's Research Ethics Board under REB\# 15-369. A summary of the data used in this section is shown in Table 4.

TABLE 4: SUMMARY OF THE DATASET USED FOR REFINING THE PROPOSED ENHANCEMENT SCHEME.

\begin{tabular}{|c|c|c|c|c|c|c|}
\hline $\begin{array}{c}\text { Dataset } \\
\#\end{array}$ & $\begin{array}{c}\text { REB } \\
\#\end{array}$ & $\begin{array}{c}\text { Size of } \\
\text { Dataset } \\
\text { (\# of } \\
\text { patients) }\end{array}$ & $\begin{array}{l}\text { Condition of } \\
\text { Patients }\end{array}$ & Types of data & $\begin{array}{c}\text { Sampling } \\
\text { Frequency } \\
\text { (Hz) }\end{array}$ & $\begin{array}{c}\text { Duration } \\
\text { of Signals } \\
\text { (min) }\end{array}$ \\
\hline 2 & $\begin{array}{c}15- \\
369\end{array}$ & 6 & $\begin{array}{l}\text { Spontaneous } \\
\text { with PSV }\end{array}$ & $\begin{array}{ll}\text { 1. } & \text { Airway Pressure } \\
\text { 2. Esophageal } \\
\text { Pressure } \\
\text { 3. } \\
\text { 4astric Pressure } \\
\text { 5. } & \text { Flow } \\
& \text { Pranspulmonary } \\
& \text { Pressure }\end{array}$ & 200 & $5.17-8.74$ \\
\hline
\end{tabular}

3.5.2 Experimental Setup and Validation - Proof-of-Concept Results for Spontaneously Breathing Patients

As mentioned in the previous section, the dataset being used is from spontaneously breathing patients. Thus, the morphology of the Peso signal will be different from the Peso 
morphology seen in Section 3.3 and Section 3.4, as there will be a negative deflection during inspiration.

The same experimental setup used in Section 3.3 will be applied in this section. Insights on the proof-of-concept results and determining the feasibility of the proposed enhancement scheme will rely on face validity from our clinical collaborators. The specific measurements used for face validity in this section are the IMFs, as well as the quality of the enhanced Peso signal in terms of three specific clinical features: 1) the overall morphology of the Peso, 2) the amplitude of the Peso swing $(\Delta \mathrm{P})$, and 3$)$ the slope of the Peso deflections $\left(\frac{d P e s o}{d t}\right)$.

\subsubsection{Results \& Discussions}

\section{Raw Peso Characteristics}

The Peso signal of a spontaneously breathing patient undergoing PSV in the ICU is shown in Figure 3.12 (left). The Peso swing $(\Delta \mathrm{P})$ ranges between $\sim 6 \mathrm{cmH}_{2} \mathrm{O}$ and $\sim 10.5 \mathrm{cmH}_{2} \mathrm{O}$, and the CGO interference, which appear as small oscillatory pressure fluctuations, occur at quasi-periodic intervals throughout the entire Peso signal. The CGO's morphology is most clearly seen at the plateau occurring at the end of expiration. From visual inspection of the end-expiratory pressure, the CGO appears to have an amplitude of $\sim 1 \mathrm{cmH}_{2} \mathrm{O}$. CGOs are also present during the inspiratory phase (negative deflection of Peso) and expiratory phase (positive deflection of Peso) but are not easy to see, because the significantly larger amplitude of $\Delta \mathrm{P}$ masks the pressure fluctuations of the CGOs.

The frequency-domain representation of the Peso signal is seen in Figure 3.12 (right). The red vertical dotted line separates the activity of the Peso and the CGO; the peaks of the magnitude spectrum to the left of the red line represents the Peso and the DC value, while the peaks to the 
right are representative of the CGO and its harmonics. An interesting observation from the magnitude spectrum is the location of the separating line. Although there are clear indications of the CGO and its harmonics, the position of the separating line lies in an ambiguous region in which there is activity from both the Peso and CGO.
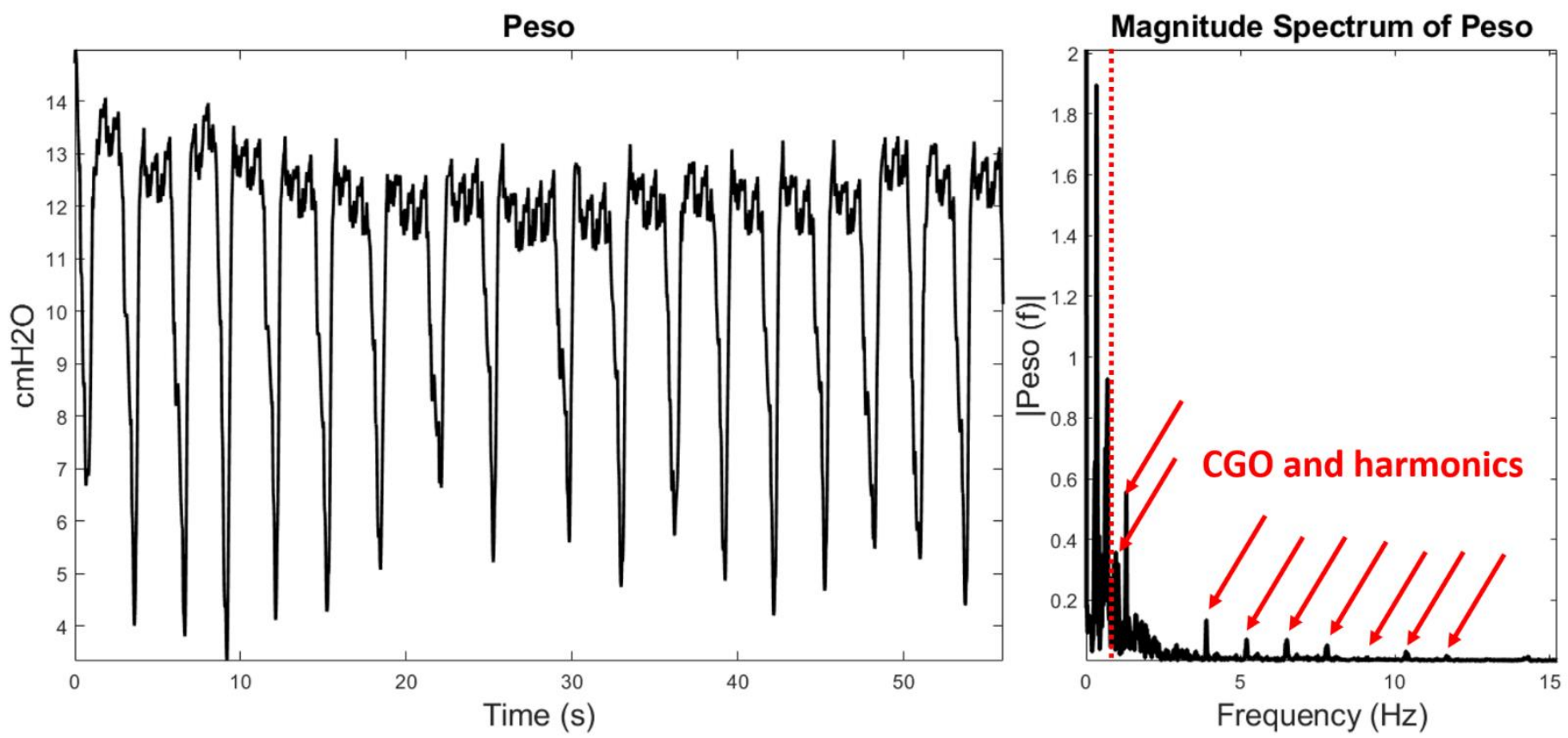

Figure 3.12. The time-domain representation of a Peso from a spontaneously breathing patient (left) and the magnitude spectrum (right). The peaks corresponding to CGO and its harmonics are indicated by red arrows. The red dotted vertical line marks the threshold between Peso activity, which are represented by peaks to the left of the threshold, and the CGO activity. The magnitude spectrum has been zoomed in to get a clear representation of the CGO and its harmonics.

\section{Application of the (Refined) Proposed Enhancement Scheme - A Proof-of-Concept}

\section{Suppressing CGO in Spontaneously Breathing Patients}

To suppress the CGOs and extract the Peso signal, the EEMD algorithm was used to generate the IMFs, which are shown in Figure 3.13. The application of EEMD yielded 11 IMFs with no clear indication of the mode-mixing problem that was observed in Sections 3.3 and 3.4. 


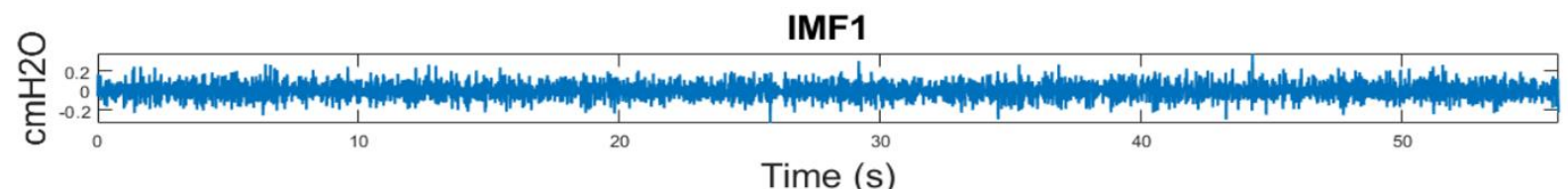

Time (s)

IMF2

Time (s)

IMF3

Time (s)

IN

Time (s)

空 $\mathbb{T}_{-2}^{1}$

Time (s)
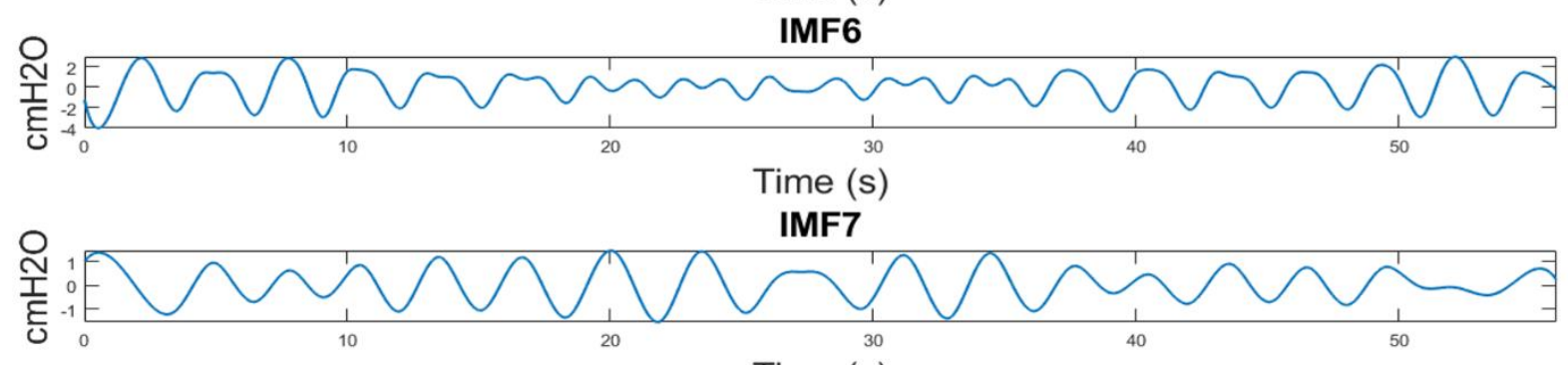

Time (s)

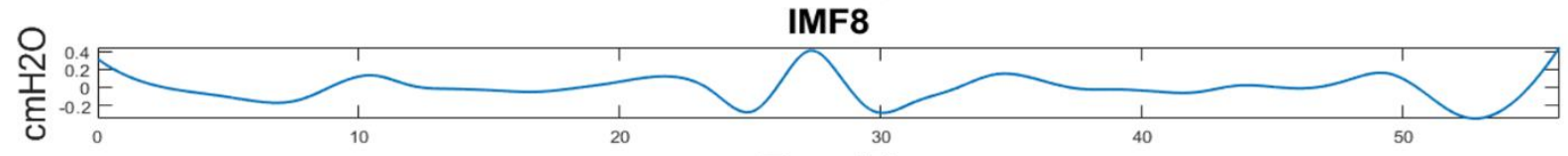

Time (s)

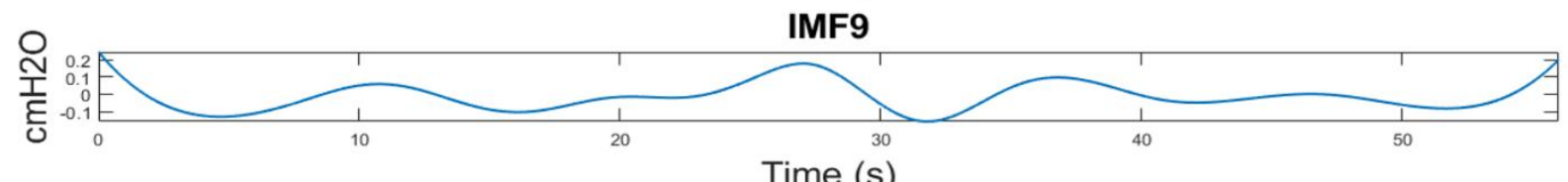

Time (s)

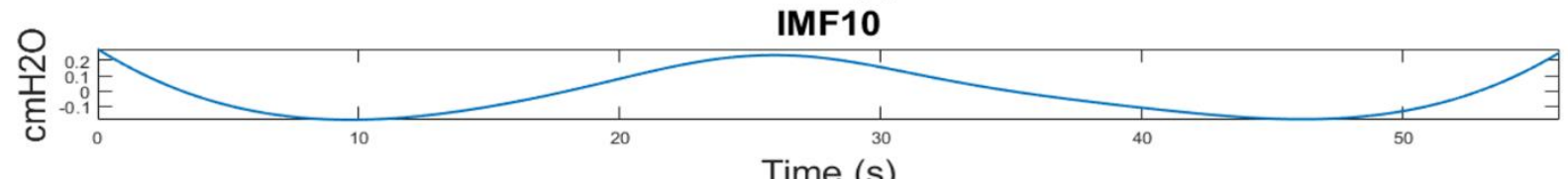

Time (s)

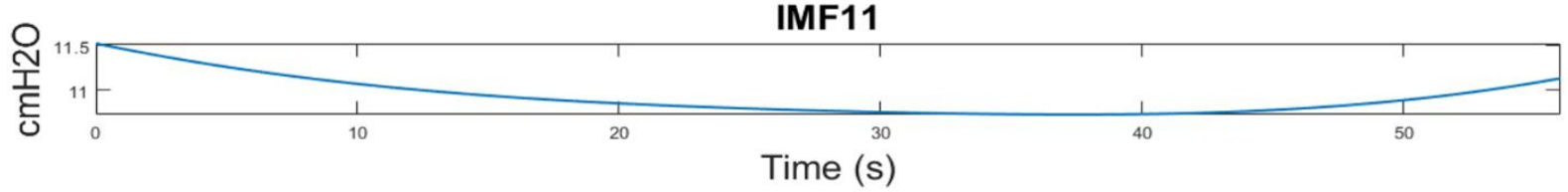

Figure 3.13. The IMFs using EEMD of the Peso for a spontaneously breathing patient. 
Through visual inspection of the IMF morphologies, the clinical collaborators suggested the following IMF combinations for Peso reconstruction:
i) $\quad$ IMF 4 to IMF 11
ii) IMF 5 to IMF 11
iii) IMF 6 to IMF 11

Each of the three reconstructed Peso signals, which were generated using the abovementioned IMF combinations, were plotted separately as seen in Figure 3.14. Figures 3.14 (A), 3.14 (B), and 3.14 (C), correspond to the IMF combinations (i), (ii), and (iii). In order to visually compare the performance of the reconstructions, the reconstructed signals were superimposed onto the original raw Peso and shown to the clinical collaborators.

At first glance, the reconstruction using IMFs 6 to 11 in Figure 3.14 (C) provides the best CGO suppression. However, there are significant modifications to the Peso morphology as well as $\Delta \mathrm{P}$. The raw amplitude of the highest swing within the frame is $\sim 10 \mathrm{cmH}_{2} \mathrm{O}$. Upon reconstruction, $\Delta \mathrm{P}$ was found to be $\sim 6 \mathrm{cmH}_{2} \mathrm{O}$, which is a $4 \mathrm{cmH}_{2} \mathrm{O}$ difference or a $40 \%$ loss of $\Delta \mathrm{P}$ amplitude. Furthermore, there is also a visually significant decrease in $\frac{d P e s o}{d t}$, which manifests as delays in the Peso deflections. It was determined that the loss in amplitude of $\Delta \mathrm{P}$ and the introduction of significant delays in $\frac{d P e s o}{d t}$ resulted in too much clinical information being lost. Thus, Peso reconstruction using IMFs 6 to 11 were deemed unacceptable and clinically non-viable.

The reconstructions of (i) IMFs 4 to 11 and (ii) IMFs 5 to 11 are shown in Figure 3.14(A) and 3.14(B), respectively. The CGO suppression in Figure 3.14(A) shows that the higher frequency components of CGO were removed. However, fluctuations from CGO were still present in the reconstruction. Similar observations were made in Figure 3.14(B) - the high frequency 
components of CGO were removed, but small fluctuations persist albeit less than in 3.14(A). In both cases, there were no significant visual changes in $\frac{d P e s o}{d t}$. Thus, the final clinical feature that was critiqued was the amplitude of $\Delta \mathrm{P}$. The highest $\Delta \mathrm{P}$ in both frames is $\sim 10 \mathrm{cmH}_{2} \mathrm{O}$. The reconstruction using (i) IMFs 4 to 11 shows a negligible difference in $\Delta \mathrm{P}$. On the other hand, the reconstruction using (ii) IMFs 5 to 11 shows a $\Delta \mathrm{P}$ of $\sim 8 \mathrm{cmH}_{2} \mathrm{O}$, which is a $2 \mathrm{cmH}_{2} \mathrm{O}$ difference from the original Peso and an overall $20 \%$ loss of $\Delta \mathrm{P}$ amplitude.

The slight differences in morphology between the reconstruction combinations of (i) and (ii) provided difficulty in determining which reconstruction prevails in the clinical context. To gain more insight into the enhancement performance, the frequency-domain representation of the signals was created. The magnitude spectra of the original Peso and the reconstructed Pesos are shown in Figure 3.15 (A) and 3.15 (B) for reconstructions (i) and (ii), respectively. In both magnitude spectra, most of the peaks associated with CGO and its harmonics have been suppressed. However, as indicated by the green arrow in the spectrum of Figure 3.15 (A), the reconstruction using IMFs 4 to 11 failed to suppress a peak associated with CGO. The same peak in the spectrum of Figure 3.15 (B), which is also indicated by a green arrow, has been significantly reduced in the enhanced Peso comprised of IMFs 5 to 11.

Through face validation of the clinical features consisting of morphology, $\Delta \mathrm{P}$, and $\frac{d P e s o}{d t}$, as well as the magnitude spectrum, it was agreed that the best Peso enhancement was generated using a combination of IMFs 5 to 11 . According to the collaborating clinicians, the proof-ofconcept results portrayed in this section suggest that the proposed enhancement scheme may be a viable solution towards suppressing CGOs in spontaneously breathing patients. 


\section{Limitations of the Proposed Enhancement Scheme}

Although the proof-of-concept showed promising results, two limitations were discovered through preliminary experimentation and application of the proposed enhancement scheme specifically for spontaneously breathing Peso.

The first limitation was that the morphology of the "best" reconstructed Peso, which used the combination of IMFs 5 to 11 , still contained fluctuations from CGO activity. Despite the magnitude spectrum in Figure 3.15 (B) showing that the peaks of CGO and its harmonics have largely been suppressed, the separating line shown in Figure 3.12 was in an ambiguous region between Peso and CGO activity. As a result, there may have been overlapping frequency content between the Peso and CGO that were unable to be separated using EEMD.

The second limitation was the loss in amplitude of $\Delta \mathrm{P}$, which in the case of the "best" Peso reconstruction, was $\sim 2 \mathrm{cmH}_{2} \mathrm{O}$. While there may be a loss of clinical information occurring, the collaborating clinicians suggest that a $\sim 10 \%$ loss in information can be tolerated. The enhanced Peso has a loss of $\sim 20 \%$ in amplitude for $\Delta \mathrm{P}$. However, it has also been suggested by the clinicians that the CGO may be modifying the amplitude of $\Delta \mathrm{P}$ during the negative and positive deflections observed during inspiration and expiration. Thus, there may be a possibility that the $20 \%$ loss in $\Delta \mathrm{P}$ has been overestimated, because the "baseline" or "ground-truth" signal, $\mathrm{P}_{\text {meas }}$, is a noisy signal consisting of the CGO interference. 

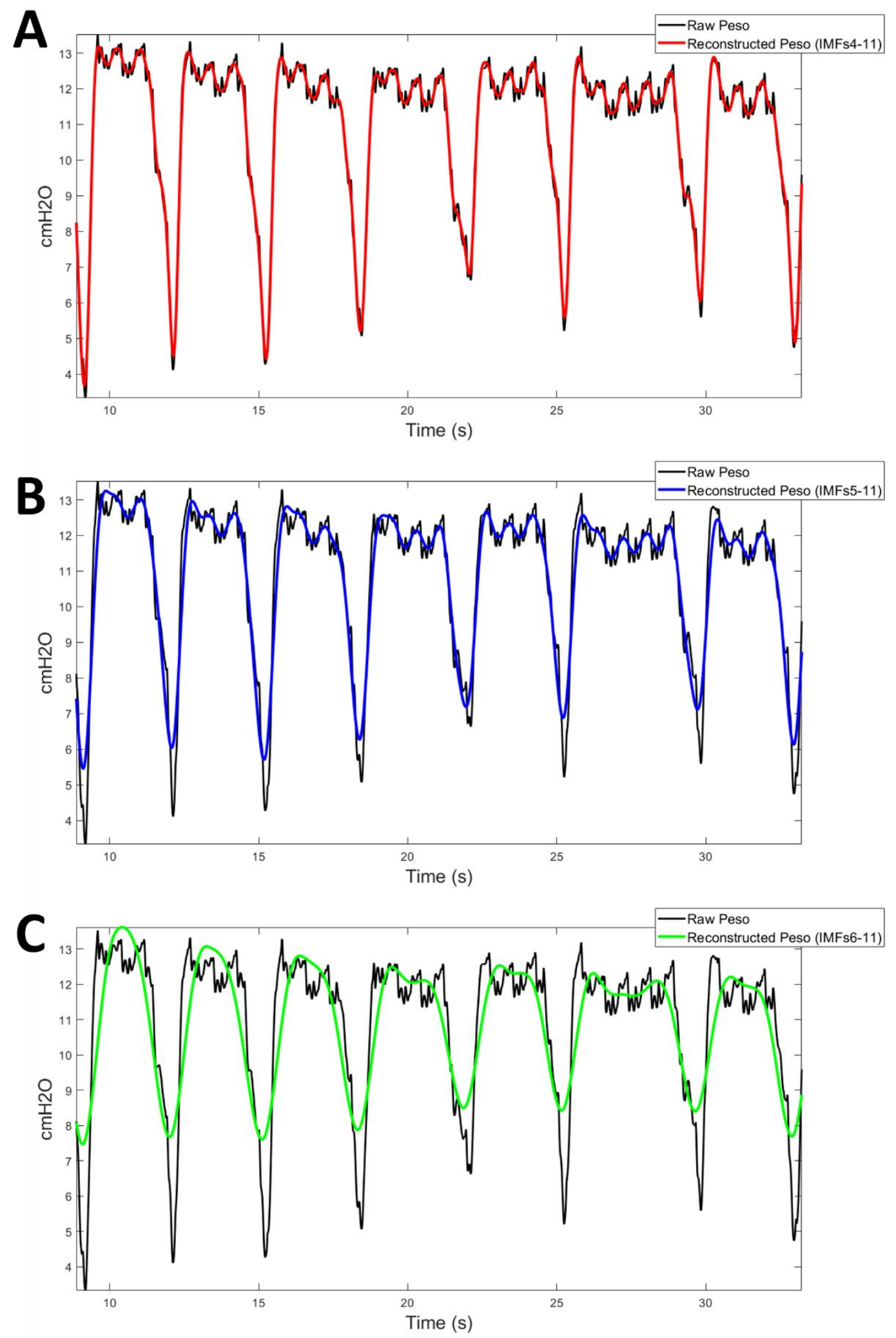

Figure 3.14. A comparison of the raw Peso (black) and the reconstructed Peso using the following IMF combinations: (A) IMFs 4 to 11 (red) (B) IMFs 5 to 11 (blue) (C) IMFs 6 to 11 (green). 

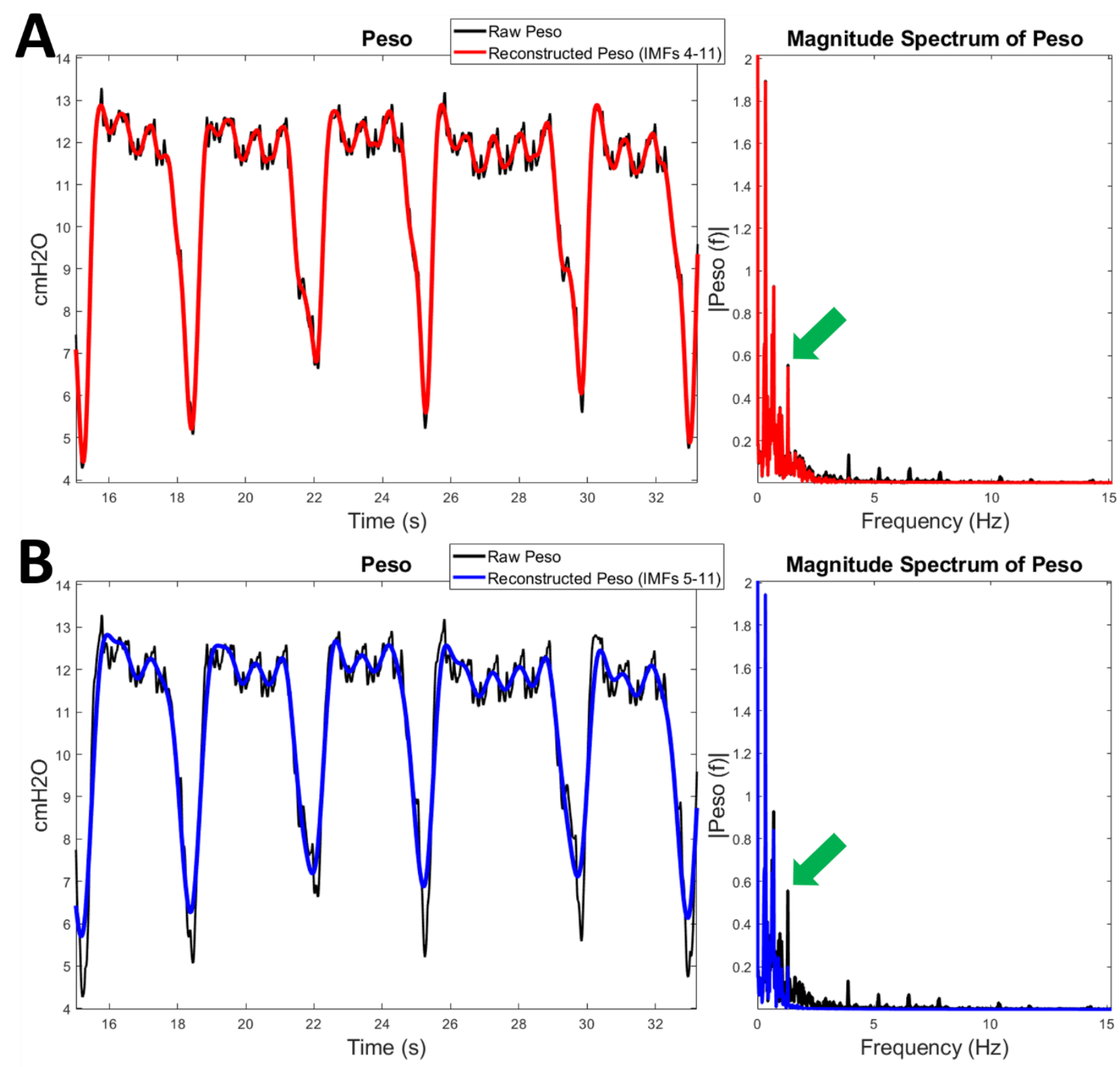

Figure 3.15. (A) The time-domain representations of the Peso signals (left) and the corresponding magnitude spectra (right). The raw Peso is shown in black while the reconstructed Peso using IMFs 4 to 11 are plotted in red. The peak indicated by the green arrow shows that the activity from the CGO has not been suppressed. (B) Similarly, the time-domain (left) and frequency-domain (right) representations of the Peso signals are shown. The raw Peso is plotted in black and the reconstructed Peso using IMFs 5 to 11 are shown in blue. The green arrow in $(\mathbf{B})$ points to the CGO peak described previously in (A). The reconstructed Peso (blue) has significantly reduced the magnitude of the peak. 


\subsection{Chapter Summary}

This chapter introduced the proposed enhancement scheme that would be used to suppress CGO interference in the Peso signal of a patient in the ICU. However, the proposed enhancement scheme requires that the signal first be decomposed into its constituent components (i.e. IMFs) using EMD or EEMD. Thus, characterizing the CGO, the Peso, and the IMFs was the necessary first step in determining the feasibility of the proposed enhancement scheme.

In Section 3.3, data from passively breathing patients in the ICU was used to characterize the CGO interference in the time- and frequency-domains. Paralysis of the patients' respiratory muscles were induced to suppress their respiratory drive. Thus, only the CGO activity and a lowflow inflation maneuver delivered at a constant flow rate by the mechanical ventilator were present in the Peso signal. The EMD algorithm was then used to generate the IMFs of the measured Peso signal. Several combinations of IMFs selected and tested to accomplish two tasks: i) the reconstruction of CGO, and ii) the reconstruction of Peso. The proof-of-concept results using the proposed enhancement scheme shown in in this section suggest that CGO extraction as well as CGO suppression can be achieved. Despite the results, further inspection of the IMFs found that the mode-mixing problem was present when EMD was chosen as the decomposition algorithm. The mode-mixing problem was alluded to in Section 3.2.1, which suggests that the resulting IMFs lose their physical meaning. The loss in physical meaning is because EMD is sequential in nature; the subsequent IMFs depend on the previous IMF. Thus, mode-mixing poses a problem in applications in source-separation applications such as the one presented in this thesis.

To overcome the mode-mixing problem, Section 3.4 was dedicated to comparing the IMFs between the EMD and EEMD algorithms. The EEMD algorithm was applied to the same data used in Section 3.3, and it was found that the mode-mixing was no longer present. The CGO and Peso 
signals were then reconstructed using EEMD's IMFs and compared to the reconstructions using EMD. Ultimately, the reconstructions of the passively breathing Peso using IMFs from EEMD were significantly better in retaining valuable clinical information. Thus, EEMD was selected as the decomposition algorithm used for the proposed enhancement scheme. Furthermore, feedback from the clinical collaborators found that the proposed enhancement scheme may be useable for Peso signals from passively breathing patients.

The refined enhancement scheme using EEMD was then applied to spontaneously breathing patients in Section 3.5. The application of the proposed enhancement scheme to spontaneously breathing patients is important, because many clinicians are now trying to develop modes of ventilation that encourage patients to take on a greater proportion of the ventilatory effort. Thus, the technique must be robust to breathing conditions in which patients' respiratory drives are active. In this section, the morphology of the Peso signals used were found to be more complex, which meant that process of selecting the "best" Peso reconstruction was more reliant on the clinicians' opinions. However, the subjective input from multiple clinicians brought with it an interesting challenge. While some clinicians may take a more conservative approach to CGO suppression, there are others who may be more comfortable with a more liberal amount of filtering. Nevertheless, the group of collaborating clinicians were able to converge on a "best" reconstruction, which was presented in this section.

In this chapter, the CGO and Peso signals were characterized in both time-domain and frequency-domain for passively breathing patients as well as spontaneously breathing patients. For both breathing conditions, the CGO were able to be suppressed as agreed upon by the collaborating clinicians. Interestingly, preliminary experiments into characterizing the CGO also suggest that the proposed enhancement scheme is also capable of extracting the CGO signal. From the findings 
obtained in this chapter, the final enhancement scheme can be represented by the block diagram shown in Figure 3.16.

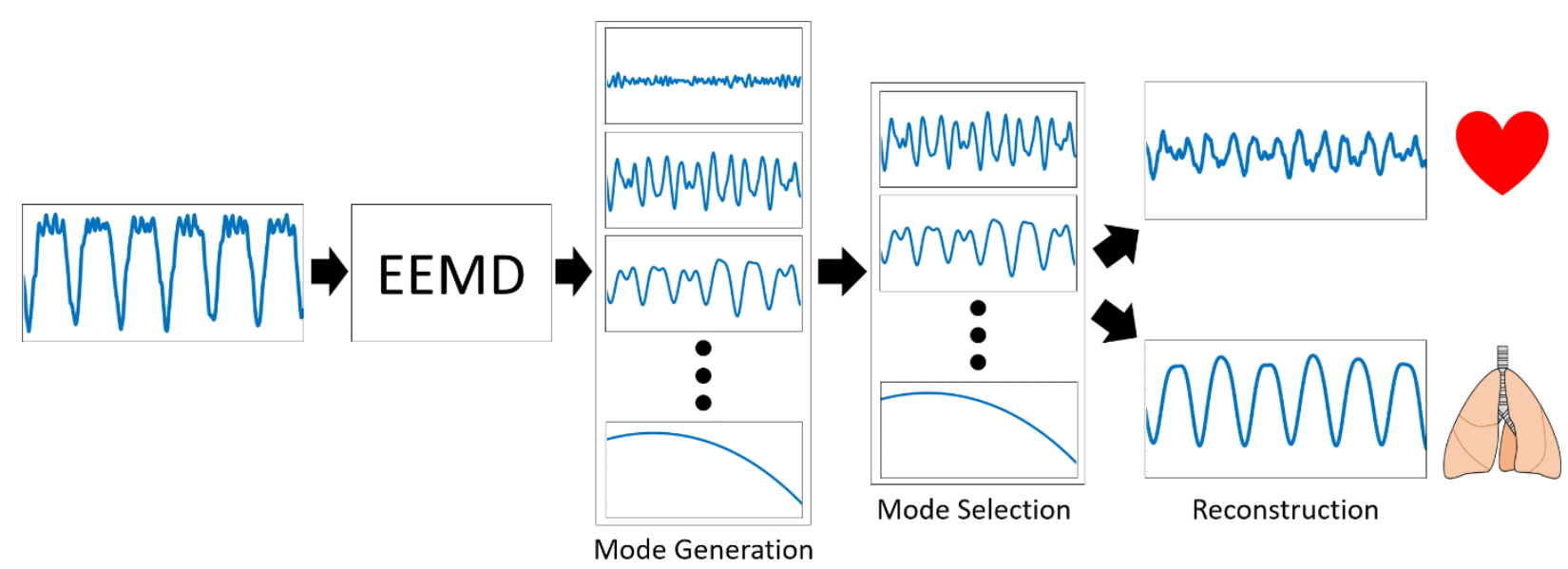

Figure 3.16. The block diagram of the refined enhancement scheme used to suppress CGO. The EEMD algorithm is chosen to decompose the raw Peso signal into its IMFs. Through mode selection, it is possible to reconstruct both the CGO and the Peso separately.

This chapter has also brought to light some major challenges in developing signal enhancement techniques for biomedical signals such as Peso. The "gold-standard" for validation is through subjective visual assessment (i.e. face validation) by domain-experts such as clinicians. The subjective assessments rely on clinical opinions, which means that the performance of the enhancement techniques can depend on whether individual clinicians are able to tolerate more filtering or are more comfortable with less filtering. Another challenge is that it is difficult to objectively benchmark the performance of any enhancement technique, because the baseline or “clean" Peso signals from live ICU patients are impossible to collect. Additionally, the subjective assessments of domain-experts may contain bias since the true morphology of the Peso signal (e.g. the positive and negative deflections) has already been modified by the CGO interference. Finally, the number of Peso signals available to develop and test the proposed enhancement scheme is limited, resulting in a "small dataset" problem. 


\section{Chapter 4}

\section{FrankEinstein's Peso Signals}

\subsection{Motivation}

At the end of the last Chapter, three key challenges were summarized and have to be taken into account during the development and validation of biomedical signal enhancement techniques specifically for Peso signals:

1. The gold-standard for validation is subjective and based on visual assessment/face validation by clinical experts,

2. There are no objective or quantifiable approaches to benchmark the performance of the developed technique, and

3. The fidelity of face validation for clinical parameters such as $\Delta \mathrm{P}$ can be compromised since the Peso signal has already been modified by the CGO.

In addition to the abovementioned challenges, the previous Chapter also brought up a "small database" problem that imposed limitations on developing and testing robust signal enhancement techniques. The aim of this Chapter is to address these challenges and limitations by applying the proposed enhancement scheme to synthetic "noisy" Peso signals.

Inspired by Mary Shelley's science-fiction novel Frankenstein; or, The Modern Prometheus, which tells the story of a young scientist who pieces together different body parts and reanimates a living "human," synthetic "noisy" Peso signals were created. In a similar vein to the creation of Frankenstein's Monster, the synthetic "noisy" Peso signals were created by piecing 
together two elements: i) Peso signals from a breathing simulator, and ii) CGO signals that were extracted using EEMD from patients as described in the previous Chapter. The synthetic "noisy" Peso signals were "reanimated" using EQ4.1:

$$
P_{\text {eso }}=P_{\text {eso }, \text { simulated }}+C G O_{\text {extracted }} \quad(\text { EQ4. 1) }
$$

These synthetic Peso signals are endearingly termed, "FrankEinstein’s Peso signals." This Chapter will go into detail about the elements and methodology used to generate FrankEinstein's Peso signals. The proposed enhancement scheme will be then be applied to FrankEinstein's Peso signals, and the performance of the enhancement will be quantified through error measurements.

\subsection{Simulating Peso Signals}

Benchmarking and objectively quantifying the performance of any biomedical signal enhancement technique ideally requires a "clean" version of the signal being enhanced. However, "clean" signals are oftentimes impossible to collect due to the inherent coupling of the human body's physiological systems. In the context of this thesis, the relative anatomical positioning and physiological interactions between the respiratory and cardiovascular systems pose a challenge in monitoring solely the mechanics of the respiratory system. While it may be impossible to obtain a CGO-free Peso signal from a spontaneously breathing person, there are lung model simulators in the market that are able to realistically simulate human respiratory mechanics and provide respiratory signals that are seen in the clinic. The respiratory signals obtained from the simulator, which includes Peso signals, can then be used as reference or "clean" signals.

\subsubsection{Overview of Simulator}

The breathing simulator used in this work is the IngMar Medical ASL 5000 (Active Servo Lung) shown in Figure 4.1. The ASL 5000 is capable of not only simulating and reproducing 
signals from passively breathing patients but is also able to simulate the respiratory mechanics of spontaneously breathing patients.

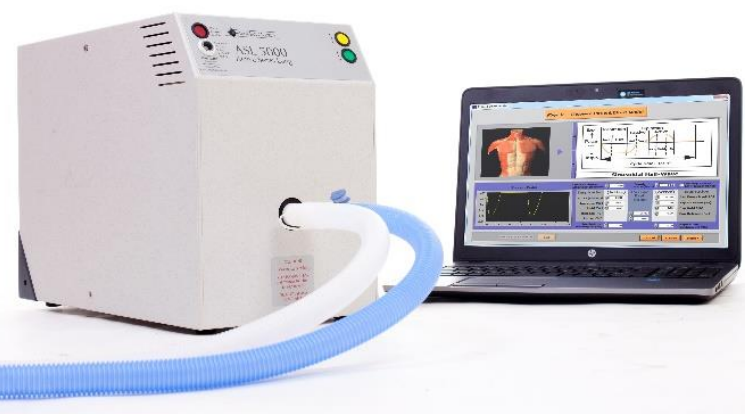

Figure 4.1. The IngMar Medical ASL 5000 (Active Servo Lung). Source: [59]

The simulation data obtained from the ASL 5000 is based on the movement of a piston within a cylinder that generates pressure. The piston's movements depend on the respiratory model parameters that are defined on the computer as well as the equations of motion of respiration for either passively breathing or spontaneously breathing conditions (more details about the equations of motion can be found in [18], [31], and [59]). Figure 4.2 illustrates the functional overview of the ASL 5000 [59].

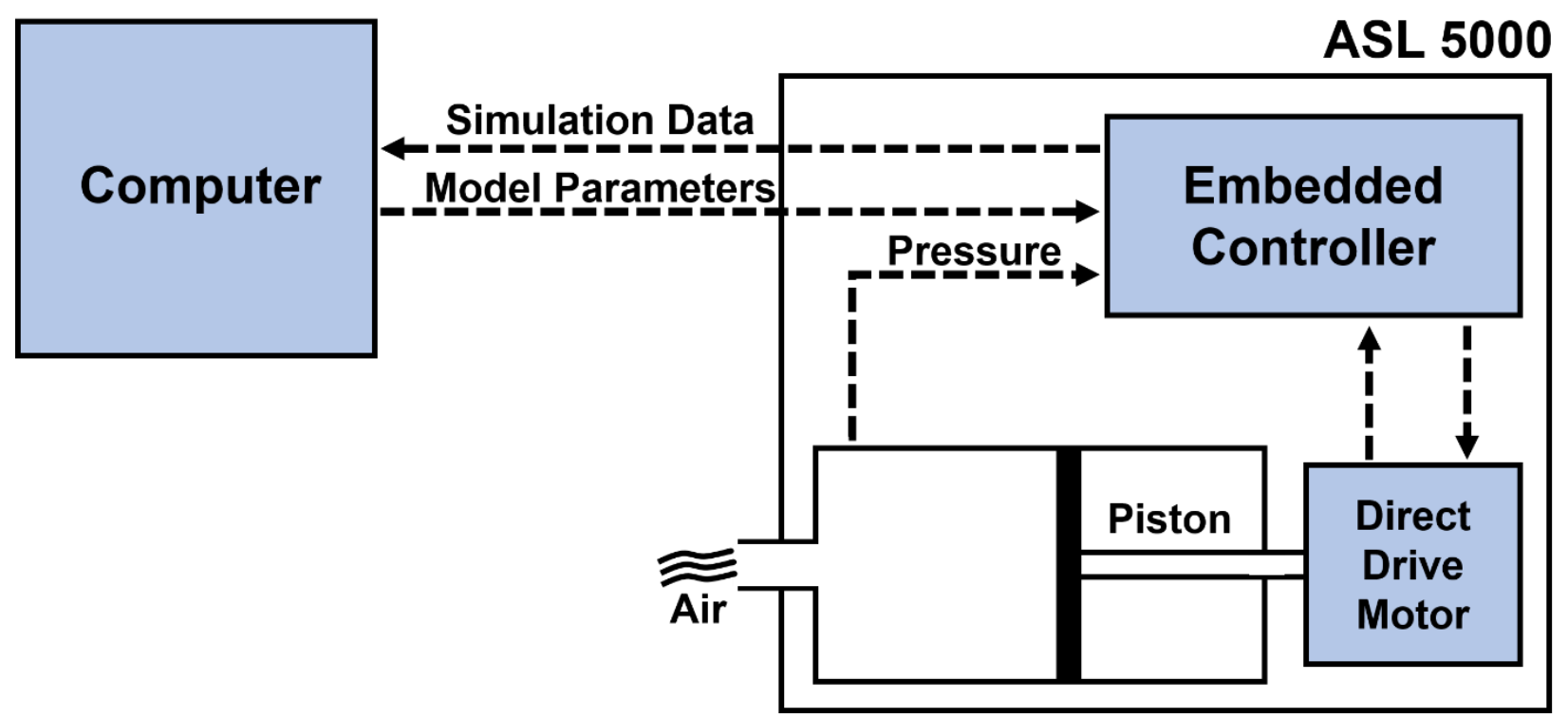

Figure 4.2. A functional overview of the ASL 5000. Source: Adapted from [59]. 


\subsubsection{Simulating the Peso Signal}

As mentioned in the previous Chapter, there is a push in the critical care community to develop modes of ventilation that encourage patients to take on a greater proportion of the ventilatory effort. Thus, rather than simulating passively breathing patients, it was decided that the FrankEinstein Peso signals would be simulated as spontaneously breathing patients. However, to simulate Peso signals that mimic spontaneously breathing patients in the ICU, two models had to be defined in the ASL 5000 software: i) the lung model, and ii) the patient-effort model.

\section{Lung Model}

For the purposes of generating FrankEinstein's Peso signals, the single compartment model for the lungs was selected for simplicity. The single compartment model of the lung and its equivalent analog circuit are shown in Figure 4.3. From the analog circuit equivalent, it can be seen that the single compartment model consists of one resistor, which can have linear or parabolic properties, and one linear capacitor (i.e. compliance). The governing first-order differential equation shown in EQ4.2, which is based on the RC-circuit shown in Figure 4.3, can be used to represent $\Delta \mathrm{P}_{\text {tot }}$, which is the total effective pressure driving the respiratory system [59]. The convention used to formulate EQ4.2 is such that inflation of the lungs is positive and deflation of the lungs is negative.

$$
\Delta P_{t o t}=\left(P_{A O}-P_{B S}\right)+\Delta P_{m u s}=\frac{V}{C}+R \dot{V}
$$


The parameters in EQ4.2 are defined as follows:

$\mathrm{P}_{\mathrm{AO}}$ is the pressure at the airway opening $\left[\mathrm{cmH}_{2} \mathrm{O}\right]$

$\mathrm{P}_{\mathrm{BS}}$ is the pressure at the surface of the body $\left[\mathrm{cmH}_{2} \mathrm{O}\right]$

$\Delta \mathrm{P}_{\text {mus }}$ is the internal pressure caused by the patient's effort [ $\left.\mathrm{cmH}_{2} \mathrm{O}\right]$

$\mathrm{V}$ is the volume of the lungs [L]

$\dot{\mathrm{V}}$ is the flow $[\mathrm{L} / \mathrm{s}]$

$\mathrm{C}$ is the compliance of the lungs $\left[\mathrm{mL} / \mathrm{cmH}_{2} \mathrm{O}\right]$

$\mathrm{R}$ is the resistance of the airway $\left[\mathrm{cmH}_{2} \mathrm{O} /(\mathrm{L} / \mathrm{s})\right]$

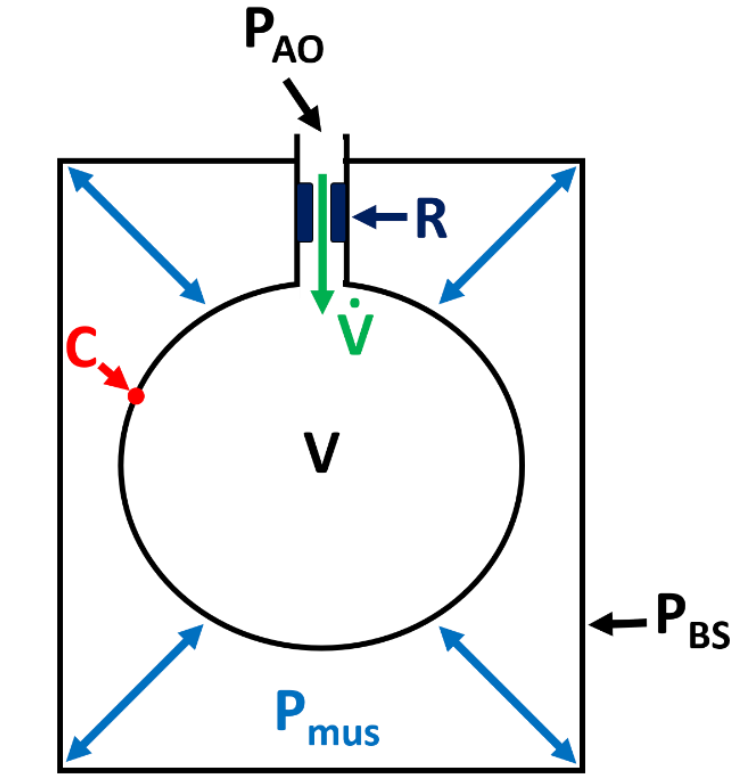

Single Compartment Model

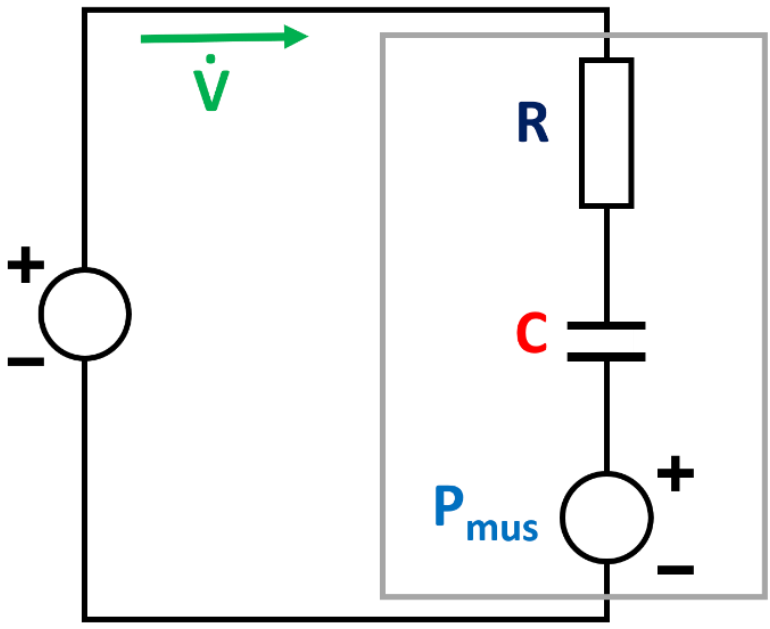

Analog Circuit
Equivalent

Figure 4.3. The single compartment model of the lungs used in generating FrankEinstein's Peso signals (left) and the equivalent analog circuit equivalent (right). The single compartment model consists of a one linear or parabolic resistor (R), and one linear compliance (C). Source: Adapted from [59]. 
The single compartment model is dependent on three mechanical properties: i) the resistance of the airway $(\mathrm{R})$ and ii) the compliance of the lungs $(\mathrm{C})$, both of which can be modified by the user, and iii) the time-constant $(\tau)$, which is the product of R and C. According to [59], the single compartment system modelled by EQ4.2 assumes that both the right and left lungs act together as a single, pneumatic, visco-elastic compartment, which means that its mechanical properties are uniformly distributed across both lungs. Furthermore, EQ4.2 is a system with one degree-of-freedom, which is characterized by a single time-constant. In the clinical context, the single time-constant is representative of both lungs inflating and deflating in unison.

The final component of the single compartment model is $\mathrm{P}_{\text {mus, }}$ which is shown as an independent source in the analog circuit equivalent. $\mathrm{P}_{\text {mus }}$ represents the internal pressure generated by the patient's respiratory effort. It is important to note that the traces of $\mathrm{P}_{\text {mus }}$ are plotted as the negative of $\mathrm{P}_{\text {mus }}$ (i.e. $-\mathrm{P}_{\mathrm{mus}}$ ), which resembles the Peso signal [59]. Thus, $\mathrm{P}_{\text {mus }}$ is used as FrankEinstein's Peso signal component. The distinction of $\mathrm{P}_{\text {mus }}$ being plotted as $-\mathrm{P}_{\text {mus }}$ is based on the convention used to formulate EQ4.2 in that a positive value indicates lung inflation (i.e. inspiration). However, recall that the Peso signal (represented by $\mathrm{P}_{\text {mus }}$ here) has a negative deflection during inspiration for spontaneously breathing patients according to Boyle's Law as described back in Chapter 3. Because the Peso is an independent source and represented by the patient's effort, it must be defined under the patient-effort model as a separate parameter to the ASL 5000.

\section{Patient-Effort Model}

As mentioned previously, the Peso signals are defined in the patient-effort model parameters. The morphology and patterns of the signals are pre-defined in the ASL 5000 software. 
Because the aim of FrankEinstein's Peso signals is to simulate Peso signals that are reminiscent of the ones seen in spontaneously breathing ICU patients, the sinusoidal breath profile was selected.

The sinusoidal breath profile is defined primarily by five parameters:

1. Peak (negative) Pressure: the amplitude of the Peso swing $(\Delta \mathrm{P})$ (inspiration phase)

2. Breath Frequency (breaths/min): the simulated breathing rate

3. Time \% of Pressure Drop: the percentage of one full breath cycle (100\%) required to reach the peak negative pressure (inspiration phase)

4. Time \% of Hold: the percentage of one full breath cycle (100\%) allocated to maintaining the peak negative pressure (inspiration phase)

5. Time \% of Release: the percentage of one full breath cycle (100\%) required to return from the peak negative pressure back to zero (passive expiration)

In addition to the five parameters, the sinusoidal breath model can also be used to simulate active expiration, which means that the person is recruiting their respiratory muscles to force more air out of their lungs. While active or forced expiration tests are often used to diagnose asthma or chronic obstructive pulmonary disease (COPD), in the context of this work, forced expiration can mimic poor patient-ventilation interaction in which the patient fights the ventilator [59]. The active expiration parameters include the: 1) peak (positive) pressure, 2) time $\%$ of increase, 3 ) time $\%$ of hold, and 4) time $\%$ of release. The eight parameters describing the sinusoidal breath model are visually illustrated in Figure 4.4. 


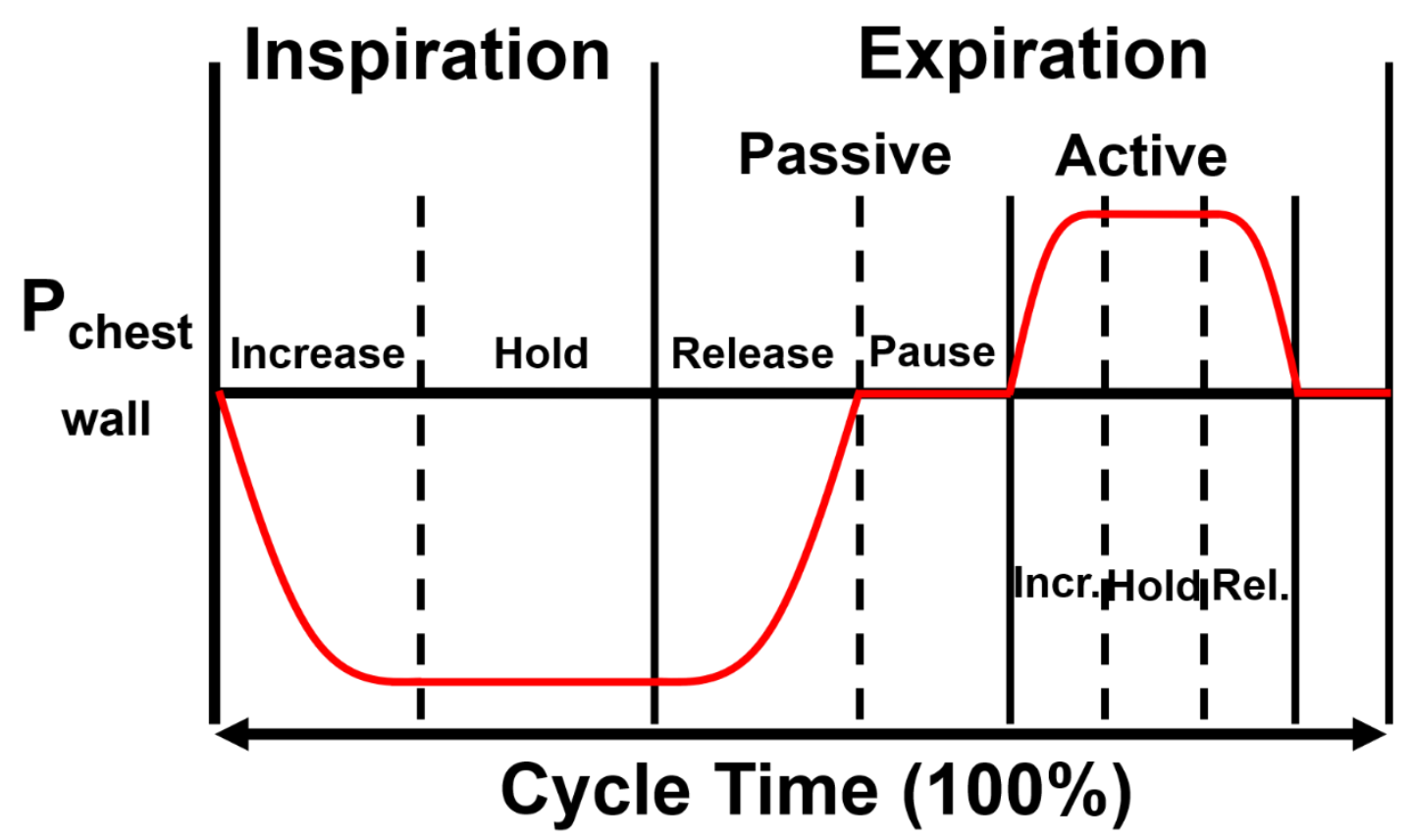

Figure 4.4. An illustration of eight of the nine parameters used to define the $\mathrm{P}_{\text {mus }} /$ Peso trace (red) for the sinusoidal breath model for one full breath cycle. Not pictured in this figure is the breathing rate. Source: Adapted from [59].

In the patient-effort model specifically used to create FrankEinstein's Peso signals, the Peso signal comprised of only an inspiration phase and a passive expiration phase. The inspiration phase was split into $20 \%$ increase time and a $3 \%$ hold of the peak pressure, which was set to -10 $\mathrm{cmH}_{2} \mathrm{O}$. Thus, the inspiration phase accounted for $23 \%$ of the entire breath cycle. On the other hand, the expiration phase made up $77 \%$ of the breath cycle with $15 \%$ release time, which represents the return from peak pressure of inspiration to zero, and $62 \%$ hold for the remaining breath cycle. An illustration of FrankEinstein's breath profile is shown in Figure 4.5 and the parameters of the patient-effort model are summarized in Table 5. 
TABLE 5: PATIENT-EFFORT MODEL PARAMETERS USING SINUSOIDAL BREATH PROFILE FOR FRANKEINSTEIN'S PESO.

\begin{tabular}{|c|c|}
\hline \multicolumn{3}{|c|}{ Inspiration } \\
\hline Peak Pressure & $-10 \mathrm{cmH}_{2} \mathrm{O}$ \\
\hline Increase & $20 \%$ \\
\hline Hold & $3 \%$ \\
\hline \multicolumn{2}{|c|}{ Passive Expiration } \\
\hline Release & \\
\hline Hold & $15 \%$ \\
\hline
\end{tabular}

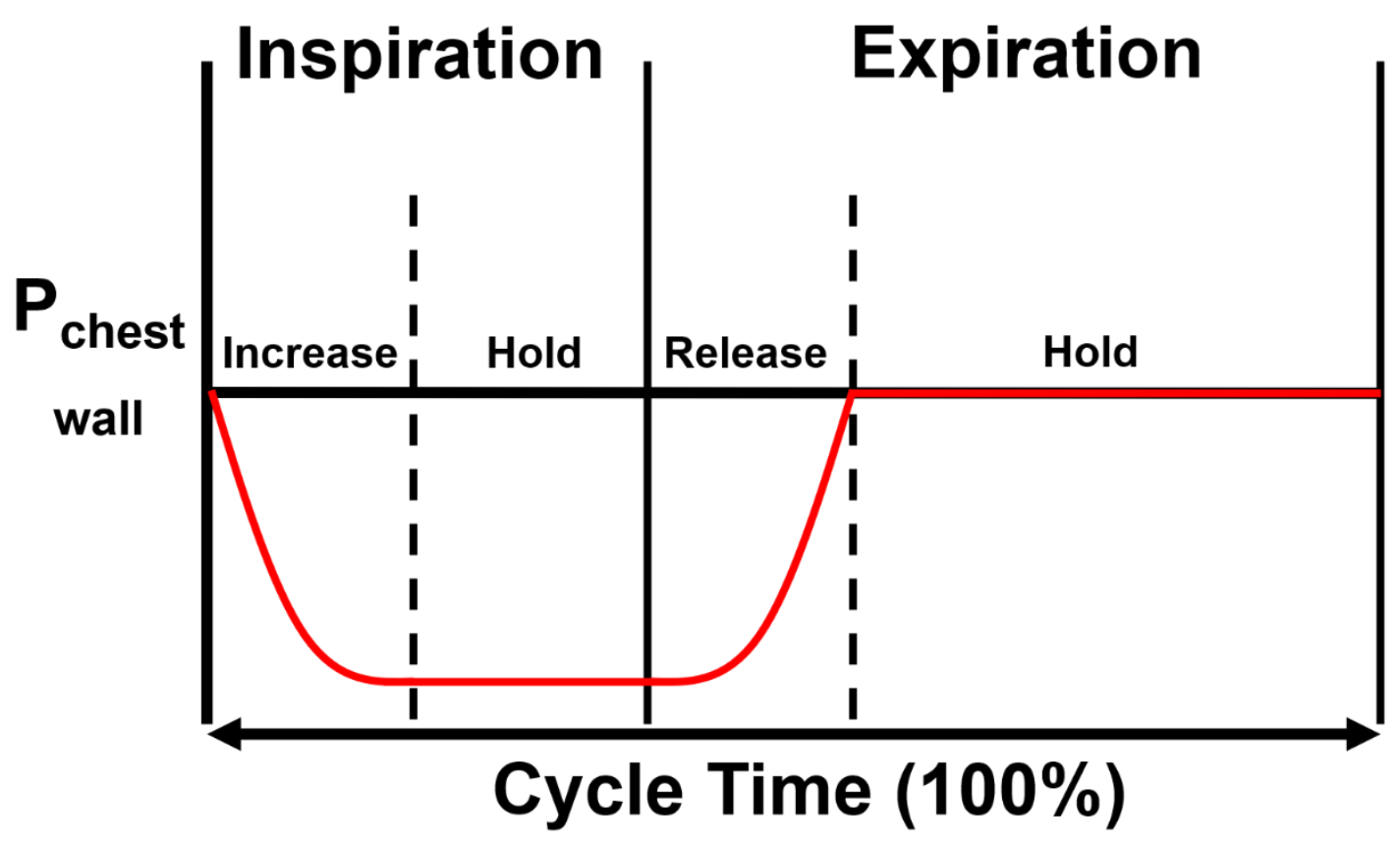

Figure 4.5. An illustration of the FrankEinstein's Peso trace (red) using the sinusoidal breath model for one full breath cycle. Note that the illustration is not to scale.

\subsubsection{FrankEinstein's “clean” Peso Signals}

\section{Acquisition of Peso from ASL 5000}

With the lung and patient-effort models defined, the final step in generating the "clean" Peso was to run the models through the ASL 5000 and collect the simulated data. All data was sampled at $256 \mathrm{~Hz}$. Eight Peso signals were simulated at different respiratory rates while 
maintaining the values of peak pressure of $\Delta \mathrm{P}$ at $-10 \mathrm{cmH}_{2} \mathrm{O}$, the functional residual capacity (FRC) at $500 \mathrm{~mL}$, the compliance of the lung at $20 \mathrm{~mL} / \mathrm{cmH}_{2} \mathrm{O}$, and the resistance of the airways at $10 \mathrm{cmH}_{2} \mathrm{O} / \mathrm{mL}$. One peso signal was simulated at a respiratory rate of 15 breaths/min and varying the peak pressure of $\Delta \mathrm{P}$ between -5 to $-15 \mathrm{cmH}_{2} \mathrm{O}$. The other parameters were kept constant. The duration of each simulation was set to five minutes, but there were trials resulting in the ASL 5000 crashing after four minutes. Nevertheless, the data was kept. In total, nine "clean" Peso signals were created for FrankEinstein's Peso signals. A summary of the nine Peso signals and their associated parameters are shown in Table 6.

Table 6: Parameters used to simulate “CLEAN” Peso On the ASL 5000.

\begin{tabular}{|c|c|c|c|c|c|c|}
\hline $\begin{array}{c}\text { Peso } \\
\text { Signal \# }\end{array}$ & $\begin{array}{c}\text { Respiratory } \\
\text { Rate } \\
\text { (breaths/min) }\end{array}$ & $\begin{array}{l}\operatorname{Max} \Delta P \\
\left(\operatorname{cmH}_{2} \mathrm{O}\right)\end{array}$ & $\begin{array}{l}\text { FRC } \\
(\mathrm{mL})\end{array}$ & $\begin{array}{c}\text { Compliance } \\
\left(\mathbf{m L} / \mathbf{c m H}_{2} \mathrm{O}\right)\end{array}$ & $\begin{array}{c}\text { Resistance } \\
\left(\mathrm{cmH}_{2} \mathrm{O} / \mathrm{mL}\right)\end{array}$ & $\begin{array}{c}\text { Duration } \\
\text { (mins) }\end{array}$ \\
\hline 1 & 15 & -10 & 500 & 20 & 10 & 5 \\
\hline 2 & 17 & -10 & 500 & 20 & 10 & 5 \\
\hline 3 & 19 & -10 & 500 & 20 & 10 & 5 \\
\hline 4 & 21 & -10 & 500 & 20 & 10 & 5 \\
\hline 5 & 23 & -10 & 500 & 20 & 10 & 4 \\
\hline 6 & 27 & -10 & 500 & 20 & 10 & 5 \\
\hline 7 & 29 & -10 & 500 & 20 & 10 & 5 \\
\hline 8 & 31 & -10 & 500 & 20 & 10 & 5 \\
\hline 9 & 15 & -5 to -15 & 500 & 20 & 10 & 4 \\
\hline $\begin{array}{l}\text { Sampling } \\
\text { Frequency } \\
(\mathbf{H z})\end{array}$ & \multicolumn{6}{|c|}{256} \\
\hline
\end{tabular}

Two Peso signals are shown in Figure 4.6 and 4.7 to illustrate the Peso signals obtained from the ASL 5000. Figure 4.6 is a Peso signal corresponding to Peso Signal \#1 from Table 6 with a respiratory rate of 15 breaths $/$ min and a $\max \Delta \mathrm{P}$ of $-10 \mathrm{cmH}_{2} \mathrm{O}$ while keeping the other parameters constant. Figure 4.7 is representative of Peso Signal \#9 from Table 6 with a respiratory rate of 15 breaths/min and a max $\Delta \mathrm{P}$ being varied between -5 to -15 . The other parameters were also kept constant. 


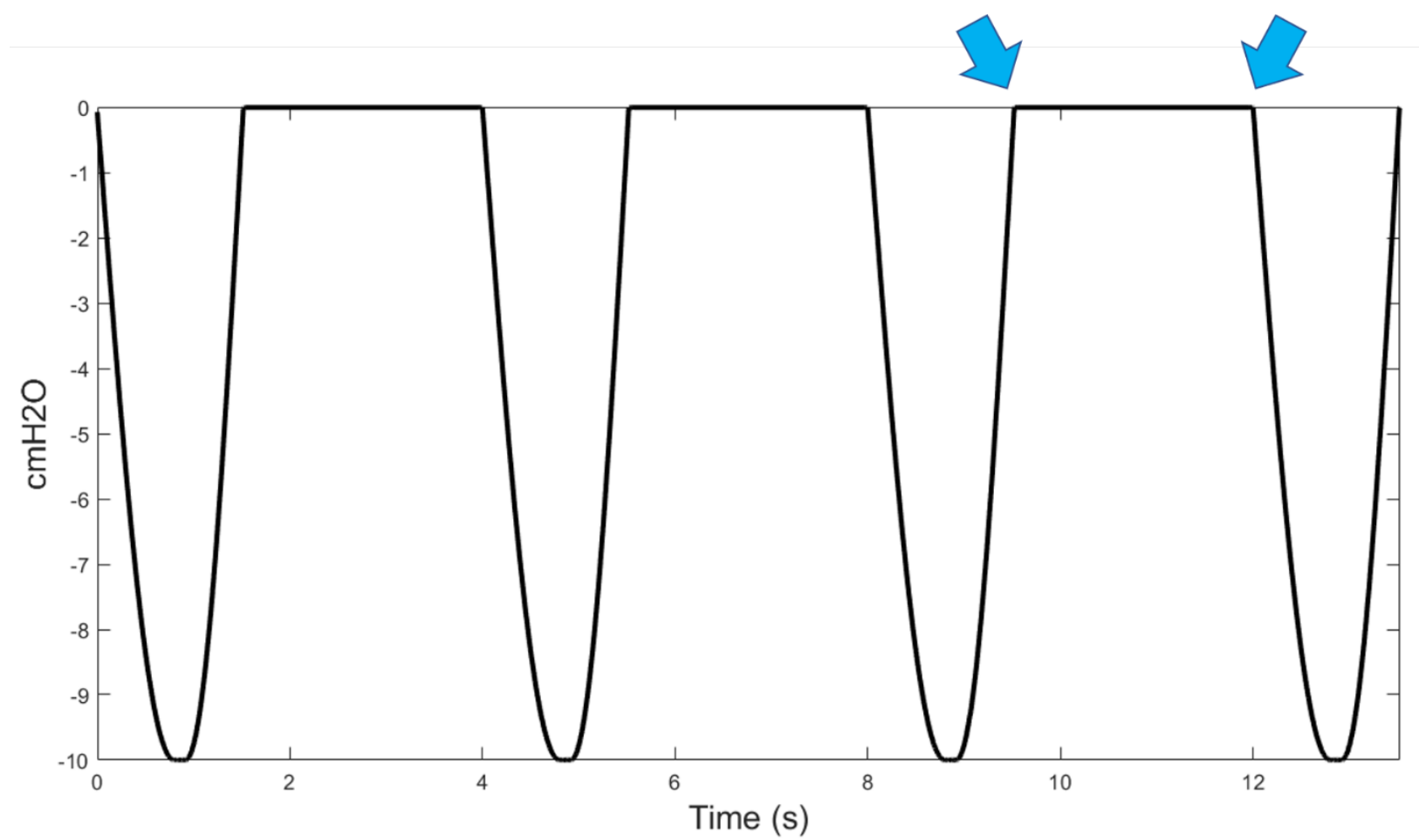

Figure 4.6. The "clean" Peso signal simulated from the ASL 5000. The max pressure amplitude of $\Delta \mathrm{P}$ is $-10 \mathrm{cmH}_{2} \mathrm{O}$. The blue arrows indicate a sharp inflection at the transition between i) the end of expiratory hold and the beginning of inspiratory increase, and ii) the end of expiration release and the beginning of expiration hold.

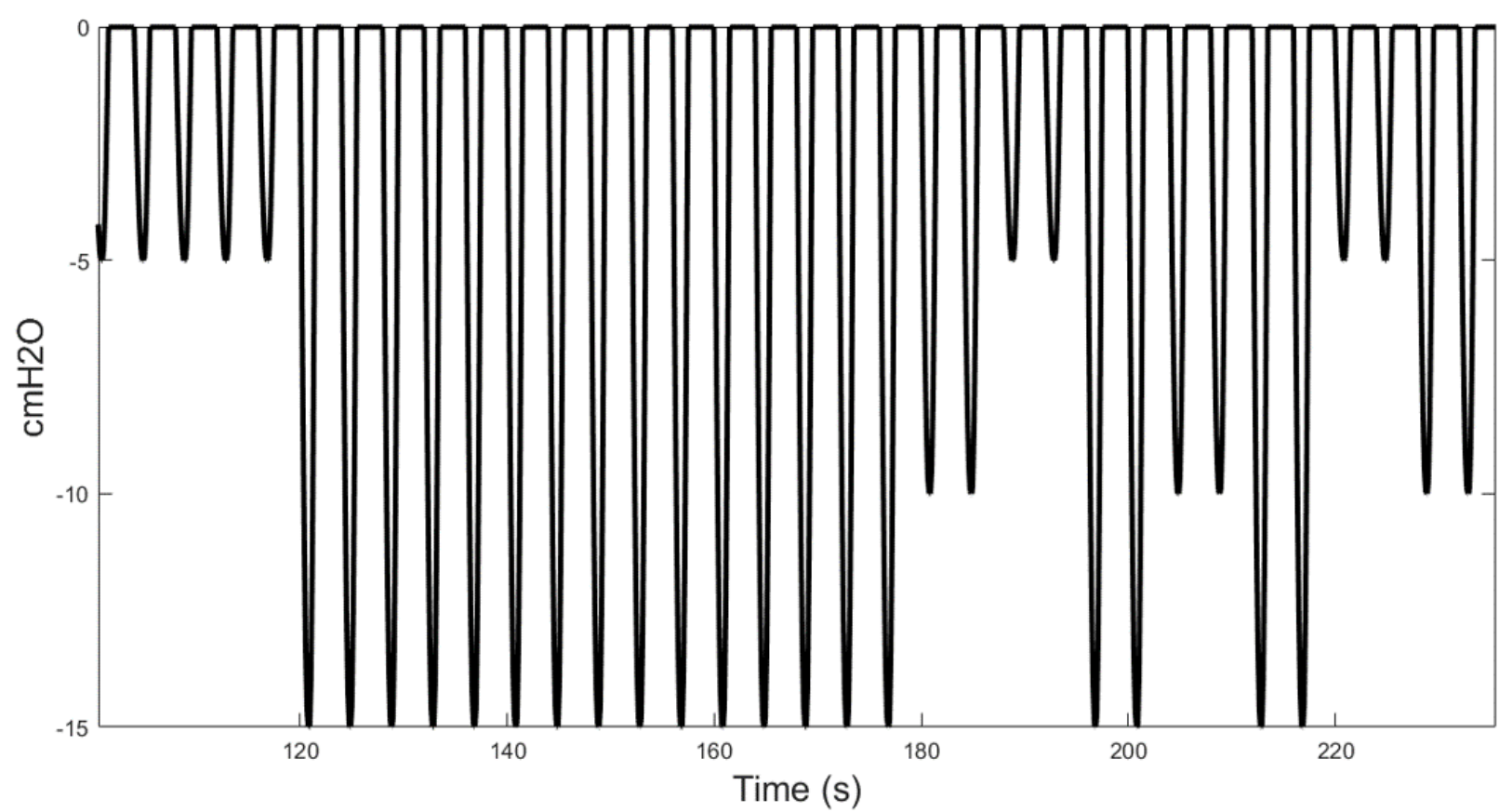

Figure 4.7. The "clean" Peso signal simulated from the ASL 5000 to illustrate Peso Signal \#9 from Table 6. The max pressure amplitude of $\Delta \mathrm{P}$ was varied between -5 to $-15 \mathrm{cmH}_{2} \mathrm{O}$. 


\section{Processing of ASL 5000 Peso Signals}

Although acquisition of the ASL 5000 Peso signals is an important step, processing of the data is necessary to obtain the final "clean" Peso use for FrankEinstein's Peso signals.

A noticeable characteristic of the Peso signals obtained from the ASL 5000 is the sharp inflection at the transition points between two phases: i) the end of expiratory hold and the beginning of inspiratory increase, and ii) the end of expiratory release and the beginning of expiratory hold. The sharp inflection points at the two transitions points are indicated by blue arrows in Figure 4.6. After consulting with the clinical collaborators, it was agreed that the sharp inflection points at those transitions are not characteristic of Peso signals from humans. In fact, the inflection points should have a gradual slope, and therefore a smoother Peso morphology is necessary. To smoothen the signal and remove the sharp inflection points, a 70-point moving average filter was applied to the ASL 5000 Peso signal.

Another important aspect of the Peso signals was that they were acquired at $256 \mathrm{~Hz}$. Recall that FrankEinstein's Peso signals will consist of "clean" Peso signals from the simulator and CGO extracted from real patients. To ensure consistent sampling rates with CGO data collected from humans, which will we be discussed in Section 4.3, the Peso signals from the ASL 5000 must be resampled to $200 \mathrm{~Hz}$.

Finally, normalizing the Peso signal amplitude between -1 to $1 \mathrm{cmH}_{2} \mathrm{O}$ allows us to standardize the scale of the Peso signal. Normalizing to a common scale will play an important role in later sections, because the amplitude of the extracted CGO interference varies from person to person. Through normalization, it will be possible to control the CGO interference, as well as obtain performance measurements of the proposed enhancement scheme to make direct 
comparisons without the influence of different scales. The Peso signal was normalized according to EQ4.3.

$$
\text { Peso }_{\text {Normalized }}=\frac{\text { Peso }}{\max (\text { abs }(\text { Peso }))} \quad(\text { EQ4.3 })
$$

Figure 4.8 shows the steps outlined in this section and their associated Peso traces. An example of the final "clean" Peso signal is shown at the normalization step. 

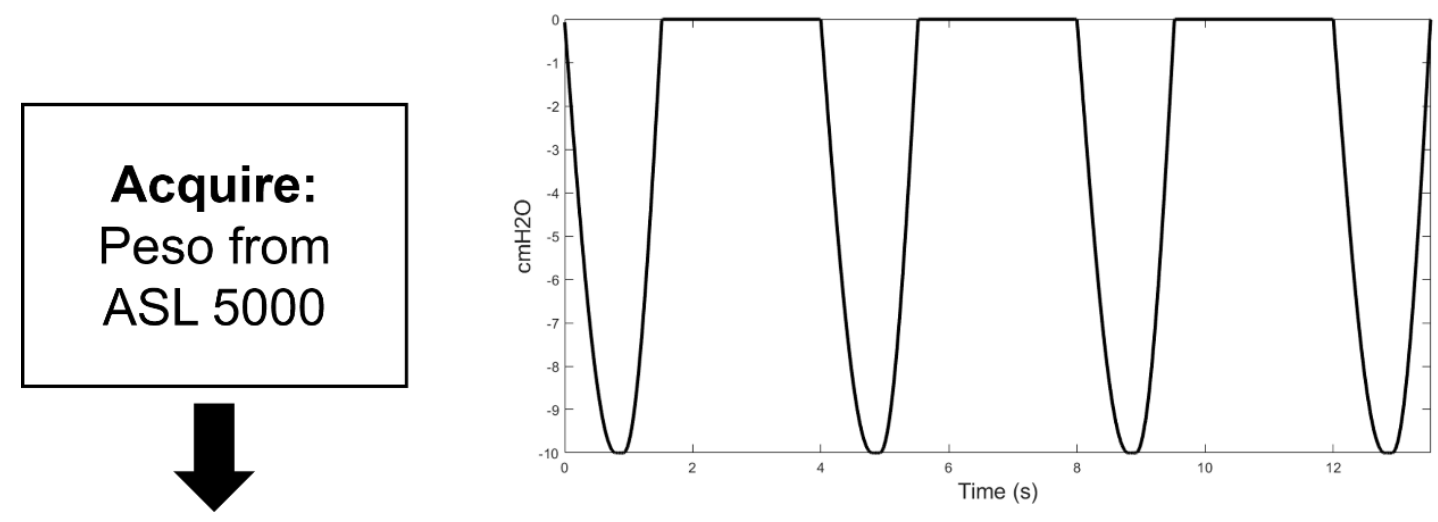

Resample:

$256 \mathrm{~Hz}$ to

$250 \mathrm{~Hz}$
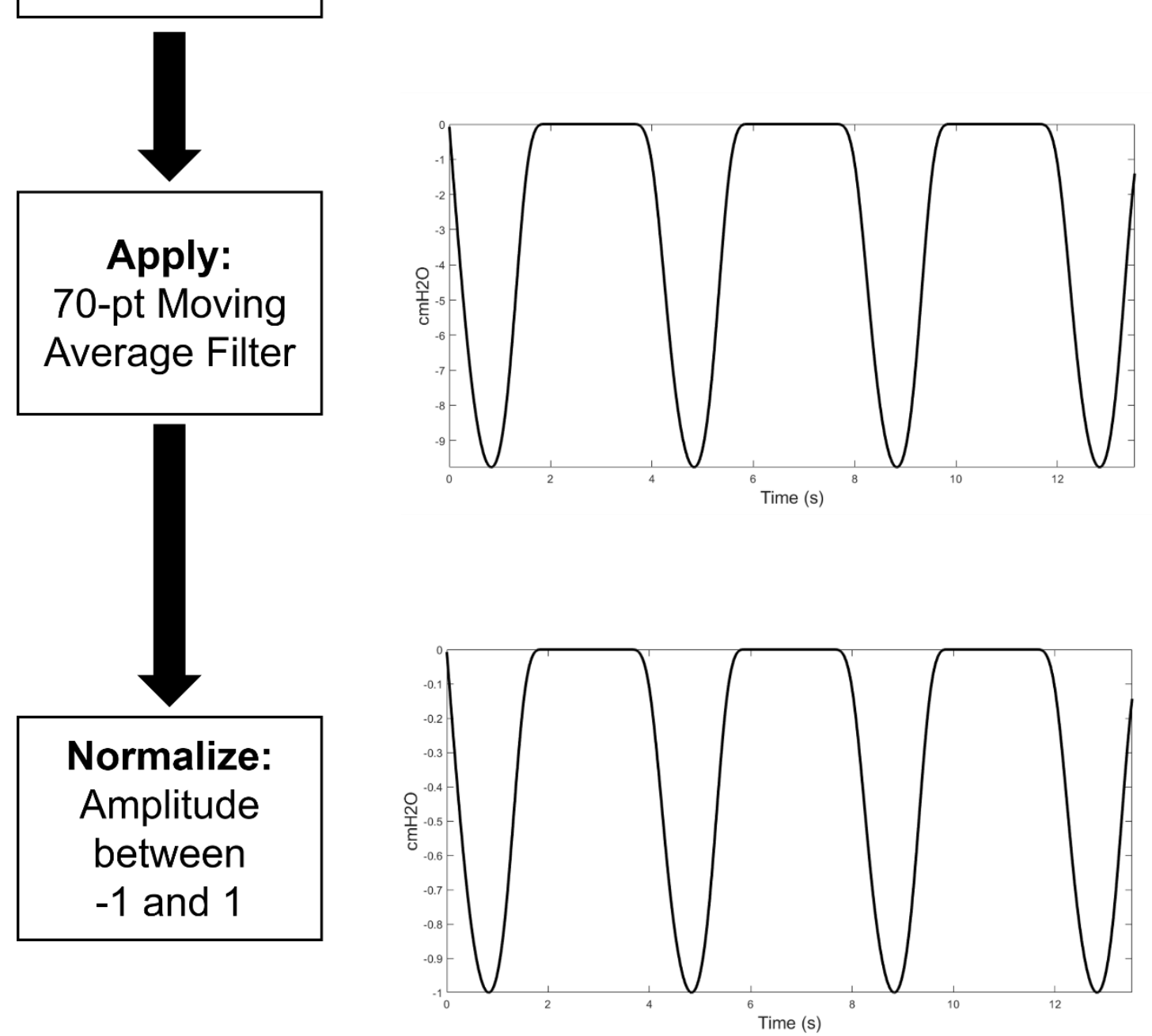

Figure 4.8. The workflow used to generate the final “clean” Peso for FrankEinstein's Peso signal. 


\subsection{Extracting CGO Interference}

In Section 4.2, the process used to obtain FrankEinstein's "clean" Peso signal was described, which plays an important role as the reference signal used for benchmarking and quantifying the performance of the proposed enhancement scheme. The counterpart to the reference signal is the interference source, which ideally, should be controllable. The purpose of controlling the interference source, which in this case is the CGOs, is to test the operating limits of the proposed enhancement scheme. The aim of this section is to describe how the CGO interference was derived from patients in the ICU.

\subsubsection{Dataset}

The dataset used in this section is an expansion of Dataset \#2 described in Chapter 3. This dataset now contains 25 different patients under three different breathing conditions: i) passive breathing, ii) spontaneous breathing with PSV, and iii) breathing with a t-piece. Thus, the dataset contains 75 signals in total.

Recall that passive breathing suppressed the patients' respiratory drive by inducing muscle paralysis to their respiratory muscles, while spontaneous breathing with PSV is a form of active breathing, which gives patients an opportunity to take on more of the ventilatory. The third condition occurs when the patients were breathing using a t-piece and is considered another subset of active breathing. When breathing using a t-piece, patients were provided an external oxygen supply but were temporarily disconnected from the mechanical ventilator. Like in spontaneous breathing with PSV, breathing with a t-piece also encourages patients to take on a greater proportion of the ventilatory effort. The active breathing conditions described in this section are commonly methods of weaning patients off mechanical ventilation in the ICU. This dataset 
contains five types of data: i) Airway Pressure $\left[\mathrm{cmH}_{2} \mathrm{O}\right]$, ii) Esophageal Pressure $\left[\mathrm{cmH}_{2} \mathrm{O}\right]$, iii) Gastric Pressure $\left[\mathrm{cmH}_{2} \mathrm{O}\right]$, iv) Flow [L/s], and v) Transpulmonary Pressure $\left[\mathrm{cmH}_{2} \mathrm{O}\right]$. The signals were sampled at $200 \mathrm{~Hz}$, and the duration of the signals range between 50.92 seconds to 644.86 seconds. The collection of this data was approved by St. Michael's Hospital's Research Ethics Board under REB\# 15-369. A summary of the data used in this section is shown in Table 7.

TABLE 7: SUMmaRY OF THE DATASET USED FOR EXTRACTING CGOS FOR FRANKEINSTEIN's PESO SIGNAL.

\begin{tabular}{|c|c|c|c|c|c|c|c|}
\hline $\begin{array}{c}\text { Dataset } \\
\#\end{array}$ & $\begin{array}{c}\text { REB } \\
\#\end{array}$ & $\begin{array}{l}\text { No. of } \\
\text { Patients }\end{array}$ & $\begin{array}{l}\text { Condition of } \\
\text { Patients }\end{array}$ & Types of Data & $\begin{array}{l}\text { Sampling } \\
\text { Frequency } \\
\text { (Hz) }\end{array}$ & $\begin{array}{l}\text { Duration } \\
\text { of } \\
\text { Signals } \\
\text { (sec) }\end{array}$ & $\begin{array}{c}\text { Total } \\
\text { No. of } \\
\text { Signals }\end{array}$ \\
\hline 3 & $\begin{array}{l}15- \\
369\end{array}$ & 25 & $\begin{array}{l}\text { 1. Passive } \\
\text { 2. Spontaneous } \\
\text { with PSV } \\
\text { 3. Breathing } \\
\text { with t-piece }\end{array}$ & $\begin{array}{l}\text { 1. Airway Pressure } \\
\text { 2. Esophageal } \\
\text { Pressure } \\
\text { 3. Gastric Pressure } \\
\text { 4. Flow } \\
\text { 5. Transpulmonary } \\
\text { Pressure }\end{array}$ & 200 & $\begin{array}{l}50.92- \\
644.86\end{array}$ & 75 \\
\hline
\end{tabular}

\subsubsection{Setup for CGO Extraction}

In Chapter 3, it was shown that while the proposed enhancement scheme is capable of suppressing CGO, it is also able to extract CGO. Based on those experiments, the EEMD algorithm was applied to Peso signals from patients in Dataset \#3 to generate the IMFs and reconstruct a CGO signal. The number of ensemble trials used in the decomposition was fixed at 100 and an amplitude of white noise computed using EQ4.4 was added into each trial.

$$
n=0.2 \times \operatorname{std}(\text { Peso }) \quad(\text { EQ4.4) }
$$

Based on face validation with collaborating clinicians, the IMFs relevant to the CGO process were manually selected and summed together to reconstruct the CGO interference. 
Finally, the extracted CGO was normalized between -1 and 1 according to EQ4.5:

$$
C G O_{\text {Normalized }}=\frac{C G O}{\max (a b s(C G O))}
$$

As mentioned in Section 4.2, normalizing the amplitude values of CGO between -1 and 1 is important when making direct comparisons especially since the CGO is extracted from different patients. Using normalized CGOs also ensures that the interference in the FrankEinstein "noisy" Peso signals can be controlled, which is ultimately useful in obtaining the operating limits of the proposed enhancement scheme. The workflow used to extract the CGO interference is shown in Figure 4.9.

From the 75 signals in Dataset \#3, 20 were randomly selected and decomposed using EEMD. After applying the workflow shown in Figure 4.9, 20 CGOs were extracted in total to act as controllable interferences for FrankEinstein's "noisy” Peso signals. It was found that the heart rate of the 20 CGOs range between $54 \mathrm{bpm}$ to $144 \mathrm{bpm}$.

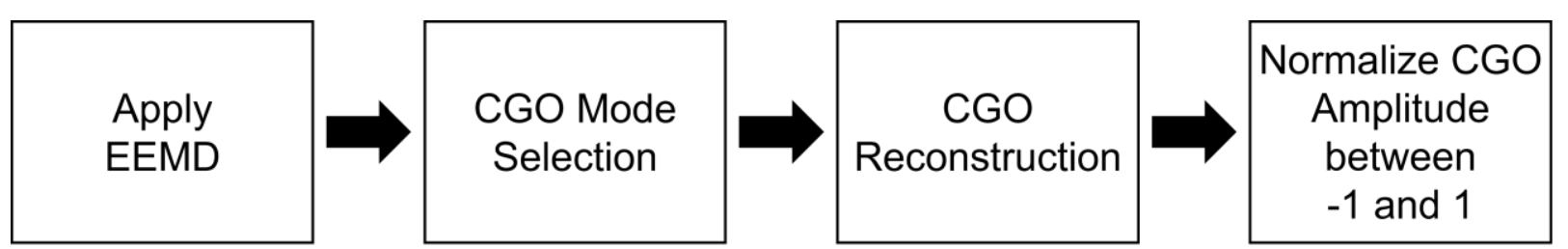

Figure 4.9. The workflow used to extract the CGO interference from 20 randomly selected patients in Dataset \#3.

\subsubsection{Extracting the CGO Signals}

The first step in CGO extraction is the application of EEMD to one of the 20 Peso signals from Dataset \#3 to obtain the IMFs. The IMFs for one of the Peso signals captured during passive breathing is shown in Figure 4.10. For the purposes of clarity, note that only the first eight IMFs out of 16 for this specific signal were shown and visualized. The rationale behind this decision 
was that the CGO would only appear in the first couple of IMFs. Additionally, the EEMD algorithm was applied to the entire duration of the $\sim 368$ second signal. However, only the first 30 seconds are shown in Figure 4.10 in order to clearly visualize the traces in each of the IMFs.
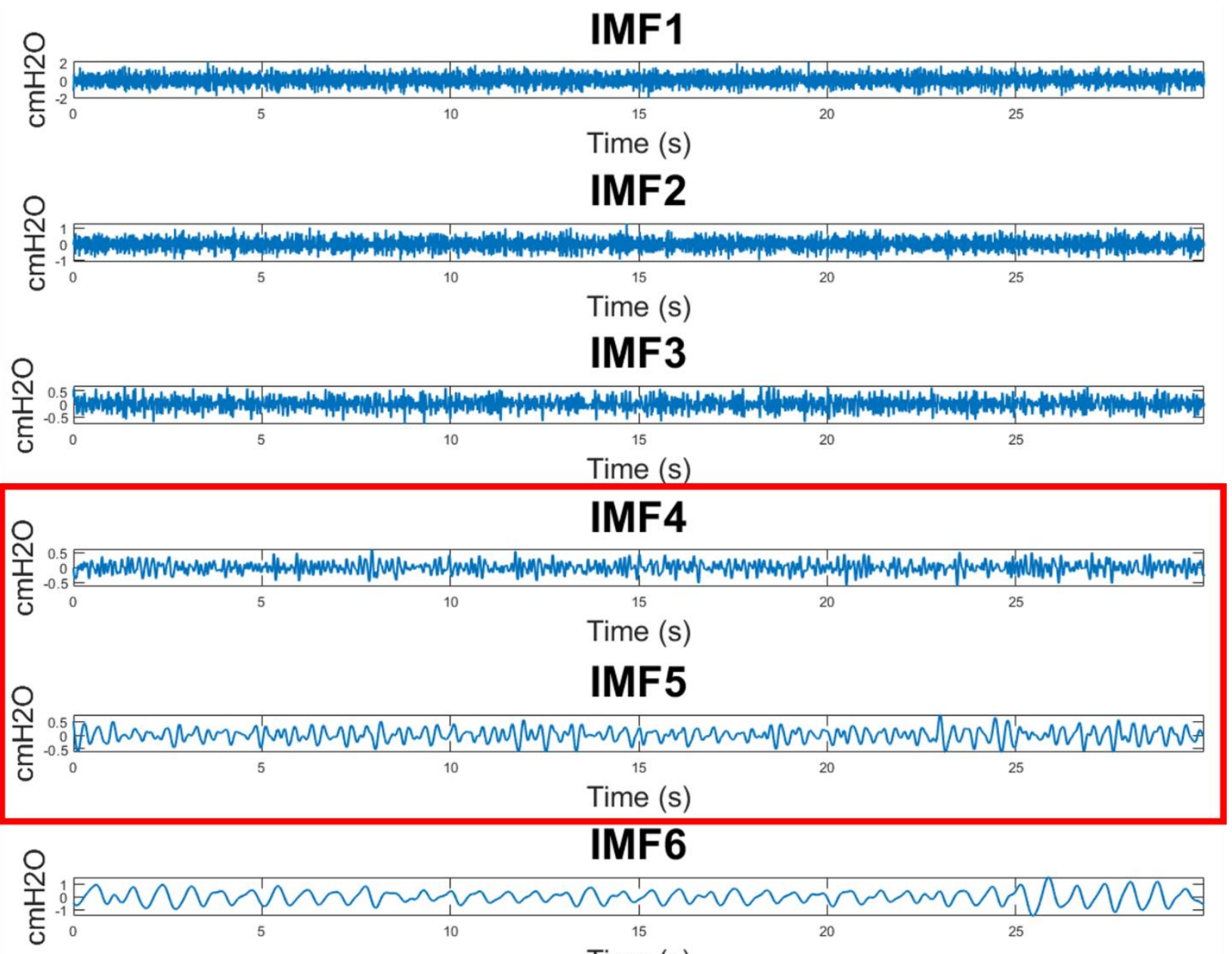

Time (s)

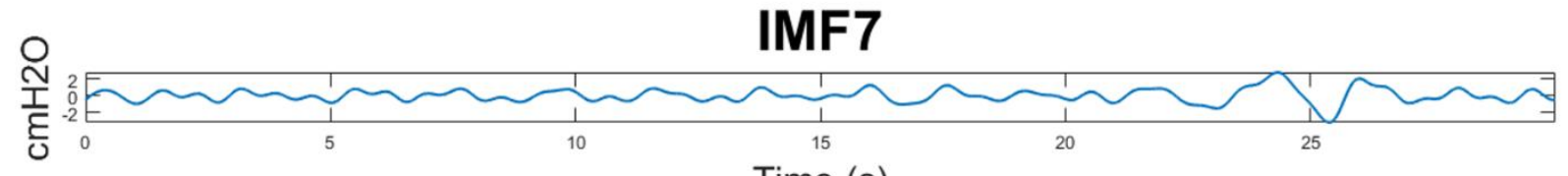

Time (s)

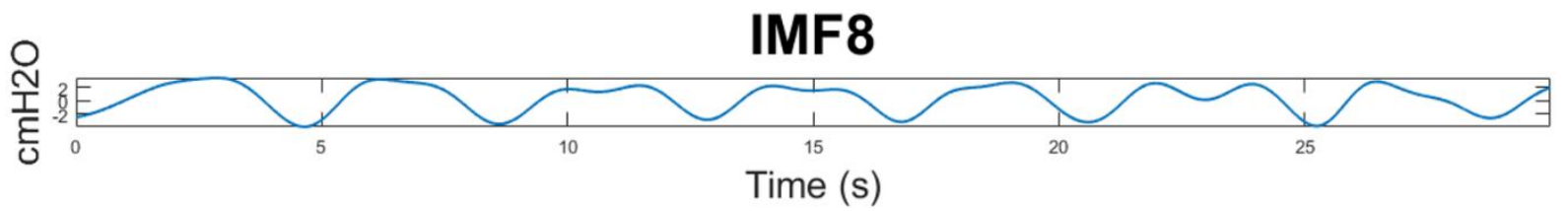

Figure 4.10. The first eight IMFs of a passively breathing patient's Peso. The IMFs related to CGO are indicated by a red box. 
The second and third step of the workflow is to select the relevant modes and reconstruct the CGO signal. From Figure 4.10, IMFs 4 and 5 were selected and deemed to best represent the CGO signal. The time-domain and frequency-domain representations of the raw Peso signal and the reconstructed CGO are shown in Figure 4.11. From the time-domain representation, the morphology of the reconstructed CGO (shown in red) appears to look very similar to the CGO present in the raw Peso (shown in black) observed at end-expiratory pressure (i.e. the plateau occurring at the end of expiration). The magnitude spectrum also shows that the reconstruction has been able to retain the majority of the CGO's fundamental frequency relative to the raw Peso's magnitude spectrum, as well as the CGO's harmonics. Although some of the fundamental frequency of the CGO was lost in the reconstruction, the task of selecting the IMF combination was a balance between reconstructing a "clean" CGO morphology and maintaining as much CGO frequency components. Other IMF combinations were tested as well; some combinations were able to retain all the CGO frequency content but at the expense of including more high-frequency noise in the time-domain representation. Alternatively, choosing IMFs to reconstruct a much “cleaner" CGO would result in not only a loss of significant frequency content, but may even lead to a loss of morphological characteristics or important "landmarks" such as the notches.

The final step in extracting FrankEinstein's CGO signal is to normalize the amplitude of the reconstructed CGOs between -1 and $1 \mathrm{cmH}_{2} \mathrm{O}$. The CGO trace used as an example in this section, which has also been used to construct one of FrankEinstein's "noisy" Peso signal, is shown in Figure 4.12. 


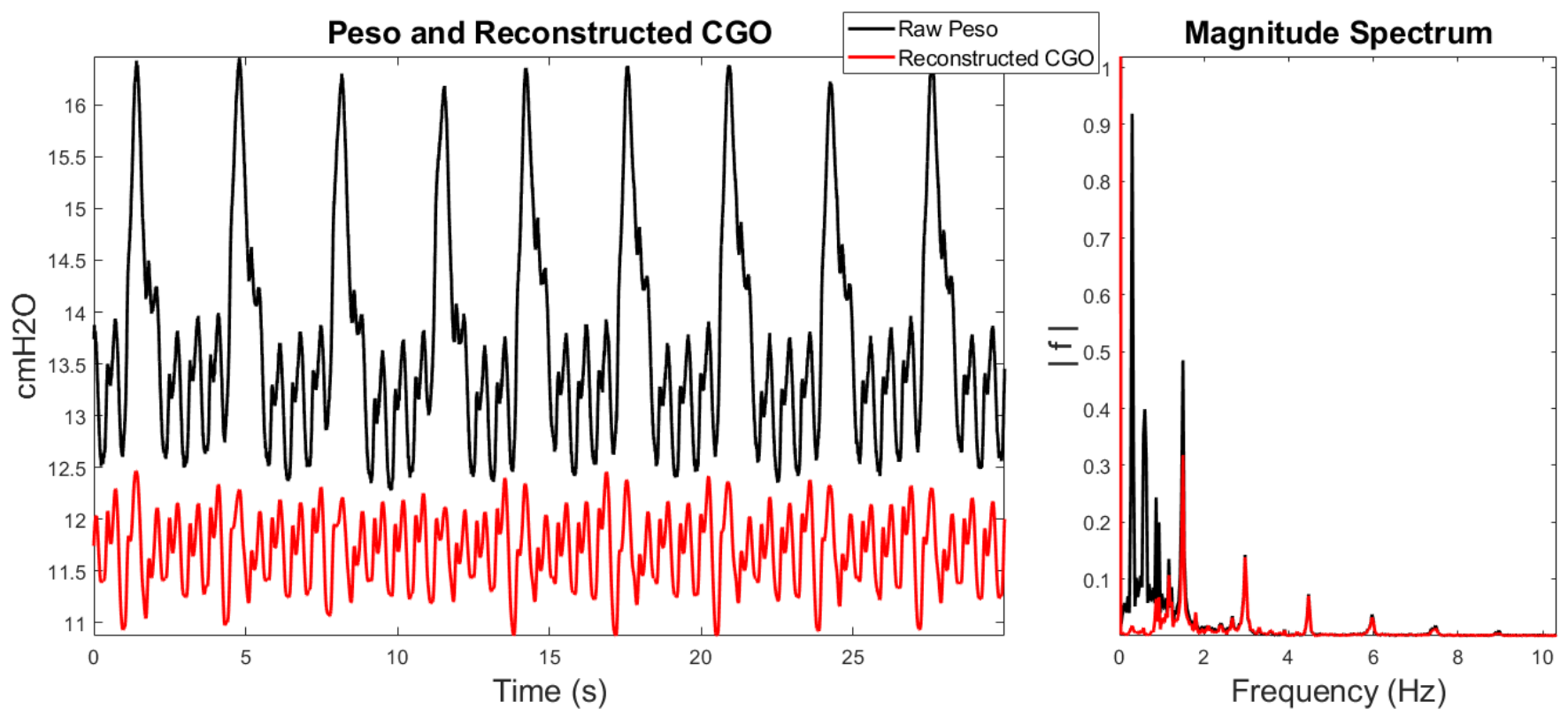

Figure 4.11. The time-domain representation of the Raw Peso (black) and the reconstructed CGO using IMFs 4 to 5 (red) is shown on the right.

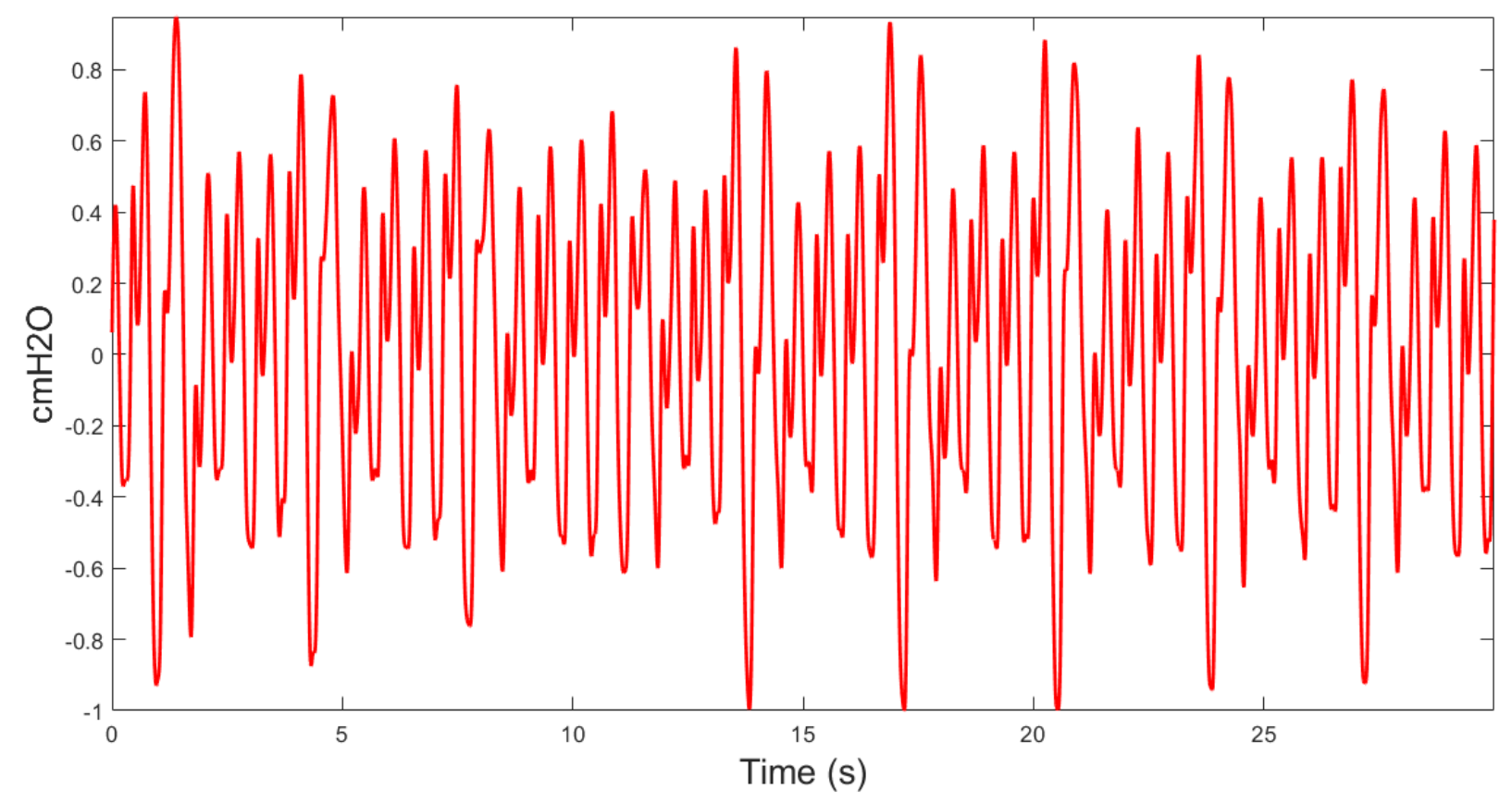

Figure 4.12. The normalized CGO that was extracted from a passively breathing patient. 


\section{4 "Reanimating” FrankEinstein's "Noisy" Peso Signal}

In Sections 4.2 and 4.3, the methodologies used to generate the Peso and extract the CGO signals were described. To create FrankEinstein's "noisy" Peso signal, the two components are added together according to EQ4.1. However, as discussed throughout this Chapter, both the Peso and CGO amplitudes were normalized between -1 and 1 to ensure that direct comparisons can be made without the influence of varying scales. The normalization of the CGO amplitude plays an important role in finding the operating limits of the proposed Peso enhancement scheme; by normalizing the CGO signal, it is possible to control the level of interference in a given FrankEinstein Peso signal by applying a scaling factor to the CGO. A visual representation of the process used to create FrankEinstein's Peso signal is shown in the block diagram of Figure 4.13.

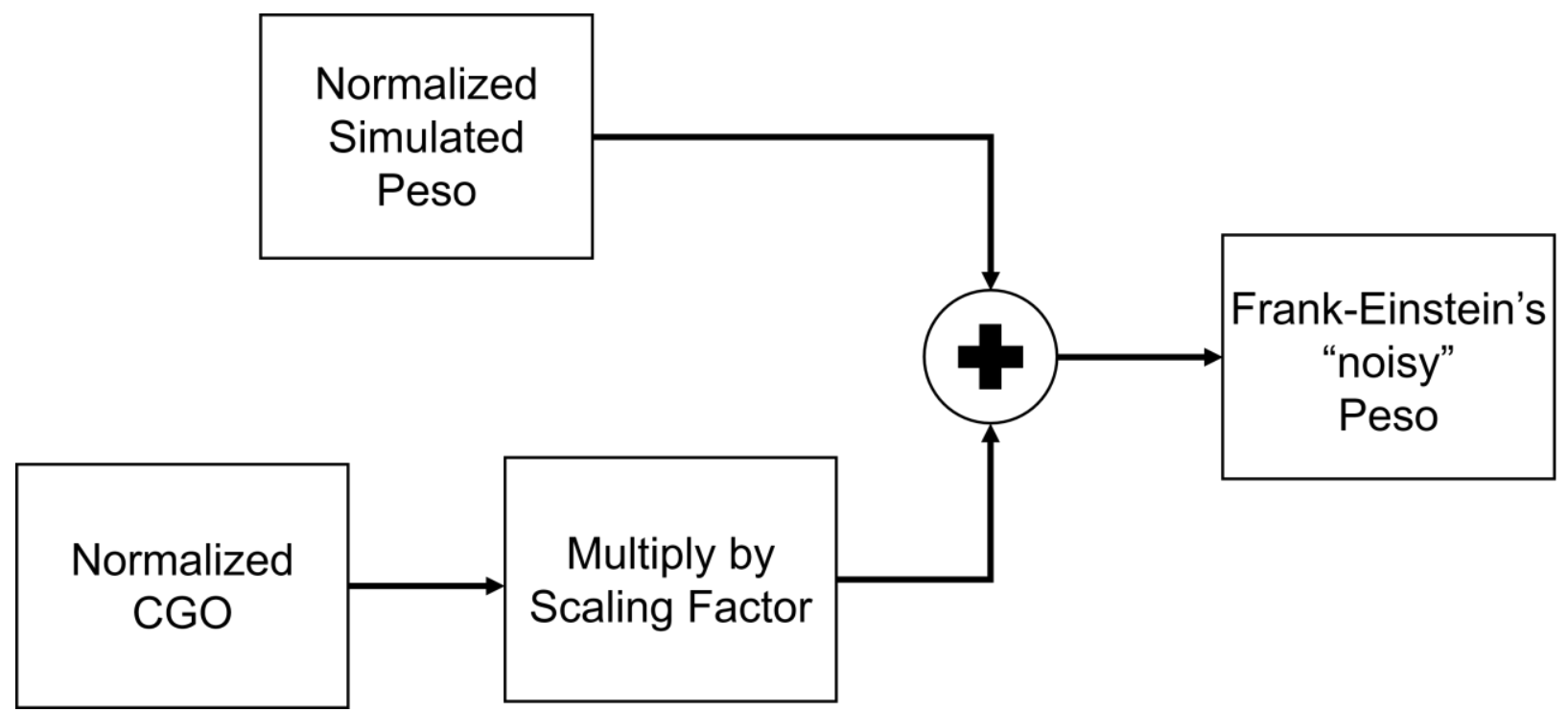

Figure 4.13. The process of creating FrankEinstein's “noisy” Peso signal. 
To simulate different levels of CGO interference, 10 scaling factors were applied to each of the 20 extracted CGOs. The 10 scaling factors range between $0.1 \mathrm{cmH}_{2} \mathrm{O}$ to $1 \mathrm{cmH}_{2} \mathrm{O}$ with increments of $0.1 \mathrm{cmH}_{2} \mathrm{O}$. The 10 Peso signals shown in Figure 4.14 is an example of one FrankEinstein "noisy" Peso signal with the 10 different levels of CGO interference.

In generating FrankEinstein's "noisy" Peso signal, each of the 20 CGO signals were scaled 10 different times as described previously, which resulted in 200 CGO signals. The 200 CGO signals were then superimposed onto each of the nine simulated Peso signals obtained in Section 4.2. Through this process, a total of 1800 "noisy" Peso signals were created for FrankEinstein's Peso dataset. Table 8 summarizes the properties of FrankEinstein's “Noisy” Peso dataset.

TABle 8: A SUMmary OF FrankEINSTEIN'S “Noisy” Peso Signals DATASET.

\begin{tabular}{|c|c|c|c|c|c|c|}
\hline Peso Signal \# & $\begin{array}{c}\text { Respiratory } \\
\text { Rate } \\
\text { (breaths } / \text { min) }\end{array}$ & $\begin{array}{c}\operatorname{Max} \Delta P \\
\left(\operatorname{cmH}_{2} \mathrm{O}\right)\end{array}$ & $\begin{array}{l}\text { Total No. } \\
\text { of CGO } \\
\text { Signals }\end{array}$ & $\begin{array}{c}\text { Heart } \\
\text { Rate } \\
\text { (bpm) }\end{array}$ & $\begin{array}{l}\text { No. of } \\
\text { Scale } \\
\text { Factors }\end{array}$ & $\begin{array}{c}\text { Total } \\
\text { No. of } \\
\text { scaled } \\
\text { CGO } \\
\text { Signals }\end{array}$ \\
\hline 1 & 15 & -10 & \multirow{9}{*}{20} & \multirow{9}{*}{$\begin{array}{l}54- \\
144\end{array}$} & \multirow{9}{*}{10} & \multirow{9}{*}{200} \\
\hline 2 & 17 & -10 & & & & \\
\hline 3 & 19 & -10 & & & & \\
\hline 4 & 21 & -10 & & & & \\
\hline 5 & 23 & -10 & & & & \\
\hline 6 & 27 & -10 & & & & \\
\hline 7 & 29 & -10 & & & & \\
\hline 8 & 31 & -10 & & & & \\
\hline 9 & 15 & -5 to -15 & & & & \\
\hline $\begin{array}{l}\text { Total No. of } \\
\text { "Noisy" } \\
\text { Signals }\end{array}$ & \multicolumn{6}{|c|}{1800} \\
\hline $\begin{array}{c}\text { Sampling } \\
\text { Frequency } \\
(\mathrm{Hz})\end{array}$ & \multicolumn{6}{|c|}{200} \\
\hline
\end{tabular}



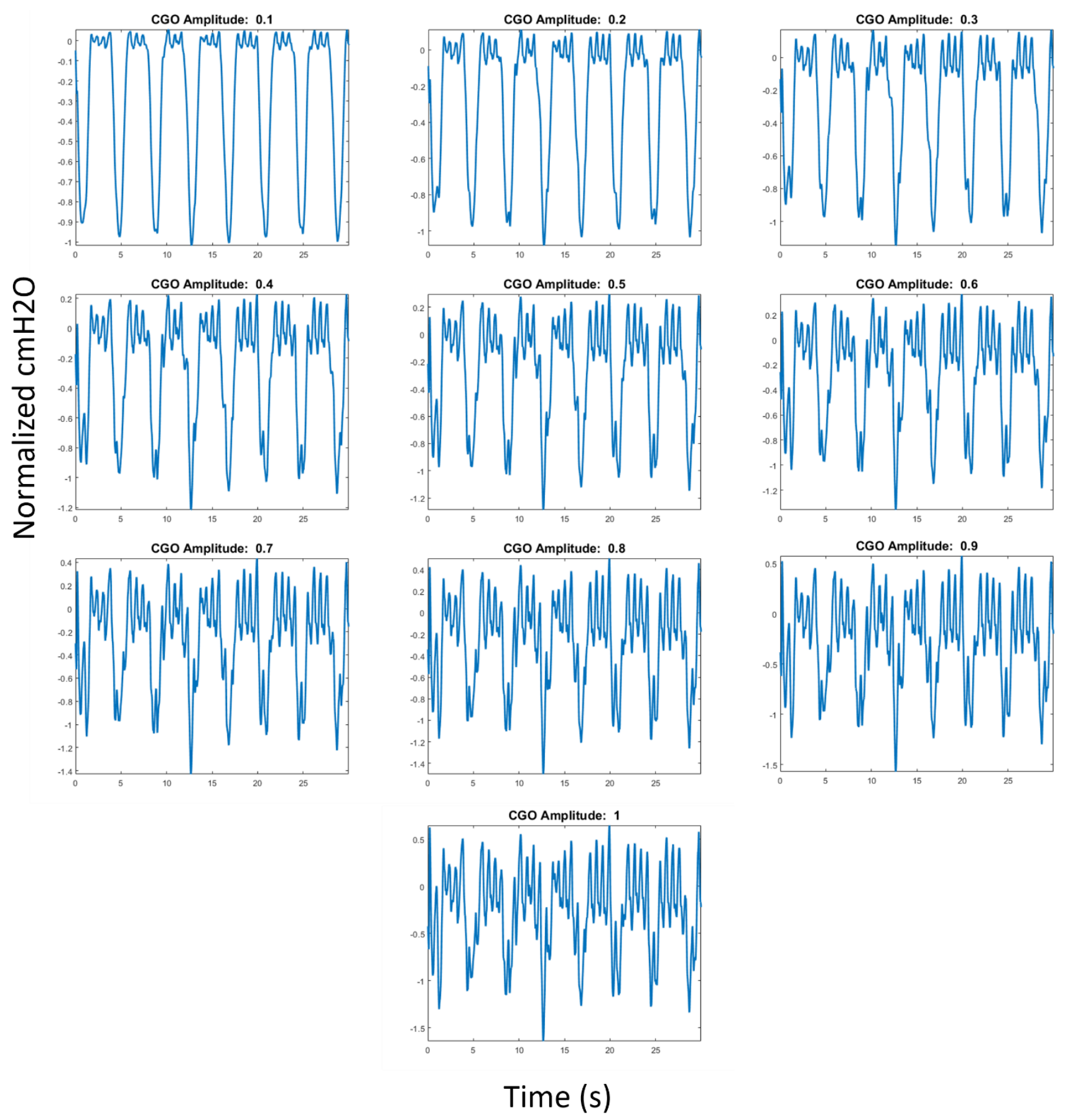

Figure 4.14. A set of FrankEinstein Peso signals using one CGO that was scaled 10 different times. 


\subsection{Applying the Proposed Enhancement Scheme to FrankEinstein's "Noisy"}

\section{Peso Signal}

Over the course of this Chapter, the focus has been on the methods used to create FrankEinstein's "Noisy" Peso signal. As mentioned back in Section 4.1, the motivation behind creating the FrankEinstein Peso signals was to overcome the challenges associated with subjective assessments of the proposed enhancement scheme's performance. Because the base Peso signals were synthetically generated using the ASL 5000, a reference signal is available for the purposes of objectively quantifying the enhancement performance. Moreover, the amplitude of the CGO signals that comprise FrankEinstein's "noisy” Peso can be adjusted using a scale factor. By scaling the CGO amplitude, the level of interference present in the Peso signal can be controlled, and the upper operating limit of the proposed enhancement scheme can be determined with respect to the signal-to-interference ratio (SIR). Thus, the aim of this section is to apply the proposed enhancement scheme to the FrankEinstein Peso dataset, and quantify its performance using a popular distortion measure known as percentage root mean square difference (PRD).

\subsubsection{Dataset}

The dataset used in this section is FrankEinstein's “noisy” Peso signals. FrankEinstein's Peso signals were created to mimic spontaneously/actively breathing patients with varying levels of CGO interference. Nine "clean" Peso signals were simulated on the ASL 5000 and used as the basis for FrankEinstein's Peso. The properties of the Peso signals were previously described in Section 4.2 and are summarized in Table 6. In addition to the Peso, 20 different CGO signals were extracted from real ICU patients. Each of the 20 CGOs were scaled using 10 factors and superimposed onto the Peso signals. The scaling factors range between 0.1 to 1 with 0.1 
increments. The heart rate from the 20 randomly selected CGOs range between $54 \mathrm{bpm}$ to 144 bpm.

In total, 1800 "noisy" Peso signals were created for FrankEinstein's Peso dataset. The signals have a sampling frequency of $200 \mathrm{~Hz}$ and range between 55 to $\sim 589$ seconds in duration. To make direct performance comparisons, the data can be divided according to the 10 scaling factors. For each of the 10 factors, there are 180 different "noisy" Peso signals. A summary of the components comprising FrankEinstein's dataset is shown in Table 9.

TABLE 9: A SUMMARY OF FRANKEINSTEIN'S DATASET.

\begin{tabular}{|c|c|c|c|c|c|}
\hline $\begin{array}{c}\text { Dataset } \\
\quad \#\end{array}$ & $\begin{array}{l}\text { Simulated } \\
\text { Condition }\end{array}$ & Types of Data & $\begin{array}{l}\text { Total No. of } \\
\text { Signals }\end{array}$ & $\begin{array}{l}\text { Sampling } \\
\text { Frequency } \\
\quad(\mathbf{H z})\end{array}$ & $\begin{array}{c}\text { Duration } \\
\text { of } \\
\text { Signals } \\
\text { (sec) }\end{array}$ \\
\hline 4 & $\begin{array}{l}\text { Spontaneous/Active } \\
\text { Breathing }\end{array}$ & $\begin{array}{l}\text { FrankEinstein's } \\
\text { "Noisy" Peso }\end{array}$ & 1800 & 200 & $\begin{array}{c}55- \\
589.24\end{array}$ \\
\hline $\begin{array}{l}\text { No. of } \\
\text { CGO }\end{array}$ & \multicolumn{2}{|l|}{20} & $\begin{array}{c}\text { No. of ASL 500 } \\
\text { "Clean" Peso }\end{array}$ & \multicolumn{2}{|c|}{9} \\
\hline $\begin{array}{l}\text { No. of } \\
\text { CGO } \\
\text { Scale } \\
\text { Factors } \\
\end{array}$ & \multicolumn{2}{|c|}{$\begin{array}{c}10 \\
\text { (0.1 to } 1 \text { with an increment of } 0.1 \text { ) }\end{array}$} & $\begin{array}{c}\text { No. of } \\
\text { FrankEinstein } \\
\text { Peso per Scale } \\
\text { Factor } \\
\end{array}$ & \multicolumn{2}{|c|}{180} \\
\hline
\end{tabular}

\subsubsection{Experimental Setup}

The proposed enhancement scheme using the EEMD algorithm will be applied to FrankEinstein's Peso dataset using the same parameters established in Chapter 3. The number of ensemble trials is fixed at 100, and the amplitude of white noise is calculated according to EQ4.6:

$$
n=0.2 \times \operatorname{std}\left(\text { Peso }_{\text {Frank-Einstein,noisy }}\right) \quad \text { (EQ4.6) }
$$




\section{IMF Selection}

Although the proposed enhancement scheme was successfully used in Chapter 3 to suppress CGO from the Peso signal, one of the major limitations associated with the technique is the manual selection of relevant IMFs. In the previous Chapter, the size of the datasets was quite small ( 2 signals for passive breathing and 6 signals for spontaneous breathing), which would allow for the clinical collaborators to visually inspect and select the IMFs. However, manual IMF selection by domain experts does not scale up well; it is not feasible to have experts perform IMF selection on significantly larger datasets such as FrankEinstein's Peso dataset, which contains 1800 different "noisy" Peso signals. To overcome the limitations imposed by manual selection, an automatic IMF selection threshold criterion of $0.75 \mathrm{~Hz}$ was defined. The rationale behind the IMF selection threshold will be described later in this section. The IMF selection and reconstruction process to suppress CGO for a Peso signal with $M$ number of IMFs using the proposed threshold criterion is implemented as follows:

1. Beginning with $N=1$, generate the magnitude spectrum for IMF $N$ using the FT.

2. Find the maximum peak and its corresponding frequency bin, $f$.

3. If $f \geq 0.75 \mathrm{~Hz}$, the IMF is representative of either high-frequency noise or CGO. Go to the next IMF (i.e. $N=N+1$ ) and iterate through steps 1 to 3 .

4. If $f<0.75 \mathrm{~Hz}$, select IMF $N$ as the starting IMF and go to step 5 .

5. Reconstruct the "clean" Peso according to EQ4.7:

$$
\text { Peso }_{\text {reconstructed }}=\sum_{i=N}^{M} I M F_{i}
$$


The IMF selection threshold criterion was inspired by the physiological characteristics derived from reported bandwidths of the heart rate. To select the threshold, a frequency value that best separates the $\mathrm{CGO} /$ heart rate and Peso spectra for ICU patients was determined. According to [42], the bandwidth of the heart rate/CGO is 0.8 to $2.5 \mathrm{~Hz}$. Alternatively, the American Heart Association has suggested that the normal adult resting heart rate ranges between $60 \mathrm{bpm}$ to 100 bpm, and can even go as low as 40 bpm for highly active individuals [60]. The aforementioned resting heart rates correspond to a bandwidth of $1 \mathrm{~Hz}$ to $1.67 \mathrm{~Hz}$ for a normal resting heart rate, and $0.67 \mathrm{~Hz}$ as the lowest possible heart rate. However, it was noted that a resting heart rate of 40 bpm was uncommon. In the clinical context, bradycardia, which is defined as an abnormally slow heart beat, can be characterized by either a heart rate less than $50 \mathrm{bpm}(<0.83 \mathrm{~Hz})$ [61] or less than $60 \mathrm{bpm}(<1 \mathrm{~Hz})$. Based on the information available about heart rates under different conditions as well as the fact that the lower bound of the CGO has been reported as $0.8 \mathrm{~Hz}$, the following threshold rule was defined: any activity greater than $0.75 \mathrm{~Hz}$ is deemed to represent CGO or highfrequency activity.

\section{Performance Measurements}

\section{$\underline{\text { Signal-to-Interference Ratio (SIR) }}$}

The SIR describes the level of interference present in the signal as a ratio of the amplitude of the signal, which in this case is the amplitude of the FrankEinstein "noisy" Peso, to the amplitude of the interference, which is the CGO. Because the CGO component of the FrankEinstein signals are controlled using 10 different scaling factors, it is possible to adjust the SIR levels. Calculating the SIR and expressing it in the decibel scale is accomplished using EQ4.8.

$$
S I R_{d B}=10 \log _{10}\left(\frac{A_{\text {Peso,Frank-Einstein }}}{A_{C G O}}\right)^{2}
$$




\section{$\underline{\text { Percentage Root Mean Square Difference (PRD) }}$}

The PRD is a distortion measure that is widely used to quantify the amount of information lost as a percentage during ECG compression and reconstruction. In the context of this work, the proposed enhancement scheme reconstructs a "clean" version of the Peso signal by removing CGO information. While suppressing the information of CGOs is the aim of this research, one of the main concerns raised in Chapter $\mathbf{3}$ was the possibility of losing information from the actual Peso signal. In order to test the enhancement performance of the proposed CGO suppression technique as well as quantify how much information is lost from the Peso during reconstruction, the PRD measure is used. Since PRD value ranges have been associated with qualitative measures for ECG reconstructions based on Face Validity as shown in [62], we believe that its use is appropriate in the context of this work due to our extensive use of Face Validity. However, it is important to note that the same qualitative measures and the associated PRD ranges in [62] may not apply in this work since the signals and sources of interference are different. The definition of the PRD expressed as a percentage is shown in EQ4.9. The base Peso (i.e. "clean" or reference) signal, which was obtained from the ASL 5000 is denoted as $P e s o_{\text {simulated, }}$, while the Peso signal that was enhanced using the proposed CGO-suppression technique is represented by Peso $_{\text {reconstructed }}$.

$$
P R D=\sqrt{\frac{\sum_{n=1}^{N}\left(\text { Peso }_{\text {simulated }}(n)-\text { Peso }_{\text {reconstructed }}(n)\right)^{2}}{\sum_{n=1}^{N}\left(\text { Peso }_{\text {simulated }}(n)\right)^{2}}} \times 100 \%
$$




\section{Testing the Performance of the Proposed Enhancement Scheme}

The proposed Peso enhancement scheme using the $0.75 \mathrm{~Hz}$ threshold criterion will be applied to all 1800 of FrankEinstein's “noisy” Peso signals for IMF selection. For each of the 1800 "noisy" signals, the SIR will be determined and a corresponding PRD value for the signal reconstruction will be calculated. Insights into the performance of the CGO suppression technique will be visualized in two ways. The first will be a scatter plot of the PRD as a percentage with respect to SIR in decibels. The second will divide the FrankEinstein Peso signals according to the 10 interference amplitude scale factors. A boxplot of the PRD with respect to the 10 interference amplitudes will be created.

\subsubsection{Results and Discussions}

\section{Effects of SIR on CGO Suppression and PRD using the Proposed Enhancement Scheme}

In this work, the SIR describes the level of CGO interference present in a "noisy" Peso signal. Because the SIR is defined as the ratio of the signals power to the power of the interference, a "noisy" FrankEinstein Peso signal with high SIR contains more signal than noise. In other words, the "quality" of the signal manifests itself as a "cleaner" signal in terms of morphological characteristics. Conversely, a Peso with lower SIR means that more noise is present, and therefore visually looks like a "lower quality" signal. To draw the connection between SIR and the quality of the Peso signal's morphology, Figure 4.15 visually illustrates a FrankEinstein "noisy" Peso with an SIR of $22.8671 \mathrm{~dB}$ (CGO scale factor of 0.1) and a Peso with an SIR of 2.8671 dB (CGO scale factor of 1).

The PRD measures the error between the reference signal (i.e. the "clean" Peso) and the reconstructed signal, which was derived by suppressing the CGO from the "noisy" Peso using the 
proposed enhancement scheme. The value of PRD increases when the difference between the reference Peso and the reconstructed Peso is large. The ideal case is to have a PRD of 0\%, which would mean that all the CGO information has been removed and the morphology of the Peso trace has been completely preserved. Thus, if the PRD for a reconstructed Peso is found to be high, the morphology of the reconstructed Peso is significantly different, which would suggest that the proposed enhancement scheme would be unable to sufficiently remove the CGO.
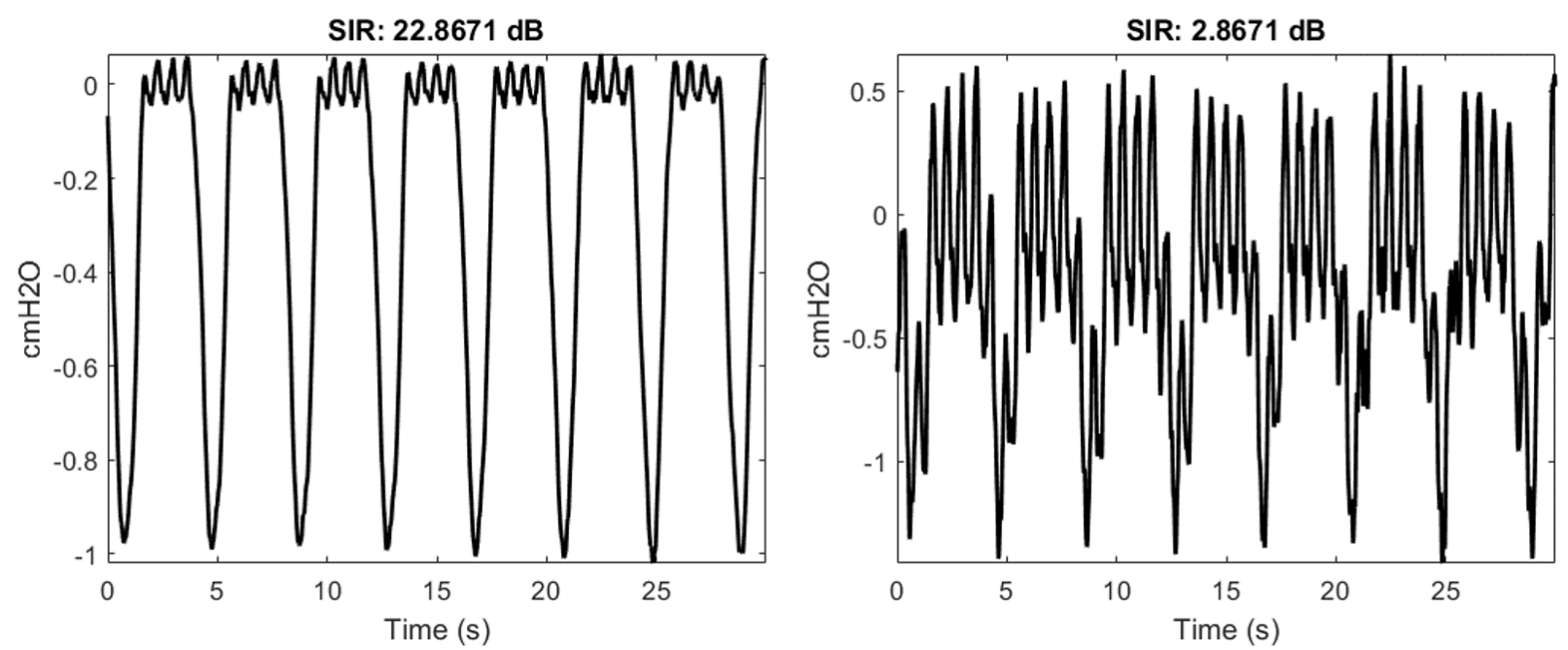

Figure 4.15. An example of "noisy" FrankEinstein Peso signals with different SIR levels. On the left is a Peso with SIR of $22.8671 \mathrm{~dB}$ and the Peso on the right has an SIR of $2.8671 \mathrm{db}$. The CGO interference is common between both signals albeit with different scaling factors. The CGO for the left Peso has a scale factor of 0.1 and the CGO for the right Peso has a scale factor of 1 .

To visually illustrate the effects of SIR on CGO suppression and Peso reconstruction, the two signals shown in Figure 4.15 were passed through the proposed enhancement scheme. For both signals, the reference Peso from the ASL 5000, the FrankEinstein "noisy" Peso, and the reconstruct Peso are shown in Figure 4.16. In Figure 4.16 (A), the FrankEinstein Peso had a CGO amplitude of $0.1 \mathrm{cmH}_{2} \mathrm{O}$, which resulted in an SIR of $22.8617 \mathrm{~dB}$. After passing the "noisy" Peso through the enhancement scheme, the reconstructed Peso has a PRD of $4.6 \%$ and is visually shown to have suppressed some of the CGO fluctuations. The FrankEinstein Peso shown in Figure 4.16 (B) has an SIR of $2.8617 \mathrm{~dB}$ as a result of a significantly larger $\mathrm{CGO}$ amplitude of $1 \mathrm{cmH}_{2} \mathrm{O}$. The 
reconstructed Peso has a PRD of $11.2 \%$, which is a relatively high distortion measure. However, from visual inspection, the reconstruction is relatively good and may be useful for clinicians. While the reconstruction for a CGO amplitude of $1 \mathrm{cmH}_{2} \mathrm{O}$ is shown to be usable for Figure 4.16 (B)'s FrankEinstein Peso, that is not always the case as demonstrated by the reconstruction in Figure 4.17. The FrankEinstein Peso of Figure 4.17 also uses the $1 \mathrm{cmH}_{2} \mathrm{O}$ scaling factor but is constructed using a different CGO signal. The SIR of this Peso is $2.01 \mathrm{~dB}$ and the PRD of the reconstruction is $53.2 \%$, which indicates that a significant amount of distortions is present. Furthermore, a visual inspection of the reconstruction confirms that the reconstructed Peso has not been able to preserve the morphology of the reference Peso. 

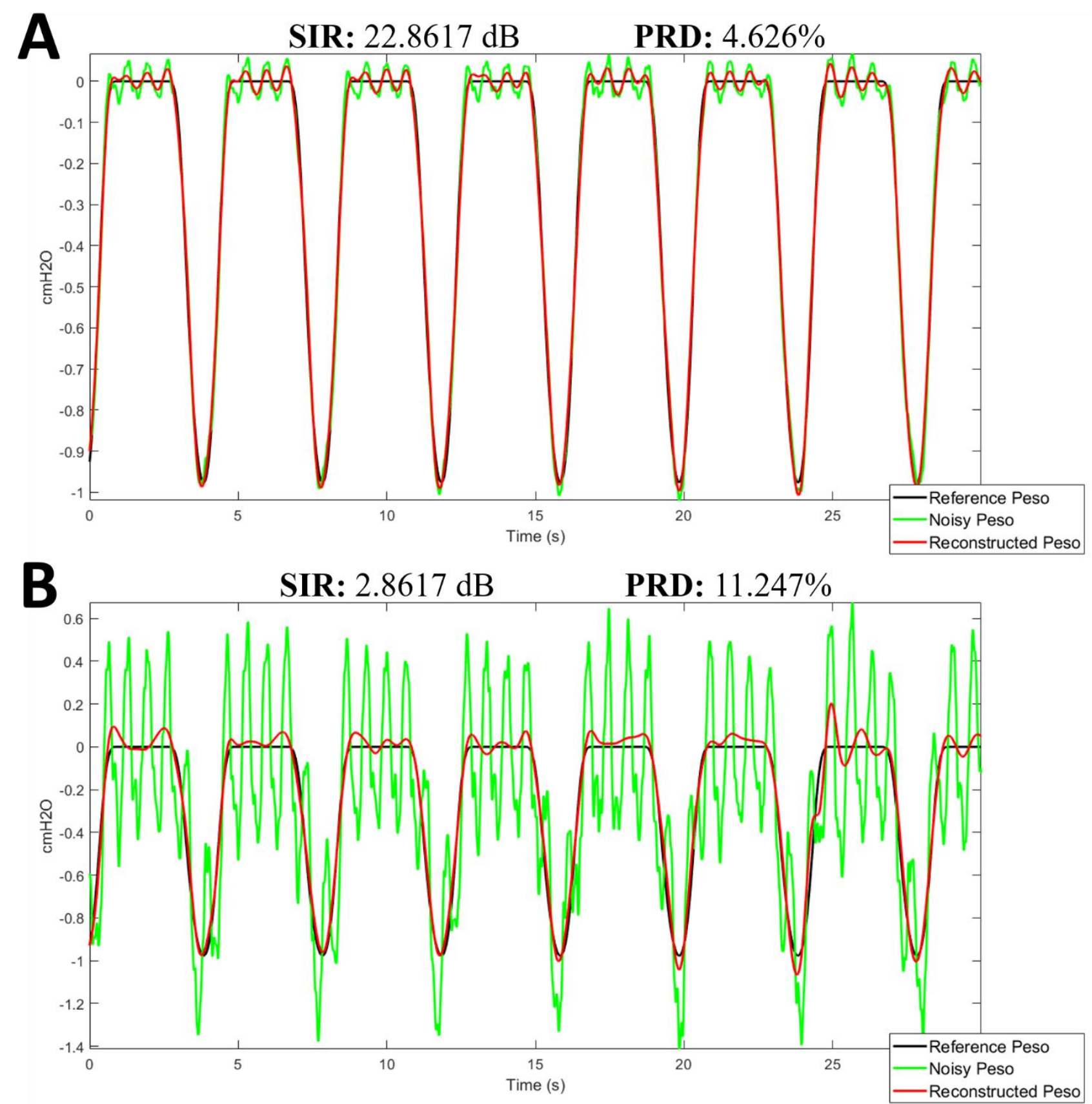

Figure 4.16. Three signals are shown in each plot: i) the reference Peso in black, which was obtained from the ASL 5000, ii) the FrankEinstein "noisy" Peso shown is in green, and iii) the reconstructed Peso resulting from the proposed enhancement scheme is in red. (A) The SIR of the FrankEinstein Peso is $22.8617 \mathrm{~dB}$. The reconstructed Peso has a PRD of 4.3\%. (B) The SIR is $2.8617 \mathrm{~dB}$, and the reconstructed Peso's PRD is $11.2 \%$. 


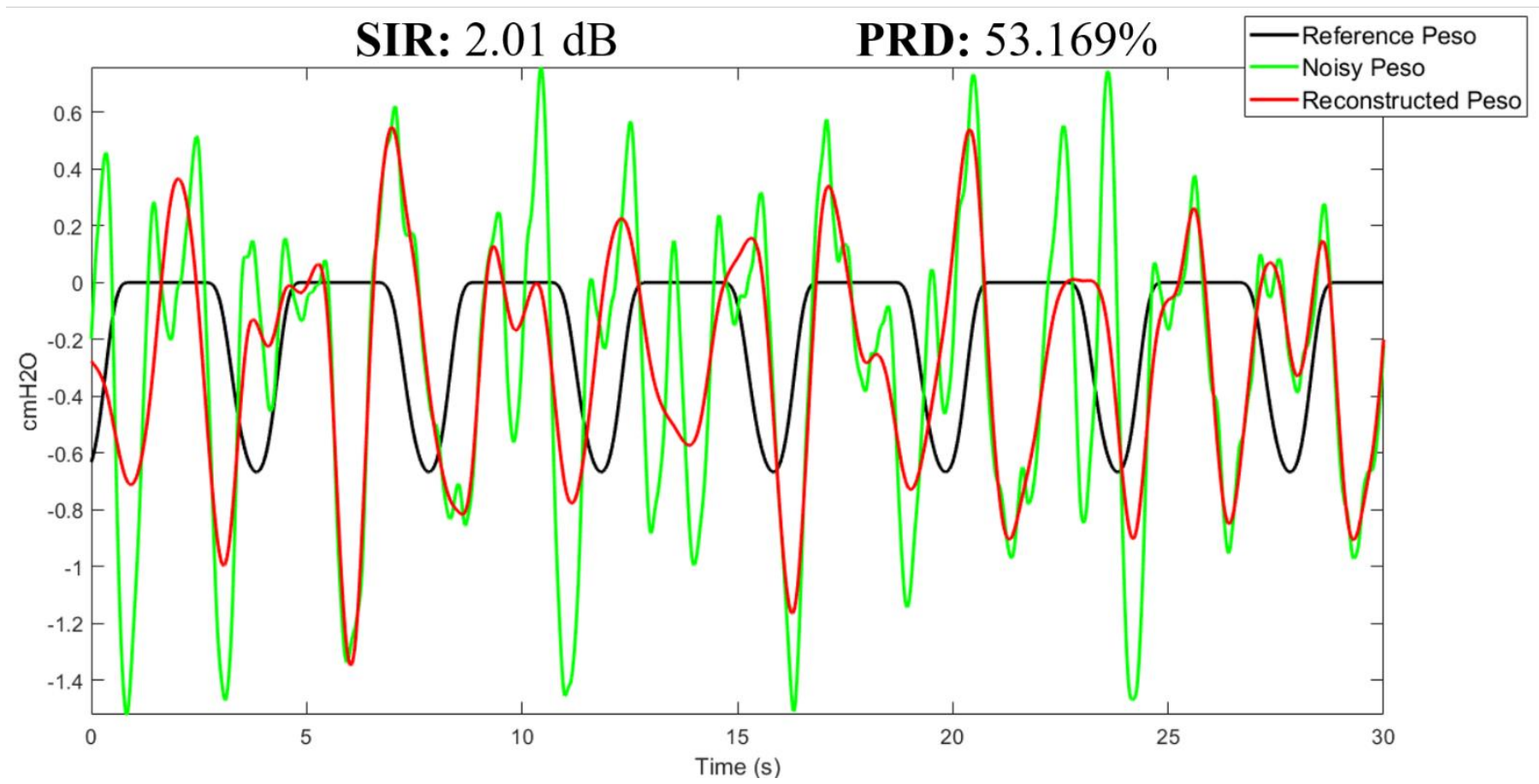

Figure 4.17. The reference Peso (black), the FrankEinstein "noisy" Peso using a CGO scaling factor of $1 \mathrm{cmH}_{2} \mathrm{O}$ (green), and the reconstructed Peso (red). It is evident that the reconstructed Peso is unable to preserve the reference Peso's morphology.

The examples in this section show that at high SIRs, the quality of the reconstruction is visually very good, which is supported by a low PRD distortion measure. On the other hand, the reconstruction quality of Peso signals with low SIRs can be variable; some reconstructions appear to do well in suppressing CGO and preserving the Peso morphology while other reconstructions fail to do either. Nevertheless, it can be seen from Figure 4.16 that there is an increase in PRD values as the SIR goes up, which suggests that there is a relationship between the levels of CGO interference and the quality of the reconstruction.

In addition to the potential relationship between SIR and PRD, the examples in this section also show that the CGO interference can affect the amplitude and the swing of the actual Peso signal. The signals shown in Figure 4.16 specifically illustrate the aforementioned observation; 4.16(B) shows that the peak negative pressure can be significantly modified by the CGO while an 
amplitude change can be observed in 4.16(A) albeit to a lesser degree. The modification of the Peso swing by CGO was introduced in Section 4.1 as one of the key challenges when developing signal enhancement tools for Peso signals. Based on these results, clinicians must consider that the morphology of the $\Delta \mathrm{P}$ swing has been modified. These considerations should be incorporated into their visual assessments when tasked with face validation of reconstructed Peso signals from real humans.

\section{Relationship between SIR and PRD}

To determine the relationship between SIR and PRD for the FrankEinstein's "noisy" Peso signals, a scatterplot of the PRD with respect to its associated SIR was created and is shown in Figure 4.18. Using linear regression, a first-order polynomial was fit to the data. The regression line and the model coefficients are shown in red in Figure 4.18. Based on visual inspection of the data, it is evident that there is a negative relation between the independent variable, SIR, and the dependent variable, PRD. The negative relation is further confirmed by the negative slope of the linear regression model. The data suggests that FrankEinstein Peso signals with low SIR have significantly more error after reconstruction compared to Peso signals with high SIR.

In order to put the abovementioned observation into context, it is first important to understand the meaning behind SIR. A high SIR signifies that the signal's power is higher than the power of the interference. As a result, it is easier to detect the true signal. As the SIR level decreases, the power of the noise increases. Thus, the ability to detect the true signal becomes more difficult. In the research presented in this thesis, the aim of the proposed enhancement scheme is to preserve the Peso information by selecting the relevant IMFs while removing the information from the CGO interference. Keeping in line with the definition of SIR and its implications on signal detection, FrankEinstein signals with high SIR result in poor reconstructions because the 
ability to detect the actual Peso in the IMFs is hindered by the higher power levels of the CGO interference. Alternatively, the proposed enhancement scheme can detect the true Peso signal with ease at higher SIR levels, which lead to better CGO suppression and enhanced Peso reconstruction, thereby resulting in lower PRD values.

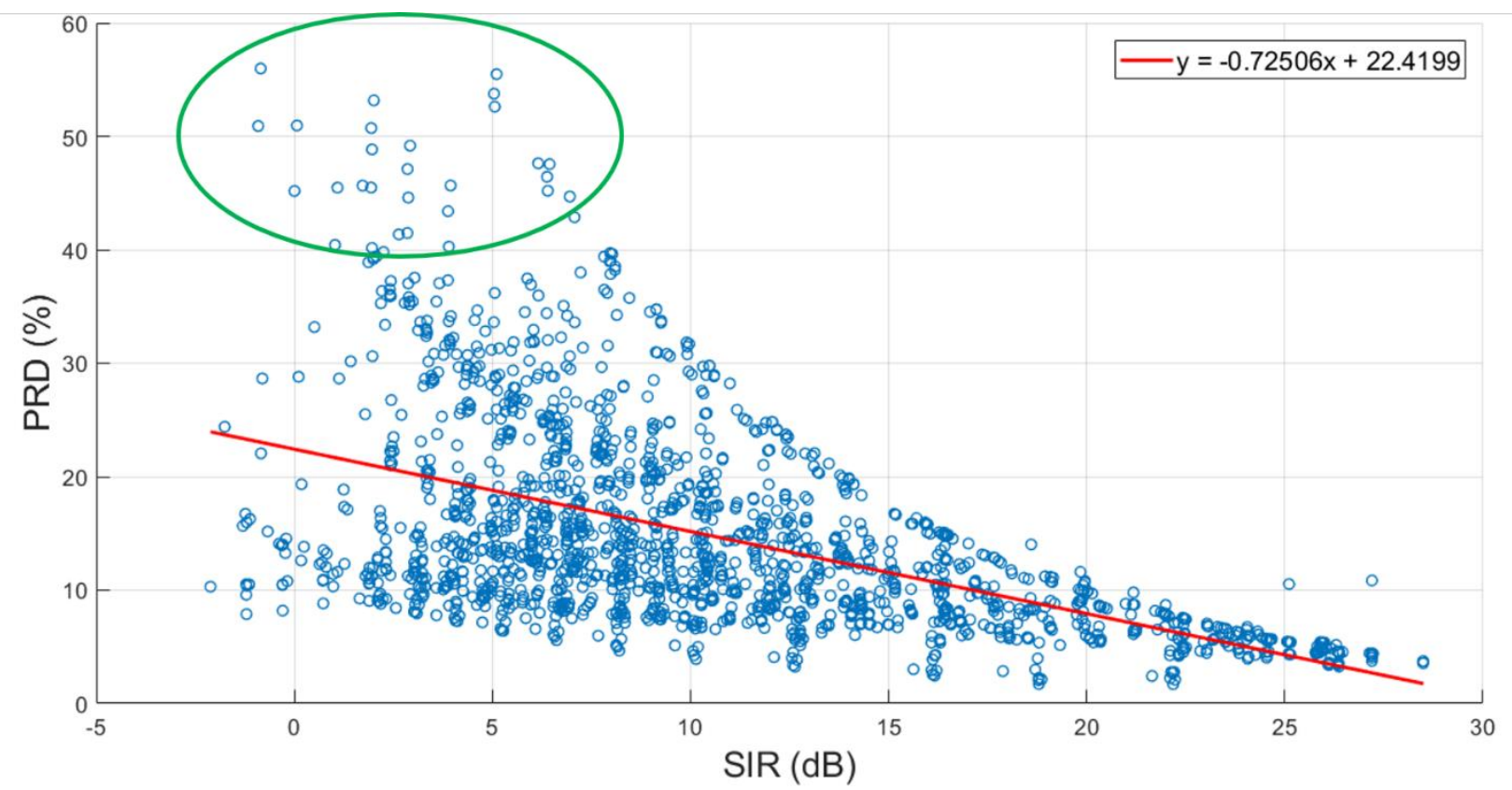

Figure 4.18. A scatterplot of PRD (\%) with respect to SIR (dB) for all 1800 "noisy" FrankEinstein Peso signals. A first-order polynomial model was fit to the data and is plotted in red. A region containing a low density of datapoints above PRD $=\sim 40 \%$ is outlined in a green circle. The datapoints represent significant outliers, most of which are associated with one specific CGO.

\section{Low-Density Region in PRD vs. SIR}

Another notable observation of the datapoints shown in Figure 4.18 is the low-density region above $\mathrm{PRD}=\sim 40 \%$, which is highlighted by a green circle. The datapoints within this region are considered outliers in the data. Upon further inspection of the datapoints in the abovementioned region, it was found that the majority of these points were associated with one 
specific CGO. It was found that the heart rate for the specific CGO was estimated to be around 54 bpm, which also happens to be the lower bound for heart rate in the 20 CGOs that were extracted.

Two possible factors may help to explain the poor reconstruction performance for these Peso signals. First, the SIR associated with these datapoints are quite low (generally less than 5 $\mathrm{dB})$. As previously mentioned, a low SIR means that significantly more CGO interference is present in the signal, which makes detecting the true Peso signal more difficult. The second factor is related to the estimated heart rate of the CGO itself. Because the CGO is relatively low, there is a possibility that the frequency spectrums of CGO and Peso overlap. In situations such as this one, it is impossible to truly separate the processes, because their frequency components are embedded within the same IMFs.

\section{Operating Limits of the Proposed Enhancement Scheme}

Another approach to quantifying the performance of the proposed enhancement scheme is by categorizing the PRD values according to which of the 10 scale factors was used in constructing the FrankEinstein Peso. By separating the PRD values according to the scale factor of the interference amplitude, it is possible to not only gain insights into how the technique performs at various interference levels, but also helps in determining the upper operating limits of the technique. To visualize the PRD measures with respect to their normalized interference amplitude, a box plot generated and shown in Figure 4.19. Each category (i.e. the 10 scale factors) contains 180 PRD values, which were derived from suppressing the CGO from 180 different FrankEinstein "noisy" Peso signals. Table 10 summarizes descriptive statistic measures of the PRD for each of the scale factors. Measures of central tendency include the mean and median, while the measures of variability are standard deviation, minimum, maximum, range, and skewness. 
From a holistic perspective, the mean and median of the PRD distributions for each of the scale factors shown in Figure 4.19 increases as the interference amplitude increases. In other words, as the interference scale factor increases, the quality of the Peso reconstruction after CGO suppression becomes increasingly distorted. This observation is consistent with the relationship shown in Figure 4.18 in which the PRD of the reconstructed Peso increases as the SIR decreases due to the inclusion of more interference power in the signal.

Looking more closely at the individual categories, the PRD distribution using an amplitude scaling factor of $0.1 \mathrm{cmH}_{2} \mathrm{O}$ is characterized by a mean of $5.4 \%$, a standard deviation of $1.7 \%$, and a range of $9.5 \%$. The minimum and maximum PRD are $1.7 \%$ and $11.2 \%$, respectively. Additionally, the boxplot indicates that the distribution lies completely below a PRD of $10 \%$ with the exception of four outliers. In terms of performance and reliability, the proposed enhancement scheme performs quite well due to a low standard deviation. A low standard deviation indicates that there is low variability of the reconstruction quality; the amount of information lost during CGO suppression and Peso reconstruction is consistently within a small range below $10 \%$. However, the performance of the reconstruction appears to degrade as the scaling factor increases; at an interference level of $0.2 \mathrm{cmH}_{2} \mathrm{O}$, the reconstruction performance mean is $8.7 \% \pm 3.2 \%$; when the interference level is set to $0.5 \mathrm{cmH}_{2} \mathrm{O}$, the mean PRD is $14.6 \% \pm 6.9 \%$; and at an interference level of $1 \mathrm{cmH}_{2} \mathrm{O}$, the performance is characterized by a mean PRD of $21.6 \% \pm 10.5 \%$.

To put the PRD values mentioned above and their relation to the performance of the proposed enhancement scheme into context, it is best to observe the statistical trends of the PRD at different interference levels. The PRD distributions for all categories are positively skewed, which indicates that the majority of the reconstructions tend to be within the lower range of the PRD distortion measure. However, as mentioned previously, the measures of central tendency 
increase as the interference scale factor increases, which manifests as more distortions in the Peso reconstructions as the amplitude of CGO gets larger. In addition to the increases observed in the measures of central tendency, the measures of variability also go up as the CGO gets larger. As an example of large variability in the PRD distribution, the boxplot for normalized interference amplitude of $1 \mathrm{cmH}_{2} \mathrm{O}$ shown in Figure 4.19 is used. The variability of the PRD distribution, which is numerically represented by a standard deviation of $10.5 \%$, a range of $48.1 \%$, and visually represented by a large interquartile range, is indicative of inconsistent reconstruction performance as shown by the wide range of PRD values. This is a direct contrast to the variability of the PRD distribution for a normalized interference amplitude of $0.1 \mathrm{cmH}_{2} \mathrm{O}$ in which the spread of the PRD values is close to the mean. The trends observed in the statistical measures can be attributed once again to the relationship described in the previous section; an increase in the interference amplitude makes it more difficult to detect the true Peso signal, which results in more distortions in the reconstruction. 


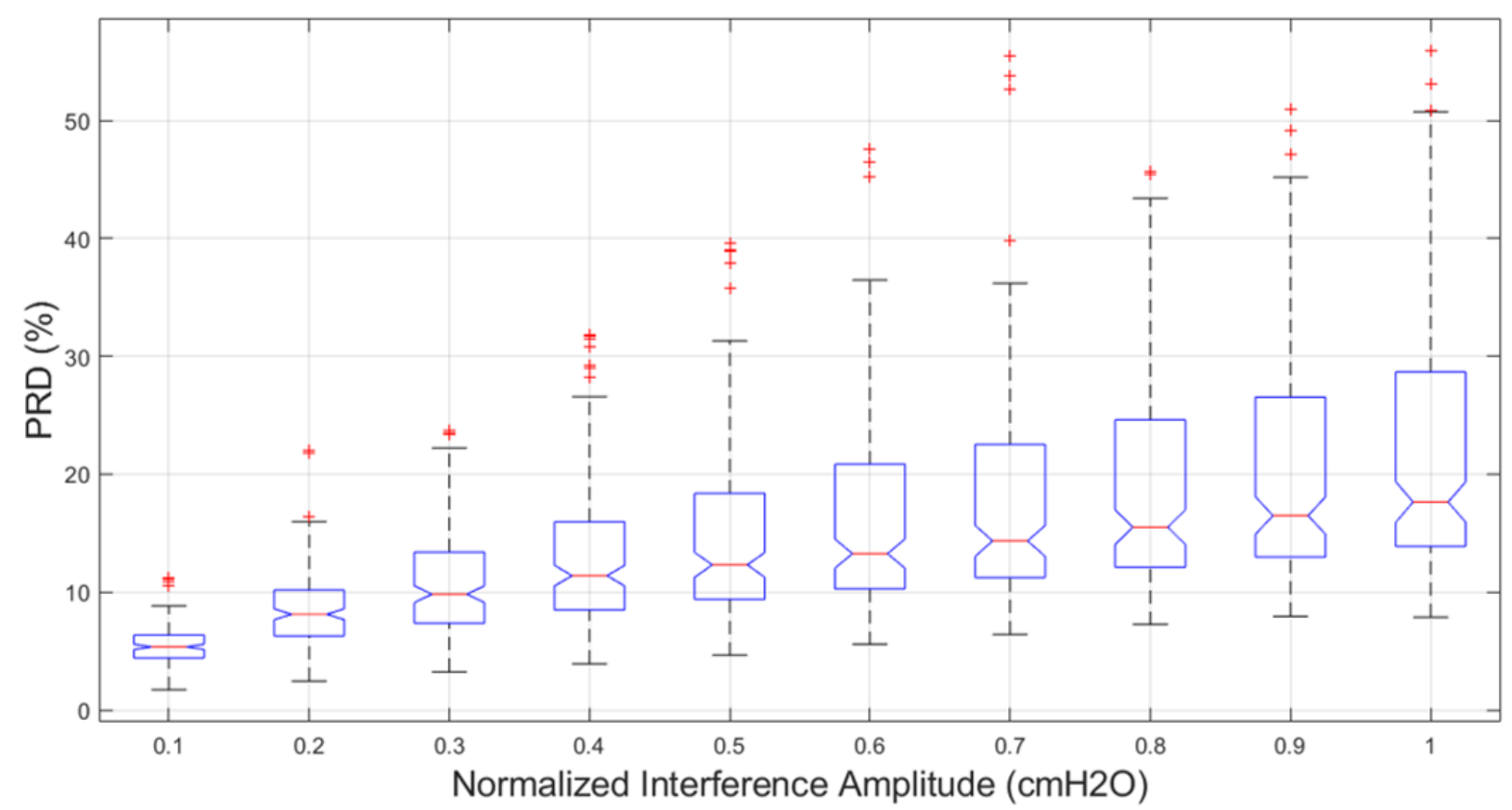

Figure 4.19. A boxplot showcasing the distribution of PRD (\%) for each of the 10 CGO scale factors.

TABLE 10: DESCRIPTIVE STATISTIC MEASURES OF PRD FOR EACH OF THE NORMALIZED CGO AMPLITUDE SCALE FACTORS.

\begin{tabular}{|c|c|c|c|c|c|c|c|}
\hline $\begin{array}{c}\text { Normalized } \\
\text { CGO } \\
\text { Amplitude } \\
(\mathbf{c m H 2})\end{array}$ & $\begin{array}{c}\text { Mean } \\
\text { PRD } \\
(\boldsymbol{\%})\end{array}$ & $\begin{array}{c}\text { Median } \\
\text { PRD } \\
(\boldsymbol{\%})\end{array}$ & $\begin{array}{c}\text { Standard } \\
\text { Deviation } \\
\text { of PRD } \\
(\boldsymbol{\%})\end{array}$ & $\begin{array}{c}\text { Min } \\
\text { PRD } \\
(\boldsymbol{\%})\end{array}$ & $\begin{array}{c}\text { Max } \\
\text { PRD } \\
(\boldsymbol{\%})\end{array}$ & $\begin{array}{c}\text { Range } \\
(\boldsymbol{\%})\end{array}$ & Skewness \\
\hline 0.1 & 5.398 & 5.351 & 1.705 & 1.706 & 11.164 & 9.458 & 0.632 \\
\hline 0.2 & 8.653 & 8.123 & 3.240 & 2.456 & 22.068 & 19.612 & 1.174 \\
\hline 0.3 & 10.851 & 9.820 & 4.392 & 3.237 & 23.731 & 20.493 & 0.973 \\
\hline 0.4 & 12.922 & 11.395 & 5.960 & 3.924 & 31.832 & 27.908 & 1.236 \\
\hline 0.5 & 14.553 & 12.313 & 6.992 & 4.659 & 39.629 & 34.970 & 1.375 \\
\hline 0.6 & 16.237 & 13.263 & 7.974 & 5.580 & 47.552 & 41.972 & 1.352 \\
\hline 0.7 & 17.697 & 14.344 & 8.868 & 6.415 & 55.478 & 49.063 & 1.520 \\
\hline 0.8 & 18.993 & 15.508 & 8.951 & 7.261 & 45.665 & 38.404 & 0.970 \\
\hline 0.9 & 20.204 & 16.490 & 9.646 & 7.951 & 50.952 & 43.001 & 1.040 \\
\hline 1.0 & 21.634 & 17.640 & 10.465 & 7.880 & 55.977 & 48.097 & 1.068 \\
\hline
\end{tabular}


To determine the upper operating limit of the proposed enhancement scheme, recall that in Chapter 3 the collaborating clinicians provided a tolerance of 10\% loss in Peso information. For an interference scaling factor of $1 \mathrm{cmH}_{2} \mathrm{O}$, the measures of central tendency and variability of the PRD distributions was consistently below 10\%. These results suggest that the proposed enhancement scheme can be used when the CGO amplitude is $\sim 10 \%$ of the amplitude of the Peso swing, $\Delta \mathrm{P}$. For an interference scaling factor of $0.2 \mathrm{cmH}_{2} \mathrm{O}$, the mean PRD was found to be $8.7 \%$ $\pm 3.2 \%$. Additionally, the third quartile, as seen from Figure 4.19, was found to be a PRD of $10.2 \%$, which means that $75 \%$ of the 180 (i.e. 135) "noisy" Peso signals with a CGO amplitude of $0.2 \mathrm{cmH}_{2} \mathrm{O}$ had a PRD less than or equal to $10.2 \%$. The measures of central tendency for the interference scaling factor of $0.3 \mathrm{cmH}_{2} \mathrm{O}$ appear at the borderline of the clinical threshold with a mean of $10.9 \% \pm 4.4 \%$. The median PRD is $9.8 \%$, which indicates that $50 \%$ of the reconstruction PRDs were within the permitted threshold. These statistical measures suggest that the proposed enhancement scheme may be used to suppress CGO interference that is $\sim 20 \%$ the amplitude of $\Delta \mathrm{P}$ reliably and until $\sim 30 \%$ the amplitude of $\Delta \mathrm{P}$, but with the caveat that there is a possibility of surpassing the $10 \%$ information loss threshold. Based on the $10 \%$ threshold for Peso information loss imposed by clinicians, the remaining interference amplitude categories cannot suppress CGO without losing significant Peso information.

\subsection{Chapter Summary}

This Chapter focused on two main ideas: the creation of FrankEinstein's "noisy" Peso signals, and applying the proposed enhancement scheme to the FrankEinstein data. Generating our own synthetic data was in response to the fact that no objective and quantifiable ways of benchmarking the CGO suppression performance was available for real patients. Although face validation from clinical experts was used to gain insights into the performance of the proposed 
enhancement scheme, their opinions were based on subjective visual assessments of the reconstructed Peso signals. While face validation may be considered the "gold standard," personal biases such as the clinicians' tolerance for filtering as well as the lack of a reference/true Peso signal, contribute to compromising the fidelity of performance validation.

Sections 4.2 and 4.3 were devoted to describing the characteristics and the methods used to obtain the two components of the FrankEinstein Peso. The Peso used as the base/reference for FrankEinstein's Peso signal was shown in Section 4.2. The ASL 5000 breathing simulator was used to simulate nine spontaneously breathing patients with the assumptions that the lungs are modelled as a single compartment, and that the patient has a sinusoidal breathing pattern. The morphology of the raw ASL 5000 Peso signals were found to have unnaturally sharp/sudden transitions between the inspiration and expiration phases, which are not characteristic of human breathing. Thus, a 70-pt moving average filter was applied to the raw ASL 5000 signals to mimic a real human Peso morphology. The second component of FrankEinstein's Peso, which was described in Section 4.3, was the CGO. The CGOs were extracted using EEMD from 20 randomly selected Peso signals from a database of 75 Peso signals captured in the ICU. In both sections, the final waveforms were normalized to have amplitudes between -1 to $1 \mathrm{cmH}_{2} \mathrm{O}$.

In Section 4.4, the base Peso signals and the CGOs were superimposed onto each other to generate the FrankEinstein "noisy" Peso signal database. Each of the 20 CGO signals were scaled 10 different times with scaling factors ranging between $0.1 \mathrm{cmH}_{2} \mathrm{O}$ to $1 \mathrm{cmH}_{2} \mathrm{O}$ in $0.1 \mathrm{cmH}_{2} \mathrm{O}$ increments. Thus, it was possible to superimpose 200 different CGOs on the nine base Peso signals, which resulted in a database size of 1800 "noisy" Peso signals. For each of the 10 scaling factors, there were 180 unique Peso signals with different CGOs. By varying the amplitude levels of the CGO and keeping the amplitude of the base Peso constant, it was then be possible to test the 
operating limits of the proposed technique. Through this methodology, we were able to augment the size of the datasets used in Chapter $\mathbf{3}$ to overcome the "small dataset" problem, as well as test the robustness of the proposed enhancement scheme to different levels of interference.

In Section 4.5, the proposed enhancement scheme was applied to each of the 1800 FrankEinstein Peso signals. Because the reference signal (i.e. the "true" Peso) was readily available, it was possible to validate the CGO suppression performance not only through visual means, but through quantifiable distortion measures, such as PRD, as well. An automated IMF selection method was introduced into the proposed scheme due to the reliance on clinicians to manually select the IMFs, which is not feasible for large datasets such as FrankEinstein's "noisy" Peso signals. The results from applying the modified proposed enhancement scheme show that the level of interference can modify the morphology of the Peso, specifically at the $\Delta \mathrm{P}$ swing. Moreover, varying the levels of CGO amplitude also affects the reconstruction performance; in some cases, the reconstruction was able to suppress the CGO quite well while preserving the Peso morphology. However, there were also reconstructions that resulted in very high PRD values, indicating that extreme distortions were present. By plotting the reconstruction PRD for each of the FrankEinstein signals with respect to their associated SIR, it became apparent that lower SIRs generally led to higher PRD values. This relationship observed between SIR and PRD can be explained by the fact that an increase in the interference level makes it difficult for the proposed enhancement scheme to detect the "true" Peso signal.

Despite a general trend for the relationship between PRD and SIR being established, the question as to how much interference can the proposed enhancement scheme handle arises. To test the operating limits of the proposed enhancement scheme, a boxplot of the reconstruction PRDs categorized into their respective normalized interference amplitude was generated. Additionally, 
measures of central tendency and variability were calculated for each category. Two conditions were considered in determining if the proposed enhancement scheme can be used: i) the majority of the PRD distribution fell at or below $10 \%$ as per the clinician-defined tolerance for Peso loss, and ii) the reconstruction performance was consistent, which are indicated by the measures of variability as well as the interquartile range in the boxplot. Based on the results obtained in this section, it was found that the proposed enhancement scheme could reliably be used up until the CGO amplitude is $20 \%$ of the $\Delta \mathrm{P}$ amplitude. However, it has also been suggested that the enhancement scheme can still be used until the CGO amplitude is $\sim 30 \%$ of $\Delta \mathrm{P}$, but under the condition that clinicians are able to tolerate slightly more than $10 \%$ Peso information loss.

In this Chapter, we were able to address some of the challenges mentioned in Section 4.1. The creation of the FrankEinstein Peso dataset provided us with the ability to overcome the "small dataset" problem. Furthermore, we were also able to verify that the CGO can in fact change the shape of the Peso signal. We were also able to quantify the proposed enhancement scheme's performance to establish a relationship between PRD and SIR. These quantifiable measures were also instrumental in determining which levels of interference the enhancement scheme can reliably be used. Through these results, recommendations can be provided to clinicians if/when the proposed enhancement scheme is translated from development to clinical use. It is important to note however that the gold standard for validation still lies with the clinician; it is to their discretion as to whether the performance of the proposed enhancement scheme can provide clinically viable Peso signals for different levels of CGO interference. Additionally, it is essential to recognize the limitations of the Peso signals generated by the ASL 5000. Despite being a state-of-the-art breathing simulator, it is still unable to mimic the complex interactions of the respiratory system in mechanically ventilated patients. 


\section{Chapter 5}

\section{CGO Suppression in Real ICU Patients}

\subsection{Motivation}

In Chapters 3 and 4, the proposed Peso enhancement scheme was refined by establishing the appropriate decomposition algorithm for CGO suppression and defining an automated IMF selection for reconstruction. While the FrankEinstein Peso signals introduced in Chapter 4 served as a means of testing the performance of our technique, the models used to generate the "true" Peso signal were limited in their ability to reproduce the complex physiological interactions of the respiratory system in mechanically ventilated patients. The aims of this Chapter are to determine if the proposed technique is robust enough to suppress CGO from ICU patients, and to see if the resulting Peso signal is clinically viable from the perspective of critical care specialists.

\subsection{Application of the Proposed Enhancement Scheme on Real ICU Patients}

\subsubsection{Dataset}

The dataset used in this section was described in Chapter 4 under Section 4.3.1. Recall that this dataset contains 25 different patients under three different breathing conditions: i) passive breathing, ii) spontaneous breathing with PSV, and iii) breathing with a t-piece, resulting in 75 signals. This dataset contains five types of data: i) Airway Pressure $\left[\mathrm{cmH}_{2} \mathrm{O}\right]$, ii) Esophageal Pressure $\left[\mathrm{cmH}_{2} \mathrm{O}\right]$, iii) Gastric Pressure $\left[\mathrm{cmH}_{2} \mathrm{O}\right]$, iv) Flow [L/s], and v) Transpulmonary Pressure [ $\left.\mathrm{cmH}_{2} \mathrm{O}\right]$. The signals were sampled at $200 \mathrm{~Hz}$, and the duration of the signals range between 50.92 seconds to 644.86 seconds. The collection of this data was approved by St. Michael's Hospital's 
Research Ethics Board under REB\# 15-369. A summary of the data used in this section is shown in Table 11.

TABLE 11: SUMMARY OF THE DATASET USED FOR TESTING THE FINAL PROPOSED ENHANCEMENT SCHEME.

\begin{tabular}{|c|c|c|c|c|c|c|c|}
\hline $\begin{array}{c}\text { Dataset } \\
\#\end{array}$ & $\begin{array}{c}\text { REB } \\
\#\end{array}$ & $\begin{array}{l}\text { No. of } \\
\text { Patients }\end{array}$ & $\begin{array}{l}\text { Condition of } \\
\text { Patients }\end{array}$ & Types of Data & $\begin{array}{l}\text { Sampling } \\
\text { Frequency } \\
\text { (Hz) }\end{array}$ & $\begin{array}{l}\text { Duration } \\
\text { of } \\
\text { Signals } \\
\text { (sec) }\end{array}$ & $\begin{array}{c}\text { Total } \\
\text { No. of } \\
\text { Signals }\end{array}$ \\
\hline 3 & $\begin{array}{l}15- \\
369\end{array}$ & 25 & $\begin{array}{ll}\text { 1. } & \text { Passive } \\
\text { 2. Spontaneous } \\
\text { with PSV } \\
\text { 3. Breathing } \\
\text { with t-piece }\end{array}$ & $\begin{array}{l}\text { 1. Airway } \\
\text { Pressure } \\
\text { 2. Esophageal } \\
\text { Pressure } \\
\text { 3. Gastric Pressure } \\
\text { 4. Flow } \\
\text { 5. Transpulmonary } \\
\text { Pressure }\end{array}$ & 200 & $\begin{array}{l}50.92- \\
644.86\end{array}$ & 75 \\
\hline
\end{tabular}

\subsubsection{Experimental Setup}

\section{Method for Suppressing CGO in Peso}

The proposed enhancement scheme that was developed throughout this thesis will be applied to all 75 Peso signals described in Section 5.2.1. The EEMD algorithm will be applied, and 100 ensemble trials are used for decomposition. The amplitude of white noise added into each trial is shown in EQ5.1.

$$
n=0.2 \times \operatorname{std}(\text { Peso }) \quad(\text { EQ5. 1) }
$$

The automatic IMF selection approach described in Chapter $\mathbf{4}$ will be used. Recall that the IMF selection process involves finding the frequency bin associated with the largest magnitude in each of the IMF magnitude spectra. A threshold criterion of $0.75 \mathrm{~Hz}$ is defined; if the magnitude peak in the IMF spectra resides in a bin greater than $0.75 \mathrm{~Hz}$, the IMF is representative of CGO and other high-frequency processes present in the signal. 


\section{Validation of Results}

Face Validation from our clinical collaborators will be used to assess the clinical viability of the enhanced Peso signals. Clinicians will be making their decisions based on the quality of the enhanced Peso signal in terms of its morphology, slope of the Peso deflection $\left(\frac{d P e s o}{d t}\right)$, and the Peso $\operatorname{swing}(\Delta \mathrm{P})$.

\subsubsection{Results and Discussions}

\section{Representative Results}

In this section, 7 of the 75 Peso signals were selected to demonstrate the performance of the enhancement scheme. Figures 5.1 to 5.6 demonstrate representative results.

Figure 5.1 is a Peso signal obtained from a spontaneously breathing patient receiving PSV. The morphology/breath profile seen in this Figure represents a typical pattern observed in spontaneously breathing patients. This patient has a heart rate of $84 \mathrm{bpm}$ and the CGO amplitude is $\sim 2 \mathrm{cmH}_{2} \mathrm{O}$. The reconstructed Peso shown in red indicates that the technique has been able to suppress a significant amount of CGO activity by reducing its amplitude to $\sim 0.5-0.75 \mathrm{cmH}_{2} \mathrm{O}$. Despite the persisting CGO activity, our collaborating clinicians are satisfied with the suppression

performance, because the Peso morphology, $\frac{d P e s o}{d t}$, and $\Delta \mathrm{P}$ are largely unaffected based on Face Validation. 


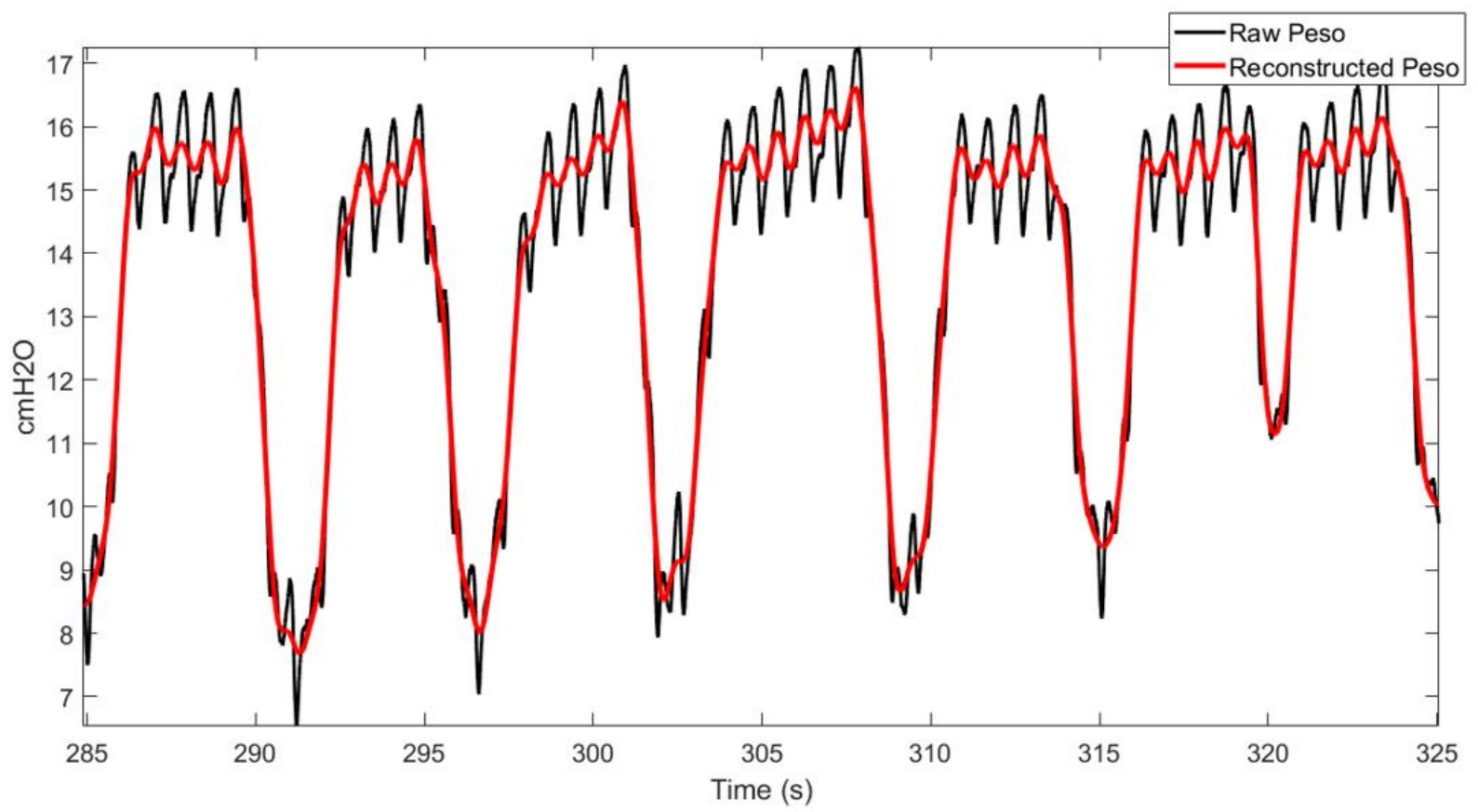

Figure 5.1. A Peso signal obtained from a spontaneously breathing patient receiving PSV. This Peso signal is representative of a typical breathing pattern and morphology.

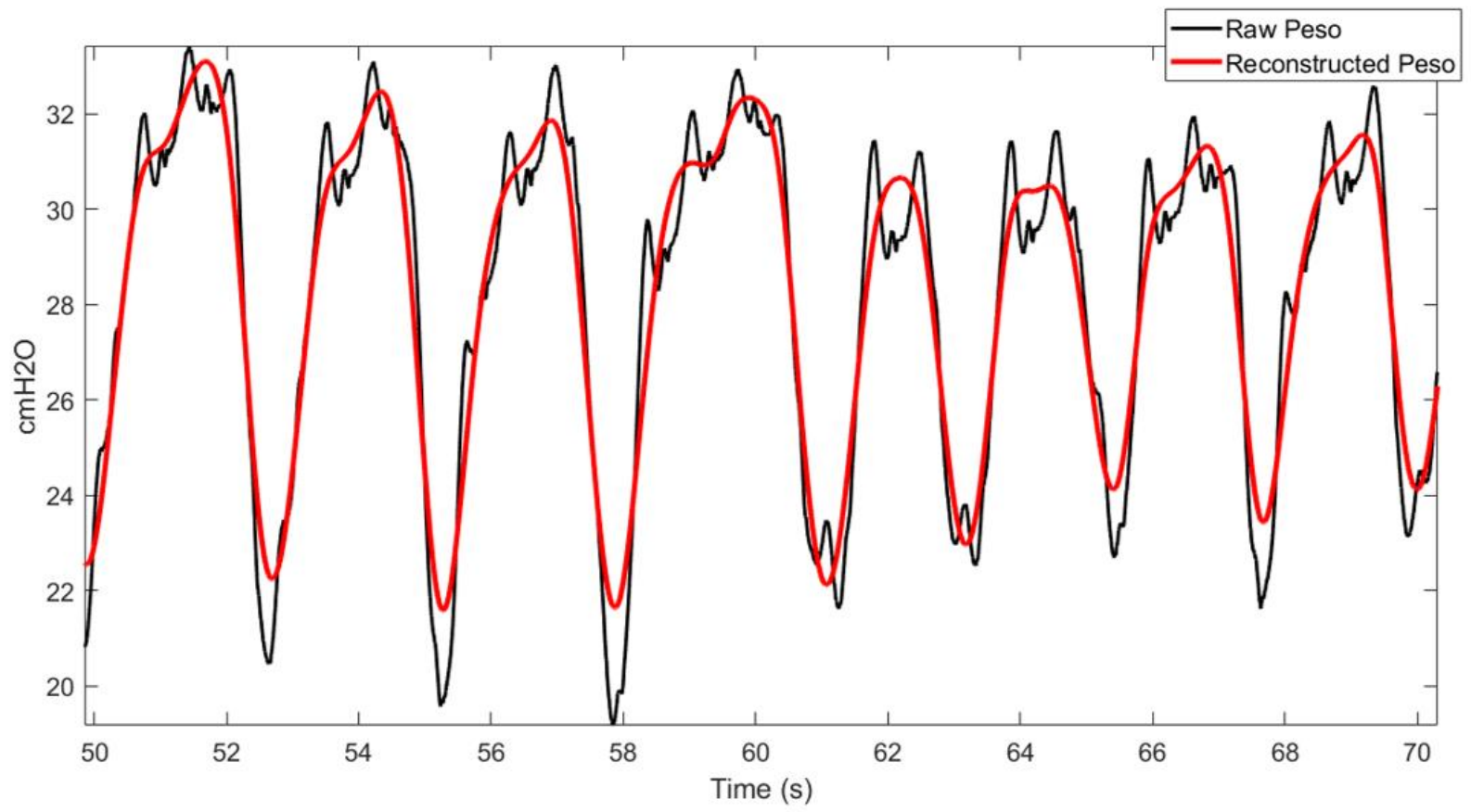

Figure 5.2. A Peso signal obtained from a spontaneously breathing patient receiving PSV. This Peso signal is representative of a similar breath profile/morphology shown in Figure 5.1, but with a different CGO morphology. 
Figure 5.2 is another Peso signal obtained from a spontaneously breathing patient receiving PSV. The morphology of this Peso signal is similar to the one shown in Figure 5.1, albeit this patient has a noticeably different CGO morphology. Furthermore, the amplitude of the Peso is not consistent; the first 10 seconds illustrate a consistently large $\Delta \mathrm{P}$, which may indicate that the patient is taking deep breaths, while the remaining 10 seconds show a consistently smaller $\Delta \mathrm{P}$. Unlike in Figure 5.1, the proposed technique has almost entirely suppressed the CGO. However, the collaborating clinicians have expressed concerns regarding the amplitude loss of $\Delta \mathrm{P}$, specifically at the inflection points between the inspiratory and expiratory phases. The loss of amplitude is most evidently observed in the first 10 seconds of the signal. The original Peso had a $\Delta \mathrm{P}$ of $\sim 12 \mathrm{cmH}_{2} \mathrm{O}$ and the reconstructed $\Delta \mathrm{P}$ was $\sim 10 \mathrm{cmH}_{2} \mathrm{O}$, which corresponds to a $16.7 \%$ loss in amplitude. Recall that the clinicians suggested a 10\% threshold on information loss. Despite surpassing the threshold, the reconstructed Peso can arguably be used for interpretation and analysis. Furthermore, as discussed in Chapters 3 and 4, the original $\Delta \mathrm{P}$ may have been influenced by the amplitude of the CGO interference, which has now been removed through the proposed technique.

Figure 5.3 is obtained from a spontaneously breathing patient receiving PSV and illustrates another commonly seen morphology/breath profile. The resulting Peso reconstruction has many similarities to the results seen in Figure 5.2; the CGO has almost entirely been suppressed and there is some information loss at the inflection points between the inspiratory and expiratory phases. Despite the information loss, the clinicians were satisfied with the suppression performance, because the reconstructed signal appears to represent the morphology of the Peso quite well. 


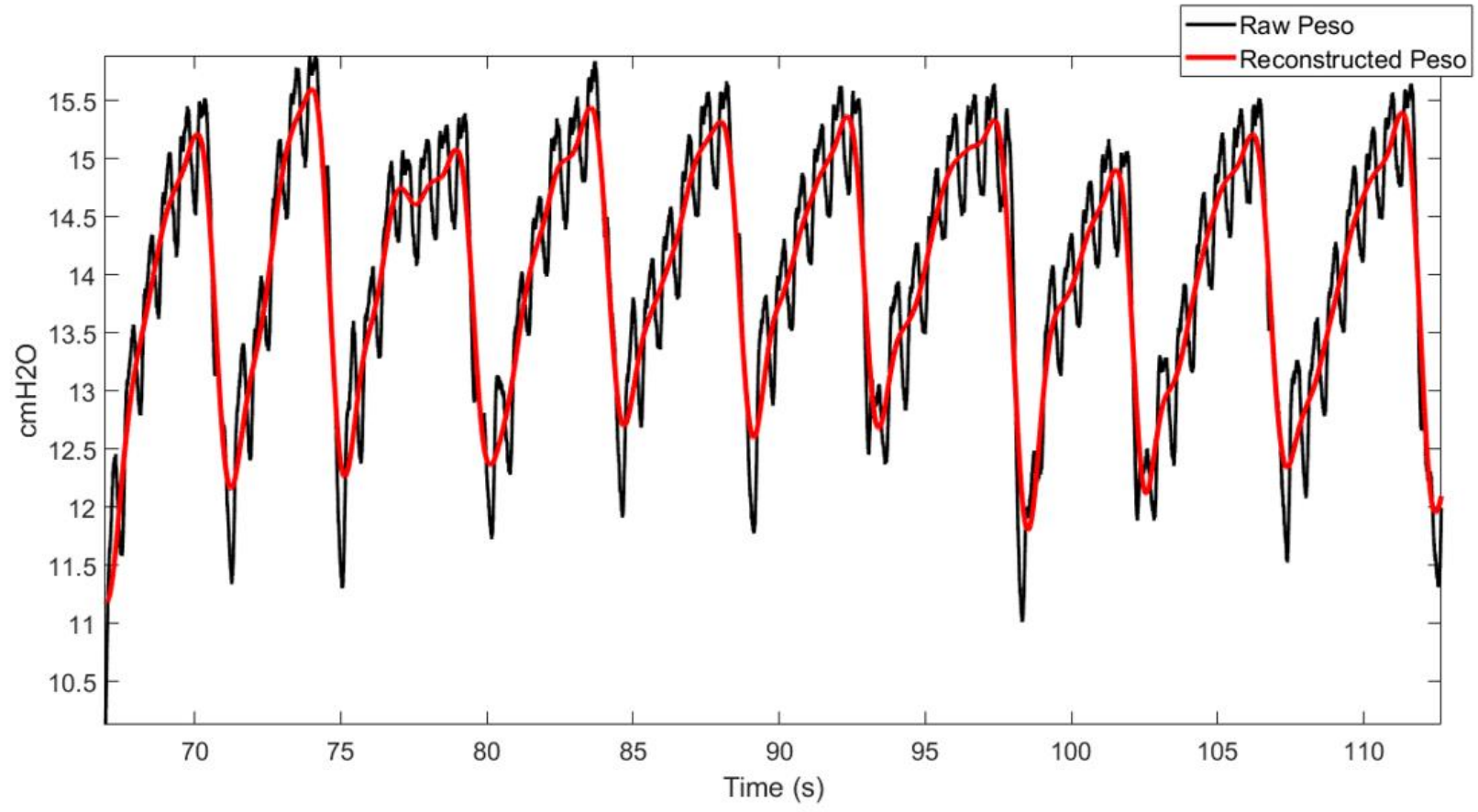

Figure 5.3. A Peso signal obtained from a spontaneously breathing patient receiving PSV. This Peso signal is representative of another commonly observed breath profile/morphology.

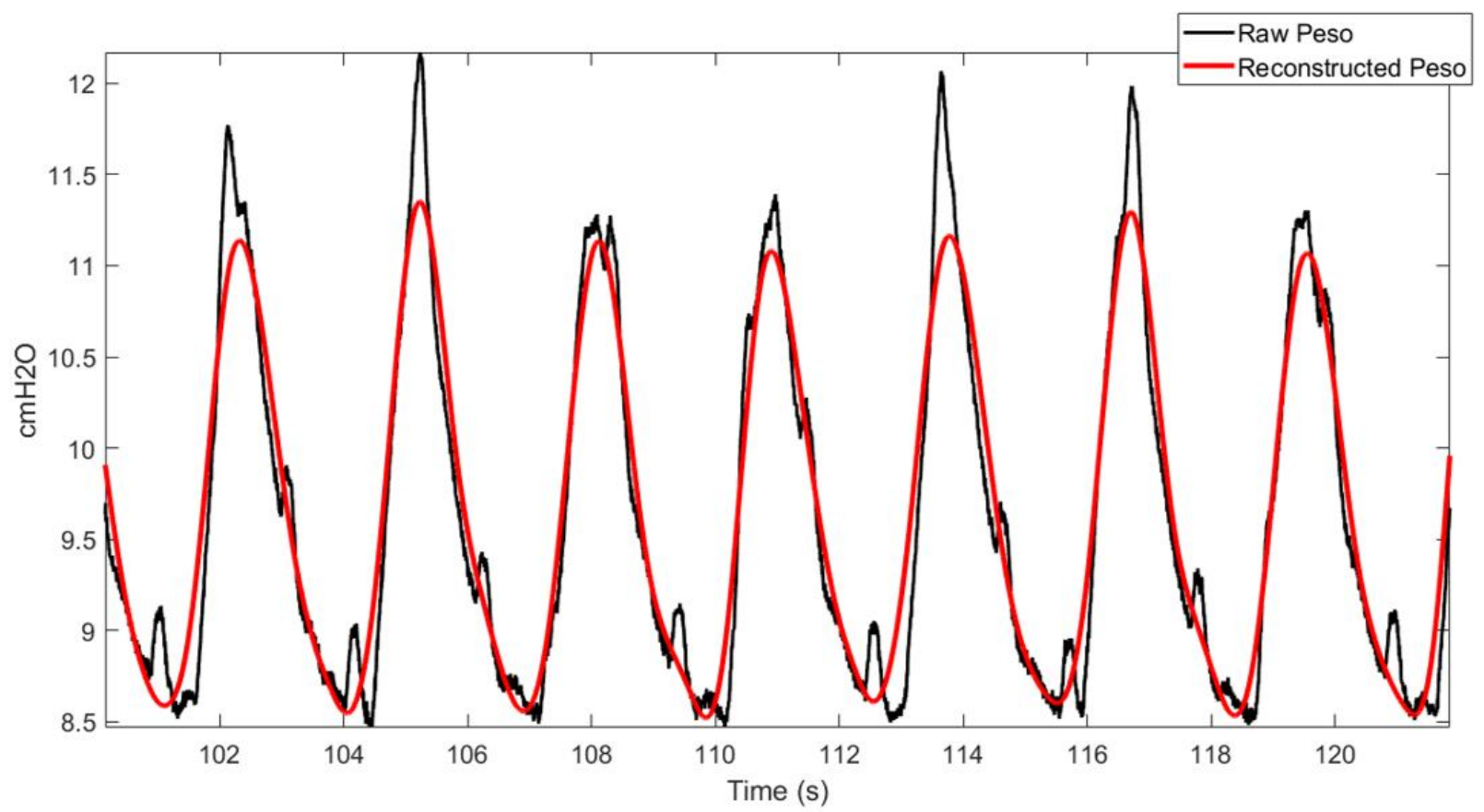

Figure 5.4. A Peso signal obtained from a passively breathing patient receiving mechanical ventilation support. 
Figure 5.4 is representative of a Peso signal obtained from a passively breathing patient receiving mechanical ventilation support. Generally, the reconstruction is agreed to be quite good and is able to suppress CGO. However, like the previous two Peso signals, there are some concerns regarding the loss of information at the inflection points (located at the top in this case due to the passive breathing condition).

Figure 5.5 is another Peso signal obtained from a passively breathing patient. This signal was selected to demonstrate the performance of the technique when an occlusion (i.e. a blockage) is present in the signal. The occlusion occurs at $t=41$ seconds to $t=46$ seconds, which means that the Peso should not deflect in any direction, and only the CGO will be observed in that timeframe. The reconstructed signal in Figure 5.5 shows no deflections or fluctuations present during the occlusion. These results therefore suggest that the proposed technique has been able to completely suppress the CGO, and lends confidence that the enhancement scheme is working as expected. Moreover, the morphology of the Peso deflections is deemed acceptable by the clinicians with the caveat that potential information has been lost at the inflection points.

Figure 5.6 is a Peso signal obtained from a spontaneously breathing patient using a t-piece. Just like in Figure 5.5, this signal was selected due to an occlusion being present in the signal. The occlusion occurs from $t=154$ seconds to $t=172$ seconds. The reconstructed signal shows that the CGO amplitude has been mostly been supressed, but there are small fluctuations still present. While the Peso in Figure 5.5 was expected to have no Peso deflections, that is not the case in spontaneously breathing patients. Because this patient's respiratory drive has not been suppressed, it is possible to see small deflections due to Peso activity that indicate breathing efforts by the patient. The consensus for reconstructed signal is that the CGO suppression was quite good, and the Peso morphology, for the most part, has been preserved. 


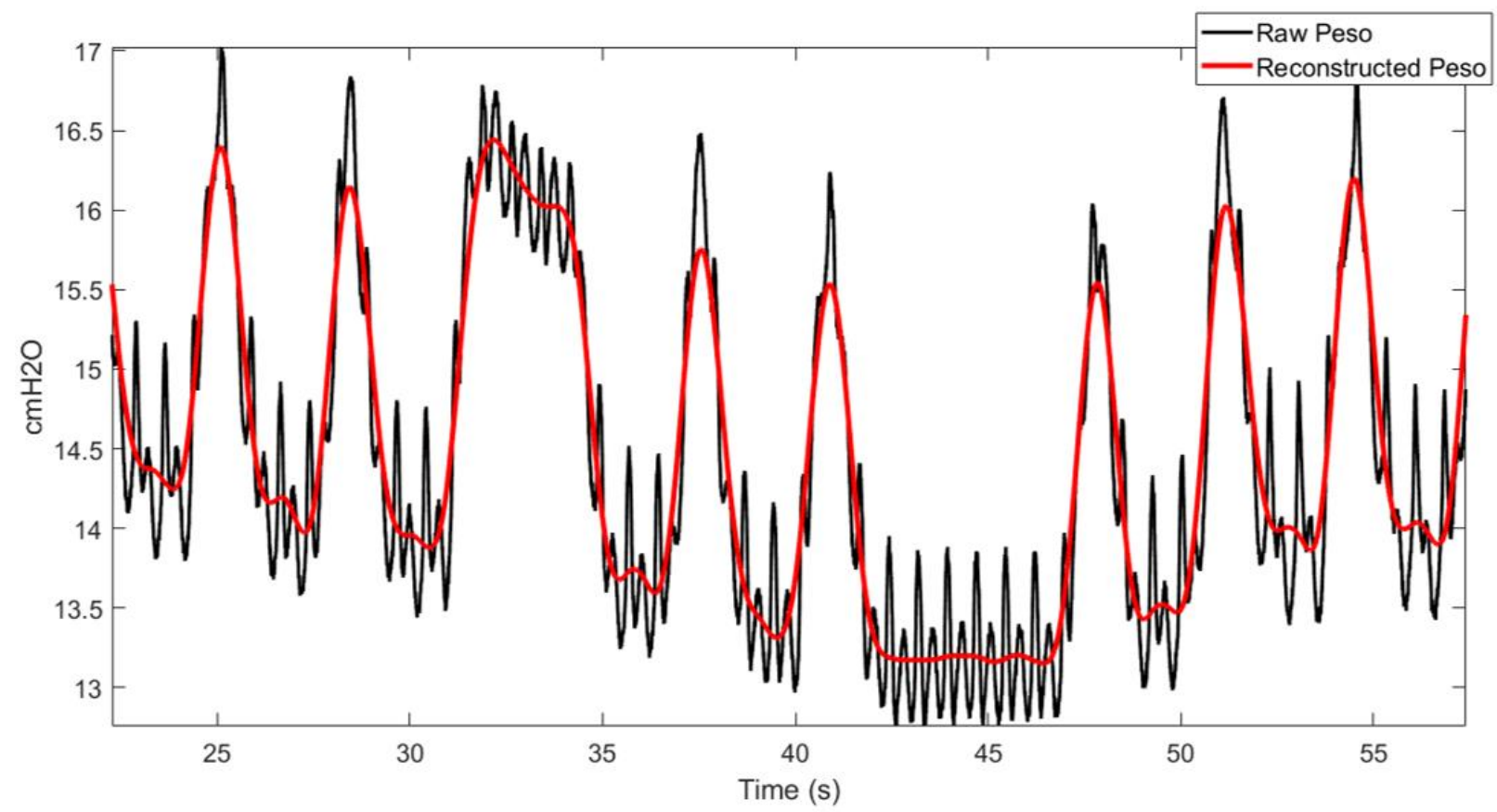

Figure 5.5. A Peso signal obtained from a passively breathing patient. An occlusion occurs at $t=$ 41 seconds to $t=46$ seconds.

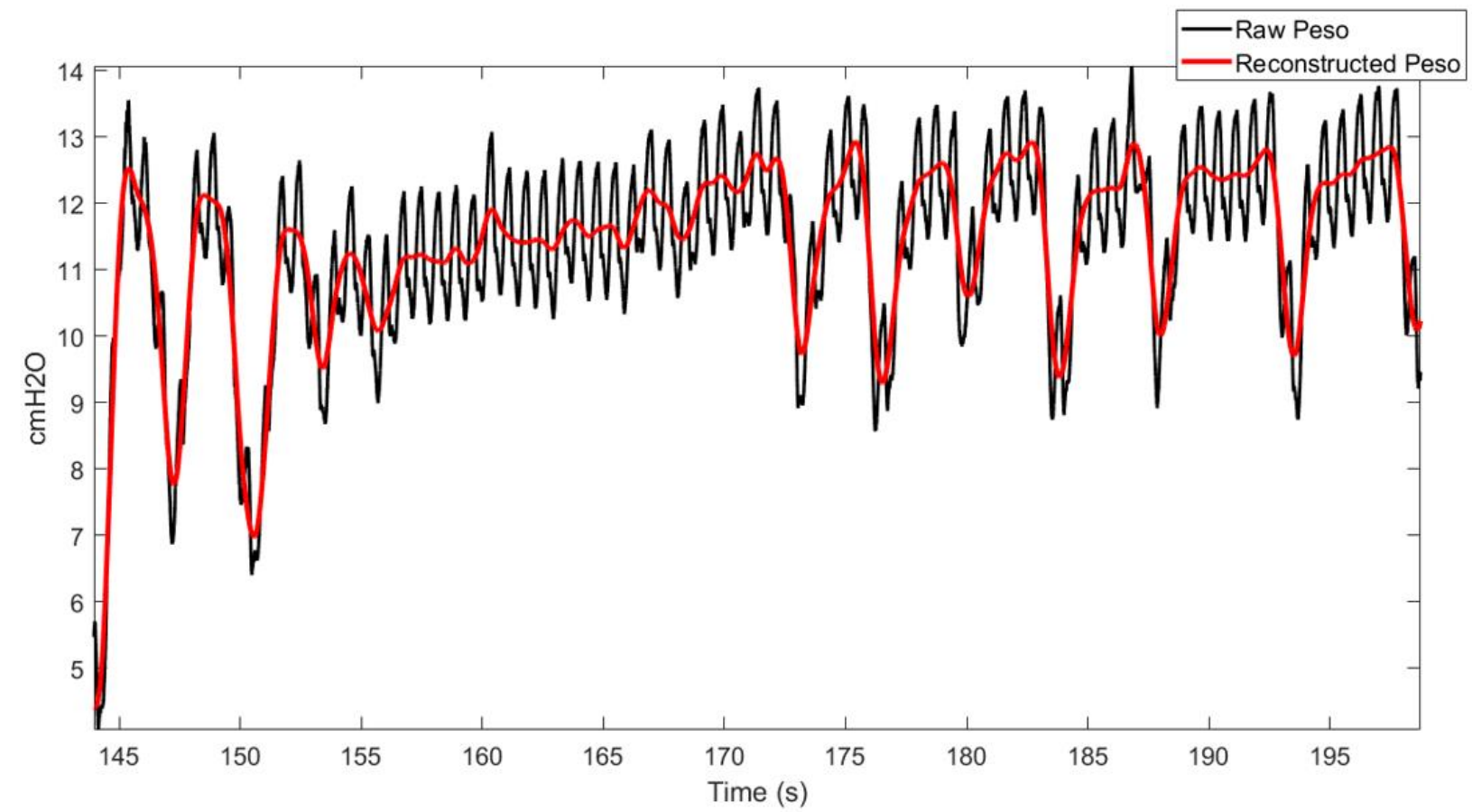

Figure 5.6. A Peso signal obtained from a spontaneously breathing patient using a t-piece. An occlusion occurs from $\mathrm{t}=154$ seconds to $\mathrm{t}=172$ seconds. 


\section{Limitations of the Technique}

\section{Effects of Esophageal Spasms}

Although CGOs are the main source of interference observed in the Peso signal, another prominent interference source are esophageal spasms that manifest as large transients in the signal as demonstrated in Figure 5.7. Because the balloon catheter is placed in the esophagus, any processes that cause variations in pressure within the esophagus are captured. The esophageal spasms are a result of physiological processes such as swallowing or peristalsis, which are rhythmic contractions of the esophagus. During interpretation of the Peso signal, clinicians will take note of when the esophageal spasm occurs and will not consider the subsequent Peso epochs for analysis. Analysis and interpretation the Peso signal will be stopped until the signal returns to a stable value.

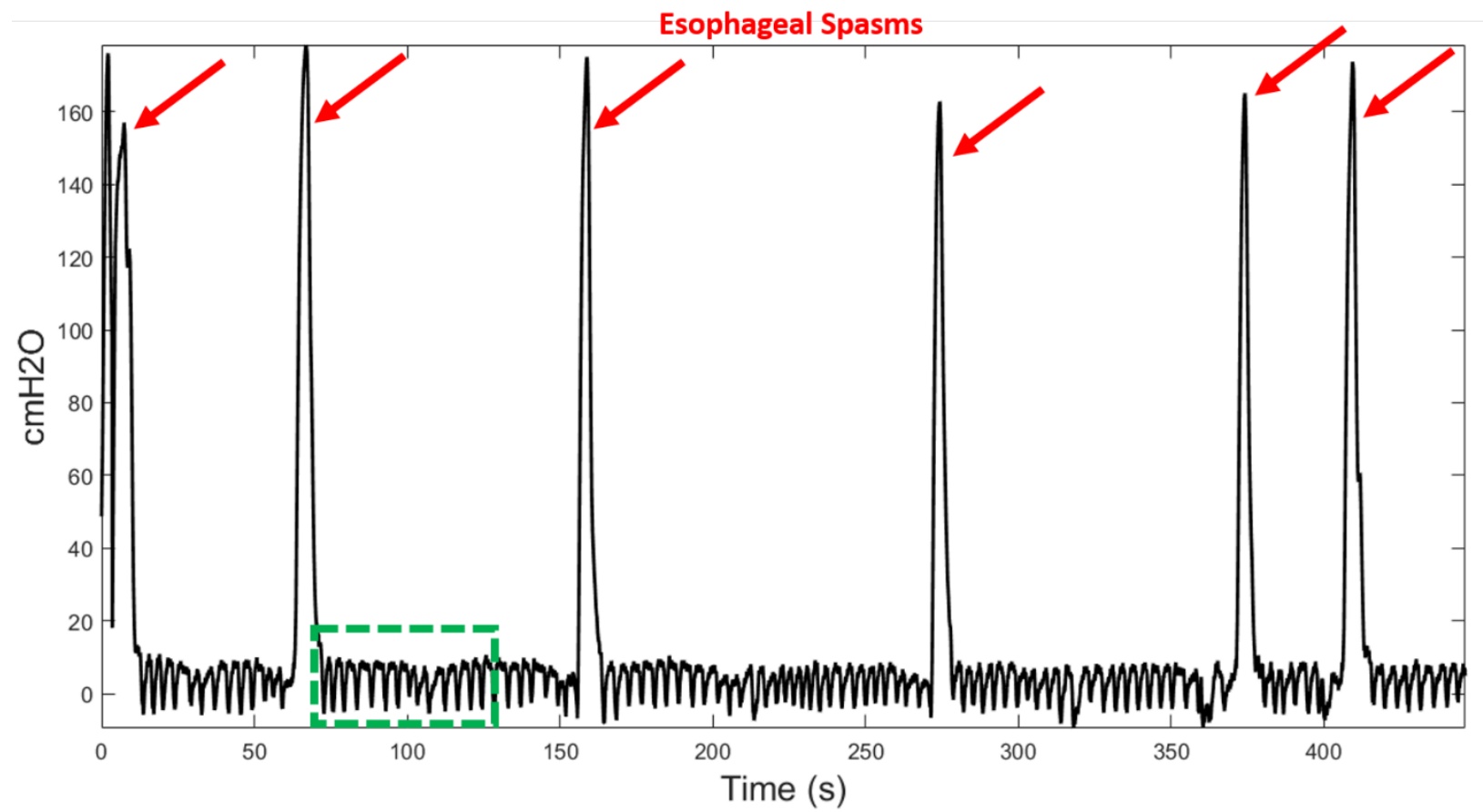

Figure 5.7. A Peso signal obtained from a spontaneously breathing patient receiving PSV. The large transients represent esophageal spasms caused by the patient swallowing their saliva. The segment of the signal highlighted in the green box is used in Figure 5.9 to illustrate the effects of the esophageal spasm on signal reconstruction using the proposed enhancement scheme. 
To see what effects the esophageal spasms have on the reconstruction of the Peso signal, the proposed enhancement scheme was applied under two different conditions:

1) The raw signal was decomposed without any modifications, and

2) The esophageal spasms were clipped before decomposition.

The Peso segment highlighted in the green box in Figure 5.7 is shown in Figure 5.8 for the purposes of making a direct comparison between the abovementioned conditions. In Figure 5.8 (A), which corresponds to Condition \#1, the reconstructed Peso (red) indicates that the proposed enhancement scheme has performed quite poorly; in fact, the quality of the resulting reconstruction may arguably be worse than the raw signal. In contrast, Figure 5.8 (B) shows a significantly better Peso reconstruction/CGO suppression (blue) under Condition \#2.

The poor reconstruction under Condition \#1 can be attributed to the inclusion of the esophageal spasm during decomposition, which affected the resulting IMFs. Because the esophageal spasm manifests as transients with significantly larger amplitude values than the Peso signal, the SIR is small compared to a large SIR under Condition \#2. As a result, the EEMD algorithm may be unable to separate the processes properly and preserve the Peso morphology. The issues introduced by the esophageal spasms is reminiscent of the FrankEinstein Peso signals with low SIR shown in Figure 4.17 of Section 4.5.3 in Chapter 4. Thus, the inclusion of esophageal spasms poses a challenge in preserving the Peso morphology using the proposed technique, which indicates that the spasms must first be removed. 

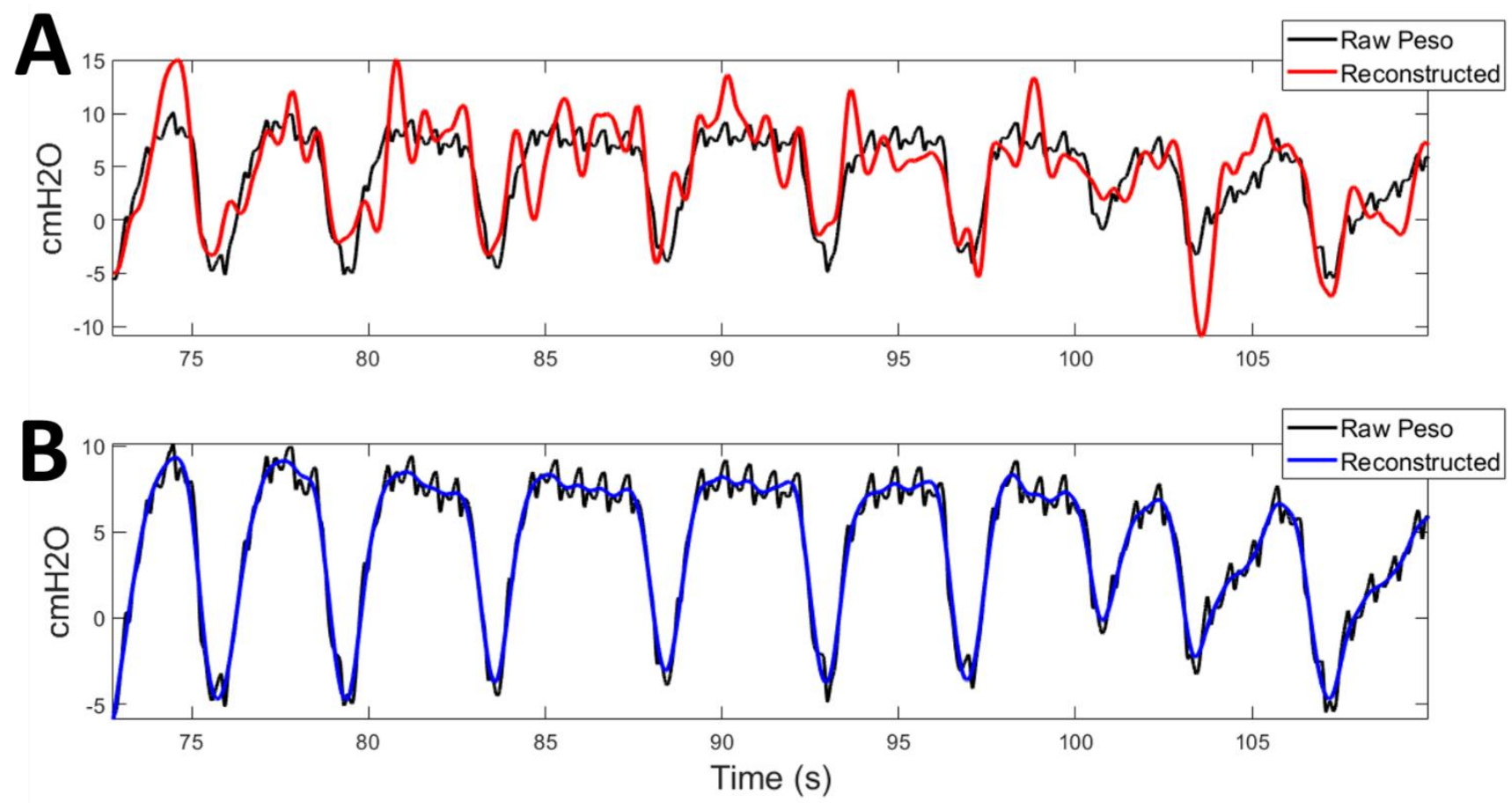

Figure 5.8. The Peso segment highlighted in green from Figure 5.8. The raw Peso is shown in black in both figures. (A) The reconstructed Peso signal is shown in red. The EEMD algorithm was applied to the raw signal with the esophageal spasms still in tact. (B) The reconstructed Peso signal shown in blue. The esophageal spasm from the raw signal was clipped manually before applying the EEMD algorithm.

$\underline{\text { Time Requirements }}$

One major limitation that is imposed by the EEMD algorithm is the amount of time required to decompose a signal. The proposed technique was applied to each of the 75 Peso signals in Dataset \#3 on a Precision T1700 Desktop Workstation running Windows 7 with an Intel® Xeon ${ }^{\circledR}$ E3-1225 v3 processor and 24 GB of RAM. The plot shown in Figure 5.9 demonstrates the time required to decompose a Peso signal using EEMD with respect to the duration of the Peso signal. The scatter plot and the corresponding curve fitting model indicates that there is a power law relation between the two aforementioned variables. As a result, longer duration signals would require a significant amount of time to decompose via EEMD. Other factors that can affect the proposed enhancement scheme are large transients, such as the esophageal spasms described in 
the previous section, as well as higher sampling rates, which increases the amount of datapoints. The amount of time required to run the proposed technique is important to consider for clinical applications; if time requirements are not a critical aspect of the task, then the proposed technique may be used. However, the proposed enhancement scheme in its current form may not be appropriate for applications such as real-time monitoring.

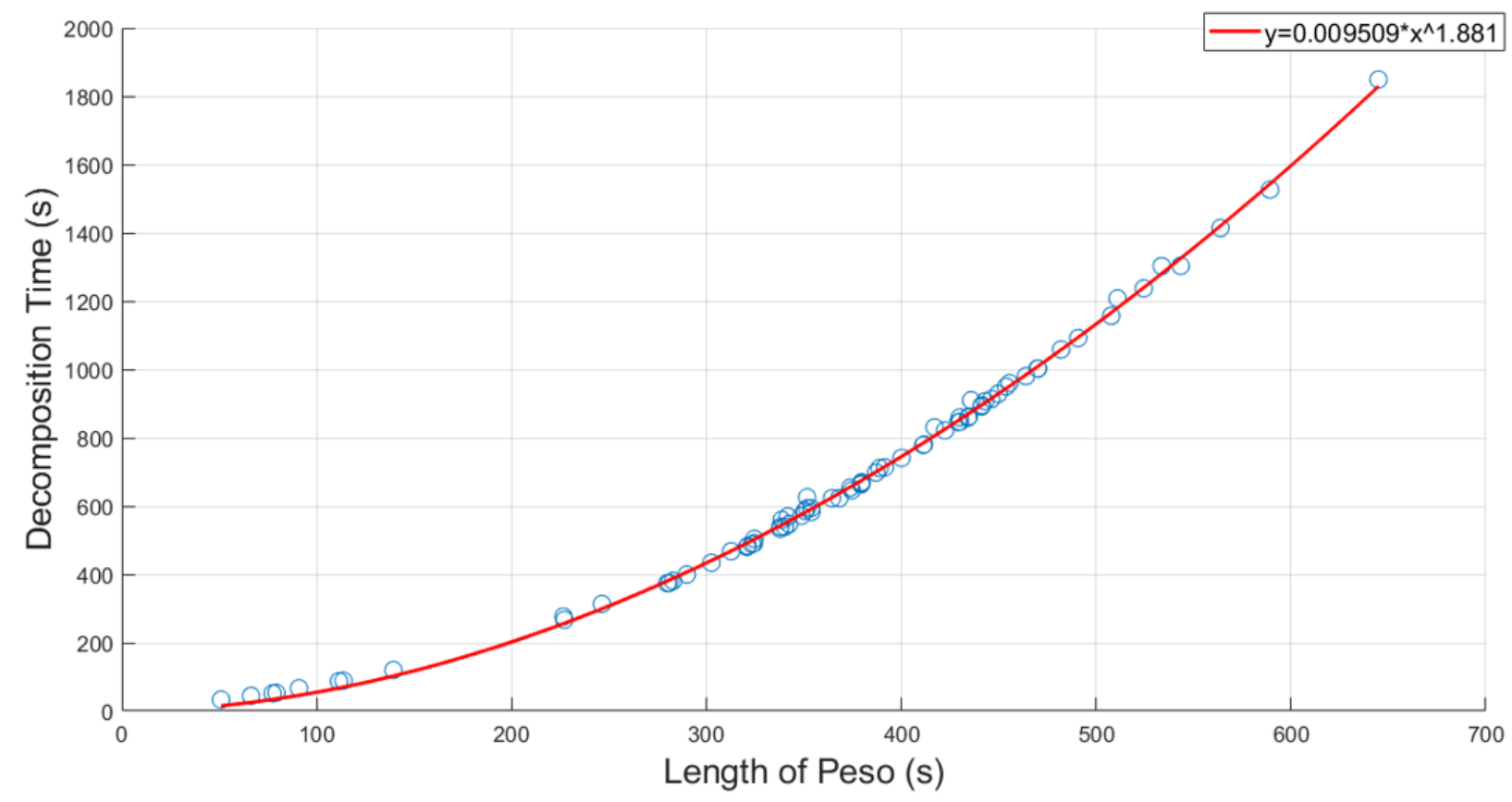

Figure 5.9. The relation between the amount of time required to decompose a Peso signal using EEMD with respect to the duration of the Peso signal.

\subsection{Chapter Summary}

This Chapter was dedicated to applying the proposed enhancement scheme, which has been developed through this thesis, to mechanically ventilated ICU patients. The dataset used in this Chapter helpful in testing the robustness of the technique, because the data was collected from 25 different patients. Thus, the data encompasses variability in the CGO signals both in terms of 
morphology as well as bandwidth. Additionally, the dataset is unique in the fact that each of the 25 patients were put under three different breathing modes. This enables us to test the technique on signals that capture both the inter-patient variability of the Peso waveforms, as well as intrapatient variability resulting from different modes of breathing. Face Validation was used as a means of testing the enhancement scheme's performance. The feedback from our collaborators showed that the technique was able to suppress much of the CGO. For many of the reconstructed Peso signals, there was a consensus that they may be used for further interpretation. However, it is still important to note that there are still concerns regarding the loss of information in the $\Delta \mathrm{P}$ amplitude. This Chapter concluded with a discussion of the limitations of the enhancement scheme, such as the fact that transients in the signal caused by esophageal spasms negatively affects the decomposition and subsequent reconstruction. As a result, esophageal spasms must be removed before applying the technique. Additionally, there are time constraints imposed by the EEMD algorithm, as it was shown that there is a power model relation between the amount of time required to decompose the Peso signal and the length of duration of the Peso signal. At present, the technique cannot be used in real-time applications, but it may be ideal for offline analysis of Peso signals. 


\section{Chapter 6}

\section{Conclusions and Future Work}

This thesis addressed the problem of CGO interference in Peso signals obtained from mechanically ventilated patients. From a biomedical signal processing perspective, suppressing CGOs from Peso signals is a challenging task, because both processes have bandwidths that can overlap with each other. Furthermore, inter-patient and intra-patient variabilities exist between both signals in terms of bandwidth. As a result, conventional filtering approaches may not be appropriate for signal quality enhancement. From a clinical perspective, enhancing the quality of Peso signals gives clinicians the ability to guide their mechanical ventilation strategies more effectively and empowers clinicians to personalize their patients' treatments. Ultimately, the aim is to reduce ICU stays and improve patient outcomes, which has societal and financial implications on the greater health care system and its associated funding agencies.

To conclude the work in this thesis, this Chapter will summarize the main contributions presented in Chapters 3 to 5. A discussion of future directions for this work and the potential of adapting the proposed technique to other mechanical ventilation applications will then be provided.

\subsection{Contributions}

The enhancement scheme proposed in this thesis makes use of adaptive, data-driven decomposition techniques, namely EEMD, to separate the Peso and CGO processes into their respective IMFs. Compared to conventional filtering techniques, which are constrained by the assumptions of linearity and stationarity of the incoming signals, techniques such as EEMD are 
ideal options for biomedical signal processing applications, because they can handle the non-linear and time-varying nature of physiological processes. Furthermore, the proposed technique can deal with inter-patient variability to generate a set of IMFs that are unique to each person. The utility of data-driven decomposition algorithms as outlined in this section has formed the basis of the contributions presented in this thesis.

\subsubsection{Characterization of Peso and CGO}

Preliminary experimentation using EMD on passively breathing patients showed that the CGO and Peso can be separated into their respective IMFs; CGOs are represented by the first few IMFs, while the Peso are present in the latter IMFs. However, the mode-mixing problem was observed, which suggested that a different algorithm, specifically EEMD, may be more appropriate to ensure that the IMFs retained their physical meanings. Further experiments using EEMD as the decomposition algorithm found that the proposed enhancement scheme is not only capable of reliably reconstructing clean Peso signals in passively breathing patients, but is also able to extract CGO. Thus, the technique may be used to monitor both the respiratory mechanics of the patient as well as the activity of their heart.

\subsubsection{Objective Performance Measures}

Validation of the proposed technique's performance is based purely on the subjective assessment of domain-experts. Although Face Validation by our collaborating clinicians is considered to be the "gold-standard" performance measure, their opinions can vary; some clinicians may be satisfied with the amount of filtering, while other clinicians voice concerns over the loss of information. To supplement the clinicians' assessments, an objective approach to validating the filtering performance has been proposed using FrankEinstein's Peso signals. By 
varying the levels of CGO interference in FrankEinstein's Peso signals, experiments were able to show that increasing the levels of CGO interference while keeping the amplitude level of the Peso constant resulted in larger reconstruction errors. Furthermore, the experiments also provided insights into the operating limits of the technique, which suggest that the technique can be used up until the CGO amplitude is $\sim 30 \%$ of $\Delta \mathrm{P}$ with the caveat that information loss may be greater than the clinical threshold of $10 \%$.

\subsubsection{Feasibility in the Clinical Context}

The proposed technique, which was refined through the course of this work, was applied to real-world mechanically ventilated ICU patients under three different conditions: i) passive breathing, ii) spontaneous breathing with PSV, and iii) spontaneous breathing with t-tube. According to the collaborating clinicians, the enhancement scheme can reliably suppress CGO in many of these signals regardless of the breathing condition, breathing profile/pattern, or CGO morphology. However, the technique does come with several limitations. First, clinicians voiced some concerns regarding the loss of information, specifically in $\Delta \mathrm{P}$, for some Peso signals. Secondly, esophageal spasms, which are often present in spontaneously breathing patients, negatively impacts the reconstruction. Finally, the EEMD algorithm requires a significant amount of time to decompose long-duration signals. Thus, in the clinical context, the technique should be used as a tool to supplement the decisions made by clinicians. Moreover, the technique in its current form cannot be used for real-time monitoring and ventilation management, and is instead better suited for offline applications. 


\subsection{Future Work}

\section{Overcoming Technical Limitations}

In Section 6.1.3, technical limitations were introduced in the application of the proposed technique. In its current form, the technique has only been applied to full-duration signals offline, which results in long decomposition times. To adapt the enhancement scheme for real-time applications, it will first be necessary to implement the EEMD algorithm in a way that would allow streaming data to be decomposed.

\section{Knowledge Translation}

While real-time enhancement is important for respiratory monitoring and mechanical ventilation management, the current enhancement scheme can be used in a clinical research setting for the purposes of understanding the respiratory mechanics of critically ill patients. To facilitate knowledge translation, a stand-alone executable file with a graphical user interface (GUI) may be developed so that clinical researchers can incorporate the technique in their standard workflow.

\section{Adapting to other Mechanical Ventilation and Respiratory Monitoring Applications}

While the Peso signal is used as a means of monitoring a patient's respiratory mechanics and controlling the level of mechanical ventilation, it is important to note that it is a pneumatic signal resulting from pressure changes in the thoracic cavity. Other modes of mechanical ventilation are being developed to capture information directly from the phrenic nerve, which serves as the neural pathway from the respiratory centres of the brain, to control the level of ventilatory support [5]. However, capturing information directly from the phrenic nerve is presently not technically feasible [5]. To circumvent the challenges of the aforementioned approach, a mode known as Neurally Adjusted Ventilatory Assist (NAVA) has been developed 
[5]. NAVA captures the electrical activity of the diaphragm (EAdi), which has been shown to be a valid measurement of a patient's neural respiratory drive, in order to control the level of ventilatory support [63]. Because the EAdi signal is a proxy for the respiratory drive, NAVA has consistently been shown to detect and overcome the challenges associated with asynchrony, especially in populations where significant patient-ventilator asynchronies (e.g. neonates, COPD patients) are observed [64]-[69]. Thus, the EAdi is undoubtedly a promising control signal for mechanical ventilation management. However, just like the Peso signal, the EAdi signal is also contaminated with interference from the heart but in the form of the ECG [70]. In this case, the problem formulation is the same as the one presented in this thesis: activity from the cardiovascular system is the major source of contamination during respiratory system monitoring, which suggests that the enhancement scheme in this thesis may be adapted to suppress ECG from the EAdi.

The abovementioned challenge of suppressing ECG from EAdi is in fact one of the works that inspired this thesis. Although ECG suppression from EAdi is not currently an active research project, we are in contact with the inventors of the NAVA technology through the Institute of Biomedical Engineering, Science and Technology (iBEST), which is a collaboration between Ryerson University and St. Michael's Hospital in Toronto, Ontario, Canada. Through iBEST, we have gained access to a dataset of EAdi signals obtained from 13 infants and 15 adults receiving NAVA support during mechanical ventilation. The data is sampled at $2 \mathrm{KHz}$ and the duration of signals range between 3.6 minutes to 2.18 hours. As mentioned previously, the ECG signal is a major source of contamination in the EAdi, which can be attributed to the data acquisition setup shown in Figure 6.1. Similar to Peso acquisition, a catheter outfitted with electronic sensors is inserted into the esophagus and guided towards the diaphragm. Due to the anatomical positioning 
of the heart with respect to the diaphragm, the ECG signal is captured by the electrodes during EAdi acquisition.

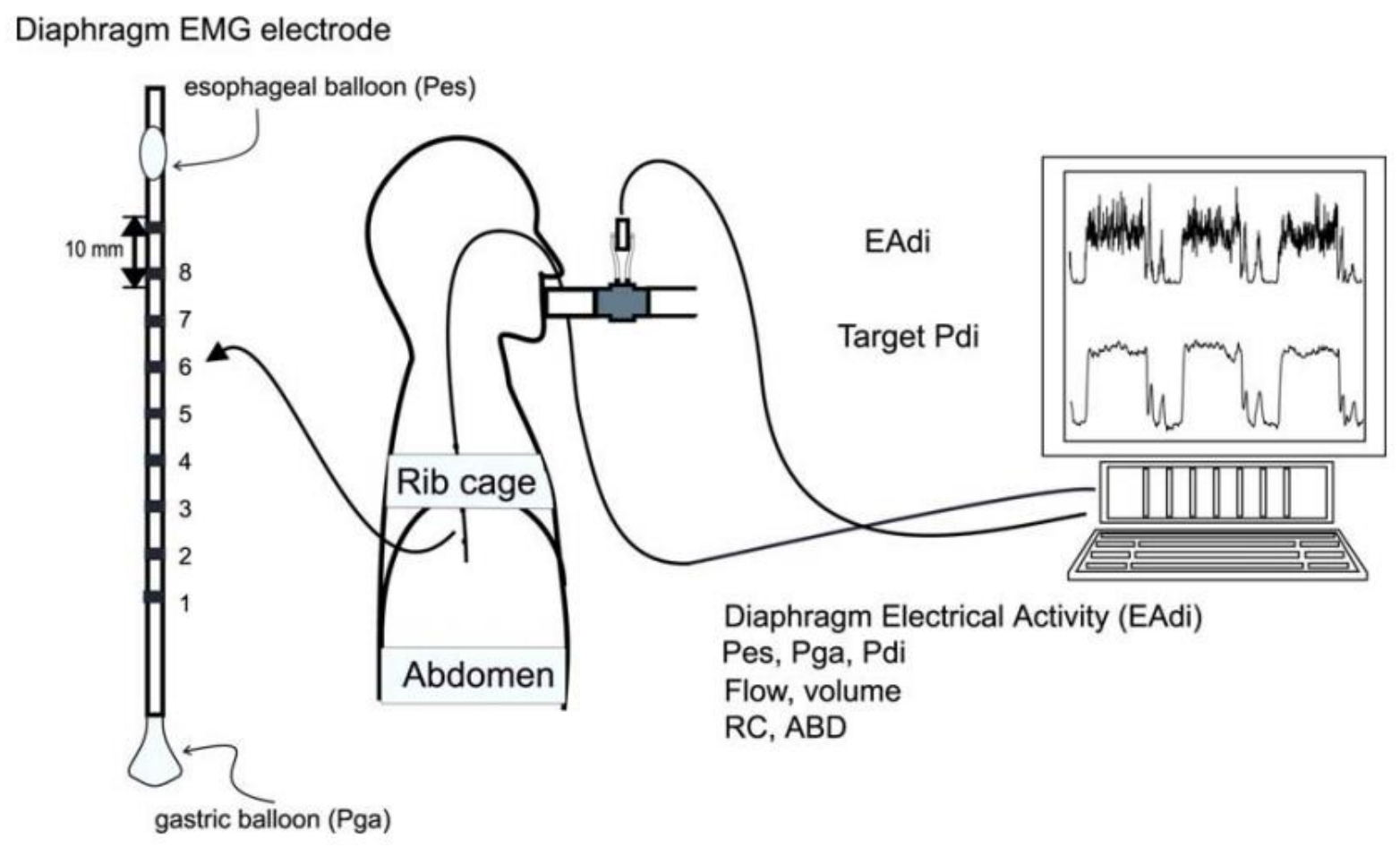

Figure 6.1. Experimental setup and data acquisition of EAdi signals. Source: [71].

The proposed enhancement scheme presented in this thesis using the EMD algorithm has been applied to the EAdi signals. Unfortunately, the preliminary results showed poor ECG suppression performance due to the mode-mixing problem. Furthermore, the technique was unable to decompose the EAdi signals in a reasonable timeframe due the high sampling rate, the large transients caused by the QRS complex, and the long duration of each signal. Thus, EMD and its variants may not be a suitable for this application. Based on the aforementioned limitations, future work related to ECG suppression in EAdi should ensure that the techniques are able to deal with the overlapping frequency spectra of both signals, as well as limiting the complexity of the algorithms such that they can converge in a reasonable amount of time. 


\section{References}

[1] Canadian Institute for Health Information, Care in Canadian ICUs. Ottawa, ON: CIHI, 2016.

[Online]. Available: https://secure.cihi.ca/free_products/ICU_Report_EN.pdf. [Accessed 26-Jul2019.

[2] Statistics Canada, 'Leading causes of death, total population, by age group', 2019. [Online]. Available: https://www150.statcan.gc.ca/t1/tbl1/en/tv.action?pid=1310039401. [Accessed 26Jul- 2019].

[3] American Thoracic Society, "Mechanical Ventilation," Am J Resp Crit Care Med, vol. 196, no. 2, pp. 3-4, 2017.

[4] J.D. Tobias, “Conventional mechanical ventilation,” Saudi J Anaesth, vol. 4, no. 2, pp. 86-98, 2010.

[5] C. Sinderby, P. Navalesi, J. Beck, Y. Skrobik, N. Comtois, S. Friberg, S.B. Gottfried and L. Lindstrom, "Neural control of mechanical ventilation in respiratory failure," Nature Medicine, vol. 5, no. 12, pp. 1433-1436, 1999.

[6] L. Blanch, A. Villagra, B. Sales, J. Montanya, U. Lucangelo, M. Luján, O. García-Esquirol, E. Chacón, A. Estruga, J. Oliva, A. Hernández-Abadia, G. Albaiceta, E. Fernández-Mondejar, R. Fernández, J. Lopez-Aguilar, J. Villar, G. Murias and R. Kacmarek, "Asynchronies during mechanical ventilation are associated with mortality," Intensive Care Med, vol. 41, no. 4, pp. 633$641,2015$.

[7] D. Dreyfuss and G.Saumon, "Ventilator-induced Lung Injury: Lessons from Experimental Studies," Am J Resp Crit Care Med, vol. 157, no. 1, pp. 294-323, 1996. 
[8] P.M. Reardon, S.M. Fernando, S.V. Katwyk, K. Thavorn, D. Kobewka, P. Tanuseputro, E. Rosenberg, C. Wan, B. Vanderspank-Wright, D. Kubelik, R.A. Devlin, C. Klinger and K. Kyeremanteng, "Characteristics, Outcomes, and Cost Patterns of High-Cost Patients in the Intensive Care Unit,” Critical Care Research and Practice, vol. 2018, pp. 1-7, 2018.

[9] Government of Canada, 'Canada's health care system', 2016. [Online]. Available: https://www.canada.ca/en/health-canada/services/canada-health-care-system.html. [Accessed 25Jul- 2019].

[10] Government of Ontario, 2019 Ontario Budget. 2019. [Online]. Available: http://budget.ontario.ca/pdf/2019/2019-ontario-budget-en.pdf. [Accessed 25- Jul- 2019].

[11] M. de Wit, K.B. Miller, D.A. Green, H.E. Ostman, C. Gennings and S.K. Epstein SK, "Ineffective triggering predicts increased duration of mechanical ventilation," Crit Care Med, vol. 37, no. 10, pp. 2740-2745, 2009.

[12] A.W. Thille, P. Rodriguez, B. Cabello, F. Lellouche and L. Brochard, "Patient-ventilator asynchrony during assisted mechanical ventilation," Intensive Care Med, vol. 32, no. 10, pp. 1515$1522,2006$.

[13] M.B.P. Amato, C.S.V. Barbas, D.M. Medeiros, R.B. Magaldi, G.P. Schettino, G. LorenziFilho, R.A. Kairalla, D. Deheinzelin, C. Munoz, R. Oliveira, T.Y. Takagaki and C.arlos R.oberto R. Carvalho, "Effect of a Protective-Ventilation Strategy on Mortality in the Acute Respiratory Distress Syndrome,” N Engl J Med, vol. 338, no. 6, pp. 347-354, 1998.

[14] D. Talmor, T. Sarge, A. Malhotra, C.R. O'Donnell, R. Ritz, A. Lisbon, V. Novack and S.H. Loring, "Mechanical Ventilation Guided by Esophageal Pressure in Acute Lung Injury," N Engl J Med, vol. 359, no. 20, pp. 2095-2104, 2008. 
[15] R. Arbour, "Cardiogenic Oscillation and Ventilator Autotriggering in Brain-Dead Patients: A Case Series," Am J Crit Care, vol. 18, no. 5, pp. 496-488, 2008.

[16] G.M. Plens, C.C.A. Morais, M.A. Nakamura, P.N. Souza, M.B.P. Amato, M.R. Tucci and E.L.V. Costa, "Effect of Cardiogenic Oscillations on Trigger Delay During Pressure Support Ventilation,” Respiratory Care, vol. 63, no. 7, pp. 865-872, 2018.

[17] H. Imanaka, M. Nishimura, M. Takeuchi, W.R. Kimball, N. Yahagi and K. Kumon, "Autotriggering caused by cardiogenic oscillation during flow-triggered mechanical ventilation," Crit Care Med, vol. 28, no. 2, pp. 402-407, 2000.

[18] T. Mauri, T. Yoshida, G. Bellani, E.C. Goligher, G. Carteaux, N. Rittayamai, F.Mojoli, D. Chiumello, L. Piquilloud, S. Grasso, A. Jubran, F. Laghi, S. Magder, A. Pesenti, S. Loring, L. Gattinoni, D. Talmor, L. Blanch, M. Amato, L. Chen, L. Brochard and J. Mancebo, "Esophageal and transpulmonary pressure in the clinical setting: meaning, usefulness and perspectives," Intensive Care Med, vol. 42, no. 9, pp. 1360-1373, 2016.

[19] C.L. VanPutte, J.L. Regan and A.F. Russo. "Respiratory System” in Seeley's Anatomy \& Physiology, $10^{\text {th }}$ ed. New York: McGraw-Hill, 2014, pp. 811-857.

[20] Respiratory System. 2007 [Online]. Available:

https://commons.wikimedia.org/wiki/File:Respiratory_system_complete_en.svg [Accessed: 3Jul- 2019].

[21] The Lung Pleura. 2013 [Online]. Available:

https://commons.wikimedia.org/wiki/File:2313_The_Lung_Pleurea.jpg [Accessed: 3- Jul- 2019]. 
[22] J.R. Beitler, A. Malhotra and B.T. Thompson, "Ventilator-Induced Lung Injury," Clin Chest Med., vol. 37, no. 4, pp. 633-646, 2016.

[23] J.H.T. Bates and B.J. Smith, "Ventilator-induced lung injury and lung mechanics,” Ann Transl Med, vol. 6, no. 19, pp. 1-13, 2018.

[24] D.R. Hess, "Respiratory Mechanics in Mechanically Ventilated Patients," Resp Care, vol. 59, no. 11, pp. 1773-1794, 2014.

[25] C. Mietto, M.L.N.G. Malbrain and D. Chiumello, "Transpulmonary pressure monitoring during mechanical ventilation: a bench to bedside review," Anaesthesiol Intensive Ther, vol. 47, pp. 27-37, 2015.

[26] M. Umbrello and D. Chiumello, "Interpretation of the transpulmonary pressure in the critically ill patient," Ann Transl Med, vol. 6, no. 19, pp. 1-12, 2018.

[27] P. Pelosi, M. Cereda, G. Foti, M. Giacomini M and A. Pesenti, “Alterations of lung and chest wall mechanics in patients with acute lung injury: effects of positive end-expiratory pressure," Am J Respir Crit Care Med, vol. 152, no. 2, pp. 531-517, 1995.

[28] V.M. Ranieri, N. Brienza, S. Santostasi, F. Puntillo, L. Mascia, N. Vitale, R. Guiliani, V. Memeo, F. Bruno, T. Fiore, A. Brienza and A.S. Slutsky, "Impairment of lung and chest wall mechanics in patients with acute respiratory distress syndrome: role of abdominal distension," Am J Respir Crit Care Med, vol. 156, no. 4, pp. 1082-1091, 1997.

[29] D.L. Grieco, L. Chen and L. Brochard, "Transpulmonary pressure: importance and limits," Ann Transl Med, vol. 5, no. 14, pp. 1-10, 2017. 
[30] T. Yoshida, M.B.P. Amato, D.L. Grieco, L. Chen, C.A.A. Lima, R. Roldan, C.C.A. Morais, S. Gomes, E.L.V. Costa, P.F.F. Cardoso, E. Charbonney, J.M. Richard, L. Brochard and B.P. Kavanagh, "Esophageal Manometry and Regional Transpulmonary Pressure in Lung Injury,” Am J Respir Crit Care Med, vol. 197, no. 8, pp. 1018-1026, 2018.

[31] E. Akoumianki, S.M. Maggiore, F. Valenza, G. Bellani, A. Jubran, S.H. Loring, P. Pelosi, D. Talmor, S. Grasso, D. Chiumello, C. Guérin, N. Patroniti, V.M. Ranieri, L. Gattinoni, S. Nava, P. Terragni, A. Pesenti, M. Tobin, J. Mancebo and L. Brochard, "The application of esophageal pressure measurement in patients with respiratory failure," Am J Respir Crit Care Med., vol. 189, no. 5, pp. 520-531, 2014.

[32] S.H. Loring, G.P. Topulos and R.D. Hubmayr, "Transpulmonary Pressure: The Importance of Precise Definitions and Limiting Assumptions," Am J Respir Crit Care Med., vol. 194, no. 12, pp. 1452-1456, 2015.

[33] P.L. Silva and P.R.M. Rocco, "The basics of respiratory mechanics: ventilator-derived parameters," Ann Transl Med, vol. 6, no. 19, pp. 1-11, 2018.

[34] S. Sahetya and R. Brower, "The Promises and Problems of Transpulmonary Pressure Measurements in ARDS," Curr Opin Crit Care, vol. 22, no. 1, pp. 7-13, 2016.

[35] F. Mojoli, F. Torriglia, A. Orlando, I. Bianchi, E. Arisi and M. Pozzi, “Technical aspects of bedside respiratory monitoring of transpulmonary pressure," Ann Transl Med., vol. 6, no. 19, pp. $1-8,2018$.

[36] S. Parthasarathy, A. Jubran and M. J. Tobin, "Assessment of Neural Inspiratory Time in Ventilator-supported Patients," Am J Respir Crit Care Med., vol. 162, pp. 546-552. 
[37] H. de Vries, A. Jonkman, Z. Shi, A. Spoelstra-de Ma and L. Heunks, “Assessing breathing effort in mechanical ventilation: physiology and clinical implications," Ann Transl Med., vol. 6, no. 19, 2018.

[38] L. Brander and A.S. Slutsky, "Invasive Mechanical Ventilation," in Clinical Respiratory Medicine, $4^{\text {th }}$ ed. S.G. Spiro, G.A. Silvestri and A. Agusti, Eds. Philadelphia, PA: Saunders, 2012, ch. 32, pp. 406-430. Accessed on: Jun. 4, 2019. [Online]. Available: SciencDirect.

[39] J. Bourenne, S. Hraiech, A. Roch, M. Gainnier, L. Papazian and J. Forel, "Sedation and neuromuscular blocking agends in acute respiratory distress syndrome," Ann Transl Med, vol. 5, no. 14, 2017.

[40] K.A. Samuelson, S. Larsson, D. Lundberg and B. Fridlund, "Intensive care sedation of mechanically ventilated patients: a national Swedish survey," Intensive and Critical Care Nursing, vol. 19, no. 6, pp. 350-362, 2003.

[41] L. Papazia, J. Forel, A. Gacouin, C. Penot-Ragon, G. Perrin, A. Loundou, S. Jaber, J. Arnal, D. Perez, J. Seghboyan, J. Constantin, P. Courant, J. Lefrant, C. Guérin, G. Prat, S. Morange and A. Roch, "Neuromuscular Blockers in Early Acute Respiratory Distress Syndrome," N Engl J Med, vol. 363, pp. 1107-1116, 2010.

[42] Y.P. Cheng, H.D. Wu, G.J. Jan, and C.Y. Wang, "Removal of cardiac beat artifact in esophageal pressure measurement via a modified adaptive noise cancellation scheme," Ann Biomed Eng, vol. 29, no. 3, pp. 236-243, 2001.

[43] J. Graßhoff, E. Petersen, M. Eger, G. Bellani and P. Rostalski, "A template subtraction method for the removal of cardiogenic oscillations on esophageal pressure signals," 2017 39th Annual 
International Conference of the IEEE Engineering in Medicine and Biology Society (EMBC), Jeju, South Korea, 2017, pp. 2235-2238.

[44] T.F. Schuessler, C.A. Volta, P. Goldberg, S.B. Gottfried, R.E. Kearney and J.H.T. Bates, “An adaptive filter for the reduction of cardiogenic oscillation on esophageal pressure signals," in Proc. of 17th International Conference of the Engineering in Medicine and Biology Society (EMBS), 20-23 Sept. 1995, Montreal, Canada, pp. 885-886.

[45] T.F. Schuessler, S.B. Gottfried, P. Goldberg, R.E. Kearney, and J.H.T. Bates, “An adaptive filter to reduce cardiogenic oscillations on esophageal pressure signals," Ann Biomed Eng, vol. 26, no. 2 , pp. $260-267,1998$.

[46] V.P. Seppä, J. Hyttinen and J. Viik, “A method for suppressing cardiogenic oscillations in impedance pneumography,” Physiol Meas, vol. 32, no. 3,pp. 337-345, 2011.

[47] N.E. Huang, "The empirical mode decomposition and the Hilbert spectrum for nonlinear and non-stationary time series analysis," Proc. R. Soc. Lond. A, vol. 454, no. 1971, pp. 903-995, 1998.

[48] G. Rilling, P. Flandrin, and P. Goncalves, "On empirical mode decomposition and its algorithms," IEEE-EURASIP workshop on nonlinear signal and image processing. NSIP-03, Grado, Italy, 2003.

[49] R. Balocchi, D. Menicucci, E. Santarangelo, L. Sebastiani, A. Gemignani, B. Ghelarducci, and M. Varanini, "Deriving the respiratory sinus arrhythmia from the heartbeat time series using empirical mode decomposition," Chaos, Solitons and Fractals, vol. 20, no. 1, pp. 171-177, 2004.

[50] E. Abdulhay, P Guméry, J. Fontecave, and P. Baconnier, "Cardiogenic oscillations extraction in inductive plethysmography: Ensemble empirical mode decomposition," 2009 31st Annual 
International Conference of the IEEE Engineering in Medicine and Biology Society (EMBS), Minneapolis, 2009, pp. 2240-2243.

[51] S. Pal and D. Mitra, "Empirical mode decomposition based ECG enhancement and QRS detection," Computers in Biology and Medicine, vol. 42, no. 1, pp. 83-92, 2012.

[52] J.A. Urigüen and B. Garcia-Zapirain, "EEG artifact removal - state-of-the-art and guidelines," J Neural Eng, vol. 12, no. 3, 2015

[53] M. Shahbakhti, V. Khalili and G. Kamaee, "Removal of blink from EEG by Empirical Mode Decomposition (EMD)," The 5th 2012 Biomedical Engineering International Conference, Ubon Ratchathani, 2012, pp. 1-5.

[54] S. Liu, R. X. Gao, D. John, J. Staudenmayer and P. Freedson, “Tissue Artifact Removal from Respiratory Signals Based on Empirical Mode Decomposition," Ann Biomed Eng, vol. 41, no. 5, pp. 1003-1015, 2013.

[55] H. Tjandrasa, S. Djanali and F.X. Arunanto, "Feature extraction using combination of intrinsic mode functions and power spectrum for EEG signal classification," in 2016 9th Int. Congr. Image and Signal Processing, BioMedical Engineering and Informatics (CISP-BMEI), Datong, China, 2016, pp. 1498-1502

[56] H. Hadoush, M. Alafeef, E. Abdulhay, “Automated identification for autism severity level: EEG analysis using empirical mode decomposition and second order difference plot," Behavioural Brain Research, vol. 362, pp. 240-248, 2019.

[57] Z. Wu and N.E. Huang, "Ensemble Empirical Mode Decomposition: A noise-assisted data analysis method," Advances in Adaptive Data Analysis, vol. 1, no. 1, pp. 1-41, 2009. 
[58] J. Zhang, R. Yan, R.X. Gao, and Z. Feng, "Performance enhancement of ensemble empirical mode decomposition," Mechanical Systems and Signal Processing, vol. 24, no. 7, pp. 2104-2123, 2010.

[59] User's Manual ASL 5000, SW 3.5th ed. IngMar Medical., Pittsburgh, PA, 2015.

[60] American Heart Association. (2015). All About Heart Rate (Pulse). [online] Available at: https://www.heart.org/en/health-topics/high-blood-pressure/the-facts-about-high-bloodpressure/all-about-heart-rate-pulse [Accessed 10 Feb. 2019].

[61] A.H. Kadish, A.E. Buxton, H.L. Kennedy, K.P. Knight, J.W. Mason, C.D. Schuger, C.M. Tracy, A.W. Boone, M. Elnicki, J.W. Hirshfield, B.H. Lorell, G.P. Rodgers, C.M. Tracy, and H.H. Weitz, "ACC/AHA clinical competence statement on electrocardiography and ambulatory electrocardiography. A report of the ACC/AHA/ACP-ASIM Task Force on Clinical Competence (ACC/AHA Committee to Develop a Clinical Competence Statement on Electrocardiography and Ambulatory Electrocardiography)," J. Am. Coll. Cardiol., vol. 38, no. 7, pp. 2091-2100, 2001.

[62] Y. Zigel, A. Cohen, and A. Katz, "The Weighted Diagnostic Distortion (WDD) Measure for ECG Signal Compression,” IEEE Trans BME, vol. 47, no. 7, pp. 1422-1430, 2000.

[63] J. Beck, S.B. Gottfried, P. Navalesi, Y. Skrobik, N. Comtois, M. Rossini and C. Sinderby, "Electrical Activity of the Diaphragm during Pressure Support Ventilation in Acute Respiratory Failure," Am J Resp Crit Care Med, vol. 164, no. 3, pp. 419-424, 2001.

[64] J. Spahija, M. de Marchie, M. Albert, P. Bellemare, S. Delisle, J. Beck and Christer Sinderby, "Patient-ventilator interaction during pressure support ventilation and neurally adjusted ventilatory assist," Crit Care Med., vol. 38, no. 2, pp. 518-526, 2010. 
[65] J.C. Ferreira, F. Diniz-Silva, H.T. Moriya, A.M. Alencar, M.B.P. Amato and C.R.R. Carvalho, "Neurally Adjusted Ventilatory Assist (NAVA) or Pressure Support Ventilation (PSV) during spontaneous breathing trials in critically ill patients: a crossover trial," BMC Pulmonary Medicine, vol. 17, no. 139, pp. 1-9, 2017.

[66] W. Verbrugghe and P.G. Jorens, "Neurally Adjusted Ventilatory Assist: A Ventilation Tool or a Ventilation Toy?," Respiratory Care, vol. 56, no. 3, pp. 327-335, 2011.

[67] O. Lamouret, L. Crognier, F.V. Bounes, J. Conil, C. Dilasser, T. Raimondi, S. Ruiz, A. Rouget, C. Delmas, T. Seguin, V. Minville and B. Georges, "Neurally adjusted ventilatory assist (NAVA) versus pressure support ventilation: patient-ventilator interaction during invasive ventilation delivered by tracheostomy," Crit Care, vol. 23, no. 2, pp. 1-9, 2019.

[68] F.C. Jalde, A.R. Almadhoob, J. Beck, A.S. Slutsky, M.S. Dunn and C. Sinderby, "Neurally Adjusted Ventilatory Assist and Pressure Support Ventilation in Small Species and the Impact of Instrumental Dead Space,” Neonatology, vol. 97, no. 3, pp. 279-285, 2010.

[69] J. Beck, M. Reilly, G. Grasselli, L. Mirabella, A.S. Slutsky, M.S. Dunn and C. Sinderby, "Patient-ventilator Interaction during Neurally Adjusted Ventilatory Assist in Very Low Birth Weight Infants," Pediatr Res, vol. 65, no. 6, pp. 663-668, 2009.

[70] C.A. Sinderby, J.C. Beck, L.H. Lindstrom and A.E. Grassino, "Enhancement of signal quality in esophageal recordings of diaphragm EMG," J. Appl. Physiol., vol. 82, no. 4, pp. 1370-1377, 1997.

[71] J. Spahija, J. Beck, L. Lindstrom, P. Begin, M. de Marchie and C. Sinderby, "Effect of increased diaphragm activation on diaphragm power spectrum center frequency," Respiratory Physiology \& Neurobiology, vol. 146, no. 1, pp. 67-76, 2005. 